\title{
NONPERTURBATIVE DYNAMICS OF STRONG INTERACTIONS FROM GAUGE/GRAVITY DUALITY
}

\author{
A Dissertation \\ Submitted to the Graduate Faculty of the \\ Louisiana State University and \\ Agricultural and Mechanical College \\ in partial fulfillment of the \\ requirements for the degree of \\ Doctor of Philosophy \\ in
}

The Department of Physics and Astronomy

by

Hovhannes Roman Grigoryan

B.Sc., Yerevan State University, 2001

M.Sc., Yerevan State University, 2003

August, 2008 


\section{Dedication}

To my parents Roman Grigoryan and Susanna Mamunts.

To my wife Anna Manukyan. 


\section{Acknowledgments}

I thank my adviser Prof. Jerry P. Draayer, for inviting me to start my doctoral degree at LSU, and presenting me with an opportunity to work at Jefferson Laboratory, which is one of the leading hadronic physics facilities in the world. Amongst many things, he always provided me with support to attend various important schools and conferences in theoretical physics, and collaborated with me on several science related projects. I also thank my JLab adviser Prof. Dr. Anthony $\mathrm{W}$. Thomas for his valuable advice during the course of my studies, for collaboration and for support to attend various scientific events. I am extremely grateful that he provided me with the opportunity to work in the directions of my interests.

I thank Prof. Anatoly V. Radyushkin for very fruitful collaboration, for various scientific conversations as well as for his advice and guidance. The work reported in this thesis was done in collaboration with him. I am grateful to Prof. José Goity for very interesting and inspiring conversations in the area of theoretical physics. I thank Professors Ian Balitsky and Rodney Crewther for very interesting and stimulating discussions. I also acknowledge Professors Robert Edwards, David Richards, Christian Weiss, Carl Carlson and Kostas Orginos for interesting science related conversations. I thank Professors Joshua Erlich and Christopher Carone for very pleasant collaborations, including very interesting and helpful discussions.

I also acknowledge Profs. George S. Pogosyan, Sergey I. Vinitsky and the late Dr. Vladimir V. Papoyan for supporting me at the Joint Institute of Nuclear Research in Dubna, Russia. I thank Wally Melnitchouk, Marc Sher, Alberto Accardi, Mark Paris for helpful advice regarding graduation, and Susan Brown for her assistance during my stay at JLab. Finally, I thank Kalin Drumev for providing me with the latex template for the thesis and helpful editorial suggestions.

My research was financially supported through the Department of Physics and Astronomy, Thomas Jefferson National Accelerator Facility, Southeastern Univer-

sities Research Association (SURA) and the Graduate School of Louisiana State University for which I am very grateful. Also, I thank Armenian Professional Society for the generous scholarship and SURA for the prestigious fellowship award. 


\section{Table of Contents}

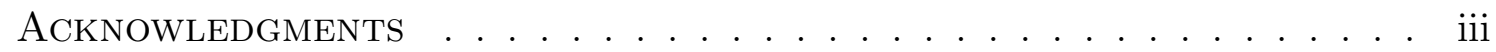

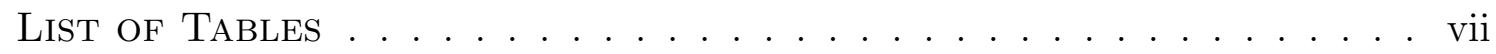

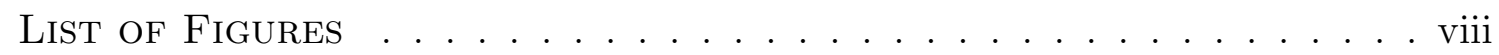

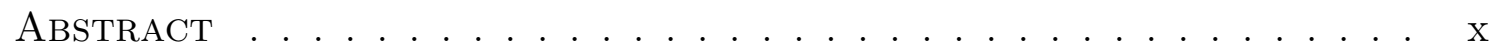

Chapter

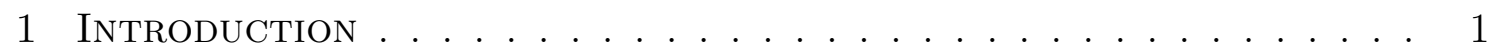

1.1 Historical Overview . . . . . . . . . . . . . . . . . . . 1

1.2 QCD and String Theory . . . . . . . . . . . . . . 2

1.3 Large $N_{c}$ QCD $\ldots \ldots \ldots \ldots \ldots \ldots \ldots \ldots$

1.4 D-branes . . . . . . . . . . . . . . . . . 5

1.5 AdS/QCD Model . . . . . . . . . . . . . . . . 6

2 Form Factors of Vector Mesons in Holographic QCD . . . . 9

2.1 Introduction . . . . . . . . . . . . . . . . . 9

2.2 Two-Point Function . . . . . . . . . . . . . . . . . . . . . 10

2.3 Three-Point Function . . . . . . . . . . . . . . . . . . . . . . . . . 12

2.4 Wave Functions . . . . . . . . . . . . . . . . . . . 15

2.5 Form Factors $\ldots \ldots \ldots \ldots \ldots$

2.6 Low- $Q^{2}$ Behavior . . . . . . . . . . . . . . . . . . . . . . . . . . . 19

2.7 Vector Meson Dominance Patterns _. . . . . . . . . . . . . 21

2.8 Large- $Q^{2}$ Behavior . . . . . . . . . . . . . . . . . . . . 22

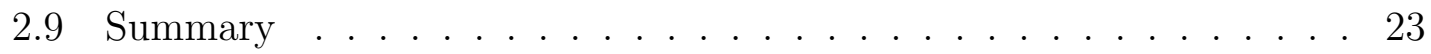

3 Form Factors in Holographic Model with Linear Confinement 25

3.1 Introduction . . . . . . . . . . . . . . . . 25

3.2 Preliminaries . . . . . . . . . . . . . . . . . . . 26

3.3 Bulk-to-Boundary Propagator $\ldots \ldots \ldots \ldots \ldots \ldots$

3.4 Three-Point Function . . . . . . . . . . . . . . . . . . 30

3.5 Form Factors . . . . . . . . . . . . . . . . . . . . . 31

3.6 Comparison with Hard-Wall Model . . . . . . . . . . . . . 35 
3.7 Summary $\ldots \ldots \ldots \ldots \ldots \ldots \ldots \ldots \ldots \ldots$

4 Massless Pion in Holographic Model of QCD . . . . . . . . . 39

4.1 Introduction . . . . . . . . . . . . . . . . . . . . 39

4.2 Preliminaries . . . . . . . . . . . . . . . . . . 40

4.2.1 Action and Equations of Motion . . . . . . . . . . . . 40

4.2 .2 Two-Point Function . . . . . . . . . . . . . . . . . . 42

4.2 .3 Pion Wave Functions . . . . . . . . . . . . . . . . . . . . 42

4.3 Pion Electromagnetic Form Factor . . . . . . . . . . . . . . . 43

4.3 .1 Three-Point Function . . . . . . . . . . . . . . . . . . . 43

4.3.2 Trilinear Terms in $F^{2}$ Part of Action . . . . . . . . . . . 44

4.3.3 Dynamic Factor and Wave Functions . . . . . . . . . . . . . 45

4.4 Wave Functions and Form Factor . . . . . . . . . . . . 47

4.4.1 Structure of Pion Wave Functions . . . . . . . . . . . . 47

4.4 .2 Pion Charge Radius . . . . . . . . . . . . . . . . . . . . . . . 49

4.4 .3 Form Factor at Large $Q^{2} \ldots \ldots \ldots \ldots$

4.5 Summary . . . . . . . . . . . . . . . 61

5 Anomalous Form Factor of Pion in AdS/QCD Model . . . . . . 63

5.1 Introduction . . . . . . . . . . . . . . . . . 63

5.2 Overview. . . . . . . . . . . . . . . . . 65

5.2 .1 AdS/QCD Action . . . . . . . . . . . . . . . 65

5.2 .2 Vector Channel . . . . . . . . . . . . . . . . . . . . 66

5.2 .3 Pion Channel . . . . . . . . . . . . . . . . . . . . . . . . 67

5.3 Anomalous Amplitude . . . . . . . . . . . . . . . . 68

5.3 .1 Isosinglet Fields . . . . . . . . . . . . . . . . . . . . . . 68

5.3 .2 Chern-Simons Term . . . . . . . . . . . . . . . . . 70

5.3 .3 Three-Point Function . . . . . . . . . . . . . . . . . . . . 72

5.3.4 Conforming to QCD Axial Anomaly . . . . . . . . . . . . 72

5.4 Momentum Dependence . . . . . . . . . . . . . . . . . 73

5.4 .1 Small Virtualities . . . . . . . . . . . . . . . . . . . . 73

5.4 .2 Large Virtualities . . . . . . . . . . . . . . . . . . . . . 74

5.5 Bound-State Decomposition . . . . . . . . . . . . . . 80

5.5 .1 One Real Photon . . . . . . . . . . . . . . . . . . 80

5.5.2 Two Deeply Virtual Photons . . . . . . . . . . . . . . . . . . 82

5.5.3 Structure of Two-Channel Pole Decomposition . . . . . . . . . 84

5.6 Summary . . . . . . . . . . . . . . . . . 86

6 Dimension Six Corrections to AdS/QCD Model . . . . . . . . . 88

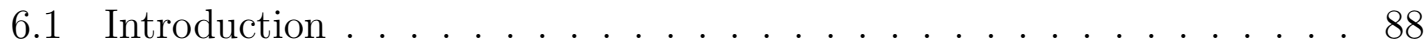

6.2 Preliminaries . . . . . . . . . . . . . . . . . . . . . . 89

6.3 The Effects From the $X^{2} F^{2}$ Term $\ldots \ldots \ldots \ldots$. . . . . . . 91

6.4 Corrections From the $F^{3}$ Term . . . . . . . . . . . . . 93

6.5 Form Factors . . . . . . . . . . . . . . . . . . . . . . 95

6.6 Results . . . . . . . . . . . . . . . . . . . 96 
6.7 Summary . . . . . . . . . . . . . . . . . 97

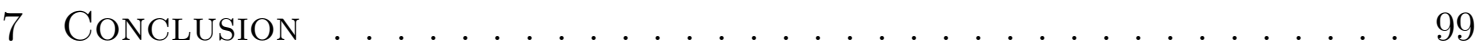

BIBLIOGRAPHY . . . . . . . . . . . . . . . . 109

Appendix: Permission Letters From Journals . . . . . . . . . . . 112

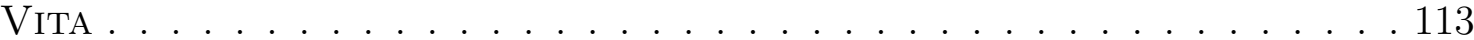




\section{List of Tables}

5.1 Coefficients $B_{n, k}$ in the hard-wall model. . . . . . . . . . . . . 85

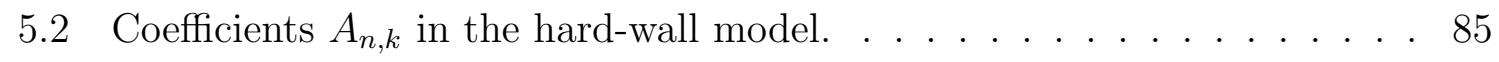

5.3 Coefficients $B_{n, k} M_{n}^{2} M_{k}^{2} / M_{1}^{4}$ in the hard-wall model. . . . . . . . . 86

5.4 Coefficients $A_{n, k} M_{n}^{2} M_{k}^{2} / M_{1}^{4}$ in the hard-wall model. . . . . . . . . 86 


\section{List of Figures}

1.1 Schematic picture of the AdS/QCD model. The vertical lines represent the slices of flat four dimensional Minkowski space-times. The horizontal line is the direction along the extra fifth dimension. QCD resides on UV brane which is a slice of Minkowski space at $z=0$. The confinement of QCD is generated by hand, cutting off the AdS space via the IR brane at $z=z_{0}$. According to holographic dictionary, the sources $A(x)$ of QCD operators, like $J(x)$, are promoted into a five dimensional theory to fully dynamical fields $A(x, z)$. The KK modes correspond to bound states of

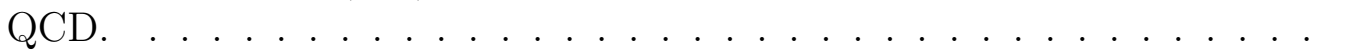

2.1 Schematic representation of the 3-gluon vertex. Vertical lines are the slices of flat four dimensional Minkowski spaces. Horizontal line is the direction along the extra fifth dimension. . . . . . . . . . . . . 13

2.2 Another schematic representation of the 3-gluon vertex. The disk at the bottom is the UV boundary of the AdS space, where the four dimensional QCD resides. The IR boundary is not shown in the picture. . . . . . . . 14

2.3 Eigenfunctions $\psi_{n}(z)$ as function of $z / z_{0}$ for first three modes. The number of modes of each curve determines $n \ldots \ldots \ldots \ldots$

2.4 Functions $z_{0} \phi_{n}(z)$ as function of $z / z_{0}$. The color on the curves corresponds to the same modes as in the previous plot. . . . . . . . 16

2.5 Plots of $F_{11}\left(Q^{2}\right)$ and $Q^{2} F_{11}\left(Q^{2}\right)$ as a function of $Q^{2}\left(\mathrm{GeV}^{2}\right) \ldots \ldots 18$

2.6 Plots of $G_{C}\left(Q^{2}\right)$ and $Q^{2} G_{C}\left(Q^{2}\right)$ as a function of $Q^{2}\left(\mathrm{GeV}^{2}\right) \ldots \ldots 20$

$3.1 Q^{2}$-multiplied $\rho$-meson form factor $\mathcal{F}_{00}\left(Q^{2}\right)$ (displayed in $\mathrm{GeV}^{2}$ ) as a function of $Q^{2}$ (given in $\mathrm{GeV}^{2}$ ) in hard-wall (upper line, red online) and soft-wall (lower line, blue online) models. . . . . . . . . . . . . . . 35 
4.1 Pion decay constant $f_{\pi}$ as a function of $a$ for fixed $\alpha^{1 / 3}=424 \mathrm{MeV}$. . . 49

4.2 Function $n(a) \ldots \ldots \ldots \ldots \ldots \ldots$

4.3 Functions $\varphi(\zeta, a)$ (top) and $\psi(\zeta, a)$ (bottom) for several values of $a: a=0$ (uppermost lines), $a=1, a=2.26, a=5, a=10$ (lowermost lines). . . . 51

4.4 Top: Function $\rho(\zeta, a)$ for $a=0, a=1, a=2.26, a=5, a=10$. Middle: Densities $\rho(\zeta, 2.26)$ for pion and $\rho_{\rho}(\zeta)$ for $\rho$-meson in the hard-wall model. Bottom: Same for densities multiplied by $\zeta \ldots$. . . . . . . . . . . . 52

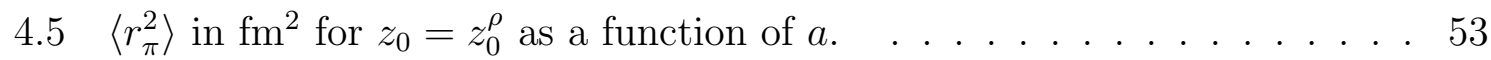

4.6 Top: Contributions to pion form factor $F_{\pi}\left(Q^{2}\right)$ from $\Psi^{2}$-term (lower curve), from $\Phi^{2}$-term (middle curve) and total contribution (upper curve). Bottom: Same for $Q^{2} F_{\pi}\left(Q^{2}\right) \ldots \ldots \ldots \ldots \ldots 5$

4.7 Top: Pion form factor $F_{\pi}\left(Q^{2}\right)$ from the holographic model (upper curve) in comparison with the monopole interpolation $F_{\pi}^{\text {mono }}\left(Q^{2}\right)$ (lower curve). Bottom: Ratio $F_{\pi}\left(Q^{2}\right) / F_{\pi}^{\text {mono }}\left(Q^{2}\right)$. . . . . . . . . . . . 57

4.8 Top: Model density $z \rho^{\bmod }(z)$ (measured in $\mathrm{fm}^{-1}$ ) is larger than the density $z\left|\Phi\left(z, \kappa_{\pi}\right)\right|^{2}$ for large $z$ (displayed in fm). Bottom: Ratio $F_{\pi}^{\bmod }\left(Q^{2}\right) / F_{\pi}^{\operatorname{mono}}\left(Q^{2}\right)$ for $B=1 / 4$.

4.9 Experimental results for the pion form factor $Q^{2} F_{\pi}\left(Q^{2}\right)$ compared with hard-wall holographic model and the model with soft-wall like ansatz (figure edited using the JLab resources). . . . . . . . . . . . . . . . . 60

5.1 Functions $\psi(\zeta, a)$ (top) and $\varphi(\zeta, a)$ (bottom) for several values of $a: a=0$ (uppermost lines), $a=1, a=2.26, a=5, a=10$ (lowermost lines).

5.2 Function $Q^{2} K\left(0, Q^{2}\right)$ in AdS/QCD model (solid curve, red online) and in local quark hadron duality model (coinciding with Brodsky-Lepage interpolation formula, dashed curve, blue online). The monopole fit of CLEO data is shown by dash-dotted curve (black online). . . . . . . . . 78

5.3 Form factor $K\left(0, Q^{2}\right)$ in AdS/QCD model (solid curve, red online) compared to BL interpolation formula (dashed curve, blue online). . . . . . 78

5.4 Form factor $K\left(Q^{2}, Q^{2}\right)$ in AdS/QCD model (solid curve, red online) compared to the local quark-hadron duality model prediction (dashed curve, blue online). 


\section{Abstract}

This thesis studies important dynamical observables of strong interactions such as form factors. It is known that Quantum Chromodynamics (QCD) is a theory which describes strong interactions. For large energies, one can apply perturbative techniques to solve some of the QCD problems. However, for low energies QCD enters into the nonperturbative regime, where different analytical or numerical tools have to be applied to solve problems of strong interactions. The holographic dual model of QCD is such an analytical tool that allows one to solve some nonperturbative QCD problems by translating them into a dual five-dimensional theory defined on some warped Anti de Sitter (AdS) background.

Working within the framework of the holographic dual model of QCD, we develop a formalism to calculate form factors and wave functions of vector mesons and pions. As a result, we provide predictions of the electric radius, the magnetic and quadrupole moments which can be directly verified in lattice calculations or even experimentally. To find the anomalous pion form factor, we propose an extension of the holographic model by including the Chern-Simons term required to reproduce the chiral anomaly of QCD. This allows us to find the slope of the form factor with one real and one slightly off-shell photon which appeared to be close to the experimental findings. We also analyze the limit of large virtualities (when the photon is far off-shell) and establish that predictions of the holographic model analytically coincide with those of perturbative QCD with asymptotic pion distribution amplitude. We also study the effects of higher dimensional terms in the AdS/QCD model and show that these terms improve the holographic description towards a more realistic scenario. We show this by calculating corrections to the vector meson form factors and corrections to the observables such as electric radii, magnetic and quadrupole moments. 


\section{Chapter 1}

\section{Introduction}

\subsection{Historical Overview}

The idea that the matter distributed all over the universe is composed of tiny inseparable building blocks, called atoms, has been with us for a very long time. However, it is only in the beginning of the last century, scientists such as Rutherford realized that the atom is composite, and consists of a tiny nucleus and electrons orbiting around it. The electrons that orbit near the outer layers of the atom are called valence electrons. These electrons determine the chemical properties of different elements classified in the periodic table. Later it was realized that nuclei themselves are made of protons and neutrons, collectively called nucleons. The number of protons determines the positive charge of the nuclei which places the atom in a particular place of the periodic table. The atoms which have the same number of protons and different amount of neutrons are called isotopes.

As opposed to electrons which are bound in the atom by electromagnetic forces, nucleons are held together by the so-called strong forces. All particles which interact via these strong forces are collectively called hadrons. Hadronic physics studies the properties of hadrons and the nature of strong forces among them. Hadrons are classified into mesons which have integer spins and baryons with half-integer spins. Examples of mesons are pion, kaon, rho and omega (which are denoted as $\pi, K$, $\rho$ and $\omega$, correspondingly). Examples of baryons are the nucleon and the delta (denoted as $N$ and $\Delta$ ). In the early 70's the large amount of experimental data provided clear evidence that the hadrons themselves consist of more elementary point-like particles named quarks and gluons that carry the so-called color charges which lie at the origin of strong forces.

The theory that studies the strong interactions in the language of quarks and gluons is called quantum chromodynamics (QCD). In nature, quarks and gluons can't be observed in separation since they are confined in the hadrons by the color forces. It also appears, that as the quarks move closer and closer together the magnitude of the color force between them decreases and the quarks behave as if they are almost free. This peculiar phenomenon is known as asymptotic freedom*. Understanding

\footnotetext{
*The pioneering papers can be found in [1].
} 
how quarks and gluons are distributed in the hadrons and how the hadrons interact together under various conditions (such as finite density and temperature) is essential when studying the properties of matter.

Various analytical and numerical methods have been developed to study QCD. One example is perturbative QCD which works at small distances where the coupling is weak, but fails to work at larger distances where the coupling becomes relatively strong in which case the problem is said to become nonperturbative. Examples of methods that study nonperturbative problems are effective field theories such as chiral perturbation theory, lattice QCD (see e.g. Ref. [2]), Dyson-Schwinger equations (DSE) formalism ${ }^{\dagger}$ and gauge/gravity duality ${ }^{\ddagger}$.

The four forces of nature: strong, electromagnetic, weak and gravitational are the origin of all known interactions in the universe. Among these forces, the weak interactions are responsible for the decay of neutrons and some hadrons in general. There is no evidence that there exists some additional force which is required to explain observations. Yet, it appears that even these four forces are not independent. Just as electric and magnetic forces were first considered to be independent and ultimately united by Maxwell into the electromagnetic force, the electromagnetic and weak forces were united into a single electroweak force by Glashow, Salam and Weinberg at the end of 1960's. Particle physics today is well-described by the so-called Standard Model which describes the electromagnetic and the weak interactions as two different aspects of a single electroweak interaction. In the Standard Model, all matter in the Universe consists of quarks and leptons interacting strongly via gluons, electromagnetically via photons, and weakly via $W^{ \pm}$and $Z$ bosons. Attempts to unify the electroweak and the strong interactions into a socalled Grand Unified Theory have also been made, however, to date there is no convincing experimental evidence that nature is described by this theory.

In all of these attempts to unify the forces of nature, gravity has always been treated separately. The challenge with gravity is that it can't be formulated in terms of a consistent quantum theory where all of the infinities can be removed by appropriate renormalization. Only at the end of the last century did the best possible candidate emerge which unified all forces into a framework called superstring theory $^{\S}$. Unfortunately, this theory only applies to short distances; so short, in fact, that it cannot currently be verified experimentally.

\subsection{QCD and String Theory}

Among the four fundamental forces of nature, strong interactions are of special interest. Many of the theoretical models known today trace back to attempts to understand the strong interaction. In particular, string theory originated from an attempt to describe a large proliferation of mesons and baryons that were experimentally observed in the '50's. It was suggested in the 1960's that all known hadrons

\footnotetext{
${ }^{\dagger}$ A good review on applications of DSE formalism can be found in Ref. [3].

†Pioneering works can be found in [4]. For an extended review, see Ref. [5].

${ }^{\S}$ Some of the textbooks on string theory can be found in Refs. $[6,7,8,9]$.
} 
were different oscillating modes of a single vibrating string. However, in the beginning of the 1970's it became clear that the hadronic physics must be described by QCD, and since string theory contained a massless spin-2 particle, which was known to be responsible for the gravitational force, string theorists shifted their attention to Planck scales, to find a theory of quantum gravity.

The excited states of hadrons are called resonances, which can be arranged into approximately linear Regge trajectories, determined by the relation $J=\alpha(s)$, where $J$ is the angular momentum and $s=M^{2}$. A resonance occurs for such $s$ for which $\alpha(s)$ is either positive integer (mesons) or positive half integer (baryons). The trajectory corresponding to the largest value of $J$ at a given $s$ is called leading trajectory. It was experimentally observed that these leading trajectories were almost linear; that is, $\alpha(s)=\alpha(0)+\alpha^{\prime}(0) s$, where $\alpha(0)$ is known as the Regge intercept which depends on various quantum numbers. However, observations showed that $\alpha^{\prime}$ which is known as the Regge slope, appeared to have a universal value $\left(\sim 1 \mathrm{GeV}^{2}\right)$.

This was precisely one of the motivations for hadronic string theory, the fact that hadrons can be arranged into approximately linear Regge trajectories:

$$
J=\alpha^{\prime} M^{2}+\alpha(0)
$$

where $M$ is the mass of the hadron and $J$ its angular momentum (spin). This feature of Regge trajectories can be derived from the simple assumption that the hadrons are described by rotating relativistic strings. Indeed, imagine a rigidly rotating string, the endpoints of which move at near the speed of light $c$. Let's assign a coordinate system along the string, such that $r \in[-L / 2, L / 2]$ is the coordinate variable and $L$ is the length of the string. Then, the linear velocity is $v(r)=\omega r$, where $\omega=c /(L / 2)$. Therefore, energy of the rigidly rotating string with constant tension $T$ (in units $c=1$ ) is

$$
E=T \int_{-L / 2}^{L / 2} \frac{d r}{\sqrt{1-v^{2}}}=T L \int_{0}^{1} \frac{d x}{\sqrt{1-x^{2}}}=\frac{\pi}{2} T L,
$$

and the angular momentum is

$$
J=T \int_{-L / 2}^{L / 2} \frac{v r d r}{\sqrt{1-v^{2}}}=\frac{1}{2} T L^{2} \int_{0}^{1} \frac{x^{2} d x}{\sqrt{1-x^{2}}}=\frac{\pi}{8} T L^{2} .
$$

Now, since $E=M$, one can deduce that $J=\alpha^{\prime} M^{2}$, where $\alpha^{\prime} \equiv 1 /(2 \pi T)$ and $\alpha(0)=0$. While these semi-classical calculations are certainly incomplete, they nonetheless grasp the essence of a string description of hadrons.

The other motivation came from the duality conjecture formulated by Dolen, Horn and Schmid [10], based on the studies of $\pi N$ scattering, stating that the sum over $s$-channel exchanges equals the sum over $t$-channel ones. These observations inspired Veneziano to propose, in Ref. [11], the analytic form of a manifestly dual 4-point amplitude of the form:

$$
A(s, t) \sim \frac{\Gamma[-\alpha(s)] \Gamma[-\alpha(t)]}{\Gamma[-\alpha(s)-\alpha(t)]} .
$$


This amplitude implies an exactly linear Regge trajectory $\alpha(s)=\alpha(0)+\alpha^{\prime} s$. Later it was shown by Nambu [12], Nielsen [13] and Susskind [14] that this amplitude can be obtained from interpreting the hadrons as vibrating strings (see also a short talk on the relation of string theory to QCD in Ref. [15]).

However, in spite of giving a very attractive visual picture of hadrons, string theory as a theory of strong interactions was abandoned because of fundamental inconsistencies. In particular, quantum consistency of the Veneziano's model required $\alpha(0)=1$, which implied that the lightest of the spin-1 states (the lightest vector meson) is massless, and the lightest of the spin-0 states is a tachyon (particle with negative mass squared). However, such hadrons don't exist in nature, since the lightest of the vector mesons, the $\rho$ meson, is not massless and there are simply no tachyons. Moreover, it appears that a bosonic string theory is only consistent in 26 space-time dimensions. Meanwhile, the so-called supersymmetric string theories are only valid in 10 dimensions. These problems, together with extra dimensions and supersymmetry, lost the connection of string theory with hadronic physics. However, string theory wasn't completely abandoned since it gave hope for describing something else; namely, since the graviton appears naturally in the closed string spectrum as a massless spin-2 particle, string theory gave hope for a theory of quantum gravity and more, unifying gravity with the other known forces of nature.

Years later, string theory found a path back into hadronic physics due to peculiar features of QCD. It appears that at short distances $(\ll 1 \mathrm{fm})$, the quark anti-quark potential is Coulombic due to asymptotic freedom. However, at large distances the potential is linear due to the formation of a confining flux tube between quarks. In other words, if we try to separate a quark from the anti-quark, a flux tube will be formed between them. To understand this, notice, that if $q$ is a quark field, then the operator $\bar{q}(0) q(x)$ is not gauge invariant. However, if we add an additional object $W(x)$ between the quark fields, where

$$
W(x)=P \exp \left(i \int_{0}^{x} A_{\mu} d x^{\mu}\right),
$$

is called the Wilson line, then the modified operator $\bar{q}(0) W(x) q(x)$ will be gauge invariant. This Wilson line can be thought of as a flux tube which extends between the quark and anti-quark.

It appears that, when these flux tubes are much longer than their thickness, it is possible to describe them by semi-classical Nambu strings, the quantization of which predicts a quark anti-quark potential [16] of the form

$$
V(r)=T r+\mu+\frac{\gamma}{r}+O\left(1 / r^{2}\right)
$$

where $\gamma=-\pi(d-2) / 24$. Recent lattice calculations [17] by Lüscher of the force versus distance for probing quarks and anti-quarks produce good agreement with this value in $d=3$ and $d=4$ for $r>0.7 \mathrm{fm}$. Based on this, one can conclude that 
long QCD strings are well described by the Nambu-Goto area action,

$$
S_{N G}=-T \int d \sigma d \tau \sqrt{-\operatorname{det} \partial_{a} X^{\mu} \partial_{b} X_{\mu}}
$$

where $a, b \in\{\sigma, \tau\}$ ( $\sigma$ and $\tau$ are the string world sheet coordinates).

\section{3 $\quad$ Large $N_{c}$ QCD}

QCD is a gauge theory based on the group $S U(3)$. As a result, it is sometimes said that quarks in QCD carry 3 colors. In the beginning of the 1970's, 't Hooft suggested [18] that the theory might simplify when the number of colors $N_{c}$ is taken to be large. In this case, the expansion parameter would be $1 / N_{c}$, and the hope is that one could solve QCD exactly for $N_{c} \rightarrow \infty$, then perform an expansion in $1 / N_{c}=1 / 3$. To make the large $N_{c}$ limit meaningful, one should keep the so-called 't Hooft coupling $\lambda=g_{\mathrm{YM}}^{2} N_{c}$ fixed.

This generalization of QCD from 3 colors to $N_{c}$, strengthened the connection between gauge theory and string theory. To understand why, notice that in the large $N_{c}$ limit, all of the Feynman graphs can be classified according to the topological Euler characteristic of the graph. Therefore, summing the graphs with a given topology is equivalent to summing over the world sheets of some sort of string ? It appears that in the large $N_{c}$ limit the gauge theory significantly simplifies, since only the planar diagrams contribute.

\subsection{D-branes}

String theory contains an important object called a Dirichlet $p$-brane (or $D p$-brane for short) which is a $p+1$ dimensional hyperplane in $9+1$ dimensional spacetime where strings are allowed to end (for pioneering paper, see Ref. [19]). The end-points of these strings in the $p+1$ longitudinal coordinates (where the $D p$ brane lies) satisfy the so-called free Neumann boundary conditions, while the $9-p$ coordinates transverse to the $D p$-brane have the so-called fixed Dirichlet boundary conditions (this is why this object is called a "Dirichlet brane").

The most important property of D-branes is that they contain gauge theories on their world volume. In particular, the massless spectrum of open strings living on a $D p$-brane contains a (maximally supersymmetric) $U(1)$ gauge theory in $p+1$ dimensions. Moreover, it appears that if we consider the stack of $N$ coincident $D$-branes, then there are $N^{2}$ different species of open strings which can begin and end on any of the $D$-branes, allowing us to have the (maximally supersymmetric) $U(N)$ gauge theory on the world-volume of these $D$-branes. Now, if $N$ is sufficiently large, then this stack of $D$-branes is a heavy object embedded into a theory of closed strings that contains gravity. This heavy object curves the space which can then be described by some classical metric and other background fields.

『For a particular realization of this idea, see Refs. [20, 21] 
Thus, we have two absolutely different descriptions of the stack of coincident $D p$-branes. One description is in terms of the $U(N)$ supersymmetric gauge theory on the world volume of the $D p$-branes, and the other is in terms of the classical theory in some gravitational background. It is this idea that lies at the basis of gauge/gravity duality.

\subsection{AdS/QCD Model}

The AdS/CFT correspondence (or duality) [4] conjectures equivalence of gravity theory on the Anti de Sitter space $\mathrm{AdS}_{5}$ and a strongly coupled four-dimensional (4D) conformal field theory (CFT). This duality states that for every CFT operator $\mathcal{O}(x)$ there exists a corresponding bulk field $\Phi(x, z)$ that is uniquely determined by the boundary condition (b.c.) $\Phi(x, z=0)$ at the ultraviolet (UV) $4 \mathrm{D}$ boundary of AdS space ( $x$ denotes the $4 \mathrm{D}$ coordinates and $z$ stands for the fifth extra dimension). In particular, if $S_{5}\left[\phi_{0}(x)\right]$ is the gravity or string action of $\phi(x, z)$ with $\phi(x, 0)=$ $\phi_{0}(x)$, then the correspondence takes the form

$$
\left\langle\exp \left(i \int d^{4} x \phi_{0}(x) \mathcal{O}(x)\right)\right\rangle_{C F T}=\exp \left(i S_{5}\left[\phi_{0}(x)\right]\right) .
$$

For small $z$, the solution of the equations of motion is:

$$
\phi(x, z) \sim z^{4-\Delta} \phi_{0}(x)+\frac{1}{2 \Delta-4} z^{\Delta}\langle\mathcal{O}(x)\rangle,
$$

where $\Delta$ is a conformal dimension of quantum operator $\mathcal{O}(x)$, which has expectation value $\langle\mathcal{O}\rangle$, and $\phi_{0}$ is a normalizable mode, corresponding to a source of the quantum operator $\mathcal{O}$. The mass of the bulk field $\phi$ is given by $m_{\phi}^{2}=\Delta(\Delta-4)$.

The situation becomes more clear with the addition of the infrared (IR) brane, which corresponds to some deformation of the CFT leading to a breakdown of conformal invariance in the IR. In this case, we have both particles and S-matrix elements, and the statement of the holographic equivalence between the broken CFT and the gravitational picture is not only expressed in the abstract form but also allows one to explicitly check if the two theories have identical spectra and identical S-matrix elements. In particular, the KK gravitons in the gravity side can be interpreted in the $4 \mathrm{D}$ theory as resonances. It is also additionally conjectured that the AdS/CFT correspondence can be extended to tell us that any 5D gravity theory on $A d S_{5}$ is holographically dual to some strongly coupled, large $N_{c}, 4 \mathrm{D}$ gauge theory [22]. The task of holographic models of QCD is to find a gravity theory for which the dual theory is as close to QCD as possible. Different holographic models were proposed incorporating different aspects of QCD.

Holographic duals of QCD based on the AdS/CFT correspondence have been applied recently to hadronic physics (see, e.g., [23, 24, 25, 26, 27, 28, 29, 58, 30, 32, $33,34]$ ). These models are able to incorporate essential properties of QCD such as confinement and chiral symmetry breaking, and have demonstrated in many cases success in determination of static hadronic properties, such as resonance masses, 
decay constants, chiral coefficients, etc. Amongst the dual models, a special class is the so-called "bottom-up" approaches (see, e.g., [26, 27, 28, 32]), the goal of which is to reproduce known properties of QCD by choosing an appropriate theory in the 5-dimensional AdS bulk. Within the framework of the AdS/QCD models, by modifying the theory in the bulk one may try to explain/fit experimental results in different sectors of QCD.

Dynamic properties (form factors) were studied originally within the holographic approach of Ref. [23], and the connection between AdS/QCD approach of Refs. [23, $24]$ and the usual light-cone formalism for hadronic form factors was proposed in [30] and discussed in [35]. The calculation of form factors of scalar and vector hadrons within the approach of Ref. [23] was performed in [46], and applied to study the universality of the $\rho$-meson couplings to other hadrons. The expressions for hadronic form factors given in Refs. [23, 30, 46] have an expected form of $z$-integral containing the product of two hadronic wave functions and a function describing the probing current. However, the hadronic functions used in Ref. [30] strongly differ from those in Refs. [23, 46]. The latter give meson coupling constants through their derivatives at $z=0$ and satisfy Neumann b.c. at the IR boundary $z=z_{0}$, while the functions used in Ref. [30] satisfy Dirichlet b.c. at $z=z_{0}$, and are proportional (after extraction of the overall $z^{2}$ factor) to the meson coupling constants $f_{n}$ at the origin. In this respect they are analogous to the bound state wave functions in quantum mechanics, which makes their interpretation in terms of light-cone variables possible (as proposed in Ref. [30]).

Having offered the above as abbreviated background material to what follows, we now turn to the specific research focus of this thesis which is organized as follows. In Chapter 2, using the AdS/QCD model, a representation of the form factors in terms of generalized vector-meson dominance is derived, in which the form factors are saturated from the contributions of the first two bound vector-meson states. The electric radius of the rho-meson is shown to be in good agreement with predictions from lattice QCD. In Chapter 3, we use the holographic dual model of QCD with linear confinement behavior to develop a formalism for calculating hadronic observables. We show that for the rho-meson the basic elastic form factor exhibits perfect vector meson dominance. The electric radius of the rho-meson is calculated to be slightly larger than in the case of the hard-wall cutoff.

In Chapter 4, we study the pion in the chiral limit of QCD. We find an analytic expression for the pion decay constant in terms of two parameters of the model. We also find that the pion charge radius in the hard-wall model is smaller than the experimental value. In Chapter 5 , we study the anomalous form factor of the neutral pion in the framework of the holographic dual model of QCD with the Chern-Simons term. As a result, we calculate the slope of the form factor with one real and one slightly virtual photon and show that it is close to experimental findings. We also show that for large virtualities the predictions of the holographic model coincide analytically with those of perturbative QCD with asymptotic pion distribution amplitude.

In Chapter 6, we add dimension six terms into the vector sector of the AdS/QCD lagrangian and study their effect on the vector meson form factors. We show that the 


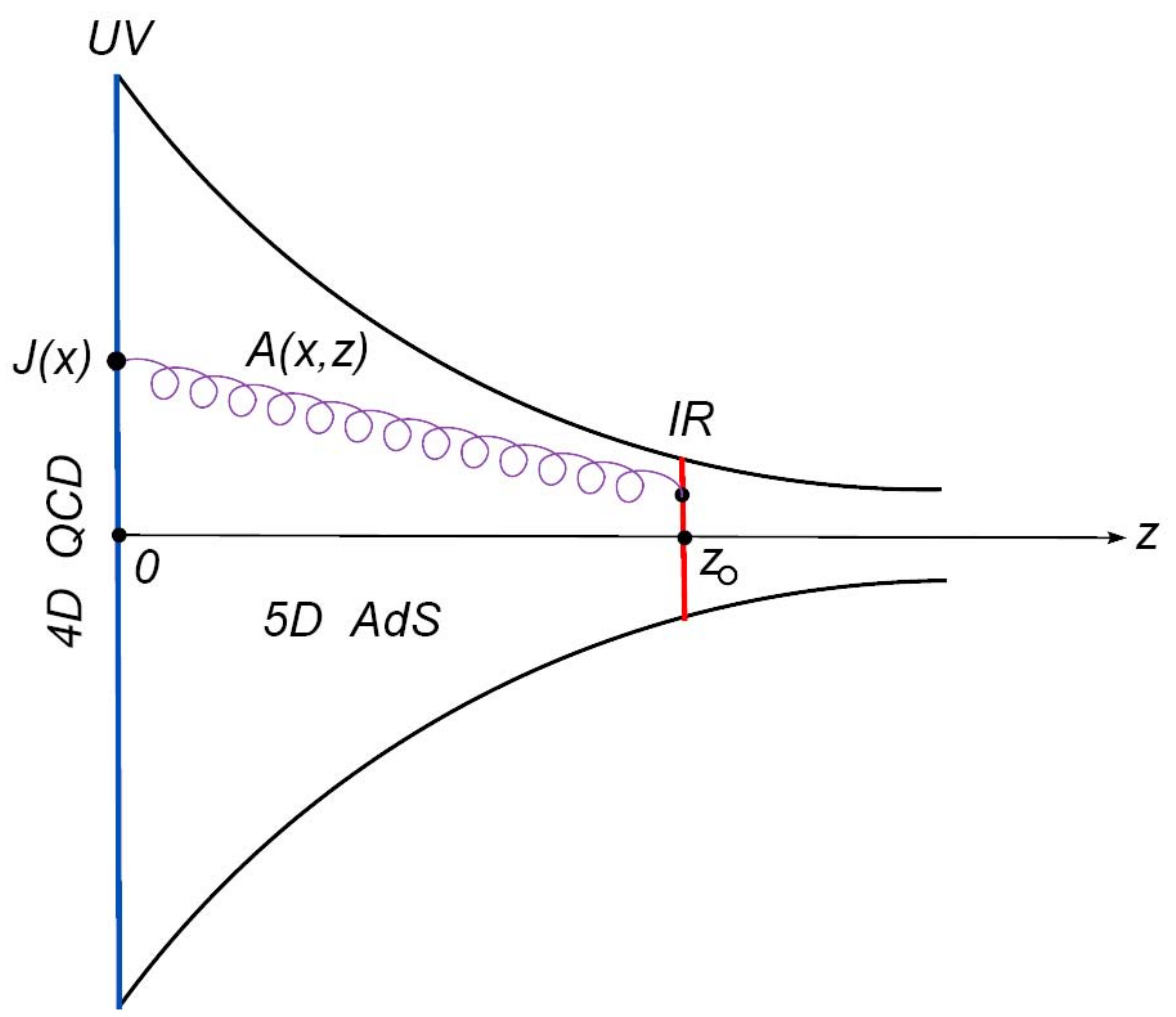

Figure 1.1: Schematic picture of the AdS/QCD model. The vertical lines represent the slices of flat four dimensional Minkowski space-times. The horizontal line is the direction along the extra fifth dimension. QCD resides on UV brane which is a slice of Minkowski space at $z=0$. The confinement of QCD is generated by hand, cutting off the AdS space via the IR brane at $z=z_{0}$. According to holographic dictionary, the sources $A(x)$ of QCD operators, like $J(x)$, are promoted into a five dimensional theory to fully dynamical fields $A(x, z)$. The KK modes correspond to bound states of QCD.

term, like $X^{2} F^{2}$, doesn't change the electric charge, the magnetic and the quadrupole moments, but affects the charge radius, the masses and the decay constants of the vector mesons. We also show that the term $F^{3}$ affects all the above mentioned observables and provides more realistic predictions for the AdS/QCD model. Finally, we summarize our results in the concluding chapter. 


\section{Chapter 2}

\section{Form Factors of Vector Mesons in Holographic QCD}

\section{$2.1 \quad$ Introduction}

In this chapter*, we study the form factors and wave functions of vector mesons within the framework of the holographic QCD model described in Refs. [27, 26, 28] (which will be referred to as the hard-wall model). To this end, we consider a 5D dual of the simplest $N_{f}=2$ version of QCD to be a Yang-Mills theory with the $S U(2)$ gauge group in the background of sliced AdS space, i.e., the $4 \mathrm{D}$ global $S U(2)$ isotopic symmetry of $N_{f}=2$ QCD is promoted to a $5 \mathrm{D}$ gauge symmetry in the bulk. Note, that the AdS/QCD correspondence does not refer explicitly to quark and gluon degrees of freedom. Rather, one deals with the bound states of QCD which appear as infinitely narrow resonances. The counterparts in the correspondence relation are the vector current $J_{\mu}^{a}(x)$ with conformal dimension $\Delta=3$ (in QCD, it may be visualized as $\left.\bar{q}(x) t^{a} \gamma_{\mu} q(x)\right)$, and the 5D gauge field $A_{\mu}^{a}(x, z)$.

We start with recalling the basic elements of the analysis of two-point functions $\langle J J\rangle$ given in Refs. [26, 27], and introduce a convenient representation for the $A$-field bulk-to-boundary propagator $\mathcal{V}(p, z)$ based on the Kneser-Sommerfeld formula [41] that gives $\mathcal{V}(p, z)$ as an expansion over bound state poles with the $z$-dependence of each pole contribution given by " $\psi$ wave functions", that are eigenfunctions of the 5D equation of motion with Neumann b.c. at the IR boundary. Then we study the three-point function $\langle J J J\rangle$ and obtain an expression for transition form factors that involves $\psi$ wave functions and the nonnormalizable mode factor $\mathcal{J}(Q, z)$. We write the latter as a sum over all bound states in the channel of electromagnetic current, which gives an analogue of the generalized vector meson dominance (VMD) representation for hadronic form factors. As the next step, we introduce " $\phi$ wave functions" that strongly resemble wave functions of bound states in quantum mechanics (they satisfy Dirichlet b.c. at $z=z_{0}$, and their values at $z=0$ give bound state couplings $g_{5} f_{n} / M_{n}$, i.e., they have the properties necessary for the light-cone interpretation of AdS/QCD results proposed in Ref. [30]). We rewrite form factors

\footnotetext{
*The main results from the Ref. [36] are printed by permission from the Elsevier, see Appendix.
} 
in terms of $\phi$ functions, formulate predictions for $\rho$-meson form factors, and analyze these predictions in the regions of small and large $Q^{2}$.

The $\rho$-meson electric radius is calculated, and it is also shown that hard-wall model predicts a peculiar VMD pattern when two (rather than just one) lowest bound states in the $Q^{2}$-channel play the dominant role while contributions from higher states can be neglected. This double-resonance dominance is established both for the $\rho$-meson form factor $F\left(Q^{2}\right)$ given by the overlap of the $\psi$-wave function (here we confirm the results obtained in Ref. [46] for the $\rho$-meson form factor considered there) and for the form factor $\mathcal{F}\left(Q^{2}\right)$ given by the overlap of the $\phi$-wave function. Finally, we summarize our results.

\subsection{Two-Point Function}

Our goal is to analyze form factors of vector mesons within the framework of the holographic model of QCD based on AdS/QCD correspondence. As a 4D operator on the QCD side, we take the vector current $J_{\mu}^{a}(x)=\bar{q}(x) \gamma_{\mu} t^{a} q(x)$, to which corresponds a bulk gauge field $A_{M}^{a}(x, z)$ whose boundary value is the source for $J_{\mu}^{a}(x)$. We follow the conventions of the hard-wall model [27], with the bulk fields in the background of the sliced $\mathrm{AdS}_{5}$ metric

$$
d s^{2}=\frac{1}{z^{2}}\left(\eta_{\mu \nu} d x^{\mu} d x^{\nu}-d z^{2}\right), \quad 0 \leq z \leq z_{0}
$$

where $\eta_{\mu \nu}=\operatorname{Diag}(1,-1,-1,-1)$, and $z_{0} \sim 1 / \Lambda_{Q C D}$ is the imposed IR scale. The $5 \mathrm{D}$ gauge action in $\mathrm{AdS}_{5}$ space, corresponding to $A_{M}^{a}(x, z)$, is

$$
S_{\mathrm{AdS}}=-\frac{1}{4 g_{5}^{2}} \int d^{4} x d z \sqrt{g} \operatorname{Tr}\left(F_{M N} F^{M N}\right)
$$

where $F_{M N}=\partial_{M} A_{N}-\partial_{N} A_{M}-i\left[A_{M}, A_{N}\right], A_{M}=t^{a} A_{M}^{a},\left(t^{a} \in S U(2), a=1,2,3\right)$ and $M, N=0,1,2,3, z$. Since the vector field $A_{M}^{a}(x, z)$ is taken to be non-Abelian, the 3-point function of these fields in the lowest approximation can be extracted directly from the Lagrangian.

Before calculating the 3-point function, we recall some properties of the 2-point function discussed in [27]. Consider the sliced AdS space with an IR boundary at $z=$ $z_{0}$ and UV cutoff at $z=\epsilon$ (taken to be zero at the end of the calculations). In order to calculate the current-current correlator (or 2-point function) using the AdS/CFT correspondence, one should solve equations of motion, requiring the solution at the UV boundary $(z=0)$ to coincide with the $4 \mathrm{D}$ source of the vector current, calculate $5 \mathrm{D}$ action on this solution and then vary the action (twice) with respect to the boundary source. The task is simplified when the $A_{z}=0$ gauge is imposed, and the gauge field is Fourier-transformed in $4 \mathrm{D}, A_{\mu}(x, z) \Rightarrow \tilde{A}_{\mu}(p, z)$. Then

$$
\tilde{A}_{\mu}(p, z)=\tilde{A}_{\mu}(p) \frac{V(p, z)}{V(p, \epsilon)},
$$


where $\tilde{A}_{\mu}(p)$ is the Fourier-transformed current source, and the 5D gauge field $V(p, z)$ is the so-called bulk-to-boundary propagator obeying

$$
z \partial_{z}\left(\frac{1}{z} \partial_{z} V(p, z)\right)+p^{2} V(p, z)=0
$$

The UV b.c. $\tilde{A}_{\mu}(p, \epsilon)=\tilde{A}_{\mu}(p)$ is satisfied by construction. At the IR boundary (when $z=z_{0}$ ), we follow Ref. [27] (see also Ref. [46]) and choose the Neumann b.c. $\partial_{z} V\left(p, z_{0}\right)=0$ which corresponds to the gauge invariant condition $F_{\mu z}\left(x, z_{0}\right)=0$. Evaluating the bilinear term of the action on this solution leaves only the UV surface term

$$
S_{\mathrm{AdS}}^{(2)}=-\frac{1}{2 g_{5}^{2}} \int \frac{d^{4} p}{(2 \pi)^{4}} \tilde{A}^{\mu}(p) \tilde{A}_{\mu}(p)\left[\frac{1}{z} \frac{\partial_{z} V(p, z)}{V(p, \epsilon)}\right]_{z=\epsilon} .
$$

The 2-point function of vector currents is defined by

$$
\int d^{4} x e^{i p \cdot x}\left\langle J_{\mu}^{a}(x) J_{\nu}^{b}(0)\right\rangle=\delta^{a b} \Pi_{\mu \nu}(p) \Sigma\left(p^{2}\right),
$$

where $\Pi_{\mu \nu}(p) \equiv\left(\eta_{\mu \nu}-p_{\mu} p_{\nu} / p^{2}\right)$ is the transverse projector. Varying the action (2.5) with respect to the boundary source produces

$$
\Sigma\left(p^{2}\right)=-\left.\frac{1}{g_{5}^{2}}\left(\frac{1}{z} \frac{\partial_{z} V(p, z)}{V(p, \epsilon)}\right)\right|_{z=\epsilon \rightarrow 0}
$$

(To get the tensor structure of (2.6) by a "naïve" variation, one should change $A^{\mu} A_{\mu} \rightarrow A^{\mu} \Pi_{\mu \nu}(p) A^{\nu}$ in Eq. (2.5)).

It is well known (see, e.g., [23, 46]) that two linearly independent solutions of Eq. (2.4) are given by the Bessel functions $z J_{1}(P z)$ and $z Y_{1}(P z)$, where $P \equiv \sqrt{p^{2}}$. Taking Neumann b.c. for $V(p, z)$, one obtains

$$
V(p, z)=P z\left[Y_{0}\left(P z_{0}\right) J_{1}(P z)-J_{0}\left(P z_{0}\right) Y_{1}(P z)\right]
$$

and, hence,

$$
\Sigma\left(p^{2}\right)=\frac{\pi p^{2}}{2 g_{5}^{2}}\left[Y_{0}(P z)-J_{0}(P z) \frac{Y_{0}\left(P z_{0}\right)}{J_{0}\left(P z_{0}\right)}\right]_{z=\epsilon \rightarrow 0}
$$

This expression is singular as $\epsilon \rightarrow 0$ :

$$
\Sigma\left(p^{2}\right)=\frac{1}{2 g_{5}^{2}} p^{2} \ln \left(p^{2} \epsilon^{2}\right)+\ldots
$$

By matching to QCD result for $J_{\mu}^{a}=\bar{q} \gamma_{\mu} t^{a} q$ currents one finds $g_{5}^{2}=12 \pi^{2} / N_{c}$ (cf. $[26])$. 
The two-point function $\Sigma\left(p^{2}\right)$ has poles when the denominator function $J_{0}\left(P z_{0}\right)$ has zeros, i.e., when $P z_{0}$ coincides with one of the roots $\gamma_{0, n}$ of the Bessel function $J_{0}(x)$. These poles can be explicitly displayed by incorporating the KneserSommerfeld expansion [41]

$$
\begin{aligned}
& \frac{Y_{0}\left(P z_{0}\right) J_{0}(P z)-J_{0}\left(P z_{0}\right) Y_{0}(P z)}{J_{0}\left(P z_{0}\right)} \\
& =-\frac{4}{\pi} \sum_{n=1}^{\infty} \frac{J_{0}\left(\gamma_{0, n} z / z_{0}\right)}{\left[J_{1}\left(\gamma_{0, n}\right)\right]^{2}\left(P^{2} z_{0}^{2}-\gamma_{0, n}^{2}\right)},
\end{aligned}
$$

valid for $z \leq z_{0}$ (the case we are interested in). Taking formally $z=0$ gives a logarithmically divergent series reflecting the $\ln \epsilon$ singularity of the $z=\epsilon$ expression. Thus, some kind of regularization for this divergency of the sum is implied. Under this assumption,

$$
\Sigma\left(p^{2}\right)=\frac{2 p^{2}}{g_{5}^{2} z_{0}^{2}} \sum_{n=1}^{\infty} \frac{\left[J_{1}\left(\gamma_{0, n}\right)\right]^{-2}}{p^{2}-M_{n}^{2}}
$$

where $M_{n}=\gamma_{0, n} / z_{0}$. Hence, the 2-point correlator of the hard-wall model has poles when $P$ coincides with one of $M_{n}$ 's. Given that the residues of all these poles are positive, the poles may be interpreted as bound states with $M_{n}$ 's being their masses. The coupling $f_{n}^{2}$ with which a particular resonance contributes to the total sum is determined by

$$
f_{n}^{2}=\lim _{p^{2} \rightarrow M_{n}^{2}}\left\{\left(p^{2}-M_{n}^{2}\right) \Sigma\left(p^{2}\right)\right\}
$$

This prescription agrees with the usual definition $\left\langle 0\left|J_{\mu}^{a}\right| \rho_{n}^{b}\right\rangle=\delta^{a b} f_{n} \epsilon_{\mu}$ for the vector meson decay constants. In our case,

$$
f_{n}^{2}=\frac{2 M_{n}^{2}}{g_{5}^{2} z_{0}^{2} J_{1}^{2}\left(\gamma_{0, n}\right)} .
$$

\subsection{Three-Point Function}

Consider now the trilinear term of the action calculated on the $V(q, z)$ solution:

$$
S_{\mathrm{AdS}}^{(3)}=-\frac{\epsilon_{a b c}}{2 g_{5}^{2}} \int d^{4} x \int_{\epsilon}^{z_{0}} \frac{d z}{z}\left(\partial_{\mu} A_{\nu}^{a}\right) A^{\mu, b} A^{\nu, c}
$$

A naïve variation gives the result for the 3 -point correlator $\left\langle J_{a}^{\alpha}\left(p_{1}\right) J_{b}^{\beta}\left(-p_{2}\right) J_{c}^{\mu}(q)\right\rangle$ that contains the isotopic Levi-Civita tensor $\epsilon_{a b c}$, the dynamical factor

$$
D\left(p_{1}, p_{2}, q\right) \equiv \int_{\epsilon}^{z_{0}} \frac{d z}{z} \frac{V\left(p_{1}, z\right)}{V\left(p_{1}, \epsilon\right)} \frac{V\left(p_{2}, z\right)}{V\left(p_{2}, \epsilon\right)} \frac{V(q, z)}{V(q, \epsilon)}
$$




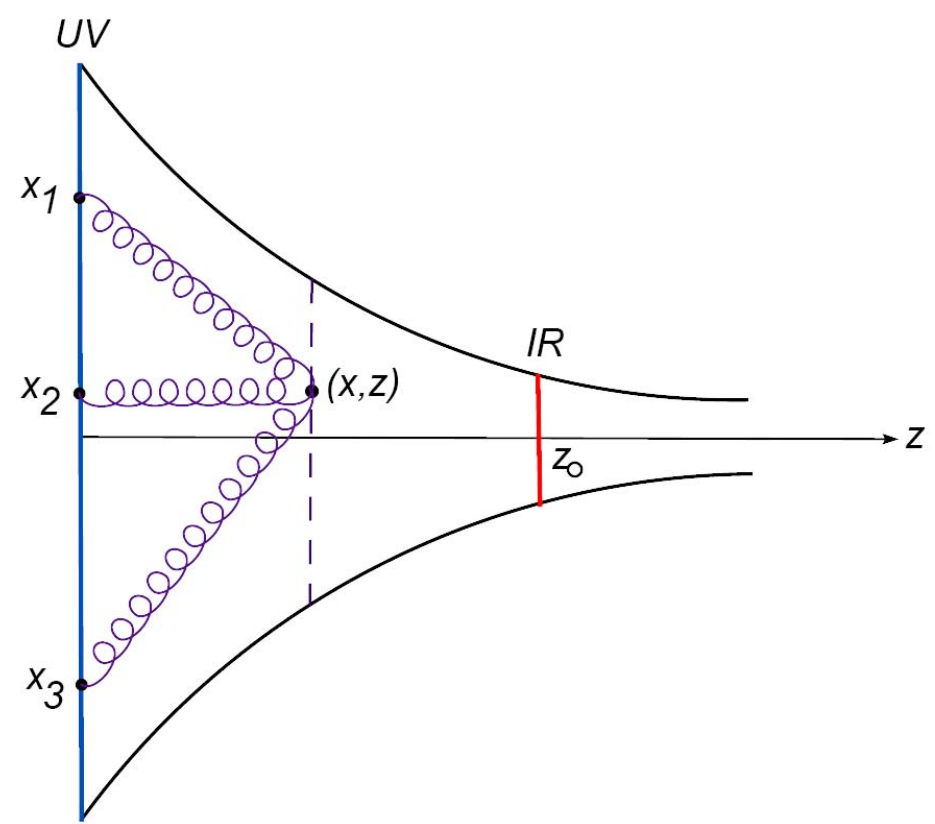

Figure 2.1: Schematic representation of the 3-gluon vertex. Vertical lines are the slices of flat four dimensional Minkowski spaces. Horizontal line is the direction along the extra fifth dimension.

and the tensor structure

$$
T^{\alpha \beta \mu}=\eta^{\alpha \mu}\left(q-p_{1}\right)^{\beta}-\eta^{\beta \mu}\left(p_{2}+q\right)^{\alpha}+\eta^{\alpha \beta}\left(p_{1}+p_{2}\right)^{\mu}
$$

familiar from the QCD 3-gluon vertex amplitude. Restoring the transverse projectors $\Pi^{\alpha \alpha^{\prime}}\left(p_{1}\right)$, etc. one can convert it into

$$
\mathcal{T}^{\alpha \beta \mu}=\eta_{\alpha \beta}\left(p_{1}+p_{2}\right)_{\mu}+2\left(\eta_{\alpha \mu} q_{\beta}-\eta_{\beta \mu} q_{\alpha}\right)
$$

For the factors corresponding to the hadronized channels, the Kneser-Sommerfeld expansion (2.11) gives

$$
\frac{V(p, z)}{V(p, 0)} \equiv \mathcal{V}(p, z)=-g_{5} \sum_{n=1}^{\infty} \frac{f_{n} \psi_{n}(z)}{p^{2}-M_{n}^{2}}
$$

where $p$ equals $p_{1}$ or $p_{2}$, and

$$
\psi_{n}(z)=\frac{\sqrt{2}}{z_{0} J_{1}\left(\gamma_{0, n}\right)} z J_{1}\left(M_{n} z\right)
$$

is the " $\psi$ wave function" obeying the same equation of motion $(2.4)$ as $V(p, z)$ (with $\left.p^{2}=M_{n}^{2}\right)$, satisfying the b.c.

$$
\psi_{n}(0)=0 \quad, \quad \partial_{z} \psi_{n}\left(z_{0}\right)=0,
$$




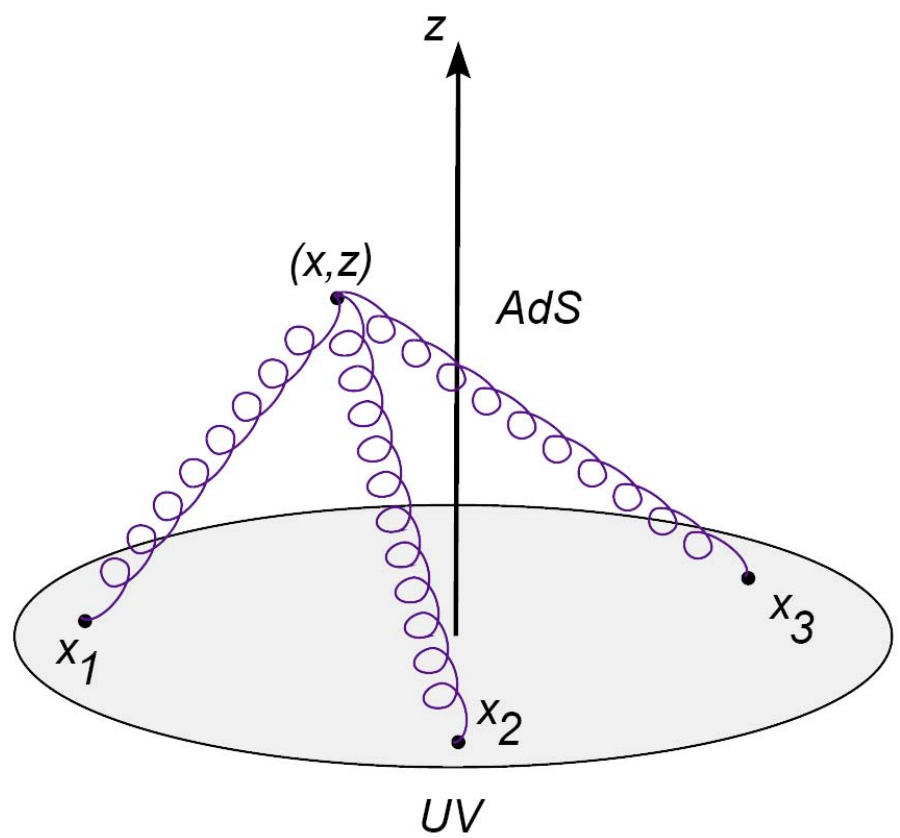

Figure 2.2: Another schematic representation of the 3-gluon vertex. The disk at the bottom is the UV boundary of the AdS space, where the four dimensional QCD resides. The IR boundary is not shown in the picture.

and normalized according to

$$
\int_{0}^{z_{0}} \frac{d z}{z}\left|\psi_{n}(z)\right|^{2}=1
$$

One remark is in order here. Since the " $\psi$ wave functions" vanish at the origin and satisfy Neumann b.c. at the IR boundary, it is impossible to establish a direct analogy between $\psi_{n}(z)$ 's and the bound state wave functions in quantum mechanics. For the latter, one would expect that they vanish at the confinement radius, while their values at the origin are proportional to the coupling constants $f_{n}$.

Taking a spacelike momentum transfer, $q^{2}=-Q^{2}$ for the $V / V$ factor of the EM current channel gives

$$
\mathcal{J}(Q, z)=Q z\left[K_{1}(Q z)+I_{1}(Q z) \frac{K_{0}\left(Q z_{0}\right)}{I_{0}\left(Q z_{0}\right)}\right],
$$

the non-normalizable mode with Neumann b.c. (see also Ref. [46]). This factor can also be written as a sum of monopole contributions from the infinite tower of vector mesons:

$$
\mathcal{J}(Q, z)=g_{5} \sum_{m=1}^{\infty} \frac{f_{m} \psi_{m}(z)}{Q^{2}+M_{m}^{2}},
$$

This decomposition, discussed in Ref. [46], directly follows from Eq. (2.18). Incorporating the representation for the bulk-boundary propagators given above we 
obtain

$$
T\left(p_{1}^{2}, p_{2}^{2}, Q^{2}\right)=\sum_{n, k=1}^{\infty} \frac{f_{n} f_{k} F_{n k}\left(Q^{2}\right)}{\left(p_{1}^{2}-M_{n}^{2}\right)\left(p_{2}^{2}-M_{k}^{2}\right)},
$$

where $T\left(p_{1}^{2}, p_{2}^{2}, Q^{2}\right)=D\left(p_{1}, p_{2}, q\right) / g_{5}^{2}$, and

$$
F_{n k}\left(Q^{2}\right)=\int_{0}^{z_{0}} \frac{d z}{z} \mathcal{J}(Q, z) \psi_{n}(z) \psi_{k}(z)
$$

correspond to form factors for $n \rightarrow k$ transitions. This expression was also written in Ref. [46] for form factors considered there.

\subsection{Wave Functions}

The formulas obtained above using explicit properties of the Bessel functions in the form of Kneser-Sommerfeld expansions, can also be derived from the general formalism of Green's functions. In particular, the Green's function for Eq. (2.4) can be written as

$$
G\left(p ; z, z^{\prime}\right)=\sum_{n=1}^{\infty} \frac{\psi_{n}(z) \psi_{n}\left(z^{\prime}\right)}{p^{2}-M_{n}^{2}}
$$

where $\psi_{n}(z)$ 's are the normalized wave functions (5.5) that satisfy the SturmLiouville equation (2.4) with $p^{2}=M_{n}^{2}$ and Neumann b.c. (2.20). As discussed in Ref. [26], the bulk-to-boundary propagator is related to the Green's function by

$$
\mathcal{V}\left(p, z^{\prime}\right)=-\left[\frac{1}{z} \partial_{z} G\left(p ; z, z^{\prime}\right)\right]_{z=\epsilon \rightarrow 0},
$$

and the two-point function $\Sigma\left(P^{2}\right)$ is obtained from the Green's function using Eqs. (2.7),(2.27)

$$
\Sigma\left(P^{2}\right)=\frac{1}{g_{5}^{2}}\left[\frac{1}{z^{\prime}} \partial_{z^{\prime}}\left[\frac{1}{z} \partial_{z} G\left(p ; z, z^{\prime}\right)\right]\right]_{z, z^{\prime}=\epsilon \rightarrow 0} .
$$

Accordingly, the coupling constants are related to the $\psi$ wave functions by

$$
f_{n}=\left[\frac{1}{z} \partial_{z} \psi_{n}(z)\right]_{z=0}
$$

(cf. $[46,26])$. In view of this relation, it makes sense to introduce " $\phi$ wave functions"

$$
\phi_{n}(z) \equiv \frac{1}{M_{n} z} \partial_{z} \psi_{n}(z)=\frac{\sqrt{2}}{z_{0} J_{1}\left(\gamma_{0, n}\right)} J_{0}\left(M_{n} z\right)
$$

which give the couplings $g_{5} f_{n} / M_{n}$ as their values at the origin. In this respect, the " $\phi$ wave functions" are analogous to the bound state wave functions in quantum mechanics. 


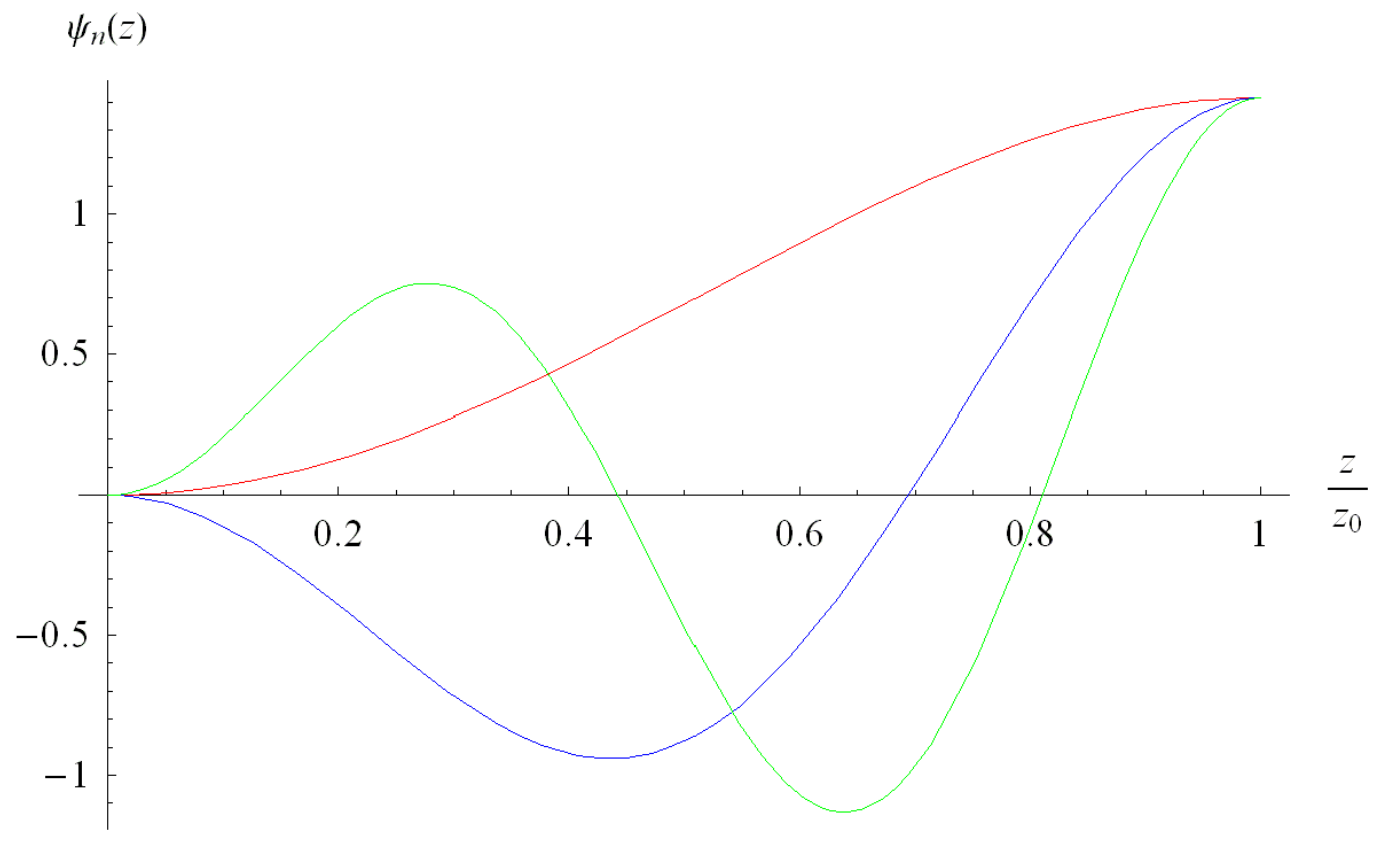

Figure 2.3: Eigenfunctions $\psi_{n}(z)$ as function of $z / z_{0}$ for first three modes. The number of modes of each curve determines $n$.

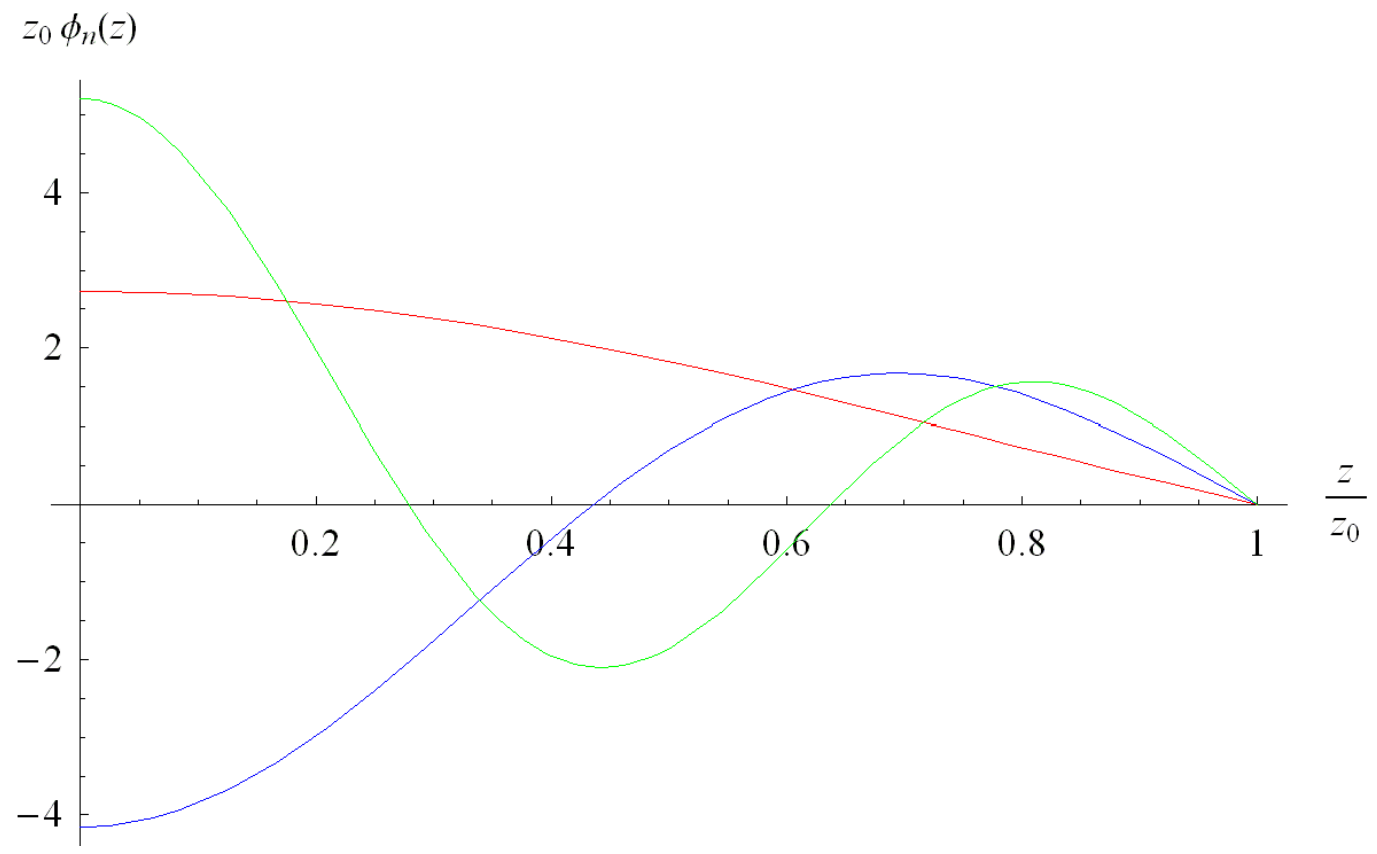

Figure 2.4: Functions $z_{0} \phi_{n}(z)$ as function of $z / z_{0}$. The color on the curves corresponds to the same modes as in the previous plot. 
Moreover, these functions satisfy Dirichlet b. c. $\phi_{n}\left(z_{0}\right)=0$ and are normalized by

$$
\int_{0}^{z_{0}} d z z\left|\phi_{n}(z)\right|^{2}=1
$$

which strengthens this analogy. However, the elastic form factors $F_{n n}\left(Q^{2}\right)$ are given by the integrals

$$
F_{n n}\left(Q^{2}\right)=\int_{0}^{z_{0}} \frac{d z}{z} \mathcal{J}(Q, z)\left|\psi_{n}(z)\right|^{2}
$$

involving $\psi$ rather than $\phi$ wave functions. In fact, due to the basic equation (2.4), $\psi_{n}(z)$ wave functions can be expressed in terms of $\phi_{n}(z)$ as

$$
\psi_{n}(z)=-\frac{z}{M_{n}} \partial_{z} \phi_{n}(z)
$$

and we can rewrite the form factor integral as

$$
\begin{aligned}
F_{n n}\left(Q^{2}\right)= & \int_{0}^{z_{0}} d z z \mathcal{J}(Q, z)\left|\phi_{n}(z)\right|^{2} \\
& +\frac{1}{M_{n}} \int_{0}^{z_{0}} d z \phi_{n}(z) \psi_{n}(z) \partial_{z} \mathcal{J}(Q, z) .
\end{aligned}
$$

Note, that the nonnormalizable mode

$$
\frac{1}{z} \partial_{z} \mathcal{J}(Q, z)=-Q^{2}\left[K_{0}(Q z)-I_{0}(Q z) \frac{K_{0}\left(Q z_{0}\right)}{I_{0}\left(Q z_{0}\right)}\right]
$$

corresponds to equation whose solutions are the functions $J_{0}\left(M_{n} z\right)$ satisfying Dirichlet b.c. at $z=z_{0}$. Expressing $\phi_{n}(z)$ in terms of $\partial_{z} \psi_{n}(z)$, integrating $\left|\psi_{n}(z)\right|^{2}$ by parts and using equation (2.4) for $\mathcal{J}(Q, z)$ gives

$$
\begin{aligned}
F_{n n}\left(Q^{2}\right)= & \int_{0}^{z_{0}} d z z \mathcal{J}(Q, z)\left|\phi_{n}(z)\right|^{2} \\
& -\frac{Q^{2}}{2 M_{n}^{2}} \int_{0}^{z_{0}} \frac{d z}{z} \mathcal{J}(Q, z)\left|\psi_{n}(z)\right|^{2} .
\end{aligned}
$$

The second term contains the original integral for $F_{n n}\left(Q^{2}\right)$, and we obtain

$$
F_{n n}\left(Q^{2}\right)=\frac{1}{1+Q^{2} / 2 M_{n}^{2}} \int_{0}^{z_{0}} d z z \mathcal{J}(Q, z)\left|\phi_{n}(z)\right|^{2} .
$$

Notice, that the normalizable modes $\phi_{n}(z)$ in this expression correspond to Dirichlet b.c., while the nonnormalizable mode $\mathcal{J}(Q, z)$ was obtained using the Neumann ones.

Thus, we managed to get the expression for $F_{n n}\left(Q^{2}\right)$ form factors that contains $\phi$ instead of $\psi$ wave functions. However, it contains an extra factor $1 /\left(1+Q^{2} / 2 M_{n}^{2}\right)$, which brings us to the issue of the different form factors of the $\rho$-meson and kinematic factors associated with them. 

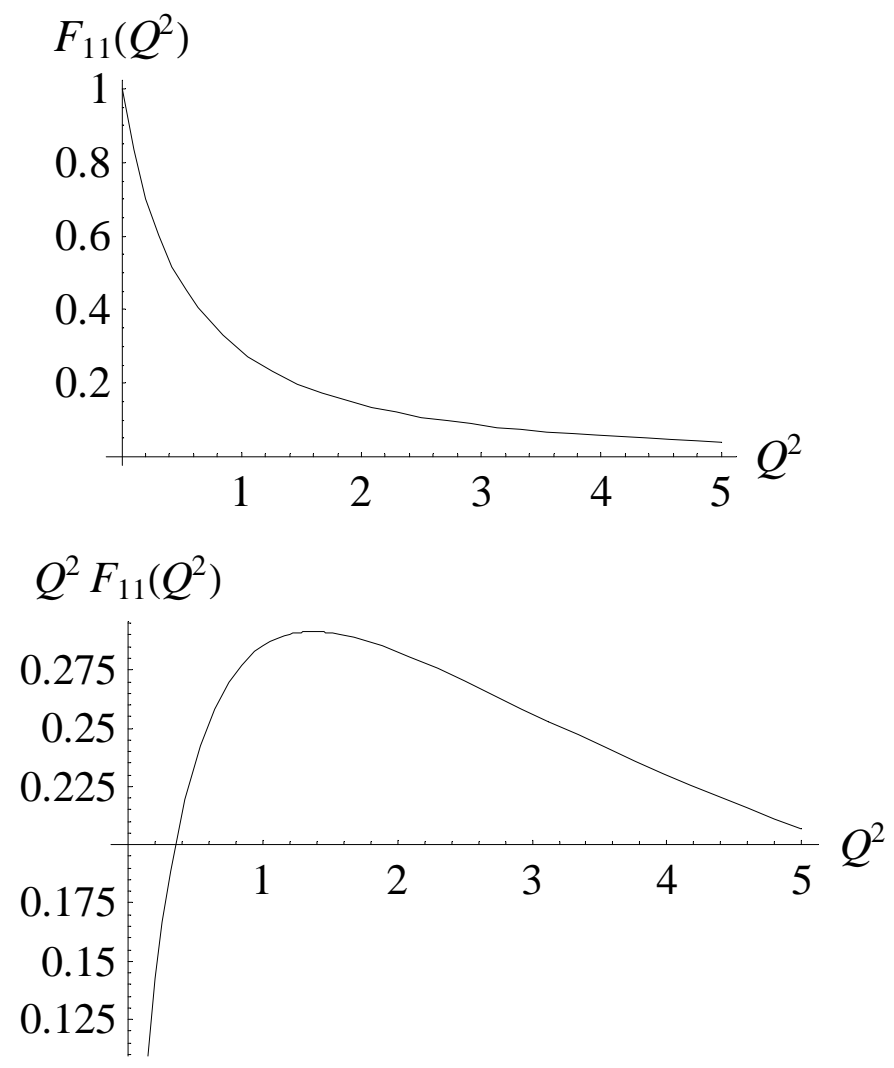

Figure 2.5: Plots of $F_{11}\left(Q^{2}\right)$ and $Q^{2} F_{11}\left(Q^{2}\right)$ as a function of $Q^{2}\left(\mathrm{GeV}^{2}\right)$.

\subsection{Form Factors}

Our result for the form factor contains only one function for each $n \rightarrow k$ transition, in particular $F_{n n}\left(Q^{2}\right)$ in the diagonal case. However, the general expression for the EM vertex of a spin-1 particle of mass $M$ can be written (assuming $P$ - and $T$-invariance) in terms of three form factors (see, e.g., [42], our $G_{2}$ is their $G_{2}-G_{1}$ ):

$$
\begin{aligned}
& \left\langle\rho^{+}\left(p_{2}, \epsilon^{\prime}\right)\left|J_{\mathrm{EM}}^{\mu}(0)\right| \rho^{+}\left(p_{1}, \epsilon\right)\right\rangle \\
& =-\epsilon_{\beta}^{\prime} \epsilon_{\alpha}\left[\eta^{\alpha \beta}\left(p_{1}^{\mu}+p_{2}^{\mu}\right) G_{1}\left(Q^{2}\right)\right. \\
& +\left(\eta^{\mu \alpha} q^{\beta}-\eta^{\mu \beta} q^{\alpha}\right)\left(G_{1}\left(Q^{2}\right)+G_{2}\left(Q^{2}\right)\right) \\
& \left.\quad-\frac{1}{M^{2}} q^{\alpha} q^{\beta}\left(p_{1}^{\mu}+p_{2}^{\mu}\right) G_{3}\left(Q^{2}\right)\right] .
\end{aligned}
$$

Comparing the tensor structure of this expression with (2.17), we conclude that the hard-wall model predicts $G_{1}^{(n)}\left(Q^{2}\right)=G_{2}^{(n)}\left(Q^{2}\right)=F_{n n}\left(Q^{2}\right)$, and $G_{3}^{(n)}\left(Q^{2}\right)=0$ for form factors $G_{i}^{(n)}\left(Q^{2}\right)$ of $n^{\text {th }}$ bound state. It was argued (see [46]) that this is a 
general feature of AdS/QCD models for the $\rho$-meson form factors. Since $\mathcal{J}(Q=$ $0, z)=1$, the diagonal form factors $F_{n n}\left(Q^{2}\right)$ in the hard-wall are normalized to unity, while the nondiagonal ones vanish for $Q^{2}=0$ (the functions $\psi_{n}(z)$ are orthonormal on $\left.\left[0, z_{0}\right]\right)$.

The form factors $G_{i}$ are related to electric $G_{C}$, magnetic $G_{M}$ and quadrupole $G_{Q}$ form factors by

$$
\begin{aligned}
G_{C} & =G_{1}+\frac{Q^{2}}{6 M^{2}} G_{Q}, \quad G_{M}=G_{1}+G_{2}, \\
G_{Q} & =\left(1+\frac{Q^{2}}{4 M^{2}}\right) G_{3}-G_{2} .
\end{aligned}
$$

For these form factors, hard-wall gives

$$
\begin{aligned}
& G_{Q}^{(n)}\left(Q^{2}\right)=-F_{n n}\left(Q^{2}\right), G_{M}^{(n)}\left(Q^{2}\right)=2 F_{n n}\left(Q^{2}\right), \\
& G_{C}^{(n)}\left(Q^{2}\right)=\left(1-\frac{Q^{2}}{6 M^{2}}\right) F_{n n}\left(Q^{2}\right) .
\end{aligned}
$$

For $Q^{2}=0$, it correctly reproduces the unit electric charge of the meson, and "predicts" $\mu \equiv G_{M}(0)=2$ for the magnetic moment and $D \equiv G_{Q}(0) / M^{2}=-1 / M^{2}$ for the quadrupole moment, which are just the canonical values for a pointlike vector particle [60].

Another interesting combination of form factors

$$
\mathcal{F}\left(Q^{2}\right)=G_{1}\left(Q^{2}\right)+\frac{Q^{2}}{2 M^{2}} G_{2}\left(Q^{2}\right)-\left(\frac{Q^{2}}{2 M^{2}}\right)^{2} G_{3}\left(Q^{2}\right)
$$

appears if one takes the " +++ " component of the 3-point correlator (obtained, e.g., by convoluting it with $n_{\alpha} n_{\beta} n_{\mu}$, where $n^{2}=0,\left(n p_{1}\right)=1,(n q)=0$ [35]). The hard-wall model result $(2.37)$ for $\mathcal{F}\left(Q^{2}\right)$ is particularly simple:

$$
\mathcal{F}_{n n}\left(Q^{2}\right)=\int_{0}^{z_{0}} d z z \mathcal{J}(Q, z)\left|\phi_{n}(z)\right|^{2} .
$$

Thus, it is the form factors $\mathcal{F}_{n n}\left(Q^{2}\right)$ that are the most direct analogues of diagonal bound state form factors in quantum mechanics.

\subsection{Low- $Q^{2}$ Behavior}

Our expression for $\mathcal{F}_{n n}\left(Q^{2}\right)$ is close to that proposed for a generic meson form factor in the holographic model of Ref. [30]. There, the authors used $\mathcal{K}(Q z) \equiv$ $Q z K_{1}(Q z)$ as the $q$-channel factor. Indeed, the difference between $\mathcal{J}(Q, z)$ and $\mathcal{K}(Q z)$ is exponentially small when $Q z_{0} \gg 1$, but the two functions radically differ in the region of small $Q^{2}$, where the function $\mathcal{K}(Q z)$ displays the logarithmic branch singularity

$$
\mathcal{K}(Q z)=1-\frac{z^{2} Q^{2}}{4}\left[1-2 \gamma_{E}-\ln \left(Q^{2} z^{2} / 4\right)\right]+\mathcal{O}\left(Q^{4}\right)
$$



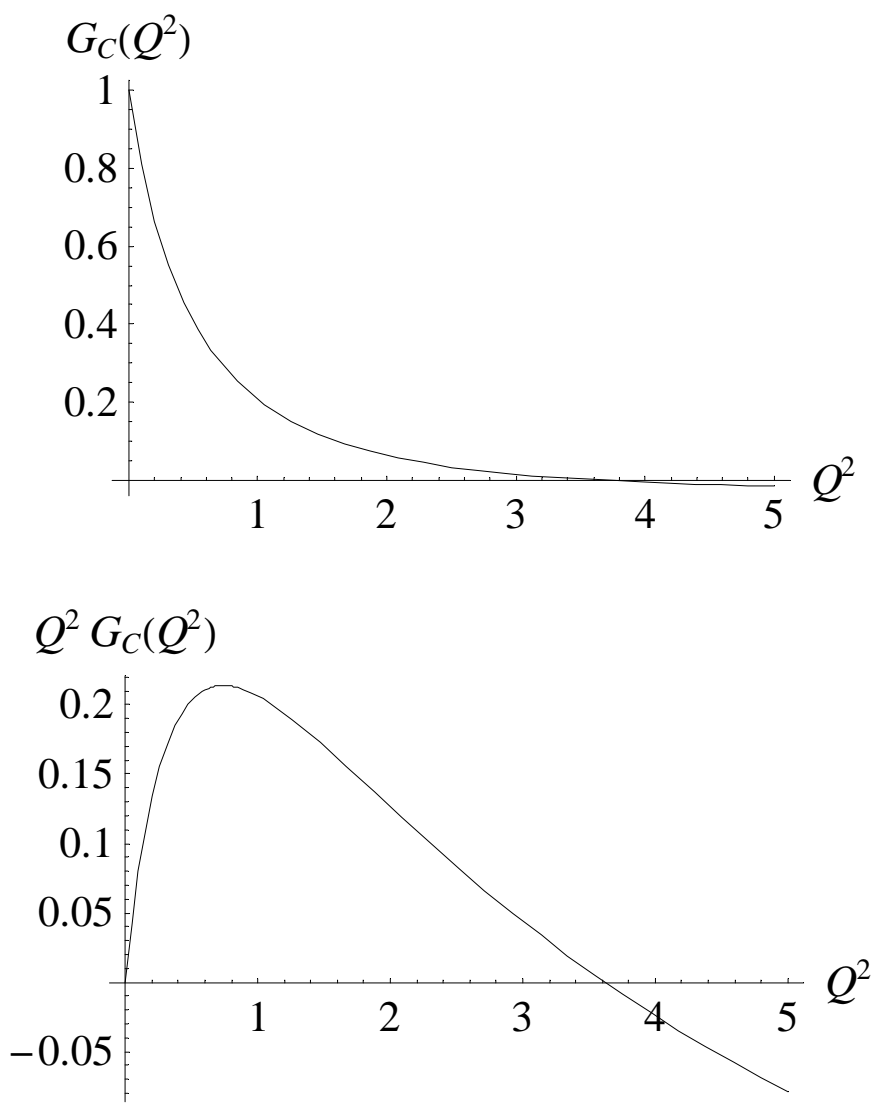

Figure 2.6: Plots of $G_{C}\left(Q^{2}\right)$ and $Q^{2} G_{C}\left(Q^{2}\right)$ as a function of $Q^{2}\left(\mathrm{GeV}^{2}\right)$.

that leads to incorrect infinite slope at $Q^{2}=0$. To implant the AdS/QCD information about the hadron spectrum in the $q$-channel one should use $\mathcal{J}(Q, z)$ that corresponds to a tower of bound states in the $q$-channel. The lowest singularity in this case is located at $Q^{2}=-M_{1}^{2}$. Since it is separated by a finite gap from zero, the form factor slopes at $Q^{2}=0$ are finite.

To analyze the form factor behavior in the $Q z_{0} \ll 1$ limit, we expand

$$
\left.\mathcal{J}(Q, z)\right|_{Q z_{0} \ll 1}=1-\frac{z^{2} Q^{2}}{4}\left[1-\ln \frac{z^{2}}{z_{0}^{2}}\right]+\mathcal{O}\left(Q^{4}\right) .
$$

As expected, the result is analytic in $Q^{2}$. For the lowest transition (i.e., for the $\rho$-meson form factor), explicit numbers are as follows:

$$
\mathcal{F}_{11}\left(Q^{2}\right) \approx 1-0.692 \frac{Q^{2}}{M^{2}}+0.633 \frac{Q^{4}}{M^{4}}+\mathcal{O}\left(Q^{6}\right)
$$

where $M=M_{1}=m_{\rho}$. Another small- $Q^{2}$ expansion

$$
F_{11}\left(Q^{2}\right) \approx 1-1.192 \frac{Q^{2}}{M^{2}}+1.229 \frac{Q^{4}}{M^{4}}+\mathcal{O}\left(Q^{6}\right)
$$


can be either calculated from the original expression (2.32) involving $\psi$-functions or by dividing $\mathcal{F}_{11}\left(Q^{2}\right)$ by $\left(1+Q^{2} / 2 M^{2}\right)$. The latter approach easily explains the difference in slopes of these two form factors at $Q^{2}=0$. Finally, for the electric form factor, we obtain

$$
G_{C}^{(1)}\left(Q^{2}\right) \approx 1-1.359 \frac{Q^{2}}{M^{2}}+1.428 \frac{Q^{4}}{M^{4}}+\mathcal{O}\left(Q^{6}\right)
$$

For the electric radius of the $\rho$-meson this gives

$$
\left\langle r_{\rho}^{2}\right\rangle_{C}=0.53 \mathrm{fm}^{2}
$$

the value that is very close to the recent result $\left(0.54 \mathrm{fm}^{2}\right)$ obtained within the Dyson-Schwinger equations (DSE) approach [43]. Lattice gauge calculations [44] indicate a similar value in the $m_{\pi}^{2} \rightarrow 0$ limit.

\subsection{Vector Meson Dominance Patterns}

Numerically, the result $1.359 / M^{2}$ for the slope of $G_{C}^{(1)}\left(Q^{2}\right)$ is larger than the simple VMD expectation $1 / M^{2}$. In fact, a part of this larger value is due to the factor $\left(1-Q^{2} / 6 M^{2}\right)$ relating $G_{C}^{(1)}\left(Q^{2}\right)$ and $F_{11}\left(Q^{2}\right)$, which is kinematic to some extent. The $F_{11}\left(Q^{2}\right)$ form factor, however, can be written in the generalized VMD representation (cf. [46])

$$
F_{11}\left(Q^{2}\right)=\sum_{m=1}^{\infty} \frac{F_{m, 11}}{1+Q^{2} / M_{m}^{2}},
$$

with the coefficients $F_{m, 11}$ given by the overlap integrals

$$
F_{m, 11}=4 \int_{0}^{1} d \xi \xi^{2} \frac{J_{1}\left(\gamma_{0, m} \xi\right) J_{1}^{2}\left(\gamma_{0,1} \xi\right)}{\gamma_{0, m} J_{1}^{2}\left(\gamma_{0, m}\right) J_{1}^{2}\left(\gamma_{0,1}\right)},
$$

apparently having a purely dynamical origin. The coefficients $F_{m, 11}$ satisfy the sum rule

$$
\sum_{m=1}^{\infty} F_{m, 11}=1
$$

that provides correct normalization $F_{11}\left(Q^{2}=0\right)=1$. Numerically, the unity value of the form factor $F_{11}\left(Q^{2}\right)$ for $Q^{2}=0$ is dominated by the first bound state that gives 1.237. The second bound state makes a sizable correction by -0.239 , while adding a small 0.002 contribution from the third bound state fine-tunes 1 beyond the $10^{-3}$ accuracy. Contributions from higher bound states to the form factor normalization are negligible at this precision.

The slope of $F_{11}\left(Q^{2}\right)$ at $Q^{2}=0$ is given by the sum of $F_{m, 11} / M_{m}^{2}$ coefficients. Now, the dominance of the first bound state is even more pronounced: the $Q^{2}$ coefficient $1.192 / M^{2}$ in Eq. (2.46) is basically contributed by the first bound state that gives $1.237 / M^{2}$, with small $-0.045 / M^{2}$ correction from the second bound state. Other resonances are not visible at the three-digit precision. 
Thus, for small $Q^{2}$, the hard-wall model predicts a rather peculiar pattern of VMD for $F_{11}\left(Q^{2}\right)$ (observed originally in Ref. [46] for a form factor considered there): strong dominance of the first $q$-channel bound state, whose coupling $F_{1,11}$ exceeds 1 , with the second resonance (having the negative coupling $F_{2,11}$ ) compensating this excess.

Similarly, the $\mathcal{F}_{11}\left(Q^{2}\right)$ form factor has the generalized VMD representation with coefficients $\mathcal{F}_{m, 11}$ given by the overlap integrals

$$
\mathcal{F}_{m, 11}=4 \int_{0}^{1} d \xi \xi^{2} \frac{J_{1}\left(\gamma_{0, m} \xi\right) J_{0}^{2}\left(\gamma_{0,1} \xi\right)}{\gamma_{0, m} J_{1}^{2}\left(\gamma_{0, m}\right) J_{1}^{2}\left(\gamma_{0,1}\right)} .
$$

Now, $\mathcal{F}_{1,11} \approx 0.619, \mathcal{F}_{2,11} \approx 0.391, \mathcal{F}_{3,11} \approx-0.012, \mathcal{F}_{4,11} \approx 0.002$, etc. In this case also, the value of the $\mathcal{F}_{11}\left(Q^{2}\right)$ form factor for $Q^{2}=0$ is dominated by the first two bound states. For the slope of the form factor at $Q^{2}=0$, the dominance of the first bound state is again more pronounced: the $Q^{2}$ coefficient $0.692 / M^{2}$ in Eq. (2.45) is basically contributed by the first bound state that gives $0.619 / M^{2}$, with a small $0.074 / M^{2}$ correction from the second bound state and a tiny $-0.001 / M^{2}$ contribution from the third one.

Thus, for $\mathcal{F}_{11}\left(Q^{2}\right)$, hard-wall gives again a two-resonance dominance pattern, with the coupling $\mathcal{F}_{2,11}$ of the second resonance being now just somewhat smaller than the coupling $\mathcal{F}_{1,11}$ of the first resonance, both being positive. The relation between the two VMD patterns follows from Eq. (2.37):

$$
F_{m, 11}=\frac{\mathcal{F}_{m, 11}}{1-M_{m}^{2} / 2 M_{1}^{2}} .
$$

In particular, it gives $F_{1,11}=2 \mathcal{F}_{1,11}$, and negative sign for $F_{2,11}$. It also determines that if higher coefficients $\mathcal{F}_{m, 11}$ are small then $F_{m, 11}$ 's are even smaller.

\subsection{Large- $Q^{2}$ Behavior}

Eq. (2.37) tells us that asymptotically $F_{11}\left(Q^{2}\right)$ is suppressed by a power of $1 / Q^{2}$ compared to $\mathcal{F}_{11}\left(Q^{2}\right)$, which is known to behave like $1 / Q^{2}$ for large $Q^{2}[30,35]$. The absence of $1 / Q^{2}$ term in the asymptotic expansion for $F_{11}\left(Q^{2}\right)$ means that the coefficients $F_{m, 11}$ defined in Eq. (2.49) satisfy the "superconvergence" relation

$$
\sum_{m=1}^{\infty} M_{m}^{2} F_{m, 11}=0
$$

reflecting a "conspiracy" [46] between the poles. Writing $M_{m}^{2} F_{m, 11} \equiv A_{m} M^{2}$, we obtain that $A_{1} \approx 1.237, A_{2} \approx-1.261, A_{3} \approx 0.027$ (our results for the ratios $A_{2} / A_{1}$, $A_{3} / A_{1}$ agree with the calculation of Ref. [46]). Again, the sum rule is practically saturated by the first two bound states, which give contributions that are close in magnitude but opposite in sign.

In the case of $\mathcal{F}\left(Q^{2}\right)$, the two lowest bound states both give positive $\mathcal{O}\left(1 / Q^{2}\right)$ contributions at large $Q^{2}$. In Ref. [35], it was shown that the asymptotic normalization of $\mathcal{F}_{11}\left(Q^{2}\right)$ exceeds the VMD expectation $M_{1}^{2} / Q^{2}$ by a factor of 2.566 . We can 
infer this normalization from the values of the coefficients $\mathcal{F}_{m, 11}$ defined in Eq. (2.52). Writing $M_{m}^{2} \mathcal{F}_{m, 11} \equiv \mathcal{A}_{m} M_{1}^{2}$, we obtain that $\mathcal{A}_{1} \approx 0.619, \mathcal{A}_{2} \approx 2.061, \mathcal{A}_{3} \approx-0.150$, $\mathcal{A}_{4} \approx 0.054$. Note, that the total result is dominated by the second bound state, which is responsible for about $80 \%$ of the value. The lowest bound state contributes only about $25 \%$, while the higher states give just small corrections.

It is worth noting that the large- $Q^{2}$ behavior of $\mathcal{F}_{11}\left(Q^{2}\right)$ is determined by the large- $Q z_{0}$ form of $\mathcal{J}(Q, z)$ : it can be (and was) calculated using $\mathcal{K}(Q z)$, the free-field version of $\mathcal{J}(Q, z)$. As a result, the value of the asymptotic coefficient (2.566 in case of $\left.\mathcal{F}_{11}\left(Q^{2}\right)\right)$ is settled by the sum rule

$$
\sum_{m=1}^{\infty} M_{m}^{2} \mathcal{F}_{m, 11}=\left|\phi_{1}(0)\right|^{2} \int_{0}^{\infty} d \chi \chi^{2} K_{1}(\chi)=2\left|\phi_{1}(0)\right|^{2}
$$

that should be satisfied by any set of coefficients $\mathcal{F}_{m, 11}$. A particular distribution of "2.566" among the bound states is governed by the specific $q$-channel dynamics (in our case, by the choice of the Neumann b.c. for $\mathcal{J}(Q, z)$ at $z=z_{0}$ ). Thus, in the dynamics described by $\mathcal{J}(Q, z)$, the large value of the asymptotic coefficient is explained by large contribution due to the second bound state.

It was shown in Ref. [35] that the asymptotic $1 / Q^{2}$ behavior for $\mathcal{F}_{11}\left(Q^{2}\right)$ is established only for $Q^{2} \sim 10 \mathrm{GeV}^{2}$, and one may question the applicability of the hard-wall model for such large $Q^{2}$. The discussion of this problem, however, is beyond the scope of the present work.

\subsection{Summary}

In this chapter, we described the formalism that allows one to study form factors of vector mesons in the holographic QCD model of Refs. [27, 26, 28] (hard-wall model). An essential ingredient of our approach is a systematic use of the Kneser-Sommerfeld representation that explicitly displays the poles of two- and three-point functions and describes the structure of the corresponding bound states by eigenfunctions of the 5D equation of motion, the " $\psi$ wave functions". These functions vanish at $z=0$ and satisfy Neumann b.c. at $z=z_{0}$, which prevents a direct analogy with bound state wave functions in quantum mechanics. To this end, we introduced an alternative description in terms of " $\phi$ wave functions" that satisfy Dirichlet b.c. at $z=z_{0}$ and have finite values at $z=0$ which determine bound state couplings $f_{n} / M_{n}$. Thus, the $\phi$ wave functions have the properties necessary for the light-cone interpretation proposed in Ref. [30] and discussed also in Ref.[35].

Analyzing the three-point function, we derived expressions for bound state form factors both in terms of $\psi$ and $\phi$ wave functions, and obtained specific predictions for form factor behavior at small and large values of the invariant momentum transfer $Q^{2}$. In particular, we calculated the electric radius of the $\rho$ meson, and obtained the value $\left\langle r_{\rho}^{2}\right\rangle_{C}=0.53 \mathrm{fm}^{2}$ that practically coincides with the recent result [43] obtained within the DSE approach. Our result is also consistent with the $m_{\pi}^{2} \rightarrow 0$ extrapolation of the recent lattice gauge calculation [44]. 
We derived a generalized VMD representation both for the $F_{11}\left(Q^{2}\right)$ form factor (the expression for which coincides with a model $\rho$-meson form factor considered in Ref. [46]) and for the $\mathcal{F}_{11}\left(Q^{2}\right)$ form factor introduced in the Ref. [36] (present work), and demonstrated that hard-wall model predicts a very specific VMD pattern, in which these form factors are essentially given by contributions due to the first two bound states in the $Q^{2}$-channel, with the higher bound states playing a negligible role. We showed that, while the form factor slopes at $Q^{2}=0$ in this picture are dominated by the first bound state, the second bound state plays a crucial role in the large- $Q^{2}$ asymptotic limit. In particular, it provides the bulk part of the negative contribution necessary to cancel the naïve VMD $1 / Q^{2}$ asymptotics for the $F_{11}\left(Q^{2}\right)$ form factor (corresponding to the overlap integral involving the $\psi$ functions), and it dominates the asymptotic $1 / Q^{2}$ behavior of the $\mathcal{F}\left(Q^{2}\right)$ form factor (given by the overlap of the $\phi$ functions).

A possible future application of our approach is the analysis of bound state form factors in the model of Ref. [32] in which the hard-wall boundary conditions at the $z=z_{0}$ IR boundary are substituted by an oscillator-type potential. This model provides the $M_{n}^{2} \sim n \Lambda^{2}$ asymptotic behavior of the spectrum of highly excited mesons, which is more consistent with the semiclassical limit of QCD [45] than the $M_{n}^{2} \sim n^{2} \Lambda^{2}$ result of the hard-wall model. 


\section{Chapter 3}

\section{Form Factors in Holographic Model with Linear Confinement}

\subsection{Introduction}

In the hard-wall model, the confinement is modeled by the hard-wall cutting off the AdS space along the extra fifth dimension at some finite value $z=z_{0}$. The solutions of the relevant eigenvalue equation are given by the Bessel functions, and masses of bound states are given by the roots $M_{n}=\gamma_{0, n} / z_{0}$ of $J_{0}\left(M z_{0}\right)$. As a result, the masses of higher excitations behave like $M_{n}^{2} \sim n^{2}$. It was argued $[32,45]$ that, instead, one should expect $M_{n}^{2} \sim n$ behavior. This connection can be derived from semiclassical arguments $[47,45]$. An explicit AdS/QCD model which gives such a linear behavior was proposed in Ref. [32]. The hard-wall boundary conditions in this model are substituted by an oscillator-type potential providing a soft IR cut-off in the action integral (for this reason, it will be referred to as "soft-wall model").

In the present chapter*, we study form factors and wave functions of vector mesons within the framework of the soft-wall model formulated in Ref. [32], and compare the results with those we obtained in Ref. [36] investigating the hard-wall model. To this end, we extend the approach developed in Ref. [36]. We start with recalling the basics of the soft-wall model and some results obtained in Ref. [32], in particular, the form of the relevant action, the eigenvalue equation for bound states and its solution. We derive a useful integral representation for the bulk-toboundary propagator $\mathcal{V}(p, z)$ that allows us to write $\mathcal{V}(p, z)$ as an explicit expansion over bound state poles with the $z$-dependence of each pole contribution given by " $\psi$ wave functions" that are eigenfunctions of the $5 \mathrm{D}$ equation of motion. Then we show that the same representation can be obtained from the general formalism of Green's functions. However, as we already emphasized in Ref. [36], the $\psi_{n}(z)$ wave functions are not direct analogues of the usual quantum-mechanical wave functions. In particular, a meson coupling constant $f_{n}$ is obtained from the derivative of $\psi_{n}(z)$ at $z=0$ rather than from its value at this point. To this end, we introduce " $\phi$ wave functions" which look more like wave functions of oscillator bound states in

\footnotetext{
*The main results from the Ref. [37] are printed by permission from the APS, see Appendix.
} 
quantum mechanics. Their values at $z=0$ give the bound state couplings $g_{5} f_{n} / M_{n}$, they exponentially decrease with $z^{2}$, and thus they have properties necessary for the light-cone interpretation of AdS/QCD results proposed in Ref. [30]. Further, we study the three-point function $\langle J J J\rangle$ and obtain expressions for transition form factors that involves $\psi$ wave functions and the nonnormalizable mode factor $\mathcal{J}(Q, z)$. The latter is written as a sum over all bound states in the channel of electromagnetic current, which gives an analogue of generalized vector meson dominance (VMD) representation for hadronic form factors. We also show that it is possible to rewrite form factors in terms of $\phi$ functions. Then we formulate predictions for $\rho$-meson form factors, and analyze these predictions in the regions of small and large $Q^{2}$. In particular, our formalism allows us to calculate the $\rho$-meson electric radius, and the radii of higher excited states. It is also shown that, for the basic $\rho$-meson form factor $\mathcal{F}\left(Q^{2}\right)$ given by the overlap of the $\phi$ wave functions, the soft-wall model predicts exact VMD pattern, when just one lowest bound state in the $Q^{2}$-channel contributes. For another $\rho$-meson form factor $F\left(Q^{2}\right)$, which is given by the overlap of the $\psi$ wave functions, a two-resonance dominance is established, with only two lowest bound states in the $Q^{2}$-channel contributing. We compare our results obtained in the softwall model with those derived in the hard-wall model studies performed in Ref. [36]. Finally, we summarize our results obtained in this chapter.

\subsection{Preliminaries}

We consider the gravity background with a smooth cutoff that was proposed in Ref. [32] instead of a hard-wall infrared (IR) cutoff. In this case, the only background fields are dilaton $\chi(z)=z^{2} \kappa^{2}$ and metric $g_{M N}$. The metric can be written as

$$
g_{M N} d x^{M} d x^{N}=\frac{1}{z^{2}}\left(\eta_{\mu \nu} d x^{\mu} d x^{\nu}-d z^{2}\right)
$$

where $\eta_{\mu \nu}=\operatorname{Diag}(1,-1,-1,-1)$ and $\mu, \nu=(0,1,2,3), M, N=(0,1,2,3, z)$. To determine the spectrum of vector mesons, one needs the quadratic part of the action

$$
S_{\mathrm{AdS}}=-\frac{1}{4 g_{5}^{2}} \int d^{4} x \frac{d z}{z} e^{-\chi} \operatorname{Tr}\left(F_{M N} F^{M N}\right)
$$

where $F_{M N}=\partial_{M} V_{N}-\partial_{N} V_{M}-i\left[V_{M}, V_{N}\right], V_{M}=t^{a} V_{M}^{a},\left(t^{a}=\sigma^{a} / 2\right.$, with $\sigma^{a}$ being Pauli matrices). In the axial-like gauge $V_{z}=0$, the vector field $V_{\mu}^{a}(x, z=0)$ corresponds to the source for the vector current $J_{\mu}^{a}(x)$. To obtain the equations of motion for the transverse component of the field, it is convenient to work with the Fourier transform $\tilde{V}_{\mu}^{a}(p, z)$ of $V_{\mu}^{a}(x, z)$, for which one has

$$
\left(\partial_{z}\left[\frac{1}{z} e^{-z^{2}} \partial_{z} \tilde{V}_{\mu}^{a}(p, z)\right]+p^{2} \frac{1}{z} e^{-z^{2}} \tilde{V}_{\mu}^{a}(p, z)\right)_{\perp}=0 .
$$

(Here, and in the rest of this chapter, we find it convenient to follow the convention of Ref. [32], in which the oscillator scale $\kappa$ is treated as 1, i.e., we write below $z^{2}$ 
instead of $\kappa^{2} z^{2}, e^{-z^{2}}$ instead of $e^{-z^{2} \kappa^{2}}$, etc. Using dimensional analysis, the reader can easily restore the hidden factors of $\kappa$ in our expressions. In some cases, when $\kappa$ is not accompanied by $z$, we restore $\kappa$ explicitly.) The eigenvalue equation for wave functions $\psi_{n}(z)$ of the normalizable modes can be obtained from Eq. (3.3) by requiring $p^{2}=M_{n}^{2}$, which gives

$$
\partial_{z}\left[\frac{1}{z} e^{-z^{2}} \partial_{z} \psi_{n}\right]+M_{n}^{2} \frac{1}{z} e^{-z^{2}} \psi_{n}=0 .
$$

As noted in Ref. [32], the substitution

$$
\psi_{n}(z)=e^{z^{2} / 2} \sqrt{z} \Psi_{n}(z)
$$

gives a Schrödinger equation

$$
-\Psi_{n}^{\prime \prime}+\left(z^{2}+\frac{3}{4 z^{2}}\right) \Psi_{n}=M_{n}^{2} \Psi_{n},
$$

which happens to be exactly solvable. The resulting spectrum is $M_{n}^{2}=4(n+1)$ (with $n=0,1, \ldots)$, and the solutions $\psi_{n}(z)$ of the original equation (3.4) are given by

$$
\psi_{n}(z)=z^{2} \sqrt{\frac{2}{n+1}} L_{n}^{1}\left(z^{2}\right),
$$

where $L_{n}^{1}\left(z^{2}\right)$ are Laguerre polynomials. The functions $\psi_{n}(z)$ are normalized according to

$$
\int_{0}^{\infty} \frac{d z}{z} e^{-z^{2}} \psi_{m}(z) \psi_{n}(z)=\delta_{m n} .
$$

Correspondingly, the $\Psi_{n}(z)$ functions of the Schrödinger equation (3.6) are normalized by

$$
\int_{0}^{\infty} d z \Psi_{m}(z) \Psi_{n}(z)=\delta_{m n}
$$

i.e., just like wave functions of bound states in quantum mechanics. Note, however, that the functions $\Psi_{n}(z)$ behave like $z^{3 / 2}$ for small $z$, while quantum-mechanical wave functions of bound states with zero angular momentum have finite non-zero values at the origin.

\subsection{Bulk-to-Boundary Propagator}

It is convenient to represent $\tilde{V}_{\mu}^{a}(p, z)$ as the product of the 4-dimensional boundary field $\tilde{V}_{\mu}^{a}(p)$ and the bulk-to-boundary propagator $\mathcal{V}(p, z)$ which obeys the basic equation

$$
\partial_{z}\left[\frac{1}{z} e^{-z^{2}} \partial_{z} \mathcal{V}\right]+p^{2} \frac{1}{z} e^{-z^{2}} \mathcal{V}=0
$$


that follows from Eq. (3.3) and satisfies the boundary condition

$$
\mathcal{V}(p, z=0)=1 \text {. }
$$

Its general solution is given by the confluent hypergeometric functions of the first and second kind

$$
\mathcal{V}(p, z)=A z^{2}{ }_{1} F_{1}\left(a+1,2, z^{2}\right)+B U\left(a, 0, z^{2}\right),
$$

where $a=-p^{2} / 4 \kappa^{2}, A$ and $B$ are constants. Since the function proportional to $A$ is singular at $z=0$, we take $A=0$. Then, for $a>0$, the bulk-to-boundary propagator $\mathcal{V}(p, z)$ can be written as

$$
\mathcal{V}(p, z)=a \int_{0}^{1} d x x^{a-1} \exp \left[-\frac{x}{1-x} z^{2}\right] .
$$

It is easy to check that this expression satisfies Eqs. (3.10) and (3.11). Integrating by parts produces the representation

$$
\mathcal{V}(p, z)=z^{2} \int_{0}^{1} \frac{d x}{(1-x)^{2}} x^{a} \exp \left[-\frac{x}{1-x} z^{2}\right],
$$

from which it follows that if $p^{2}=0$ (or $\left.a=0\right)$, then

$$
\mathcal{V}(0, z)=1
$$

for all $z$. The integrand of Eq. (3.14) contains the generating function

$$
\frac{1}{(1-x)^{2}} \exp \left[-\frac{x}{1-x} z^{2}\right]=\sum_{n=0}^{\infty} L_{n}^{1}\left(z^{2}\right) x^{n}
$$

for the Laguerre polynomials $L_{n}^{1}\left(z^{2}\right)$, which gives the representation

$$
\mathcal{V}(p, z)=z^{2} \sum_{n=0}^{\infty} \frac{L_{n}^{1}\left(z^{2}\right)}{a+n+1}
$$

that can be analytically continued into the timelike $a<0$ region. One can see that $\mathcal{V}(p, z)$ has poles there at expected locations $p^{2}=4(n+1) \kappa^{2}$.

The same representation for $\mathcal{V}(p, z)$ can be obtained from the Green's function

$$
G\left(p ; z, z^{\prime}\right)=\sum_{n=0}^{\infty} \frac{\psi_{n}(z) \psi_{n}\left(z^{\prime}\right)}{p^{2}-M_{n}^{2}}
$$

corresponding to Eq. (3.10), namely,

$$
\begin{aligned}
\mathcal{V}\left(p, z^{\prime}\right) & =-\left[\frac{1}{z} e^{-z^{2}} \partial_{z} G\left(p ; z, z^{\prime}\right)\right]_{z=\epsilon \rightarrow 0} \\
& =-\sum_{n=0}^{\infty} \frac{\sqrt{8(n+1)} \psi_{n}\left(z^{\prime}\right)}{p^{2}-M_{n}^{2}}=-4 \sum_{n=0}^{\infty} \frac{z^{\prime 2} L_{n}^{1}\left(z^{\prime 2}\right)}{p^{2}-M_{n}^{2}}
\end{aligned}
$$


which coincides with Eq. (3.17).

The two-point density function can also be obtained from the Green's function:

$$
\begin{aligned}
\Sigma\left(p^{2}\right) & =\frac{1}{g_{5}^{2}}\left[\frac{1}{z^{\prime}} e^{-z^{\prime 2}} \partial_{z^{\prime}}\left[\frac{1}{z} e^{-z^{2}} \partial_{z} G\left(p ; z, z^{\prime}\right)\right]\right]_{z, z^{\prime}=\epsilon \rightarrow 0} \\
& =\sum_{n=0}^{\infty} \frac{f_{n}^{2}}{p^{2}-M_{n}^{2}}
\end{aligned}
$$

where the coupling constants $f_{n}=\kappa^{2} \sqrt{8(n+1)} / g_{5}$ obtained in [32] are determined by

$$
f_{n}=\left.\frac{1}{g_{5} z} e^{-z^{2}} \partial_{z} \psi_{n}(z)\right|_{z=\epsilon \rightarrow 0} .
$$

The propagator $\mathcal{V}(p, z)$ can be represented now as

$$
\mathcal{V}(p, z)=g_{5} \sum_{n=0}^{\infty} \frac{f_{n} \psi_{n}(z)}{M_{n}^{2}-p^{2}}
$$

where $\psi_{n}(z)$ are the original wave functions (3.7) corresponding to the solutions of the eigenvalue equation (3.4).

Given the structure of Eq. (3.21), it is natural to introduce the conjugate wave functions

$$
\begin{aligned}
\phi_{n}(z) & \equiv \frac{1}{M_{n} z} e^{-z^{2}} \partial_{z} \psi_{n}(z) \\
& =\frac{\sqrt{2}}{n+1} e^{-z^{2}}\left[L_{n}^{1}\left(z^{2}\right)-z^{2} L_{n-1}^{2}\left(z^{2}\right)\right] \\
& =\sqrt{2} e^{-z^{2}} L_{n}\left(z^{2}\right)
\end{aligned}
$$

whose nonzero values at the origin $f_{n} g_{5} / M_{n}$ are proportional to the coupling constant $f_{n}$ (in this particular case, $f_{n} g_{5} / M_{n}=\sqrt{2} \kappa$ ). The inverse relation between the $\psi$ and $\phi$ wave functions

$$
\psi_{n}(z)=-\frac{z}{M_{n}} e^{z^{2}} \partial_{z} \phi_{n}(z)
$$

can be obtained from Eq. (3.4). The $\phi$-functions are normalized by

$$
\int_{0}^{\infty} d z z e^{z^{2}} \phi_{m}(z) \phi_{n}(z)=\delta_{m n} .
$$

In particular, for the lowest states, we have

$$
\phi_{0}(z)=\sqrt{2} e^{-z^{2}} \quad, \quad \phi_{1}(z)=\sqrt{2} e^{-z^{2}}\left(1-z^{2}\right) .
$$


Just like zero angular momentum oscillator wave functions in quantum mechanics,

these functions have finite values at $z=0$. They also have a Gaussian fall-off $e^{-z^{2}}$ for large $z$. To make a more close analogy with the oscillator wave functions, it makes sense to absorb the weight $e^{z^{2}}$ in Eq. (3.25) into the wave functions, i.e., to introduce " $\Phi$ " wave functions

$$
\Phi_{n}(z) \equiv e^{z^{2} / 2} \phi_{n}(z)=\frac{1}{M_{n} z} e^{-z^{2} / 2} \partial_{z} \psi_{n}(z)=\sqrt{2} L_{n}\left(z^{2}\right),
$$

which are nonzero at $z=0$, decrease like $e^{-z^{2} / 2}$ for large $z$, and are normalized according to

$$
\int_{0}^{\infty} d z z \Phi_{m}(z) \Phi_{n}(z)=\delta_{m n} .
$$

The presence of the $z$ weight in this condition (which cannot be absorbed into wave functions without spoiling their behavior at $z=0$ ) suggests that pursuing the analogy with quantum mechanics one should treat $z$ as the radial variable of a 2-dimensional quantum mechanical system.

\subsection{Three-Point Function}

The variation of the trilinear (in $V$ ) term of the action

$$
S_{\text {AdS }}^{(3)}=-\frac{\epsilon_{a b c}}{2 g_{5}^{2}} \int d^{4} x \int_{\epsilon}^{\infty} \frac{d z}{z} e^{-z^{2}}\left(\partial_{\mu} V_{\nu}^{a}\right) V^{\mu, b} V^{\nu, c}
$$

calculated on the solutions of the basic equation (3.10) gives the following result for the 3-point correlator:

$$
\begin{aligned}
\left\langle J_{a}^{\alpha}\left(p_{1}\right) J_{b}^{\beta}\left(-p_{2}\right) J_{c}^{\mu}(q)\right\rangle & =\epsilon_{a b c}(2 \pi)^{4} \frac{2 i}{g_{5}^{2}} \delta^{(4)}\left(p_{1}-p_{2}+q\right) \\
& \times T^{\alpha \beta \mu}\left(p_{1}, p_{2}, q\right) W\left(p_{1}, p_{2}, q\right)
\end{aligned}
$$

with the dynamical part given by

$$
W\left(p_{1}, p_{2}, q\right) \equiv \int_{\epsilon}^{\infty} \frac{d z}{z} e^{-z^{2}} \mathcal{V}\left(p_{1}, z\right) \mathcal{V}\left(p_{2}, z\right) \mathcal{V}(q, z),
$$

and the kinematical factor having the structure of a nonabelian three-field vertex:

$$
\begin{aligned}
T^{\alpha \beta \mu}\left(p_{1}, p_{2}, q\right) & =\eta^{\alpha \mu}\left(q-p_{1}\right)^{\beta}-\eta^{\beta \mu}\left(p_{2}+q\right)^{\alpha} \\
& +\eta^{\alpha \beta}\left(p_{1}+p_{2}\right)^{\mu} .
\end{aligned}
$$

Incorporating the representation Eq. (3.22) for the bulk-to-boundary propagators gives the expression

$$
T\left(p_{1}^{2}, p_{2}^{2}, Q^{2}\right)=\sum_{n, k=1}^{\infty} \frac{f_{n} f_{k} F_{n k}\left(Q^{2}\right)}{\left(p_{1}^{2}-M_{n}^{2}\right)\left(p_{2}^{2}-M_{k}^{2}\right)}
$$


for $T\left(p_{1}^{2}, p_{2}^{2}, Q^{2}\right) \equiv W\left(p_{1}, p_{2}, q\right) / g_{5}^{2}$ as a sum over the poles of the bound states in the initial and final states. In the $z$-integral of Eq. (3.31), the contribution of each bound state is accompanied by its wave function $\psi_{n}(z)$, while the $q$-channel is represented by $\mathcal{J}(Q, z)=\mathcal{V}(i Q, z)$. This gives the $Q^{2}$-dependent coefficients

$$
F_{n k}\left(Q^{2}\right)=\int_{0}^{\infty} \frac{d z}{z} e^{-z^{2}} \mathcal{J}(Q, z) \psi_{n}(z) \psi_{k}(z)
$$

which have the meaning of transition form factors. Note that since $\mathcal{J}(0, z)=1$, the orthonormality relation (3.8) assures that $F_{n n}\left(Q^{2}=0\right)=1$ for diagonal transitions and $F_{n k}\left(Q^{2}=0\right)=0$ if $n \neq k$.

The factor $\mathcal{J}(Q, z)$ can be written as a sum of monopole contributions from the infinite tower of vector mesons:

$$
\mathcal{J}(Q, z)=g_{5} \sum_{m=1}^{\infty} \frac{f_{m} \psi_{m}(z)}{Q^{2}+M_{m}^{2}} .
$$

This decomposition, discussed in Refs. [46, 36], directly follows from Eq. (3.22). As a result, the form factors $F_{n k}\left(Q^{2}\right)$ can be written in the form of a generalized VMD representation:

$$
F_{n k}\left(Q^{2}\right)=\sum_{m=1}^{\infty} \frac{F_{m, n k}}{1+Q^{2} / M_{m}^{2}}
$$

where the coefficients $F_{m, n k}$ are given by the overlap integrals

$$
F_{m, n k}=\frac{g_{5} f_{m}}{M_{m}^{2}} \int_{0}^{\infty} \frac{d z}{z} e^{-z^{2}} \psi_{m}(z) \psi_{n}(z) \psi_{k}(z)
$$

\subsection{Form Factors}

In terms of the $\Psi$ wave functions of the Schrödinger equation (3.6), the form factors are given by

$$
F_{n k}\left(Q^{2}\right)=\int_{0}^{\infty} d z \mathcal{J}(Q, z) \Psi_{n}(z) \Psi_{k}(z)
$$

which looks like an expression for form factors in quantum mechanics. However, as we discussed above, the $\Psi$ wave functions are not direct analogues of quantum mechanical wave functions. For such an analogy, the $\Phi$ wave functions (3.27) are much more suitable objects. So, let us introduce form factors involving $\Phi$ wave functions

$$
\mathcal{F}_{n k}\left(Q^{2}\right) \equiv \int_{0}^{\infty} d z z \mathcal{J}(Q, z) \Phi_{n}(z) \Phi_{k}(z)
$$

Again, since $\mathcal{J}(Q=0, z)=1$ for all $z$, the normalization condition (3.28) for the $\Phi_{n}(z)$ wave functions guarantees that the diagonal form factors $\mathcal{F}_{n n}\left(Q^{2}\right)$ are normalized to 1 for $Q^{2}=0$, while the non-diagonal ones vanish when $Q^{2}=0$. To 
establish connection with $F_{n k}\left(Q^{2}\right)$ form factors, we use Eq. (3.27) to substitute $\Phi$ functions by derivatives of $\psi$ wave functions, which gives

$$
M_{n} M_{k} \mathcal{F}_{n k}\left(Q^{2}\right)=\int_{0}^{\infty} \frac{d z}{z} e^{-z^{2}} \mathcal{J}(Q, z) \psi_{n}^{\prime}(z) \psi_{k}^{\prime}(z)
$$

Integrating $\psi_{k}^{\prime}$ by parts, taking into account that $\psi_{k}(0)=0$ and incorporating the eigenvalue equation (3.4) for $\psi_{n}$ gives

$$
\begin{aligned}
M_{n} M_{k} \mathcal{F}_{n k}\left(Q^{2}\right) & =M_{n}^{2} F_{n k}\left(Q^{2}\right) \\
& -\int_{0}^{\infty} \frac{d z}{z} e^{-z^{2}} \psi_{n}^{\prime}(z) \psi_{k}(z) \partial_{z} \mathcal{J}(Q, z)
\end{aligned}
$$

Similarly, integrating $\psi_{n}^{\prime}$ by parts we obtain

$$
\begin{aligned}
M_{n} M_{k} \mathcal{F}_{n k}\left(Q^{2}\right) & =M_{k}^{2} F_{n k}\left(Q^{2}\right) \\
& -\int_{0}^{\infty} \frac{d z}{z} e^{-z^{2}} \psi_{n}(z) \psi_{k}^{\prime}(z) \partial_{z} \mathcal{J}(Q, z) .
\end{aligned}
$$

Adding these two expressions, integrating $\left(\psi_{n} \psi_{k}\right)^{\prime}$ by parts and using the basic equation (3.10) for $\mathcal{J}(Q, z)$ gives

$$
F_{n k}\left(Q^{2}\right)=\frac{2 M_{n} M_{k}}{Q^{2}+M_{n}^{2}+M_{k}^{2}} \mathcal{F}_{n k}\left(Q^{2}\right)
$$

For the case of diagonal $n \rightarrow n$ transitions this gives

$$
F_{n n}\left(Q^{2}\right)=\frac{\mathcal{F}_{n n}\left(Q^{2}\right)}{1+Q^{2} / 2 M_{n}^{2}}
$$

expression similar to that derived in Ref. [36].

Thus, we can obtain $F_{n k}\left(Q^{2}\right)$ form factors from the basic form factors $\mathcal{F}_{n k}\left(Q^{2}\right)$. Note, that these form factors also have a generalized VMD representation

$$
\mathcal{F}_{n k}\left(Q^{2}\right)=\sum_{m=1}^{\infty} \frac{\mathcal{F}_{m, n k}}{1+Q^{2} / M_{m}^{2}},
$$

with the coefficients $\mathcal{F}_{m, n k}$ given by the overlap integrals

$$
\begin{aligned}
\mathcal{F}_{m, n k} & =\frac{g_{5} f_{m}}{M_{m}^{2}} \int_{0}^{\infty} d z z \psi_{m}(z) \Phi_{n}(z) \Phi_{k}(z) \\
& =\frac{1}{m+1} \int_{0}^{\infty} d x x L_{m}^{1}(x) L_{n}(x) L_{k}(x) .
\end{aligned}
$$

For the lowest diagonal transition (i.e., for $n=k=0$ ) we have

$$
\mathcal{F}_{00}\left(Q^{2}\right)=2 \int_{0}^{\infty} d z z e^{-z^{2}} \mathcal{J}(Q, z) .
$$


Incorporating the representation (3.14) for $\mathcal{J}(Q, z)$ and using $a=Q^{2} / 4 \kappa^{2}$, we obtain

$$
\mathcal{F}_{00}\left(Q^{2}\right)=\frac{1}{1+a}=\frac{1}{1+Q^{2} / M_{0}^{2}} .
$$

Here, we took into account that the mass of the lowest bound state (i.e., $\rho$-meson) is $M_{0}=M_{\rho}=2 \kappa$.

Notice, that we obtained exact vector meson dominance for $\mathcal{F}_{00}\left(Q^{2}\right)$ : this form factor is completely determined by the lowest bound state in the $q$-channel. The higher states do not contribute because the overlap integral $\mathcal{F}_{m, 00}$ corresponding to the contribution of the $m^{\text {th }} q$-channel bound state vanishes for $m>0$ :

$$
\mathcal{F}_{m, 00}=2 \int_{0}^{\infty} d z z^{3} e^{-z^{2}} L_{m}^{1}\left(z^{2}\right)=\delta_{m 0} .
$$

In the case of the $F_{00}\left(Q^{2}\right)$ form factor, we have

$$
\begin{aligned}
F_{00}\left(Q^{2}\right) & =\frac{1}{(1+a)(1+a / 2)} \\
& =\frac{2}{1+Q^{2} / M_{0}^{2}}-\frac{1}{1+Q^{2} / M_{1}^{2}} .
\end{aligned}
$$

Thus, the $F_{00}\left(Q^{2}\right)$ form factor is given by contributions from the lowest two $q$-channel bound states. Since $F_{00}\left(Q^{2}\right) \sim 1 / Q^{4}$ for large $Q^{2}$, exact VMD is impossible for this form factor: other resonances are needed to "conspire" to cancel their leading $1 / Q^{2}$ terms at large $Q^{2}$. In the soft-wall model, this cancellation is provided by just the first excited state.

For small $Q^{2}$, the form factor $F_{00}^{\mathrm{S}}\left(Q^{2}\right)$ has the following expansion:

$$
F_{00}\left(Q^{2}\right)=\left[1-\frac{3}{2} \frac{Q^{2}}{M_{0}^{2}}+\frac{7}{4} \frac{Q^{4}}{M_{0}^{4}}+\mathcal{O}\left(Q^{6}\right)\right] .
$$

The Lorentz structure of the 3-point function in the soft-wall model is the same as in the hard-wall model considered in Ref. [36], where it was shown that electric $G_{C}$, magnetic $G_{M}$ and quadrupole $G_{Q}$ form factors (for definitions, see, e.g., [42, 36]) of the $n^{\text {th }}$ bound state are all expressed through the $F_{n n}\left(Q^{2}\right)$ form factor:

$$
\begin{aligned}
& G_{Q}^{(n)}\left(Q^{2}\right)=-F_{n n}\left(Q^{2}\right), \quad G_{M}^{(n)}\left(Q^{2}\right)=2 F_{n n}\left(Q^{2}\right), \\
& G_{C}^{(n)}\left(Q^{2}\right)=\left(1-\frac{Q^{2}}{6 M^{2}}\right) F_{n n}\left(Q^{2}\right) .
\end{aligned}
$$

The same relations hold for the soft-wall model. As a result, small- $Q^{2}$ expansion of the electric form factor of the lowest bound state in the soft-wall model is given by

$$
\begin{aligned}
G_{00}\left(Q^{2}\right) & =\left[1-\frac{Q^{2}}{6 M_{0}^{2}}\right] F_{00}\left(Q^{2}\right) \\
& =\left[1-\frac{5}{3} \frac{Q^{2}}{M_{0}^{2}}+2 \frac{Q^{4}}{M_{0}^{4}}+\mathcal{O}\left(Q^{6}\right)\right]
\end{aligned}
$$


and the electric radius for the $\rho$-meson in the soft-wall model is

$$
\left\langle r_{\rho}^{2}\right\rangle^{\mathrm{S}}=0.655 \mathrm{fm}^{2} \text {. }
$$

This radius is larger than the value $\left\langle r_{\rho}^{2}\right\rangle^{\mathrm{H}}=0.53 \mathrm{fm}^{2}$ that we obtained in Ref. [36] in the case of the hard-wall cutoff.

The radius of the $n^{\text {th }}$ excited state can be found from the slope of $F_{n n}\left(Q^{2}\right)$. The latter can be calculated using Eqs. (3.7), (3.34). Defining the slope coefficient $S_{n}$ by

$$
\left.\frac{d}{d Q^{2}} F_{n n}\left(Q^{2}\right)\right|_{Q^{2}=0} \equiv-\frac{S_{n}}{M_{0}^{2}}
$$

and using explicit form of Laguerre polynomials, we find

$$
S_{n}=\sum_{m, l=0}^{n} C_{n+1}^{m+1} C_{n+1}^{l+1}(-1)^{l+m} \frac{(m+l+1) !}{(n+1) m ! l !} \sum_{p=1}^{m+l+2} \frac{1}{p}
$$

$\left(C_{\alpha}^{\beta}\right.$ are binomial coefficients). A faster algorithm for numerical calculations is provided by the formula

$$
S_{n}=\sum_{m=0}^{n} C_{n}^{m} C_{m+n+1}^{n} \sum_{k=0}^{n-m} C_{n-m}^{k}(-2)^{k} \sum_{p=1}^{2 m+k+2} \frac{1}{p} .
$$

For $n=0$, these expressions give the result $S_{0}=3 / 2$ corresponding to Eq. (3.51). For higher states, we have $S_{1}=23 / 12, S_{2}=11 / 5, S_{3} \approx 2.415, S_{10} \approx 3.245$, $S_{20} \approx 3.816, S_{50} \approx 4.633, S_{100} \approx 5.281, S_{150} \approx 5.667, S_{200} \approx 5.943$. For $n \geq 2$, these values are well approximated by a simple empirical formula

$$
S_{n} \approx \ln (n+1)+\frac{2}{3}+\frac{5}{4(n+1)} .
$$

Thus, the squared sizes of excited states increase with the excitation number $n$. However, contrary to expectations of Ref. [32], the raise is only logarithmic, $\left\langle r_{n}^{2}\right\rangle^{\mathrm{S}} \sim \ln n$ rather than linear. Such an outcome is not unnatural since Eq. (3.56) differs from the identity

$$
\sum_{m, l=0}^{n} C_{n+1}^{m+1} C_{n+1}^{l+1}(-1)^{l+m} \frac{(m+l+1) !}{(n+1) m ! l !}=1
$$

(that follows from the normalization condition (3.9)) by the sum

$$
\left.\sum_{p=1}^{m+l+2} \frac{1}{p}\right|_{m+l \rightarrow \infty} \sim \ln (m+l+2)
$$

which has a logarithmic behavior for large $m+l$, and for large $n$ it may be approximated by $\ln n$ for the bulk of $m, l$ values. However, it would be interesting to derive a formal proof.

It should be noted, that in the hard-wall model, the slope of $F_{n n}\left(Q^{2}\right)$ at $Q^{2}=0$ decreases with $n$. For the lowest state, the value $S_{1}^{H}=1.192$ was found in Ref. [36]. For higher radial excitations, we have $S_{2}^{H}=0.877, S_{3}^{H}=0.833, S_{10}^{H}=0.806$, $S_{20}^{H}=0.804, S_{100}^{H}=0.803$, i.e., $\left\langle r_{n}^{2}\right\rangle^{H}$ tends to a constant value as $n \rightarrow \infty$. 


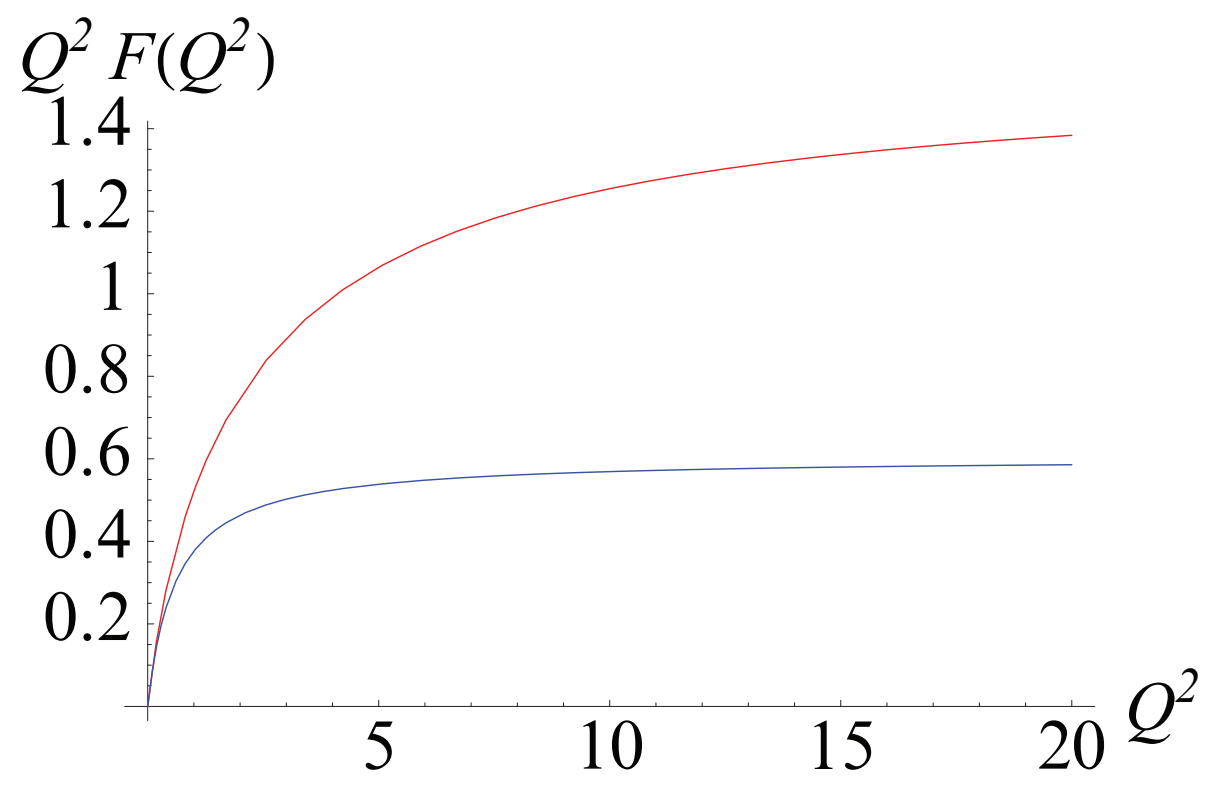

Figure 3.1: $Q^{2}$-multiplied $\rho$-meson form factor $\mathcal{F}_{00}\left(Q^{2}\right)$ (displayed in $\mathrm{GeV}^{2}$ ) as a function of $Q^{2}$ (given in $\mathrm{GeV}^{2}$ ) in hard-wall (upper line, red online) and soft-wall (lower line, blue online) models.

\subsection{Comparison with Hard-Wall Model}

Note that in the hard-wall model considered in Ref. [36], all the $q$-channel states give nonzero contributions to $\mathcal{F}_{00}\left(Q^{2}\right)$. In fact, it is strongly dominated by two lowest $q$-channel states. The role of the first excitation in the hard-wall model is especially important for large $Q^{2}$ : it gives asymptotically $2.061 M_{\rho}^{2} / Q^{2}$ while the lowest state contributes only $0.619 M_{\rho}^{2} / Q^{2}$.

It should also be mentioned that in both models $\mathcal{F}_{00}\left(Q^{2}\right)$ has $\sim 1 / Q^{2}$ behavior for large $Q^{2}$. However, the normalization of the asymptotic behavior in hard-wall model is much larger than in soft-wall model: $\mathcal{F}_{00}^{H}\left(Q^{2}\right) \rightarrow 2.566 M_{\rho}^{2} / Q^{2}$, while $\mathcal{F}_{00}^{\mathrm{S}}\left(Q^{2}\right) \rightarrow$ $M_{\rho}^{2} / Q^{2}$.

As discussed in Refs. [35, 36], to calculate the large- $Q^{2}$ behavior of $\mathcal{F}_{00}^{H}\left(Q^{2}\right)$, one should take the large- $Q^{2}$ limit of $\mathcal{J}^{\mathrm{H}}(Q, z)$, which is given by $z Q K_{1}(z Q) \equiv \mathcal{K}(Q z)$, the free-field version of the nonnormalizable mode. Asymptotically, it behaves like $e^{-Q z}$, so only small values of $z$ are important in the relevant integral. As a result,

$$
\mathcal{F}_{00}^{H}\left(Q^{2}\right) \rightarrow \frac{\left|\Phi_{0}^{\mathrm{H}}(0)\right|^{2}}{Q^{2}} \int_{0}^{\infty} d \chi \chi^{2} K_{1}(\chi)=\frac{2\left|\Phi_{0}^{\mathrm{H}}(0)\right|^{2}}{Q^{2}}
$$

i.e., the large- $Q^{2}$ behavior of $\mathcal{F}_{00}^{\mathrm{H}}\left(Q^{2}\right)$ is determined by the value of the $\Phi$ wave function at the origin, which is given by

$$
\Phi_{0}^{\mathrm{H}}(0)=\frac{\sqrt{2} M_{\rho}}{\gamma_{0,1} J_{1}\left(\gamma_{0,1}\right)} \approx 1.133 M_{\rho} .
$$


The nonnormalizable mode $\mathcal{J}^{\mathrm{S}}(Q, z)$ of the soft-wall model should also convert into $\mathcal{K}(Q z)$ when $Q^{2}$ is large. To see this directly, we compare the integral representation

$$
\mathcal{K}(Q z)=z^{2} \int_{0}^{1} \frac{d x}{(1-x)^{2}} \exp \left[-\frac{(1-x) Q^{2}}{4 x}-\frac{x z^{2}}{1-x}\right]
$$

for $\mathcal{K}(Q z)$ and the representation

$$
\mathcal{J}^{\mathrm{S}}(Q, z)=z^{2} \int_{0}^{1} \frac{d x}{(1-x)^{2}} \exp \left[-\frac{Q^{2}}{4} \ln \left(\frac{1}{x}\right)-\frac{x z^{2}}{1-x}\right]
$$

for $\mathcal{J}^{\mathrm{S}}(Q, z)$ following from Eq. (3.14). For large $Q^{2}$, both integrals are dominated by the region where $1-x \sim 2 z \kappa^{2} / Q$. Then both $(1-x) / x$ and $\ln (1 / x)$ may be approximated by $(1-x)$. Thus, large- $Q^{2}$ behavior of $\mathcal{J}^{\mathrm{S}}(Q, z)$ coincides with that of $\mathcal{K}(Q z)$, and Eq. (3.61) is applicable in soft-wall model as well, with the normalization of the asymptotically leading term determined by the value of $\Phi_{0}^{\mathrm{S}}(z)$ at the origin, which is

$$
\Phi_{0}^{\mathrm{S}}(0)=M_{\rho} / \sqrt{2} \approx 0.707 M_{\rho} .
$$

Hence, it is the difference in the values of $\Phi$ wave functions at the origin that explains the difference in the asymptotic normalization of $\mathcal{F}_{00}\left(Q^{2}\right)$ in these two models.

The difference in the values of $\Phi(0)$ leads also to difference in the values of coupling constants $f_{n}$ related to $\Phi_{n}(0)$ by

$$
f_{n}=\Phi_{n}(0) M_{n} / g_{5} .
$$

The constant $g_{5}$ is determined by matching the asymptotic behavior

$$
\Sigma^{\operatorname{AdS}}\left(p^{2}\right) \rightarrow-\frac{p^{2}}{2 g_{5}^{2}} \ln \left(p^{2}\right)
$$

of the two-point function $\Sigma^{\mathrm{AdS}}\left(p^{2}\right)$ given by Eq. (3.20) with the QCD result for the correlator of the vector currents $J_{\mu}=\bar{d} \gamma_{\mu} u$ having quantum numbers of the $\rho^{+}$ meson. Since

$$
\Sigma^{\mathrm{QCD}}\left(p^{2}\right) \rightarrow-\frac{N_{c}}{12 \pi^{2}} p^{2} \ln \left(p^{2}\right),
$$

we have

$$
g_{5}=\sqrt{2} \pi
$$

for $N_{c}=3$. This gives

$$
f_{\rho}^{\mathrm{S}}=\frac{M_{\rho}^{2}}{2 \pi} \approx(309 \mathrm{MeV})^{2}
$$

for the $\rho$ coupling constant in the soft-wall model, and

$$
f_{\rho}^{\mathrm{H}}=\frac{M_{\rho}^{2}}{\pi \gamma_{0,1} J_{1}\left(\gamma_{0,1}\right)} \approx(392 \mathrm{MeV})^{2}
$$


in the hard-wall model ${ }^{\dagger}$. The experimental value [22]

$$
f_{\rho}^{\exp }=(401 \pm 4 \mathrm{MeV})^{2}
$$

is very close to the hard-wall model result, and in this respect the hard-wall model is more successful. It may be also noted that, unlike the value $\left\langle r_{\rho}^{2}\right\rangle^{\mathrm{S}}=0.655 \mathrm{fm}^{2}$ in Eq. (4.5), the hard-wall model result $\left\langle r_{\rho}^{2}\right\rangle^{\mathrm{H}}=0.53 \mathrm{fm}^{2}$ for the $\rho$-meson charge radius obtained in our paper [36] practically coincides both with the Dyson-Schwinger model result of Ref.[43] and lattice gauge calculation reported in Ref. [44].

It is also instructive to consider the modified coupling $g_{\rho} \equiv f_{\rho} / M_{\rho}$ that has the dimension of mass, and determines the asymptotical behavior of the form factor. Its value in the soft-wall model

$$
g_{\rho}^{\mathrm{S}}=\frac{M_{\rho}}{2 \pi} \approx 123 \mathrm{MeV}
$$

is close to the experimental value of the pion decay constant $f_{\pi} \approx 131 \mathrm{MeV}$. Moreover, the pure $\rho$-pole result (4.65) is close to the experimental data on the pion form factor. So, it is tempting to take for the pion the same wave functions that were obtained in the $\rho$-meson case and use Eq. (4.65) as a model for the pion form factor. This was done in the paper [49] (that appeared after we submitted the original version [37] of the present paper to the arxive). Taking $\kappa=375 \mathrm{MeV}$ (which is slightly smaller than $\left.m_{\rho} / 2\right)$, the authors obtained good agreement of the $1 /\left(1+Q^{2} / 4 \kappa^{2}\right)$ curve with the pion form factor data (though the value of $f_{\pi}^{2}$ is then about $30 \%$ below the experimental one). However, within the model of Refs. [26, 28, 32], which we follow here, the analysis of the axial-vector current channel requires the inclusion of chiral symmetry breaking effects absent in the vector current channel. As a result, wave function equations for the pion are completely different from those for the $\rho$-meson. We discuss the pion form factor in a separate publication [38].

\subsection{Summary}

In the present chapter, we studied wave functions and form factors of vector mesons within the framework of the soft-wall model [32] which produces a more realistic spectrum for higher excited mesons [45] than the hard-wall model of Refs. [26, 27, 28]. Our analysis uses the approach similar to that we developed in Ref. [36] in application to the hard-wall model.

An essential element of our study of the soft-wall model is the integral representation, which we found for the bulk-to-boundary propagator $\mathcal{V}(p, z)$. It allows to write $\mathcal{V}(p, z)$ as an explicit expansion over bound state poles. In this sense, it plays the same role as the Kneser-Sommerfeld expansion that we used in our study [36] of the hard-wall model.

\footnotetext{
†The hard-wall model result $F_{\rho}^{1 / 2} \approx 329 \mathrm{MeV}$ presented in Ref. [26] corresponds to the $\left(\bar{u} \gamma_{\mu} u-\bar{d} \gamma_{\mu} d\right) / 2$ current which differs by $\sqrt{2}$ from the current $\left(\bar{u} \gamma_{\mu} u-\bar{d} \gamma_{\mu} d\right) / \sqrt{2}$ that has the same normalization as $\bar{d} \gamma_{\mu} u$.
} 
The pole expansion of $\mathcal{V}(p, z)$ involves " $\psi$ wave functions" that describe $z$ dependence of a particular pole contribution and are eigenfunctions of the 5D equation of motion. However, since $\psi_{n}(z)$ wave functions are not direct analogues of the usual quantum-mechanical wave functions, we introduced " $\Phi$ wave functions" resembling wave functions of oscillator states in quantum mechanics. In particular, the values of these functions at the origin give the couplings $g_{5} f_{n} / M_{n}$ of the bound states, and these functions exponentially decrease with $z^{2}$.

Analyzing the three-point function, we obtained expressions for transition form factors both in terms of the $\psi$ wave functions and the "more physical" $\Phi$ wave functions. We demonstrated that, just like in the hard-wall model, the form factors can be written in the form of generalized vector meson dominance representation, i.e., as a sum over all bound states in the channel of electromagnetic current (this result confirms the claim [46] that generalized VMD is a common feature of AdS/QCD models).

We derived an explicit expression for $\rho$-meson form factors, and analyzed their behavior in the regions of small and large $Q^{2}$. In particular, we calculated the $\rho$ meson electric radius in the soft-wall model, and found that it is larger than in the hard-wall model (the latter agrees with calculations in Dyson-Schwinger model [43] and lattice QCD [44]). Our calculation also demonstrated that the squared radii of higher excited states increase with $n$, the number of the radially excited level. However, contrary to expectations of Ref. [32], the increase is only logarithmic rather than linear. Another result is that, in the soft-wall model, the $\rho$-meson form factor $\mathcal{F}_{\rho}\left(Q^{2}\right)$ (corresponding to the overlap of the $\Phi$ wave functions) exhibits an exact VMD pattern, i.e., it is given by a single monopole term due to the lowest bound state in the $Q^{2}$-channel. In the case of the $\rho$-meson form factor $F_{\rho}\left(Q^{2}\right)$ (that is given by the overlap of the $\psi$-wave functions), we found a two-resonance dominance pattern, where just two lowest bound states in the $Q^{2}$-channel contribute.

Analyzing the large- $Q^{2}$ behavior of the $\mathcal{F}_{\rho}\left(Q^{2}\right)$ form factor (given by exact $\rho$-pole VMD), we established that its asymptotic normalization in the soft-wall model is much lower (by factor 2.566) than that of the hard-wall model. This difference is explained by essentially lower value of the soft-wall model $\Phi$ wave function at the origin.

Finally, we calculated the $\rho$-meson coupling constant $f_{\rho}$ both in the soft-wall and hard-wall models, and found that the experimental value is closer to the hard-wall model result. 


\section{Chapter 4}

\section{Massless Pion in Holographic Model of QCD}

\subsection{Introduction}

In general, the vector sector is less sensitive to infrared (IR) effects, since global vector symmetry is not broken in QCD. However, the axial-vector sector appears to be very sensitive to the particular way the chiral symmetry is broken or, in other words, to the bulk content and the shape of the IR wall [32]. In this respect, one of the interesting objects to study in the holographic dual models of QCD is the pion. The properties of the pion have been studied in various holographic approaches, (see e.g. Refs. [25, 26, 28, 58, 104, 29, 105, 108, 111, 30, 35]). In particular, the approach of Ref. [26] (see also recent papers [105, 108, 111]) managed to reproduce the (Gell-Mann-Oakes-Renner) relation $m_{\pi}^{2} \sim m_{q}$ between the quark mass $m_{q}$ and mass of the pion $m_{\pi}$ and also the $g_{\rho \pi \pi}$ coupling (the coupling between $\rho$ meson and two pions). In Ref. [28], the solution of the pion wave-function equation was explicitly written for the $m_{q}=0$ limit.

In this chapter*, working in the framework of the hard-wall model, we describe a formalism to calculate the form factor and wave functions (and also the density function) of the pion (see also [59]). Since the fits of Ref. [26] give a very small $m_{q} \sim 2 \mathrm{MeV}$ value for the explicit chiral symmetry breaking parameter $m_{q}$, we consider only the chiral limit $m_{q}=0$ of the hard-wall holographic dual model of two-flavor QCD. Resorting to the chiral limit allows us to utilize one of the main advantages of AdS/QCD - the possibility to work with explicit analytic solutions of the basic equations of motion. Expressing the pion form factor in terms of these solutions, we are able, in particular, to extract and analyze the behavior of the pion electric radius in various regions of the holographic parameters space. On the numerical side, we come to the conclusion that the radius of the pion is smaller than what is known from experiment. However, we suggest that, as in case of the radius of the $\rho$ meson, smoothing the IR wall may increase the pion radius.

In our analysis, we introduce and systematically use two types of holographic

*The main results from the Ref. [38] are printed by permission from the APS, see Appendix. 
wave functions $\Phi(z)$ and $\Psi(z)$, which are conjugate to each other and basically similar to the analogous objects introduced in our papers [36, 37], where we studied vector mesons.

We start with recalling the basics of the hard-wall model in the axial sector and some results obtained in Ref. [26], in particular, the form of the relevant action, the eigenvalue equations for bound states and their solutions. Then, we describe a formalism for calculating the pion form factor and express it in terms of the two wave functions mentioned above. We also discuss the relation of our AdS/QCD results to experimental data. Furthermore, we express the values of the pion decay constant and the pion charge radius in terms of the fundamental parameters of the theory and study their behavior in different regions of the parametric space. At the end, we study the behavior of the pion form factor at large momentum transfer. Finally, we summarize the chapter.

\subsection{Preliminaries}

In the holographic model of hadrons, QCD resonances correspond to Kaluza-Klein $(\mathrm{KK})$ excitations in the sliced $\mathrm{AdS}_{5}$ background. In particular, vector mesons correspond to the KK modes of the transverse vector gauge field in this background. Since the gauge symmetry in the vector sector of the hard-wall model is not broken, the longitudinal component of the vector gauge field is unphysical, and only transverse components correspond to physical mesons. Similarly, the axial-vector mesons are the modes of the transverse part of the axial-vector gauge field. However, because the axial-vector gauge symmetry is broken in the 5D background, the longitudinal components have physical meaning and are related to the pion field. This should be taken into account if we want to treat the pion in a consistent way.

\subsubsection{Action and Equations of Motion}

The standard prescription of the holographic model is that there is a correspondence between the $4 \mathrm{D}$ vector and axial-vector currents and the corresponding 5D gauge fields:

$$
\begin{aligned}
& J_{V \mu}^{a}(x)=\bar{q}(x) \gamma_{\mu} t^{a} q(x) \rightarrow V_{\mu}^{a}(x, z) \\
& J_{A \mu}^{a}(x)=\bar{q}(x) \gamma_{\mu} \gamma_{5} t^{a} q(x) \rightarrow A_{\mu}^{a}(x, z),
\end{aligned}
$$

where $t^{a}=\sigma^{a} / 2,\left(a=1,2,3\right.$ and $\sigma^{a}$ are usual Pauli matrices $)$.

In general, one can write $A=A_{\perp}+A_{\|}$, where $A_{\perp}$ and $A_{\|}$are transverse and longitudinal components of the axial-vector field. The spontaneous symmetry breaking causes $A_{\|}$to be physical and associated with the Goldstone boson, the pion in this case. The longitudinal component may be written in the form: $A_{M \|}^{a}(x, z)=\partial_{M} \psi^{a}(x, z)$. Then $\psi^{a}(x, z)$ corresponds to the pion field. Physics of the axial-vector and pseudoscalar sectors is described by the action

$$
S_{\mathrm{AdS}}^{A}=\operatorname{Tr} \int d^{4} x d z\left[\frac{1}{z^{3}}\left(D^{M} X\right)^{\dagger}\left(D_{M} X\right)+\frac{3}{z^{5}} X^{\dagger} X-\frac{1}{4 g_{5}^{2} z} A^{M N} A_{M N}\right]
$$


where $D X=\partial X-i A_{L} X+i X A_{R},\left(A_{L(R)}=V \pm A\right)$ and $X(x, z)=v(z) U(x, z) / 2$ is taken as a product of the chiral field $U(x, z)=\exp \left(2 i t^{a} \pi^{a}(x, z)\right)$ and the function $v(z)=m_{q} z+\sigma z^{3}$ containing the chiral symmetry breaking parameters $m_{q}$ and $\sigma$, with $m_{q}$ playing the role of the quark mass and $\sigma$ that of the quark condensate. Expanding $U(x, z)$ in powers of $\pi^{a}$ gives the relevant piece of the action

$$
S_{\mathrm{AdS}}^{A(2)}=\operatorname{Tr} \int d^{4} x d z\left[-\frac{1}{4 g_{5}^{2} z} A^{M N} A_{M N}+\frac{v^{2}(z)}{2 z^{3}}\left(A_{M}^{a}-\partial_{M} \pi^{a}\right)^{2}\right] .
$$

This Higgs-like mechanism breaks the axial-vector gauge symmetry by bringing a $z$-dependent mass term in the $A$-part of the lagrangian. Varying the action with respect to the transverse part of the axial-vector gauge field $A_{\perp \mu}^{a}(x, z)$ and representing the Fourier image of $A_{\perp \mu}^{a}(x, z)$ as $\tilde{A}_{\perp \mu}^{a}(p, z)$ we will get the following equation of motion

$$
\left[z^{3} \partial_{z}\left(\frac{1}{z} \partial_{z} \tilde{A}_{\mu}^{a}\right)+p^{2} z^{2} \tilde{A}_{\mu}^{a}-g_{5}^{2} v^{2} \tilde{A}_{\mu}^{a}\right]_{\perp}=0
$$

that determines physics of the axial-vector mesons, like $A_{1}$. The axial-vector bulk-toboundary propagator $\mathcal{A}(p, z)$ is introduced by the relation $\tilde{A}_{\perp \mu}^{a}(p, z)=\mathcal{A}(p, z) A_{\mu}^{a}(p)$. It satisfies Eq. (4.4) with boundary conditions (b.c.) $\mathcal{A}(p, 0)=1$ and $\mathcal{A}^{\prime}\left(p, z_{0}\right)=0$. Similarly, variation with respect to the longitudinal component $\partial_{\mu} \psi^{a}$ gives

$$
z^{3} \partial_{z}\left(\frac{1}{z} \partial_{z} \psi^{a}\right)-g_{5}^{2} v^{2}\left(\psi^{a}-\pi^{a}\right)=0
$$

Finally, varying with respect to $A_{z}$ produces

$$
p^{2} z^{2} \partial_{z} \psi^{a}-g_{5}^{2} v^{2} \partial_{z} \pi^{a}=0
$$

The pion wave function is determined from Eqs. (4.5) and (4.6) with b.c. $\partial_{z} \psi\left(z_{0}\right)=$ $0, \psi(\epsilon)=0$ and $\pi(\epsilon)=0$.

Within the framework of the model of Ref. [26], it is possible to derive the GellMann-Oakes-Renner relation $m_{\pi}^{2} \sim m_{q}$ producing massless pion in the $m_{q}=0$ limit. Taking $p^{2}=m_{\pi}^{2}$ in Eq. (4.6) gives

$$
\partial_{z} \pi=\frac{m_{\pi}^{2} z^{2}}{g_{5}^{2} v^{2}} \partial_{z} \psi
$$

A perturbative solution in the form of $m_{\pi}^{2}$ expansion was proposed in Ref [26], with $\psi(z)=\mathcal{A}(0, z)-1$ in the lowest order. Then it was shown that, in the $m_{q} \rightarrow 0$ limit, $\pi(z)$ tends to $-\theta\left(z-z_{0}\right)$ or, roughly speaking, $\pi=-1$ in this limit. Since our goal is to calculate the pion form factor in the chiral limit, this approximation will be sufficient for us. 


\subsubsection{Two-Point Function}

The spectrum in the axial-current channel consists of the pseudoscalar pion $\left\langle 0\left|J_{A}^{\alpha}\right| \pi(p)\right\rangle=$ $i f_{\pi} p^{\alpha}$ and axial-vector mesons $\left\langle 0\left|J_{A}^{\alpha}\right| A_{n}(p, s)\right\rangle=F_{A, n} \epsilon_{n}^{\alpha}(p, s)$, where $F_{A, n}$ corresponds to the $n^{\text {th }}$ axial-vector meson decay constant (and we ignored the flavor indexes). Thus, the two-point function for the axial-vector currents has the form:

$$
\left\langle J_{A}^{\alpha}(p) J_{A}^{\beta}(-p)\right\rangle=p^{\alpha} p^{\beta} \frac{f_{\pi}^{2}}{p^{2}}+\sum_{n} \Pi_{n}^{\alpha \beta}(p) \frac{F_{A, n}^{2}}{p^{2}-M_{A, n}^{2}} .
$$

where the meson polarization tensor is given by

$$
\Pi_{n}^{\alpha \beta}(p)=\sum_{s} \epsilon_{n}^{\alpha}(p, s) \epsilon_{n}^{\beta}(p, s)=-\eta^{\alpha \beta}+\frac{p^{\alpha} p^{\beta}}{M_{A, n}^{2}} .
$$

The representation for the two-point function can be also written as

$$
\left\langle J_{A}^{\alpha}(p) J_{A}^{\beta}(-p)\right\rangle=p^{\alpha} p^{\beta} \frac{f_{\pi}^{2}}{p^{2}}+\left(-\eta^{\alpha \beta}+\frac{p^{\alpha} p^{\beta}}{p^{2}}\right) \sum_{n} \frac{F_{A, n}^{2}}{p^{2}-M_{A, n}^{2}}+\text { (nonpole terms), }
$$

in which the second term on the rhs is explicitly transverse to $p$.

As noted in Ref. [26], using holographic correspondence one can relate the twopoint function to $\left[\partial_{z} A(p, z) / z\right]_{z=0}$ and derive that

$$
f_{\pi}^{2}=-\frac{1}{g_{5}^{2}}\left(\frac{1}{z} \partial_{z} A(0, z)\right)_{z=\epsilon \rightarrow 0}
$$

For large spacelike $p^{2}$, Eq. (4.4) gives the same solution as in case of vector mesons, and the same asymptotic logarithmic behavior, just as expected from QCD.

\subsubsection{Pion Wave Functions}

The longitudinal component of the axial-vector gauge field was defined as $A_{\|}=\partial \psi$. In the chiral limit, when $p^{2}=m_{\pi}^{2}=0$, we have $\partial_{z} \pi=0$, and the basic equation for $\psi$, Eq. (4.5) can be rewritten as the equation

$$
z^{3} \partial_{z}\left(\frac{1}{z} \partial_{z} \Psi\right)-g_{5}^{2} v^{2} \Psi=0
$$

for the function $\Psi \equiv \psi-\pi$. In the chiral limit, when $\pi(z) \rightarrow-1$, the value of $\Psi(\epsilon)$ tends to 1 as $\epsilon \rightarrow 0$. This value and the b.c. $\Psi^{\prime}\left(z_{0}\right)=0$ are the same as those for $\mathcal{A}(p, z)$ and, furthermore, Eq. (4.12) coincides with the $p^{2}=0$ version of equation (4.4) for $\mathcal{A}(p, z)$. Hence, the solution for $\Psi(z)$ coincides with $\mathcal{A}(0, z)$ :

$$
\Psi(z)=\mathcal{A}(0, z)
$$


and we may write

$$
f_{\pi}^{2}=-\frac{1}{g_{5}^{2}}\left(\frac{1}{z} \partial_{z} \Psi(z)\right)_{z=\epsilon \rightarrow 0}
$$

In our analysis of $\rho$-meson wave functions in Refs. [36, 37], we emphasized that it makes sense to consider also the conjugate functions $\Phi(z) \sim \Psi^{\prime}(z) / z$ of the corresponding Sturm-Liouville equation. As we observed, they are closer in their structure to the usual quantum mechanical bound state wave functions than the solutions of the original equation. In the pion case, it is convenient to define the $\Phi$ function as

$$
\Phi(z)=-\frac{1}{g_{5}^{2} f_{\pi}^{2}}\left(\frac{1}{z} \partial_{z} \Psi(z)\right)
$$

It vanishes at the IR boundary $z=z_{0}$ and, according to Eq. (4.11), is normalized as

$$
\Phi(0)=1
$$

at the origin. Note also that using Eq. (4.12) we can express $\Psi$ as derivative of $\Phi$ :

$$
\Psi(z)=-\frac{f_{\pi}^{2} z^{3}}{v^{2}} \partial_{z} \Phi(z)
$$

\subsection{Pion Electromagnetic Form Factor}

\subsubsection{Three-Point Function}

To obtain the pion form factor, we need to consider three-point correlation functions. The correlator should include the external EM current $J_{\mu}^{e l}(0)$ and currents having nonzero projection onto the pion states, e.g. the axial currents $J_{5 \alpha}^{a}\left(x_{1}\right), J_{5 \beta}^{a \dagger}\left(x_{2}\right)$

$$
\mathcal{T}_{\mu \alpha \beta}\left(p_{1}, p_{2}\right)=\int d^{4} x_{1} \int d^{4} x_{2} e^{i p_{1} x_{1}-i p_{2} x_{2}}\left\langle 0\left|\mathcal{T} J_{5 \beta}^{\dagger}\left(x_{2}\right) J_{\mu}^{\mathrm{el}}(0) J_{5 \alpha}\left(x_{1}\right)\right| 0\right\rangle,
$$

where $p_{1}, p_{2}$ are the corresponding momenta, with the momentum transfer carried by the EM source being $q=p_{2}-p_{1}$ (as usual, we denote $q^{2}=-Q^{2}, Q^{2}>0$ ). The spectral representation for the three-point function is a two-dimensional generalization of Eq. (4.8)

$\mathcal{T}^{\mu \alpha \beta}\left(p_{1}, p_{2}\right)=p_{1}^{\alpha} p_{2}^{\beta}\left(p_{1}+p_{2}\right)^{\mu} \frac{f_{\pi}^{2} F_{\pi}\left(Q^{2}\right)}{p_{1}^{2} p_{2}^{2}}+\sum_{n, m}($ transverse terms $)+($ nonpole terms $)$,

where the first term, longitudinal both with respect to $p_{1}^{\alpha}$ and $p_{2}^{\beta}$ contains the pion electromagnetic form factor $F_{\pi}\left(Q^{2}\right)$

$$
\left\langle\pi\left(p_{1}\right)\left|J_{\mu}^{e l}(0)\right| \pi\left(p_{2}\right)\right\rangle=F_{\pi}\left(q^{2}\right)\left(p_{1}+p_{2}\right)_{\mu},
$$


(normalized by $F_{\pi}(0)=1$ ), while other pole terms contain the contributions involving axial-vector mesons and are transverse either with respect to $p_{1}^{\alpha}$ or $p_{2}^{\beta}$, or both. Hence, the pion form factor can be extracted from the three-point function using

$$
\left.p_{1 \alpha} p_{2 \beta} \mathcal{T}^{\mu \alpha \beta}\left(p_{1}, p_{2}\right)\right|_{p_{1}^{2}=0, p_{2}^{2}=0}=\left(p_{1}+p_{2}\right)^{\mu} f_{\pi}^{2} F_{\pi}\left(Q^{2}\right) .
$$

\subsubsection{Trilinear Terms in $F^{2}$ Part of Action}

To obtain the form factor from the holographic model, we need the action of the third order in the fields. There are two types of terms contributing to the pion electromagnetic form factor: $|D X|^{2}$ term and $F^{2}$ terms. Let us consider first the contribution from $F^{2}$ terms. They contain $V V V, V A A$ and $A V A$ interactions and may be written as

$$
\left.S_{\mathrm{AdS}}^{F^{2}}\right|_{3}=\frac{i}{g_{5}^{2}} \operatorname{Tr} \int d^{4} x d z \frac{1}{z}\left(V_{M N}\left[V^{M}, V^{N}\right]+V_{M N}\left[A^{M}, A^{N}\right]+A_{M N}\left[V^{M}, A^{N}\right]\right),
$$

where $V_{M N}=\partial_{M} V_{N}-\partial_{N} V_{M}$ and $A_{M N}=\partial_{M} A_{N}-\partial_{N} A_{M}$. Taking $V_{z}=A_{z}=0$ gauge, we pick out the part of the action which is contributing to the 3-point function $\left\langle J_{5 \alpha} J_{\mu} J_{5 \beta}\right\rangle$ :

$$
W_{3}=\frac{i}{g_{5}^{2}} \operatorname{Tr} \int d^{4} x d z \frac{1}{z}\left(V_{\mu \nu}\left[A^{\mu}, A^{\nu}\right]+A_{\mu \nu}\left[V^{\mu}, A^{\nu}\right]\right) .
$$

Introducing Fourier transforms of fields, we define, as usual, $V_{\mu}(q, z)=\tilde{V}_{\mu}(q) \mathcal{V}(q, z)$ for the vector field, where $\tilde{V}_{\mu}(q)$ is the Fourier transform of the 4-dimensional field $V_{\mu}(x)$ and $\mathcal{V}(q, z)$ is the bulk-to-boundary propagator satisfying the equation

$$
z \partial_{z}\left(\frac{1}{z} \partial_{z} \mathcal{V}(q, z)\right)+q^{2} \mathcal{V}(q, z)=0
$$

with b.c. $\mathcal{V}(q, 0)=1$ and $\partial_{z} \mathcal{V}\left(q, z_{0}\right)=0$. It can be written as the sum

$$
\mathcal{V}(q, z)=g_{5} \sum_{m=1}^{\infty} \frac{f_{m} \psi_{m}^{V}(z)}{-q^{2}+M_{m}^{2}}
$$

involving all the bound states in the $q$-channel, with $M_{m}$ being the mass of the $m$ th bound state and $\psi_{m}^{V}(z)$ its wave function given by a solution of the basic equation of motion in the vector sector.

The projection (4.21) picks out only the longitudinal part $A_{\| \mu}(p, z)$ of the axialvector field. Taking into account that $A_{\| \mu}^{a}(x, z)=\partial_{\mu} \psi(x, z)$, we may write

$$
A_{\| \mu}^{a}(p, z)=i p_{\mu} \psi^{a}(p, z),
$$

where $A_{\| \mu}^{a}(p, z)$ and $\psi^{a}(p, z)$ are the Fourier transforms of $A_{\| \mu}^{a}(x, z)$ and $\psi(x, z)$, respectively. Furthermore, there is only one particle in the expansion over bound 
state in this case, namely, the massless pion. Thus, we have $A_{\| \mu}^{a}(p, z)=\tilde{A}_{\| \mu}^{a}(p) \psi(z)$ and, therefore,

$$
\psi^{a}(p, z)=-\frac{i p^{\alpha}}{p^{2}} \tilde{A}_{\| \alpha}^{a}(p) \psi(z)
$$

This allows us to rewrite $A_{\| \mu}^{a}(p, z)$ in the form

$$
A_{\| \mu}^{a}(p, z)=\frac{p^{\alpha} p_{\mu}}{p^{2}} \tilde{A}_{\| \alpha}^{a}(p) \psi(z)
$$

involving the longitudinal projector $p^{\alpha} p_{\mu} / p^{2}$ and the pion wave function $\psi(z)$, which is the solution of the basic equation (4.5). Using this representation and making Fourier transform of $W_{3}$ gives

$$
\begin{array}{r}
W_{3}=-\frac{1}{2 g_{5}^{2}} \epsilon_{a b c} \int \frac{d^{4} u d^{4} v d^{4} w}{(2 \pi)^{12}} i(2 \pi)^{4} \delta^{(4)}(u+v+w) \frac{u^{\mu} v^{\nu} u^{\alpha} v^{\beta}}{u^{2} v^{2}} \\
\tilde{A}_{\| \alpha}^{b}(u) \tilde{A}_{\| \beta}^{c}(v)\left[w_{\mu} \tilde{V}_{\nu}^{a}(w)-w_{\nu} \tilde{V}_{\mu}^{a}(w)\right] \int_{\epsilon}^{z_{0}} d z \frac{1}{z} \mathcal{V}(w, z) \psi^{2}(z)
\end{array}
$$

(notice that the second term in Eq.(4.23) vanishes for longitudinal axial-vector fields). Varying this functional with respect to sources produces the following 3point function:

$$
\begin{aligned}
\left\langle J_{V, a}^{\mu}(q) J_{\| A, b}^{\alpha}\left(p_{1}\right) J_{\| A, c}^{\beta}\left(-p_{2}\right)\right\rangle & =-i(2 \pi)^{4} \delta^{(4)}\left(q+p_{1}-p_{2}\right) \epsilon_{a b c} \frac{p_{1}^{\alpha} p_{2}^{\beta}}{p_{1}^{2} p_{2}^{2}}\left(p_{1}+p_{2}\right)^{\mu} \\
& \times \frac{1}{2 g_{5}^{2}} q^{2} \int_{\epsilon}^{z_{0}} d z \frac{1}{z} \mathcal{V}(q, z) \psi^{2}(z)
\end{aligned}
$$

where, anticipating the limit $p_{1}^{2} \rightarrow 0, p_{2}^{2} \rightarrow 0$, we took $\left(p_{1} q\right)=-\left(p_{2} q\right)=-q^{2} / 2$ in the numerator factors. Now, representing $\left\langle J_{V, a}^{\mu}(q) J_{\| A, b}^{\alpha}\left(p_{1}\right) J_{\| A, c}^{\beta}\left(-p_{2}\right)\right\rangle=i(2 \pi)^{4} \delta^{(4)}(q+$ $\left.p_{1}-p_{2}\right) \epsilon_{a b c} \mathcal{T}^{\mu \alpha \beta}\left(p_{1}, p_{2}\right)$ and applying the projection suggested by Eq. (4.21), we will have

$$
\lim _{p_{1}^{2} \rightarrow 0} \lim _{p_{2}^{2} \rightarrow 0} p_{1 \alpha} p_{2 \beta} \mathcal{T}^{\mu \alpha \beta}\left(p_{1}, p_{2}\right)=\frac{1}{2 g_{5}^{2}}\left(p_{1}+p_{2}\right)^{\mu} Q^{2} J(Q),
$$

where $J(Q)$ is the dynamic factor given by the convolution

$$
J(Q)=\int_{\epsilon}^{z_{0}} \frac{d z}{z} \mathcal{J}(Q, z) \psi^{2}(z)
$$

\subsubsection{Dynamic Factor and Wave Functions}

The vector bulk-to-boundary propagator $\mathcal{J}(Q, z) \equiv \mathcal{V}(i Q, z)$ for spacelike momenta, entering into the dynamic factor $J(Q)$, satisfies the equation

$$
z \partial_{z}\left(\frac{1}{z} \partial_{z} \mathcal{J}(Q, z)\right)=Q^{2} \mathcal{J}(Q, z)
$$


with b.c. $\mathcal{J}(Q, 0)=1$ and $\partial_{z} \mathcal{J}\left(Q, z_{0}\right)=0$. Its explicit form is given by

$$
\mathcal{J}(Q, z)=Q z\left[K_{1}(Q z)+I_{1}(Q z) \frac{K_{0}\left(Q z_{0}\right)}{I_{0}\left(Q z_{0}\right)}\right] .
$$

One can easily see that $\mathcal{J}(0, z)=1$. Combining all the factors, we get

$$
f_{\pi}^{2} F_{\pi}^{\left(F^{2}\right)}\left(Q^{2}\right)=\frac{1}{2 g_{5}^{2}} Q^{2} \int_{0}^{z_{0}} \frac{d z}{z} \mathcal{J}(Q, z) \psi^{2}(z)
$$

Integrating by parts and using equations of motion both for $\mathcal{J}$ and $\psi$ gives

$$
F_{\pi}^{\left(F^{2}\right)}\left(Q^{2}\right)=\frac{1}{g_{5}^{2} f_{\pi}^{2}} \int_{0}^{z_{0}} d z z \mathcal{J}(Q, z)\left[\left(\frac{\partial_{z} \psi}{z}\right)^{2}+\frac{g_{5}^{2} v^{2}}{z^{4}} \psi(\psi-\pi)\right] .
$$

We need also to add the $V \pi \pi$ contribution from the $|D X|^{2}$ term of the AdS action (5.1). It is generated by

$$
\left.S_{\mathrm{AdS}}^{|D X|^{2}}\right|_{V \pi \pi}=\epsilon_{a b c} \int d^{4} x d z\left[\frac{v^{2}(z)}{z^{3}}\left(A_{M}^{a}-\partial_{M} \pi^{a}\right) \pi^{b} V^{c M}\right],
$$

and its inclusion changes $\psi(\psi-\pi)$ into $(\psi-\pi)^{2}$ in Eq. (4.36). The total result (see also Ref. [26]) may be now conveniently expressed in terms of the $\Psi \equiv \psi-\pi$ wave function

$$
F_{\pi}\left(Q^{2}\right)=\frac{1}{g_{5}^{2} f_{\pi}^{2}} \int_{0}^{z_{0}} d z z \mathcal{J}(Q, z)\left[\left(\frac{\partial_{z} \Psi}{z}\right)^{2}+\frac{g_{5}^{2} v^{2}}{z^{4}} \Psi^{2}(z)\right]
$$

Using equation of motion for $\Psi(z)$, one can see that the expression in square brackets coincides with

$$
\frac{1}{z} \partial_{z}\left(\Psi(z) \frac{1}{z} \partial_{z} \Psi(z)\right)=-g_{5}^{2} f_{\pi}^{2} \frac{1}{z} \partial_{z}(\Psi(z) \Phi(z))
$$

and write the form factor as

$$
F_{\pi}\left(Q^{2}\right)=-\int_{0}^{z_{0}} d z \mathcal{J}(Q, z) \partial_{z}(\Psi(z) \Phi(z))
$$

This representation allows one to easily check the normalization

$$
F_{\pi}(0)=-\int_{0}^{z_{0}} d z \partial_{z}(\Psi(z) \Phi(z))=\Psi(0) \Phi(0)=1
$$

where we took into account that $\mathcal{J}(0, z)=1$ and $\Phi\left(z_{0}\right)=0$. We can also represent our result for the pion form factor as

$$
F_{\pi}\left(Q^{2}\right)=\int_{0}^{z_{0}} d z z \mathcal{J}(Q, z)\left[g_{5}^{2} f_{\pi}^{2} \Phi^{2}(z)+\frac{\sigma^{2}}{f_{\pi}^{2}} z^{2} \Psi^{2}(z)\right] \equiv \int_{0}^{z_{0}} d z z \mathcal{J}(Q, z) \rho(z)
$$


and interpret the function $\rho(z)$ as the radial distribution density, as was done in Refs. [36, 37]. Note that keeping only the first term in square brackets gives an expression similar to our result [36] for the $\rho$-meson form factor

$$
\mathcal{F}_{11}\left(Q^{2}\right)=\int_{0}^{z_{0}} d z z \mathcal{J}(Q, z)\left|\phi_{1}(z)\right|^{2}
$$

in terms of the function $\phi_{1}$ conjugate to the solution of the basic equation of motion. The value of $\phi_{1}(z)$ at the origin is proportional to the $\rho$-meson decay constant $f_{\rho} / m_{\rho} \equiv g_{\rho}$ (experimentally, $g_{\rho}^{\exp } \approx 207 \mathrm{MeV}[48]$ ), namely, $\phi_{1}(0)=g_{5} g_{\rho}$. Thus, the pion wave function $g_{5} f_{\pi} \Phi(z) \equiv \phi_{\pi}(z)$ is a direct analog of the $\rho$-meson wave function $\phi_{1}(z)$. The main difference is that, in the pion case, there is also the second term in the form factor expression. The latter, in fact, is necessary to secure correct normalization of the form factor at $Q^{2}=0$. In Eq. (4.38), this term is written in terms of the $\Psi(z)$ wave function, but using Eq. (4.17) we can rewrite it also in terms of $\Phi(z)$ or $\phi_{\pi}(z)$ :

$$
\rho(z)=\phi_{\pi}^{2}(z)+\frac{1}{g_{5}^{2} \sigma^{2}}\left(\frac{1}{z^{2}} \partial_{z} \phi_{\pi}(z)\right)^{2} .
$$

\subsection{Wave Functions and Form Factor}

\subsubsection{Structure of Pion Wave Functions}

Explicit form of the $\Psi$ wave function follows from the solution of Eq. (4.12):

$$
\Psi(z)=z \Gamma[2 / 3]\left(\frac{\alpha}{2}\right)^{1 / 3}\left[I_{-1 / 3}\left(\alpha z^{3}\right)-I_{1 / 3}\left(\alpha z^{3}\right) \frac{I_{2 / 3}\left(\alpha z_{0}^{3}\right)}{I_{-2 / 3}\left(\alpha z_{0}^{3}\right)}\right]
$$

where $\alpha=g_{5} \sigma / 3 \approx 1.481 \sigma$ (recall that $g_{5}=\sqrt{2} \pi$, see e.g. Ref.[37]). As a result, $\Phi(z)$ is given by

$$
\begin{aligned}
\Phi(z) & =-\frac{1}{g_{5}^{2} f_{\pi}^{2}}\left(\frac{1}{z} \partial_{z} \Psi(z)\right) \\
& =\frac{3 z^{2}}{g_{5}^{2} f_{\pi}^{2}} \Gamma[2 / 3]\left(\frac{\alpha^{4}}{2}\right)^{1 / 3}\left[-I_{2 / 3}\left(\alpha z^{3}\right)+I_{-2 / 3}\left(\alpha z^{3}\right) \frac{I_{2 / 3}\left(\alpha z_{0}^{3}\right)}{I_{-2 / 3}\left(\alpha z_{0}^{3}\right)}\right] .
\end{aligned}
$$

This formula, combined with Eq. (4.16), establishes the relation

$$
f_{\pi}^{2}=3 \cdot 2^{1 / 3} \frac{\Gamma[2 / 3]}{\Gamma[1 / 3]} \frac{I_{2 / 3}\left(\alpha z_{0}^{3}\right)}{I_{-2 / 3}\left(\alpha z_{0}^{3}\right)} \frac{\alpha^{2 / 3}}{g_{5}^{2}}
$$

for $f_{\pi}$ in terms of the condensate parameter $\alpha$ and the confinement radius $z_{0}$. Since $\sigma$ appears in the solutions only through $\alpha$, we will use $\alpha$ in what follows. Note also that $\alpha^{1 / 3} \approx 1.14 \sigma^{1 / 3}$. 
Realizing that the equations of motion for the vector sector in this holographic model are not affected by the chiral symmetry-breaking effects expressed through the function $v(z)$, it is natural to set the value of $z_{0}$ from the vector sector spectrum, i.e., by the $\rho$-meson mass. The numerical value of $z_{0}$ (call it $z_{0}^{\rho}$ ) is then $z_{0}^{\rho} \approx 1 / 323 \mathrm{MeV}$. As given by Eq. (4.46), $f_{\pi}$ looks like a rather complicated function of two scales, $z_{0}$ and $\alpha$. Note, however, that the ratio $I_{2 / 3}(a) / I_{-2 / 3}(a)$ is very close to 1 for $a \gtrsim 2$ and practically indistinguishable from 1 for $a \gtrsim 3$. Hence, for sufficiently large values of the confinement radius, $z_{0} \gtrsim 1 / \alpha^{1 / 3}$, the value of $f_{\pi}$ is determined by the value of $\alpha$ alone. This limiting value of $f_{\pi}$ is given by

$$
\left.f_{\pi}\right|_{z_{0} \rightarrow \infty}=2^{1 / 6} \frac{\alpha^{1 / 3}}{g_{5}} \sqrt{\frac{3 \Gamma[2 / 3]}{\Gamma[1 / 3]}}=\frac{3^{1 / 2}}{2^{1 / 3} \pi} \sqrt{\frac{\Gamma[2 / 3]}{\Gamma[1 / 3]}} \alpha^{1 / 3} \approx \frac{\alpha^{1 / 3}}{3.21} .
$$

Requiring that $\left.f_{\pi}\right|_{z_{0} \rightarrow \infty}$ coincides with the experimental value, $f_{\pi} \approx 131 \mathrm{MeV}$, one should take $\alpha^{1 / 3} \approx 420 \mathrm{MeV}$. For such $\alpha$, the value of $1 / \alpha^{1 / 3}$ is close to $z_{0}^{\rho}$, i.e., we are in the region $\alpha z_{0}^{3} \sim 1$ and we may expect that, even if we use exact formula (4.46) with $z_{0}=z_{0}^{\rho}$, the value of $f_{\pi}$ would not change much. Indeed, to get $f_{\pi} \approx$ $131 \mathrm{MeV}$ from Eq. (4.46) for $1 / z_{0}=323 \mathrm{MeV}$, we should take $\alpha^{1 / 3} \approx 424 \mathrm{MeV} \equiv$ $\alpha_{0}^{1 / 3}$. Thus, in this range of parameters, the value of $f_{\pi}$ is practically in one-to-one correspondence with the value of $\alpha$. It is convenient to introduce a dimensionless variable

$$
a \equiv \alpha z_{0}^{3}=\frac{1}{3} g_{5} \sigma z_{0}^{3}
$$

Then the values $\alpha_{0}^{1 / 3}=424 \mathrm{MeV}$ and $1 / z_{0}^{\rho}=323 \mathrm{MeV}$ correspond to $a=2.26 \equiv a_{0}$. As one can see from Fig.(4.1), the dependence of $f_{\pi}$ is practically flat for $a \gtrsim 2$.

The confinement radius $z_{0}$ presents a natural scale to measure length, so it makes sense to rewrite the form factor formula (4.38) as an integral over the dimensionless variable $\zeta \equiv z / z_{0}$ :

$$
\begin{aligned}
F_{\pi}\left(Q^{2}\right) & =3 \int_{0}^{1} d \zeta \zeta \mathcal{J}\left(Q, \zeta, z_{0}\right)\left[n(a) \varphi^{2}(\zeta, a)+\frac{a^{2} \zeta^{2}}{n(a)} \psi^{2}(\zeta, a)\right] \\
& \equiv \int_{0}^{1} d \zeta \zeta \mathcal{J}\left(Q, \zeta, z_{0}\right) \rho(\zeta, a),
\end{aligned}
$$

where the mass scale $\alpha$ is reflected by the dimensionless parameter $a$. The factor $n(a)$ takes care of the correct normalization of the form factor. It is given by

$$
n(a)=2^{1 / 3} a^{2 / 3} \frac{\Gamma[2 / 3]}{\Gamma[1 / 3]} \frac{I_{2 / 3}(a)}{I_{-2 / 3}(a)} .
$$

For small $a$, it may be approximated by $\frac{3}{4} a^{2}$. For large $a$, using the fact that $I_{2 / 3}(a) / I_{-2 / 3}(a)$ is very close to 1 for $a \gtrsim 2$, we may approximate $n(a) \approx 0.637 a^{2 / 3}$ in this region. In terms of $n(a)$, the pion decay constant can be written as

$$
f_{\pi}=\frac{1}{\pi a^{1 / 3}} \sqrt{\frac{3}{2} n(a)} \alpha^{1 / 3} .
$$




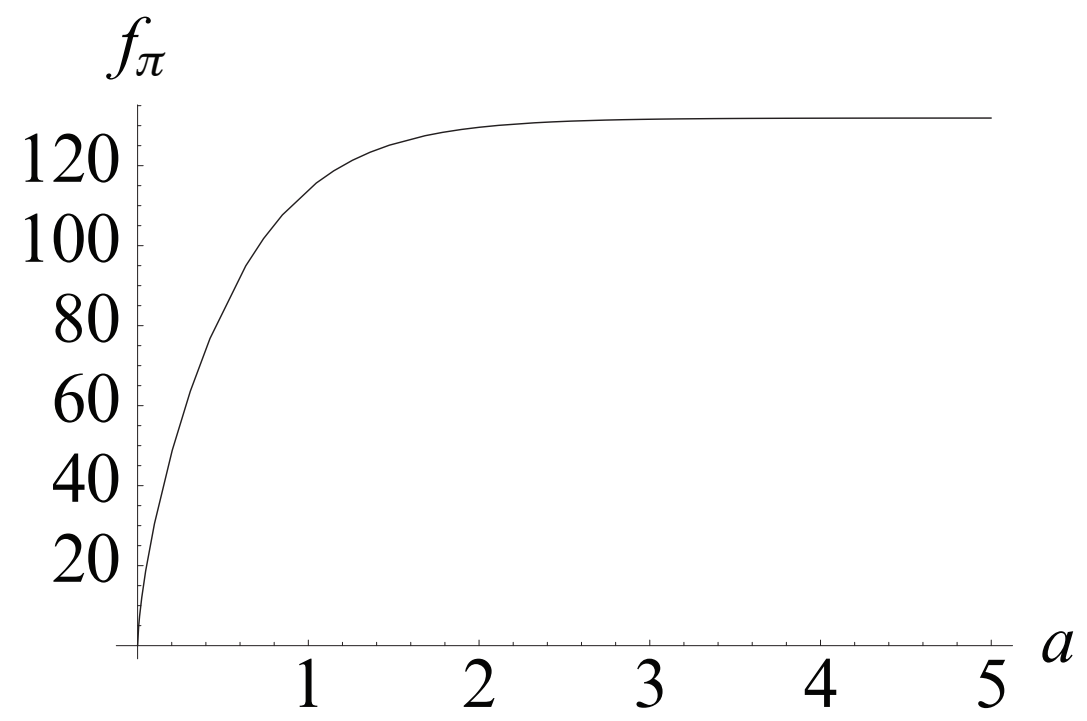

Figure 4.1: Pion decay constant $f_{\pi}$ as a function of $a$ for fixed $\alpha^{1 / 3}=424 \mathrm{MeV}$.

For large $a$, this gives

$$
\left.f_{\pi}\right|_{a \gtrsim 2} \approx 0.311 \alpha^{1 / 3} .
$$

For small $a$, we have

$$
\left.f_{\pi}\right|_{a \lesssim 1}=\frac{3 a^{2 / 3}}{2 \sqrt{2} \pi} \alpha^{1 / 3}+\ldots \approx 0.338 \alpha z_{0}^{2}=0.338 \frac{a}{z_{0}} .
$$

The functions $\varphi(\zeta, a), \psi(\zeta, a)$ are just the $\Phi$ and $\Psi$ wave functions written in $\zeta$ and $a$ variables. For $a=0$, the limiting forms are $\varphi(\zeta, 0)=1-\zeta^{4}$ and $\psi(\zeta, 0)=1$. As $a$ increases, both functions become more and more narrow (see Fig.4.3).

For density, we have $\rho(\zeta, a=0)=4 \zeta^{2}$ in the $a \rightarrow 0$ limit, a function that vanishes at the origin (see Fig.(4.4)). For nonzero $a$, the value of $\rho(\zeta=0, a)$ monotonically increases with $a$, and the function itself narrows.

The increase of $\rho(\zeta=0, a)$ with $a$ is generated by the monotonically increasing function $n(a)$. It is interesting to compare the pion density $\rho(\zeta, 2.26)$ (taken at the "experimental" value $a=2.26)$ with the $\rho$-meson density $\rho_{\rho}(\zeta)$ of Ref. [36]. These densities are rather close for $\zeta>0.5$, but strongly differ for small $\zeta$. In particular, the $\rho$-meson density is more than two times larger for $\zeta=0$, which corresponds to the hard-wall model result that $g_{\rho}$ is essentially larger than $f_{\pi}$.

\subsubsection{Pion Charge Radius}

It is interesting to investigate how well these values $z_{0}=1 / 323 \mathrm{MeV}$ and $\alpha=$ $(424 \mathrm{MeV})^{3}$ describe another important low-energy characteristic of the pion - its 


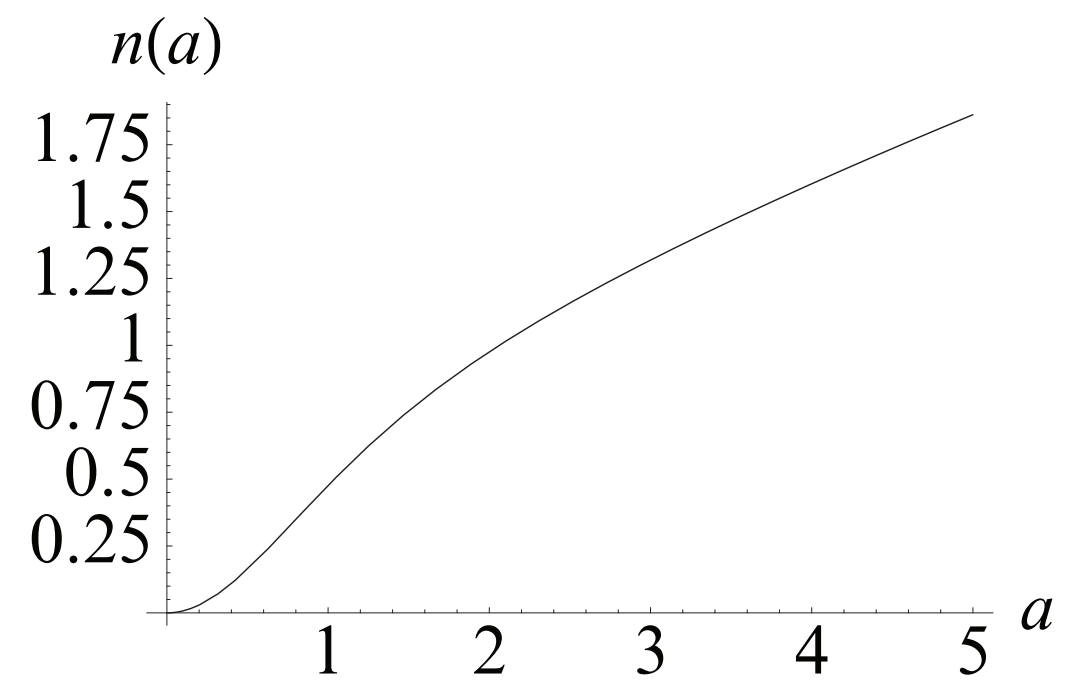

Figure 4.2: Function $n(a)$.

charge radius. Using the $Q^{2}$-expansion of the vector source [36]

$$
\mathcal{J}\left(Q, \zeta, z_{0}\right)=1-\frac{Q^{2}}{4} z_{0}^{2} \zeta^{2}[1-2 \ln \zeta]+\ldots
$$

and an explicit form of the density

$$
\begin{aligned}
\rho(\zeta, a)=\frac{3}{2} \Gamma(1 / 3) \Gamma(2 / 3) a^{2} \zeta^{4} & {\left[\left(\nu(a) I_{-2 / 3}\left(a \zeta^{3}\right)-\frac{I_{2 / 3}\left(a \zeta^{3}\right)}{\nu(a)}\right)^{2}\right.} \\
+ & \left.\left(\frac{I_{-1 / 3}\left(a \zeta^{3}\right)}{\nu(a)}-\nu(a) I_{1 / 3}\left(a \zeta^{3}\right)\right)^{2}\right],
\end{aligned}
$$

where $\nu(a) \equiv \sqrt{I_{2 / 3}(a) / I_{-2 / 3}(a)}$, we obtain for the pion charge radius:

$$
\left\langle r_{\pi}^{2}\right\rangle=\frac{3}{2} z_{0}^{2} \int_{0}^{1} d \zeta \zeta^{3}[1-2 \ln \zeta] \rho(\zeta, a)=\frac{4}{3} z_{0}^{2}\left\{1-\frac{a^{2}}{4}+\mathcal{O}\left(a^{4}\right)\right\} .
$$

Hence, for fixed $z_{0}$ and small $a$, when $\alpha \ll 1 / z_{0}^{3}$, the pion radius is basically determined by the confinement scale $z_{0}$. In particular, $\left\langle r_{\pi}^{2}\right\rangle=\frac{4}{3} z_{0}^{2}$ for $\alpha=0$. Numerically, taking $z_{0}=z_{0}^{\rho} \approx 1 / 323 \mathrm{MeV}=0.619 \mathrm{fm}$, we obtain $\left\langle r_{\pi}^{2}\right\rangle=0.51 \mathrm{fm}^{2}$. This result is very close to the value $\left\langle r_{\rho}^{2}\right\rangle_{C} \approx 0.53 \mathrm{fm}^{2}$ that we obtained in the hardwall model for the $\rho$-meson electric radius determined in [36] from the slope of the $G_{C}\left(Q^{2}\right)$ form factor. However, since $G_{C}\left(Q^{2}\right)$ involves kinematic-type terms $Q^{2} / m_{\rho}^{2}$, it seems more appropriate to compare $F_{\pi}\left(Q^{2}\right)$ with the $\mathcal{F}_{11}\left(Q^{2}\right)$ form factor (4.42) given directly by a wave function overlap integral. The slope of $\mathcal{F}_{11}\left(Q^{2}\right)$ is smaller 

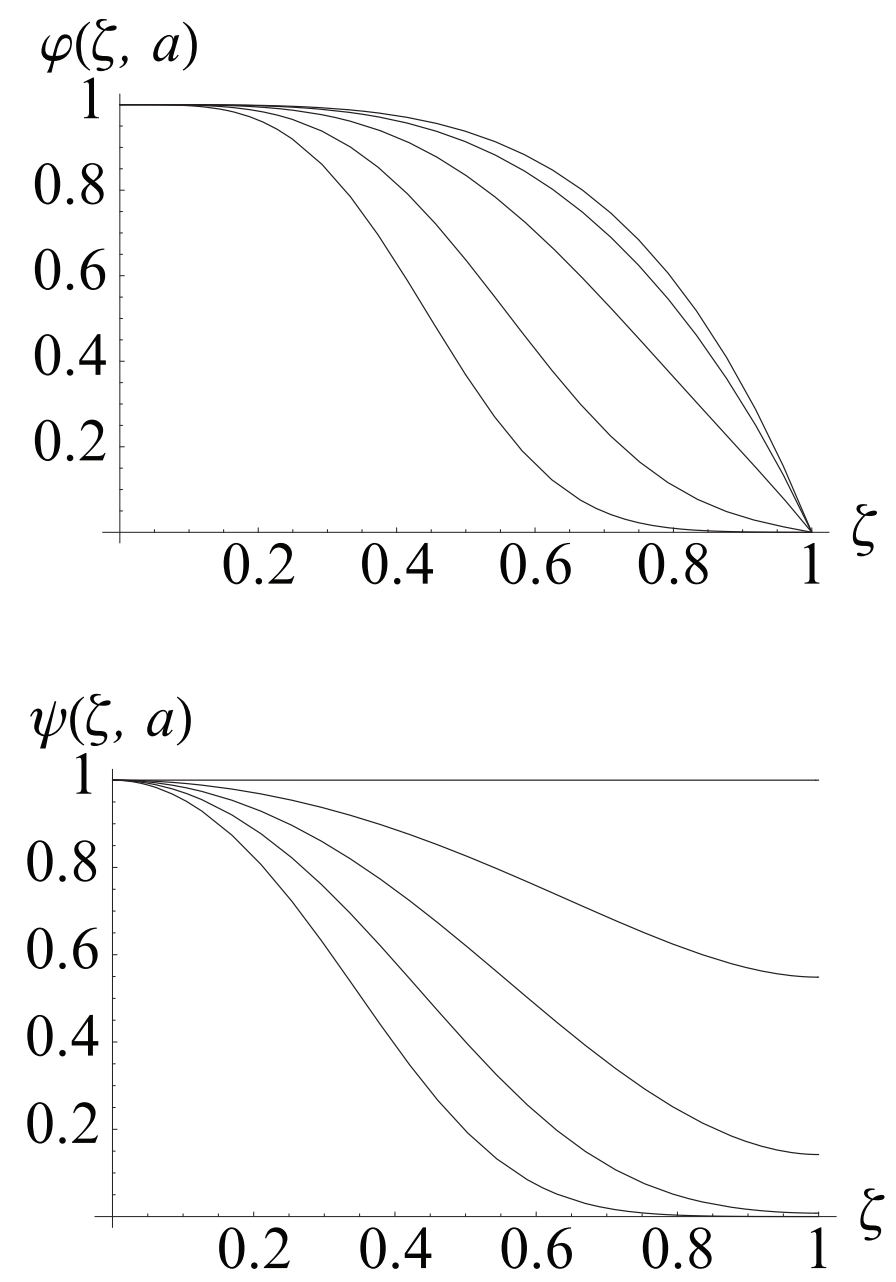

Figure 4.3: Functions $\varphi(\zeta, a)$ (top) and $\psi(\zeta, a)$ (bottom) for several values of $a$ : $a=0$ (uppermost lines), $a=1, a=2.26, a=5, a=10$ (lowermost lines).

than that of $G_{C}\left(Q^{2}\right)$, and the corresponding radius is also smaller: $\left\langle r_{\rho}^{2}\right\rangle_{\mathcal{F}}=0.27 \mathrm{fm}^{2}$. Thus, for $\alpha=0$, the pion r.m.s. radius is about 1.4 times larger than the $\rho$-meson size determined by $\left\langle r_{\rho}^{2}\right\rangle_{\mathcal{F}}^{1 / 2}$.

With the increase of $\alpha$, the pion becomes smaller (see Fig.4.5). The experimental value of $0.45 \mathrm{fm}^{2}$ [48] is reached for $a \sim 0.9$. However, the corresponding value $f_{\pi} \approx 80 \mathrm{MeV}$ is too small. If we take $a=a_{0}=2.26$, then $\left\langle r_{\pi}^{2}\right\rangle=0.34 \mathrm{fm}^{2}$. Thus, if we insist on using $z_{0}=z_{0}^{\rho}$ dictated by the hard-wall model calculation of the $\rho$-meson mass, and the value of $\alpha$ producing the experimental $f_{\pi}$ (note that then $\alpha^{-2 / 3} \approx 0.222 \mathrm{fm}^{2}$ ), the pion radius is smaller than the experimental value. In linear units, the difference, in fact, does not look very drastic: just $0.58 \mathrm{fm}$ instead of 0.66 fm. Given that the hard-wall model for confinement is rather crude, the agreement may be considered as encouraging. Furthermore, one may expect that, in a more 


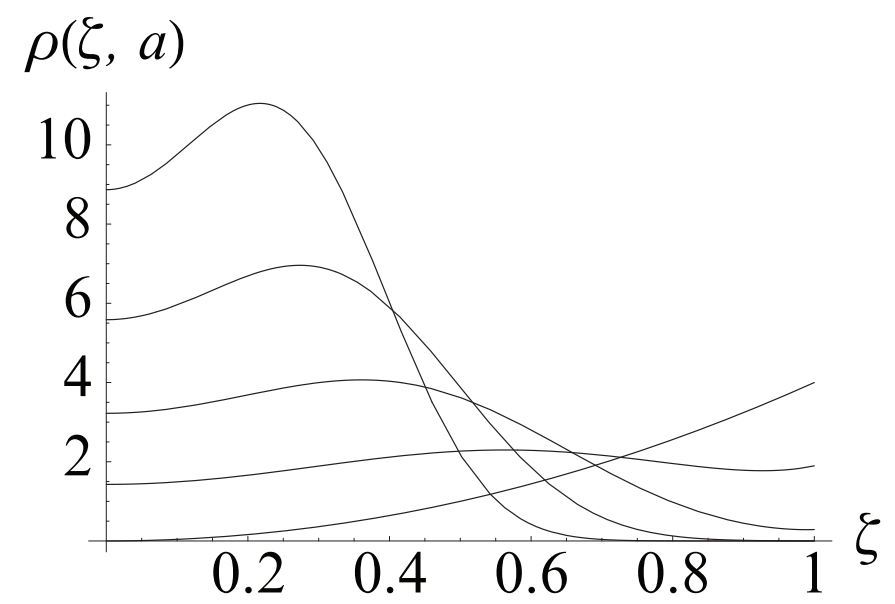

$\rho(\zeta, a)$

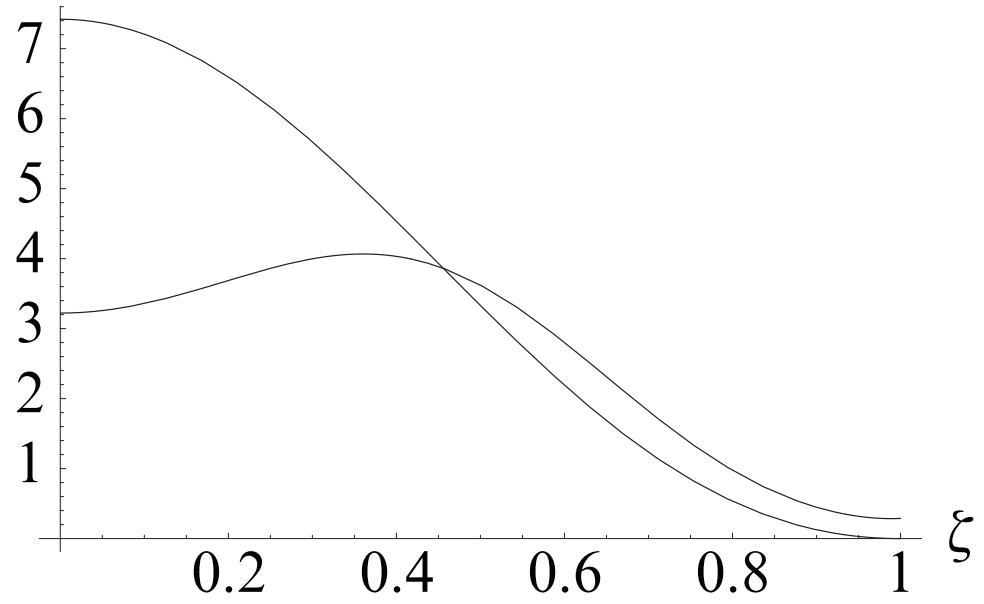

$\zeta \rho(\zeta, a)$

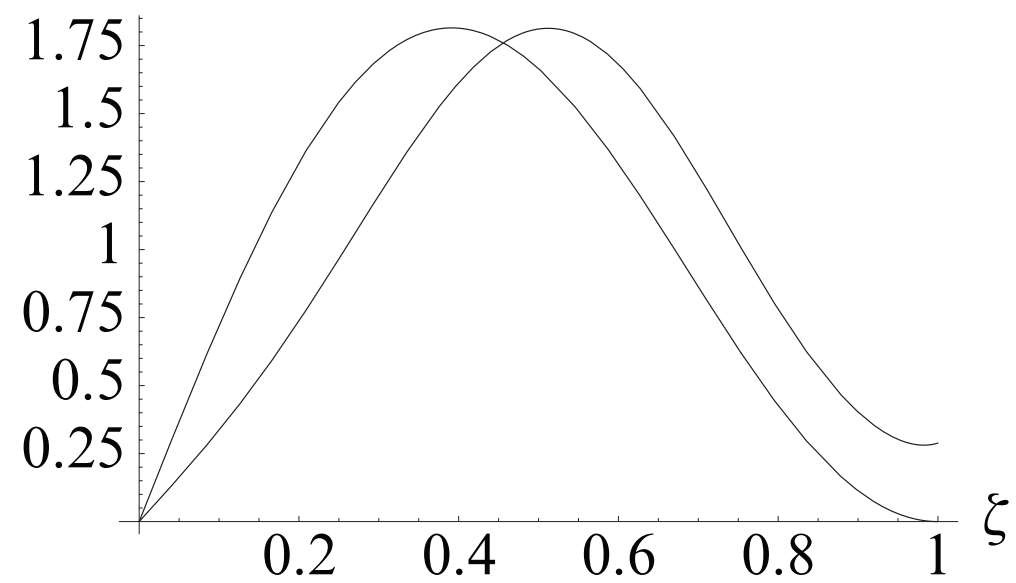

Figure 4.4: Top: Function $\rho(\zeta, a)$ for $a=0, a=1, a=2.26, a=5, a=10$. Middle: Densities $\rho(\zeta, 2.26)$ for pion and $\rho_{\rho}(\zeta)$ for $\rho$-meson in the hard-wall model. Bottom: Same for densities multiplied by $\zeta$. 


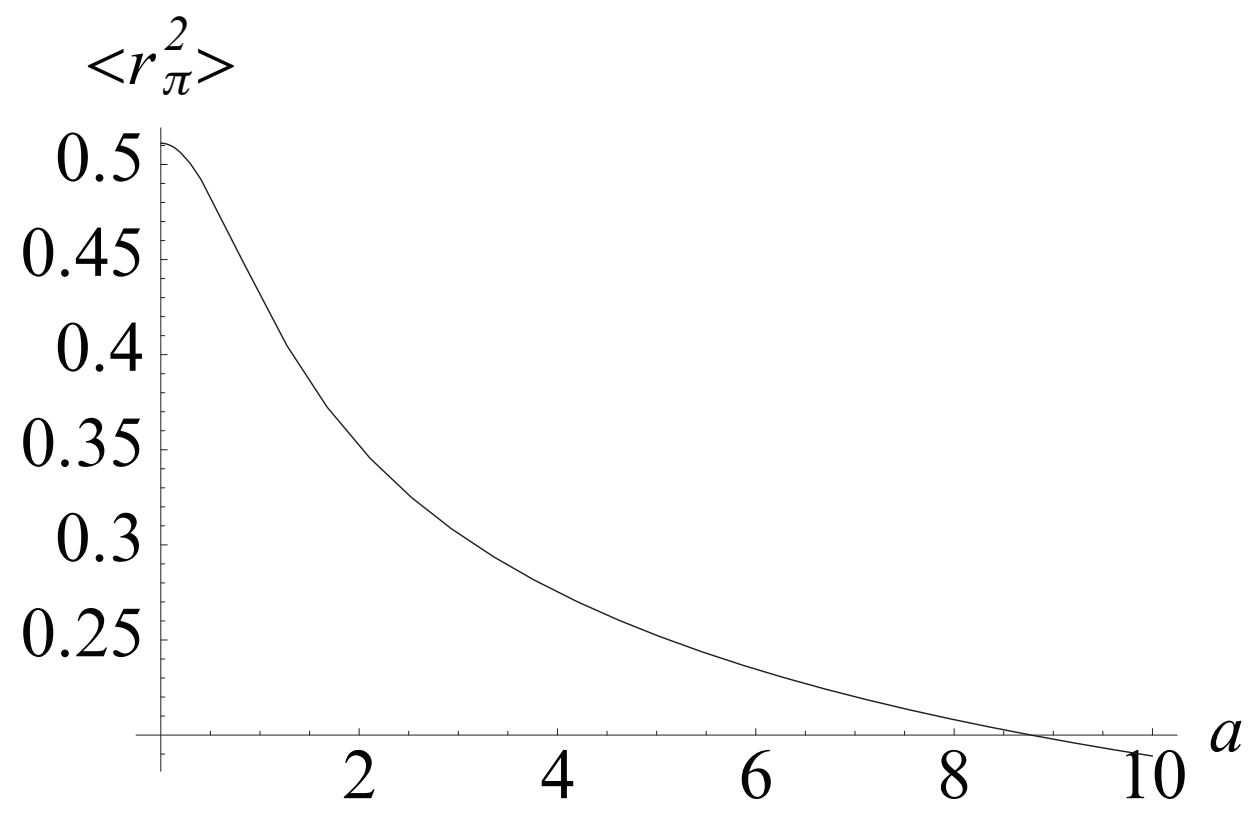

Figure 4.5: $\left\langle r_{\pi}^{2}\right\rangle$ in $\mathrm{fm}^{2}$ for $z_{0}=z_{0}^{\rho}$ as a function of $a$.

realistic softer model of confinement, the size of the pion will be larger. Such an expectation is supported by our soft-wall model calculation of the $\rho$-meson electric radius, for which we obtained $\left\langle r_{\rho}^{2}\right\rangle_{C}=0.66 \mathrm{fm}^{2}\left(0.40 \mathrm{fm}^{2}\right.$ for $\left.\left\langle r_{\rho}^{2}\right\rangle_{\mathcal{F}}\right)$, i.e., the result by $0.13 \mathrm{fm}^{2}$ larger than in the hard-wall model. If $\left\langle r_{\pi}^{2}\right\rangle$ would increase by a similar amount, the result would be very close to the quoted experimental value.

To find $\left\langle r_{\pi}^{2}\right\rangle$ for large $a$ (i.e., when $\alpha \gtrsim z_{0}^{-3}$ for fixed $z_{0}$, or when $z_{0} \gtrsim \alpha^{-1 / 3}$ for fixed $\alpha$ ), we use first the observation that, in the region $a \gtrsim 2$, we may approximate $\nu(a) \approx 1$. Then the factor in square brackets in Eq. (4.55) becomes a function of the combination $a \zeta^{3} \equiv \mu$ (call it $R(\mu)$ ), and we can write

$$
\left.\left\langle r_{\pi}^{2}\right\rangle\right|_{a \gtrsim 2} \approx \frac{3}{4} \Gamma(1 / 3) \Gamma(2 / 3)\left(\frac{1}{\alpha}\right)^{2 / 3} \int_{0}^{a} d \mu \mu^{5 / 3} R(\mu)\left[1-\frac{2}{3} \ln \frac{\mu}{a}\right] .
$$

For $a \gtrsim 2$, the upper limit of integration in this expression may be safely substituted by infinity producing

$$
\begin{aligned}
& \int_{0}^{\infty} d \mu \mu^{5 / 3} R(\mu)=\frac{2^{2 / 3}}{3 \Gamma^{2}(2 / 3)} \equiv G \\
& \int_{0}^{\infty} d \mu \mu^{5 / 3} R(\mu) \ln \mu \approx G \ln 0.566
\end{aligned}
$$

which gives

$$
\left.\left\langle r_{\pi}^{2}\right\rangle\right|_{a \gtrsim 2}=\frac{\Gamma(1 / 3)}{2^{4 / 3} \Gamma(2 / 3)}\left(\frac{1}{\alpha}\right)^{2 / 3}\left[1+\frac{2}{3} \ln \left(\frac{a}{0.566}\right)\right]
$$


Using Eq. (4.47), we can express the coefficient in front of the square bracket in terms of $f_{\pi}$ :

$$
\left.\left\langle r_{\pi}^{2}\right\rangle\right|_{a \gtrsim 2}=\frac{3}{4 \pi^{2} f_{\pi}^{2}}+\frac{1}{2 \pi^{2} f_{\pi}^{2}} \ln \left(\frac{\alpha z_{0}^{3}}{0.566}\right) .
$$

Thus, $\left\langle r_{\pi}^{2}\right\rangle$ in the $a \gtrsim 2$ region consists of two componens: a fixed term $3 / 4 \pi^{2} f_{\pi}^{2}$ and a term logarithmically increasing with $z_{0}$. As $z_{0} \rightarrow \infty$, the pion charge radius becomes infinite, reflecting the fact that the pion in this model is massless. A similar structure in the expression for the pion charge radius was obtained [51] in the Nambu-Jona-Lasinio (NJL) model

$$
\left\langle r_{\pi}^{2}\right\rangle_{N J L}=\frac{3}{2 \pi^{2} f_{\pi}^{2}}+\frac{1}{8 \pi^{2} f_{\pi}^{2}} \ln \left(\frac{m_{\sigma}^{2}}{m_{\pi}^{2}}\right) .
$$

It also has the logarithmic term $\ln m_{\pi}^{2}[52,53]$ resulting in the infinite radius for massless pion and the infrared-finite piece $3 / 2 \pi^{2} f_{\pi}^{2}[54,55]$. The latter, however, is twice larger than that in our result (4.60) and contributes $0.34 \mathrm{fm}^{2}$ to $\left\langle r_{\pi}^{2}\right\rangle$, with the chiral logarithm term producing the extra $0.11 \mathrm{fm}^{2}$ required for agreement with experiment. In our case, the logarithmic term taken for $a=a_{0}$ is approximately equal to $3 / 4 \pi^{2} f_{\pi}^{2}$, thus almost doubling the outcome value for $\left\langle r_{\pi}^{2}\right\rangle$. More precisely, we can write

$$
\left.\left\langle r_{\pi}^{2}\right\rangle\right|_{a \gtrsim 2}=\frac{3}{2 \pi^{2} f_{\pi}^{2}}\left[1+\frac{1}{3} \ln \left(\frac{a}{2.54}\right)\right] .
$$

For $a=2.26$, the modified logarithmic term gives a very small contribution, and our net result is very close to the value given by the NJL fixed term. Numerically, though, this prediction of the hard-wall AdS/QCD model, as we have seen, is essentially smaller than the experimental value.

\subsubsection{Form Factor at Large $Q^{2}$}

In the large- $Q^{2}$ limit, the source $\mathcal{J}(Q, z)$ is given by its free-field version $z Q K_{1}(Q z)$ that behaves asymptotically like $e^{-Q z}$. As a result, only small values $z \sim 1 / Q$ are important in the form factor integral, and the large- $Q^{2}$ asymptotic behavior of the form factor is determined by the value of $\rho(z)$ at the origin $[23,24,37]$, namely,

$$
F_{\pi}\left(Q^{2}\right) \rightarrow \frac{2 \rho(0)}{Q^{2}}=\frac{2 \phi_{\pi}^{2}(0)}{Q^{2}}=\frac{4 \pi^{2} f_{\pi}^{2}}{Q^{2}} \equiv \frac{s_{0}}{Q^{2}}
$$

Note that the combination $4 \pi^{2} f_{\pi}^{2} \equiv s_{0} \approx 0.68 \mathrm{GeV}^{2}$ frequently appears in the pion studies. In particular, it is the basic scale of the pion wave function in the local quark-hadron duality model $[124,56]$, where it corresponds to the "pion duality interval".

The leading contribution comes entirely from the $\Phi^{2}$ term of the form factor integral (4.41) while the $\Psi^{2}$ term contribution behaves asymptotically like $1 / Q^{4}$ 

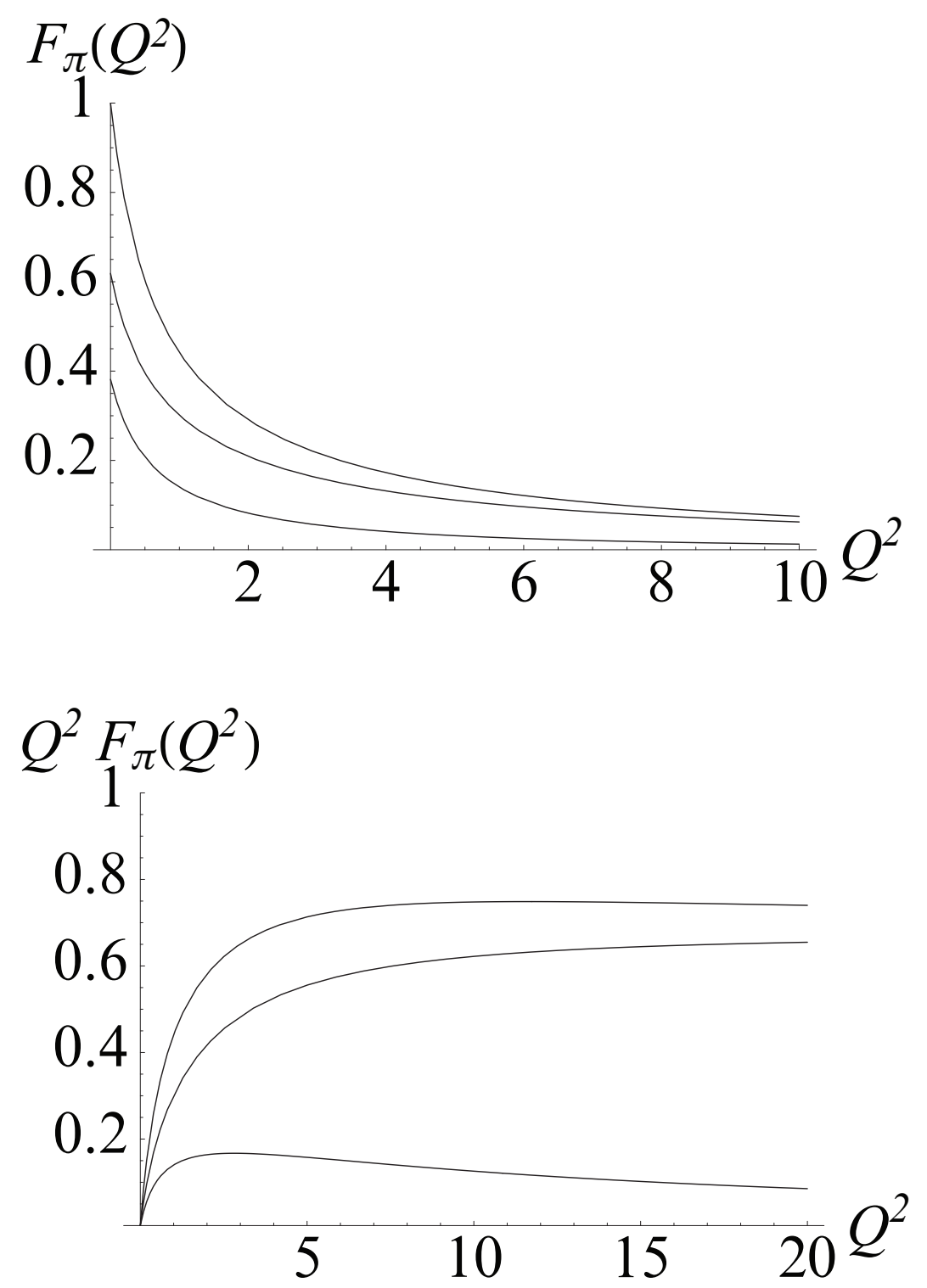

Figure 4.6: Top: Contributions to pion form factor $F_{\pi}\left(Q^{2}\right)$ from $\Psi^{2}$-term (lower curve), from $\Phi^{2}$-term (middle curve) and total contribution (upper curve). Bottom: Same for $Q^{2} F_{\pi}\left(Q^{2}\right)$. 
since it is accompanied by extra $z^{2}$ factor. Note, however, that it is quite visible in the experimentally interesting region $Q^{2} \lesssim 10 \mathrm{GeV}^{2}$ : it is responsible for more than $20 \%$ of the form factor value in this region (moreover, at $Q^{2}=0$, the $\Psi^{2}$ term contributes about $40 \%$ into the normalization of the form factor).

From a phenomenological point of view, different AdS/QCD-like models for the pion form factor differ in the shape of the density $\rho(\zeta)$ that they produce. If we require that the density $\rho(z)$ equals $2 \pi^{2} f_{\pi}^{2}$ at the origin, the asymptotic behavior is $F_{\pi}\left(Q^{2}\right) \rightarrow s_{0} / Q^{2}$ in any such model. For $Q^{2}=0$, the form factor is normalized to one, so basically the models would differ in how they interpolate between these two limits. In particular, the simplest interpolation is provided by the monopole formula

$$
F_{\pi}^{\text {mono }}\left(Q^{2}\right)=\frac{1}{1+Q^{2} / s_{0}}
$$

while our hard-wall calculation gives a curve that goes above $F_{\pi}^{\text {mono }}\left(Q^{2}\right)$ : the ratio $F_{\pi}\left(Q^{2}\right) / F_{\pi}^{\text {mono }}\left(Q^{2}\right)$ is larger than 1 for all $Q^{2}>0$, slowly approaching unity as $Q^{2} \rightarrow \infty$ (see Fig.4.7).

In fact, a purely monopole form factor was obtained in our paper [37], where we studied the $\rho$-meson form factors in the soft-wall holographic model, in which confinement is generated by $\sim z^{2}$ oscillator-type potential. It was shown in [37] that the form factor integral

$$
\mathcal{F}\left(Q^{2}, \kappa\right)=\int_{0}^{\infty} d z z \mathcal{J}^{\mathrm{O}}(Q, z)|\Phi(z, \kappa)|^{2}
$$

in which $\Phi(z, \kappa)=\sqrt{2} \kappa e^{-z^{2} \kappa^{2} / 2}$ is the lowest bound state wave function, and

$$
\mathcal{J}^{\mathrm{O}}(Q, z)=z^{2} \kappa^{2} \int_{0}^{1} \frac{d x}{(1-x)^{2}} x^{Q^{2} / 4 \kappa^{2}} \exp \left[-\frac{x}{1-x} z^{2} \kappa^{2}\right]
$$

is the bulk-to-boundary propagator of this oscillator-type model, is exactly equal to $1 /\left(1+Q^{2} /\left(4 \kappa^{2}\right)\right)$. The magnitude of the oscillator scale $\kappa$ was fixed in our paper [37] by the value of the $\rho$-meson mass: $\kappa=\kappa_{\rho} \equiv m_{\rho} / 2$. As a result, the form factor $\mathcal{F}\left(Q^{2}, \kappa=m_{\rho} / 2\right)$ had the $\rho$-dominance behavior $1 /\left(1+Q^{2} / m_{\rho}^{2}\right)$.

If we take $\kappa=\kappa_{\pi} \equiv \pi f_{\pi} \approx 410 \mathrm{MeV}$ both for $\Phi(z, \kappa)$ and $\mathcal{J}^{\mathrm{O}}(Q, z)$, the integral (4.65) gives $1 /\left(1+Q^{2} / s_{0}\right)$. The relevant wave function $\Phi\left(z, \kappa_{\pi}\right)$ has the expected correct normalization $\Phi\left(0, \kappa_{\pi}\right)=\sqrt{2} \pi f_{\pi}$, however, the slope $1 / s_{0}$ of $1 /\left(1+Q^{2} / s_{0}\right)$ at $Q^{2}=0$ (corresponding to $0.35 \mathrm{fm}^{2}$ for the radius squared) is smaller than that of the experimental pion form factor. Furthermore, $Q^{2} F_{\pi}^{\text {mono }}\left(Q^{2}\right)$ tends to $s_{0} \approx$ $0.68 \mathrm{GeV}^{2}$ for large $Q^{2}$, achieving values about $0.5 \mathrm{GeV}^{2}$ for $Q^{2} \sim 2 \mathrm{GeV}^{2}$, and thus exceeding by more than $25 \%$ the experimental JLab values [50] measured for $Q^{2}=1.6$ and $2.45 \mathrm{GeV}^{2}$. The authors of Ref. [49] proposed to use Eqs. (4.65),(4.66) as an AdS/QCD model for the pion form factor, with $\kappa=375 \mathrm{MeV}$ chosen so as to fit these high- $Q^{2}$ data. However, such a choice underestimates the value of $f_{\pi}^{2}$ by almost 30\%. Our opinion is that the AdS/QCD models should describe first the low-energy properties of hadrons, and the basic low-energy characteristics, such 

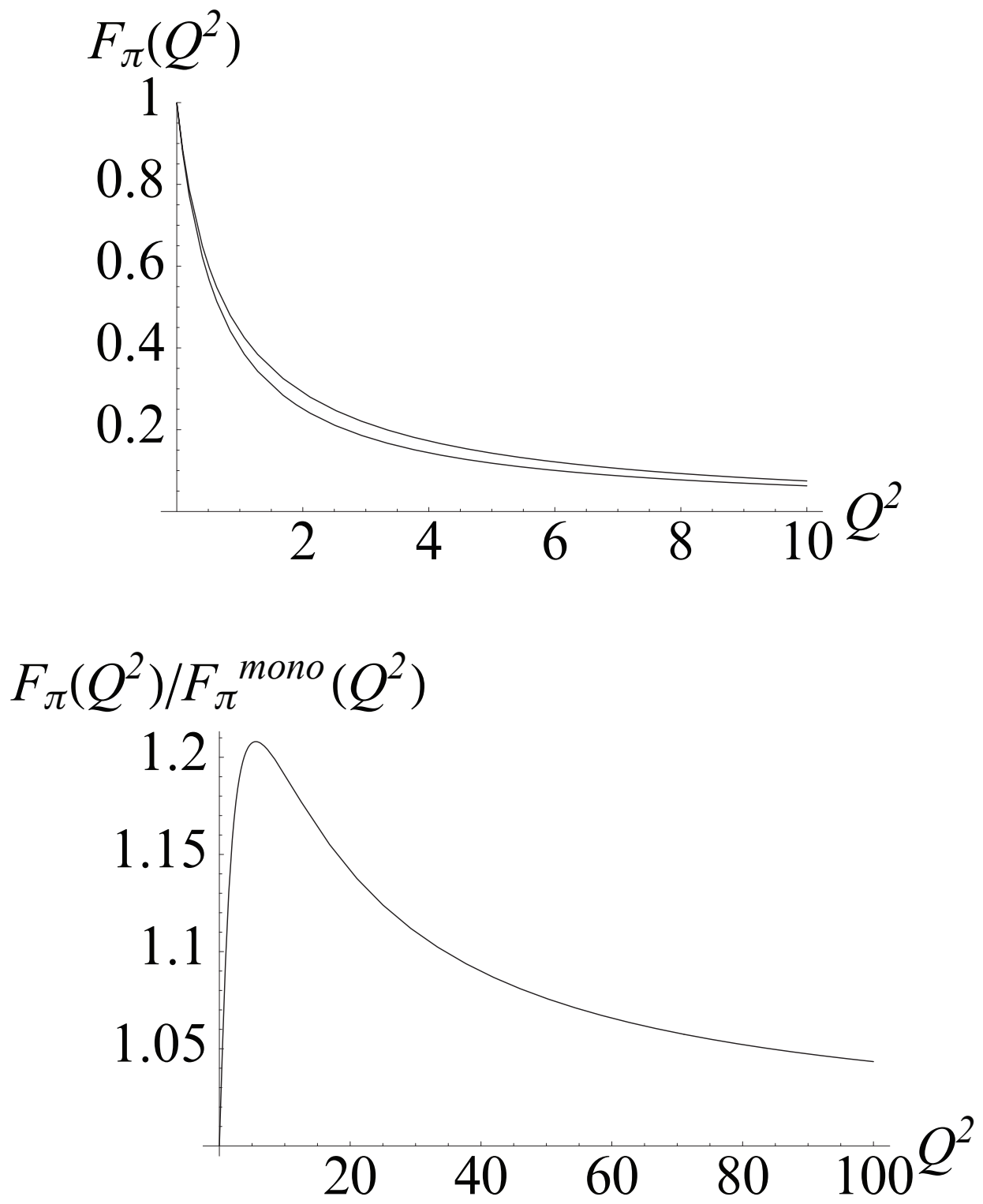

Figure 4.7: Top: Pion form factor $F_{\pi}\left(Q^{2}\right)$ from the holographic model (upper curve) in comparison with the monopole interpolation $F_{\pi}^{\text {mono }}\left(Q^{2}\right)$ (lower curve). Bottom: Ratio $F_{\pi}\left(Q^{2}\right) / F_{\pi}^{\text {mono }}\left(Q^{2}\right)$. 
as $m_{\rho}$ and $f_{\pi}$, should be used to fix the model parameters. On the other hand, if the form factor calculations based on these parameters disagree with the large$Q^{2}$ data, it is quite possible that this is just an indication that one is using the model beyond its applicability limits. Furthermore, as we have seen in the hardwall model, to correctly describe the pion one needs to include the chiral symmetry breaking effects absent in the vector channel. As a result, equations for pion wave functions are rather different from those in the $\rho$-meson case. Similarly, there are no reasons to expect that, in a soft-wall model, the pion density should have the same shape as the $\rho$-meson one. Unfortunately, the procedure of bringing in the chiral symmetry breaking effects that was used in the hard-wall model of Ref. [26] faces serious difficulties when applied to the AdS/QCD model [32] with the $z^{2}$ soft wall. As discussed in Ref. [32], the solution of the equation for the $X$ field in this model requires that chiral condensate $\sigma$ and the mass parameter $m_{q}$ are proportional to each other, so that $\sigma$ cannot be varied independently of $m_{q}$. Moreover, if one takes the chiral limit $m_{q}=0$, the chiral condensate should also vanish. This difficulty may be avoided by switching to more sophisticated recent models (cf. $[105,106,107])$ in which the chiral condensate is generated dynamically. However, such a consideration goes well beyond the scope of the present work ([38]). Thus, we just resort to an idea that whatever mechanism is involved, the net practical outcome is a particular shape of the density $\rho(z)$ that eventually determines the pion form factor and other pion characteristics. Below, we give an example of a density $\rho^{\bmod }(z)$ that is normalized at the origin by the experimental value of $f_{\pi}$, i.e., $\rho^{\bmod }(0)=2 \kappa_{\pi}^{2}$, but which is also able to reproduce the experimental value of the pion charge radius.

Evidently, to increase the radius, we should take a density which is larger for large $z$ than $\Phi^{2}\left(z, \kappa=\pi f_{\pi}\right)$. Since the overall integral normalization of the density is kept fixed, this can be achieved only by decreasing the density for small $z$ values.

Consider a simple ansatz (see Fig.4.8)

$$
\rho^{\bmod }(z)=2 \kappa_{\pi}^{2} e^{-z^{2} \kappa_{\rho}^{2}}\left[1-A z^{2} \kappa_{\rho}^{2}+B z^{4} \kappa_{\rho}^{4}\right],
$$

with $A=1-\kappa_{\rho}^{2} / \kappa_{\pi}^{2}+2 B$. It has both the desired value for $z=0$ and satisfies the normalization condition

$$
\int_{0}^{\infty} d z z \rho^{\bmod }(z)=1
$$

Integrating it with $\mathcal{J}^{\mathrm{O}}(Q, z)$ taken at $\kappa=\kappa_{\rho}$ produces the model form factor given by the following sum of contributions of the three lowest vector states:

$$
F_{\pi}^{\bmod }\left(Q^{2}\right)=\frac{2-(1-2 B) s_{0} / m_{\rho}^{2}}{1+Q^{2} / m_{\rho}^{2}}-\frac{1-(1-4 B) s_{0} / m_{\rho}^{2}}{1+Q^{2} / 2 m_{\rho}^{2}}+\frac{2 B s_{0} / m_{\rho}^{2}}{1+Q^{2} / 3 m_{\rho}^{2}} .
$$

The slope of $F_{\pi}^{\bmod }\left(Q^{2}\right)$ at $Q^{2}=0$ is given by

$$
\frac{d F_{\pi}^{\bmod }\left(Q^{2}\right)}{d Q^{2}}=-\frac{1}{m_{\rho}^{2}}\left[\frac{3}{2}-\left(\frac{1}{2}-\frac{2}{3} B\right) \frac{s_{0}}{m_{\rho}^{2}}\right] .
$$



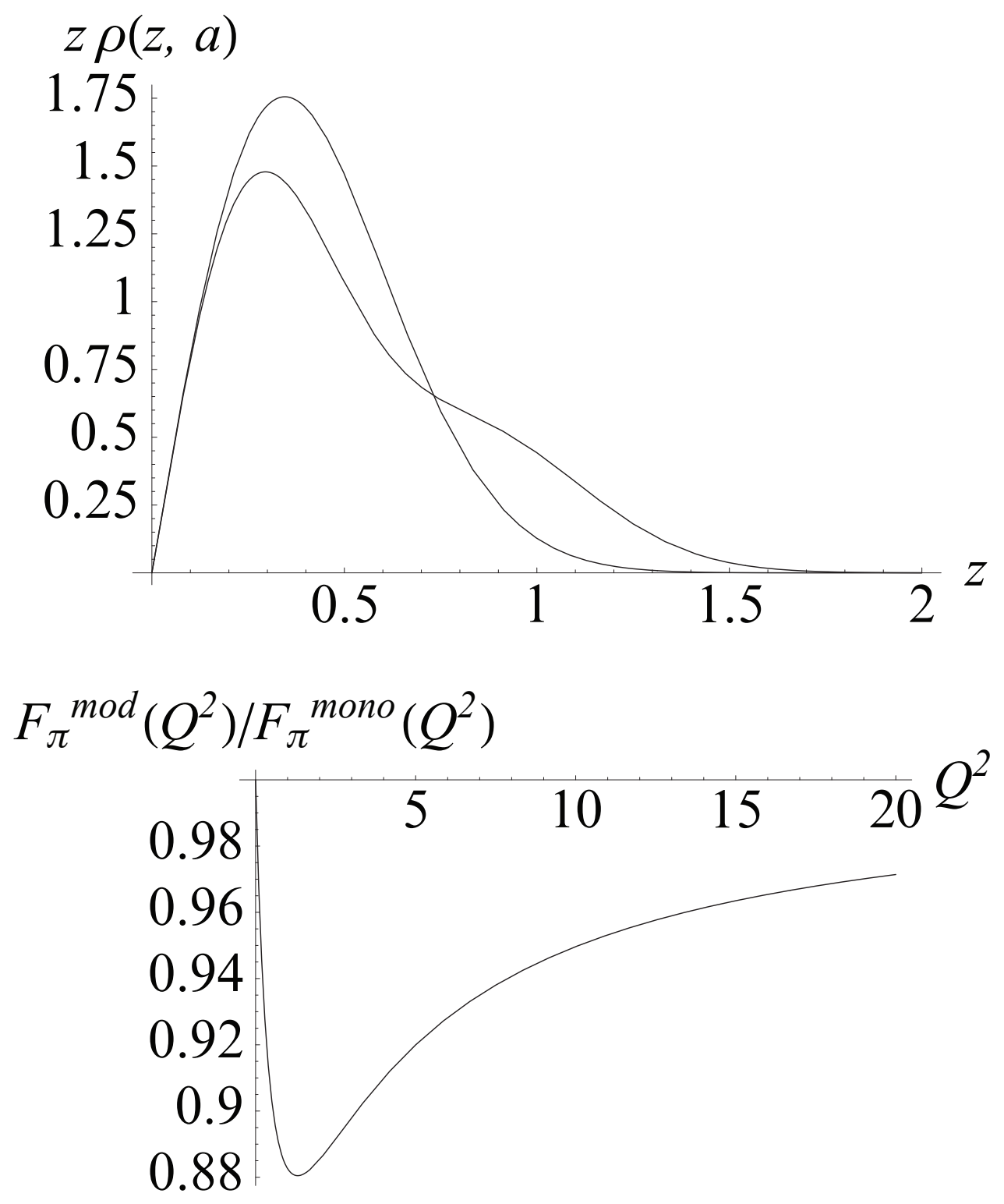

Figure 4.8: Top: Model density $z \rho^{\bmod }(z)$ (measured in $\mathrm{fm}^{-1}$ ) is larger than the density $z\left|\Phi\left(z, \kappa_{\pi}\right)\right|^{2}$ for large $z$ (displayed in fm). Bottom: Ratio $F_{\pi}^{\bmod }\left(Q^{2}\right) / F_{\pi}^{\operatorname{mono}}\left(Q^{2}\right)$ for $B=1 / 4$. 


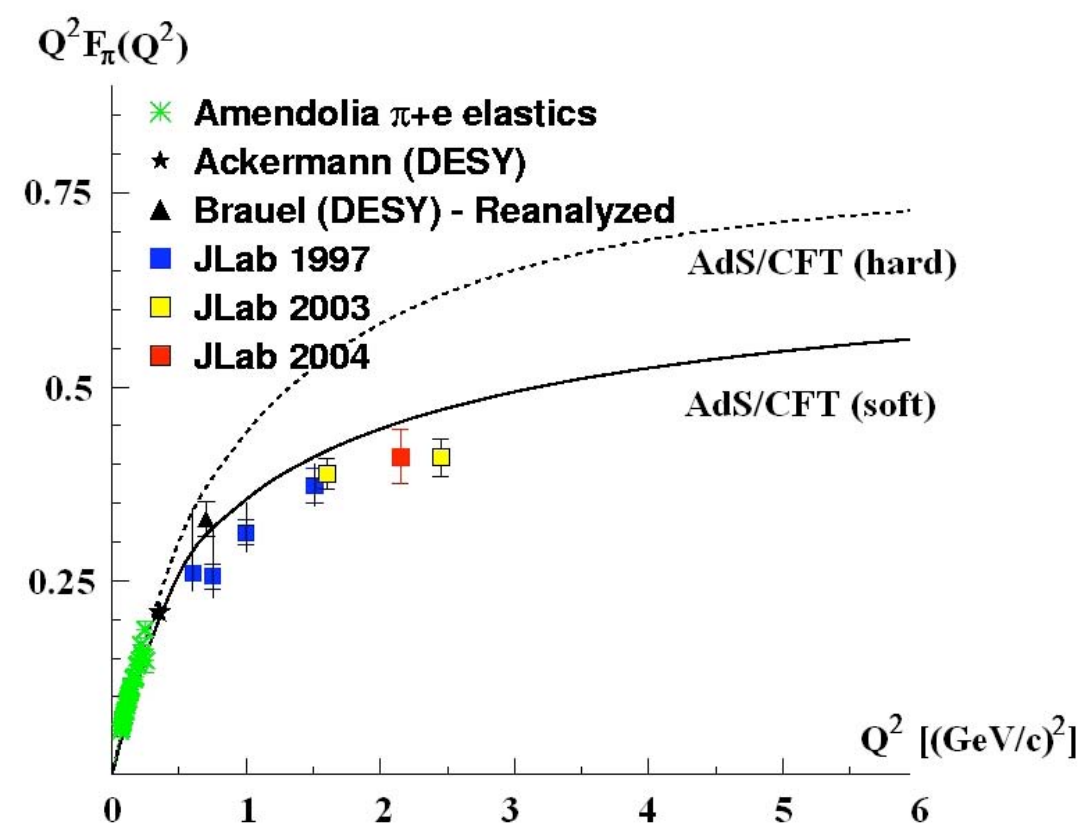

Figure 4.9: Experimental results for the pion form factor $Q^{2} F_{\pi}\left(Q^{2}\right)$ compared with hard-wall holographic model and the model with soft-wall like ansatz (figure edited using the JLab resources).

Taking $B=1 / 4$, one obtains the experimental value $0.45 \mathrm{fm}^{2}$ for $\left\langle r_{\pi}^{2}\right\rangle$. It is interesting to note that the model density providing this value, has an enhancement for larger values of $z$ (see Fig.4.8), just like the pion densities in the hard-wall model (see Fig.(4.4)). Due to a larger slope, $F_{\pi}^{\bmod }\left(Q^{2}\right)$ decreases faster than the simple monopole interpolation $F_{\pi}^{\text {mono }}\left(Q^{2}\right)$ and, as a result, is in better agreement with the data. In fact, it goes very close to $Q^{2} \lesssim 1 \mathrm{GeV}^{2}$ data, but exceeds the values of the JLab $Q^{2}=1.6$ and $2.45 \mathrm{GeV}^{2}$ points by roughly $10 \%$ and $20 \%$, respectively.

This discrepancy has a general reason. The asymptotic AdS/QCD prediction is $\left.Q^{2} F_{\pi}\left(Q^{2}\right)\right|_{Q^{2} \rightarrow \infty} \rightarrow 4 \pi^{2} f_{\pi}^{2}$ which is $\approx 0.68 \mathrm{GeV}^{2}$ for experimental value of $f_{\pi}$. On the other hand, JLab experimental points correspond to $Q^{2} F_{\pi}^{\exp }\left(Q^{2}\right) \approx 0.4 \mathrm{GeV}^{2}$, which is much smaller than the theoretical value quoted above. The pre-asymptotic effects, as we have seen, reduce the discrepancy, but there still remains a sizable gap. As we already stated, such a disagreement may be just a signal that we are reaching a region where AdS/QCD models should not be expected to work. In particular, AdS/QCD models of Refs. [23, 24, 26, 28] describe the pion in terms of an effective field or current, without specifying whether the current is built from spin- $1 / 2$ fields, or from scalar fields, etc. For $Q^{2}$ above $1 \mathrm{GeV}^{2}$, the quark substructure of the pion may be resolved by the electromagnetic probe (which is a wide-spread belief), and the description of the pion "as a whole" may be insufficient. 


\subsection{Summary}

In this chapter, we studied the pion in the chiral limit of two flavor QCD. To this end, we described a formalism that allows us to extract the pion form factor within the framework of the holographic dual model of QCD with hard-wall cutoff. Following Ref. [26], we identified the pion with the longitudinal component of the axial-vector gauge field. We defined two (Sturm-Liouville) conjugate wave functions $\Phi(z)$ and $\Psi(z)$ that describe the structure of the pion along the $5^{\text {th }}$ dimension coordinate $z$. These wave functions provide a very convenient framework to study the holographic physics of the pion. We demonstrated that, just like in the $\rho$-meson case [36], the pion form factor is given by an integral involving the function $\rho(z)$ that has the meaning of the charge density inside the pion. However, in contrast to the $\rho$-case, where the density was simply given by $|\Phi(z)|^{2}$, the pion density has an additional term proportional to $|\Psi(z)|^{2}$ and entering with a $z$-dependent coefficient reflecting the mechanism of the spontaneous symmetry breaking. Both terms are required for normalization of the form factor at $Q^{2}=0$.

We found an analytic expression for the pion decay constant in terms of two parameters of the model: $\sigma$ and $z_{0}$, similar to those used in Ref. [28]. Analyzing the results, we found it convenient to work with two combinations $\alpha=g_{5} \sigma / 3$ and $a=\alpha z_{0}^{3}$ of the basic parameters. In particular, we found $a=a_{0}=2.26$ for the value of $a$ corresponding to the experimental $\rho$-meson mass $m_{\rho}$ and pion decay constant $f_{\pi}$. The importance of the parameter $a$ is that its magnitude determines the regions where the pion properties are either governed by the confinement effects or by the effects from the spontaneous chiral symmetry breaking. For example, in the practically important domain $a>2$, the pion decay constant is determined primarily by $\sigma$, with negligibly tiny corrections due to the value of $z_{0}$. However, when $a<1$ the pion decay constant is proportional to the ratio $a / z_{0}$. For small $a \ll 1$, the radius of the pion is given by $\left\langle r_{\pi}^{2}\right\rangle=\frac{4}{3} z_{0}^{2}$, i.e., as one may expect, the pion size is completely determined by the confinement radius. On the other hand, for $a>2$ the radius is basically determined by $1 / \sigma^{1 / 3}$, slowly increasing with $z_{0}$ due to the $\ln a / a_{0}$ correction.

We also found that the pion rms charge radius $\left\langle r_{\pi}^{2}\right\rangle^{1 / 2} \approx 0.58 \mathrm{fm}$ in the hardwall model is smaller than that measured experimentally. In a sense, the hard wall at the distance $z_{0} \approx 0.62 \mathrm{fm}$ (fixed from the $\rho$-meson mass), "does not allow" the pion to get larger. So, we argued that if the IR wall is "softened", the size of the pion may be increased by an amount sufficient to accommodate the data. A straightforward idea is to use the soft-wall model of Ref. [32] and treat the pion in a way similar to what was done in [37] for the $\rho$-meson case. Unfortunately, there are prohibiting complications with directly introducing the chiral symmetry effects within the AdS/QCD model with the $z^{2}$ soft wall. As explained in Ref. [32], the chiral condensate $\sigma$ in such a model is proportional to the mass parameter $m_{q}$, so that in the chiral limit the condensate vanishes together with the quark mass.

To illustrate a possible change in the form factor predictions due to the softening of the IR wall, we proposed an ansatz for the pion density function and used the vector current source from the soft-wall model considered in Ref. [37]. We 
demonstrated that this ansatz is capable of giving the experimental value of the pion charge radius. It also closely follows the data in the $Q^{2}<1 \mathrm{GeV}^{2}$ region, while still overshoots available data in the $Q^{2} \sim 2 \mathrm{GeV}^{2}$ region. The basic source of this discrepancy is very general: the asymptotic AdS/QCD prediction for the pion form factor is $Q^{2} F_{\pi}\left(Q^{2}\right) \rightarrow 4 \pi^{2} f_{\pi}^{2}$, and if one takes the experimental value for $f_{\pi}$, one obtains $Q^{2} F_{\pi}\left(Q^{2}\right) \rightarrow 0.68 \mathrm{GeV}^{2}$, which is much larger than the $0.4 \mathrm{GeV}^{2}$ value given by $Q^{2} \sim 2 \mathrm{GeV}^{2}$ JLab data. For this reason, we argued that the disagreement mentioned above may be a signal that the region $Q^{2} \gtrsim 2 \mathrm{GeV}^{2}$ is beyond the applicability region of AdS/QCD models. 


\section{Chapter 5}

\section{Anomalous Form Factor of Pion in AdS/QCD Model}

\subsection{Introduction}

The form factor $F_{\gamma^{*} \gamma^{*} \pi^{0}}\left(Q_{1}^{2}, Q_{2}^{2}\right)$ describing the coupling of two (in general, virtual) photons with the lightest hadron, the pion, plays a special role in the studies of exclusive processes in quantum chromodynamics (QCD). When both photons are real, the form factor $F_{\gamma^{*} \gamma^{*} \pi^{0}}(0,0)$ determines the rate of the $\pi^{0} \rightarrow \gamma \gamma$ decay, and its value at this point is deeply related to the axial anomaly [67]. Because of this relation, the $\gamma^{*} \gamma^{*} \pi^{0}$ form factor has been an object of intensive studies since the 60's [68]-[72].

At large photon virtualities, its behavior was studied $[73,74,75]$ within a perturbative QCD (pQCD) factorization approach for exclusive processes [76, 77, 73]. Since only one hadron is involved, the $\gamma^{*} \gamma^{*} \pi^{0}$ form factor has the simplest structure for pQCD analysis, with the nonperturbative information about the pion accumulated in the pion distribution amplitude $\varphi_{\pi}(x)$ introduced in Refs. [78, 79]. Another simplification is that the short-distance amplitude for the $\gamma^{*} \gamma^{*} \pi^{0}$ vertex is given, at the leading order, just by a single quark propagator. Theoretically, the most clean situation is when both photon virtualities are large, but the experimental study of $F_{\gamma^{*} \gamma^{*} \pi^{0}}\left(Q_{1}^{2}, Q_{2}^{2}\right)$ in this regime through the $\gamma^{*} \gamma^{*} \rightarrow \pi^{0}$ process is very difficult due to a very small cross section.

The leading-twist pQCD factorization, however, works even if one of the photons is real or almost real. Furthermore, this kinematics is amenable to experimental investigation through the $\gamma \gamma^{*} \rightarrow \pi^{0}$ process at $e^{+} e^{-}$colliders. Comparison of the data obtained by the CELLO [80] and CLEO [81] collaborations with the original leading $[73,74]$ and next-to-leading order $[82,83,84,85]$ pQCD predictions amended by later studies $[86,87]$ that incorporate a more thorough treatment of the real photon channel using the light-cone QCD sum rule ideas provided important information about the shape of the pion distribution amplitude $\varphi_{\pi}(x)$ (for the most recent review see [88]). The momentum dependence of the $F_{\gamma^{*} \gamma^{*} \pi^{0}}\left(0, Q^{2}\right)$ form factor was also studied in various models of nonperturbative QCD dynamics (see, e.g., 
[89]-[102] and references therein).

In the present chapter*, our goal is to extend the holographic dual model of QCD to incorporate the anomalous $F_{\gamma^{*} \gamma^{*} \pi^{0}}\left(Q_{1}^{2}, Q_{2}^{2}\right)$ form factor. During the last few years applications of gauge/gravity duality [4] to hadronic physics attracted a lot of attention, and various holographic dual models of QCD were proposed in the literature (see, e.g., [23]-[111]). These models were able to incorporate such essential properties of QCD as confinement and dynamical chiral symmetry breaking, and also to reproduce many of the static hadronic observables (decay constants, masses), with values rather close to the experimental ones.

As the basis for our extension, we follow the holographic approach of Refs. [26, $28]$, and then intend to proceed along the lines of formalism outlined in our recent papers [36, 38], where it was first applied to form factors and wave functions of vector mesons [36, 39] (tensor form factors of vector mesons were considered in [112]) and later [38] to the pion electromagnetic form factor. However, a straightforward application of the approach of Refs. $[26,28,36,38]$ to the $F_{\gamma^{*} \gamma^{*} \pi^{0}}\left(Q_{1}^{2}, Q_{2}^{2}\right)$ form factor gives a vanishing result. There are two obvious reasons for such an outcome.

First, the five-dimensional (5D) gauge fields $B^{a}(x, z)$ in the AdS/QCD lagrangian of Refs. $[26,28]$ are only dual to the $4 \mathrm{D}$ isovector currents $J^{a}(x)$. On the other hand, a nonzero result for the matrix element $\left\langle 0\left|J_{\mathrm{EM}}^{\mu} J_{\mathrm{EM}}^{\nu}\right| \pi^{0}\right\rangle$ defining the $F_{\gamma^{*} \gamma^{*} \pi^{0}}\left(Q_{1}^{2}, Q_{2}^{2}\right)$ form factor may be obtained only when the electromagnetic currents $J_{\mathrm{EM}}^{\mu}, J_{\mathrm{EM}}^{\nu}$ have both isovector and isoscalar components, which is the case in two-flavor QCD, but not in the holographic models of Refs. [26, 28].

Thus, we need an AdS/QCD model that includes gauge fields in the 5D bulk which are dual to both isovector and isoscalar currents. The natural way to do this is to extend the gauge group $S U(2)_{L} \otimes S U(2)_{R}$ in the bulk up to $U(2)_{L} \otimes U(2)_{R}$. After explicit separation of the isosinglet and isovector parts, the new $5 \mathrm{D}$ field can be written as

$$
\mathcal{B}_{\mu}=t^{a} B_{\mu}^{a}+\mathbf{1} \frac{\hat{B}_{\mu}}{2}
$$

with 1 being the unity matrix. The $\hat{B}$ part is dual to $4 \mathrm{D}$ isosinglet vector current. Even after this modification, the AdS/QCD action gives zero result for the correlator $\left\langle 0\left|J_{\mu}^{\{I=1\}} J_{\nu}^{\{I=0\}} J_{\alpha}^{A}\right| 0\right\rangle$ involving two vector currents $J_{\mu}^{\{I=1\}}, J_{\nu}^{\{I=0\}}$ and the axial current $J_{\alpha}^{A}$ (that has nonzero projection onto the pion state). To bring the anomalous amplitude into the model, the next step (similar to [114]) is to add a Chern-Simons term [115] to the action. After these extensions, the calculation of the $F_{\gamma^{*} \gamma^{*} \pi^{0}}\left(Q_{1}^{2}, Q_{2}^{2}\right)$ form factor may be performed using the methods developed in Refs. [36, 38].

To make this section clear by itself, we start by recalling the basics of the hardwall model, in particular, the form of the action given in Ref. [26] and our results [38] for the pion wave function. We consider the generalization of the AdS/QCD model that includes isoscalar fields and Chern-Simons term. Using this extended model we describe the calculation of the $F_{\gamma^{*} \gamma^{*} \pi^{0}}\left(Q_{1}^{2}, Q_{2}^{2}\right)$ form factor and express

*The main results from the Ref. [40] are printed by permission from the APS, see Appendix. 
it in terms of the pion wave function and two bulk-to-boundary propagators for the vector currents describing EM sources. We study the results obtained within the extended AdS/QCD model with one real and one slightly virtual photon and calculate the value of the $Q^{2}$-slope of the form factor. We also discuss the formal limit of large photon virtualities, and compare these results to those obtained in pQCD. Furthermore, we study the generalized VMD structure of the AdS/QCD model expression for the $F_{\gamma^{*} \gamma^{*} \pi^{0}}\left(Q_{1}^{2}, Q_{2}^{2}\right)$ form factor. Finally, we conclude this chapter by summary.

\subsection{Overview}

In the holographic model, QCD resonances correspond to Kaluza-Klein type excitations of the sliced $\mathrm{AdS}_{5}$ space. The basic prescription is that there is a correspondence between the 4D vector and axial-vector currents and 5D gauge fields $V_{\mu}^{a}(x, z)$ and $A_{\mu}^{a}(x, z)$. Furthermore, since the gauge invariance corresponding to the axial-vector current is spontaneously broken in the 5D background, the longitudinal component of the axial-vector field becomes physical and related to the pion field.

\subsubsection{AdS/QCD Action}

The action of the holographic model of Ref. [26] can be written in the form

$$
\begin{aligned}
S_{\mathrm{AdS}}^{B} & =\operatorname{Tr} \int d^{4} x \int_{0}^{z_{0}} d z\left[\frac{1}{z^{3}}\left(D^{M} X\right)^{\dagger}\left(D_{M} X\right)+\frac{3}{z^{5}} X^{\dagger} X\right. \\
& \left.-\frac{1}{8 g_{5}^{2} z}\left(B_{(L)}^{M N} B_{(L) M N}+B_{(R)}^{M N} B_{(R) M N}\right)\right],
\end{aligned}
$$

where $D X=\partial X-i B_{(L)} X+i X B_{(R)},\left(B_{(L, R)}=V \pm A\right)$ and $X(x, z)=v(z) U(x, z) / 2$ is taken as a product of the chiral field $U(x, z)=\exp \left[2 i t^{a} \pi^{a}(x, z)\right]$ (as usual, $t^{a}=\sigma^{a} / 2$, with $\sigma^{a}$ being Pauli matrices) and the function $v(z)=\left(m_{q} z+\sigma z^{3}\right)$ containing the chiral symmetry breaking parameters $m_{q}$ and $\sigma$, with $m_{q}$ playing the role of the quark mass and $\sigma$ that of the quark condensate.

In general, one can write $A=A_{\perp}+A_{\|}$, where $A_{\perp}$ and $A_{\|}$are transverse and longitudinal components of the axial-vector field. The spontaneous symmetry breaking causes $A_{\|}$to be physical and associated with the Goldstone boson, the pion in this case. The longitudinal component is written in the form:

$$
A_{\| M}^{a}(x, z)=\partial_{M} \psi^{a}(x, z) .
$$

Then $\psi^{a}(x, z)$ corresponds to the pion field. This Higgs-like mechanism breaks the axial-vector gauge invariance by bringing a $z$-dependent mass term into the $A$-part of the lagrangian.

Varying the action with respect to the transverse gauge fields $V_{\mu}^{a}$ and $A_{\perp \mu}^{a}$ gives

equations of motion for these fields describing (via the holographic correspondence) 
the physics of vector and axial-vector mesons. Variation with respect to $A_{\| \mu}^{a}$ and $A_{z}^{a}$ gives two coupled equations for the chiral field $\pi^{a}(x, z)$ and the pion field $\psi^{a}(x, z)$. It is convenient to work in Fourier representation, where $\tilde{V}_{\mu}^{a}(p, z)=\tilde{V}_{\mu}^{a}(p) \mathcal{V}(p, z)$ is the Fourier transform of $V_{\mu}^{a}(x, z)$, and $\tilde{A}_{\mu}^{a}(p, z)$ is the Fourier transform of $A_{\mu}^{a}(x, z)$.

\subsubsection{Vector Channel}

The vector bulk-to-boundary propagator

$$
\mathcal{V}(p, z)=g_{5} \sum_{m=1}^{\infty} \frac{f_{m} \psi_{m}^{V}(z)}{-p^{2}+M_{m}^{2}}
$$

has the bound-state poles for $p^{2}=M_{n}^{2}$, with the resonance masses $M_{n}=\gamma_{0, n} / z_{0}$ $\left(\gamma_{0, n}\right.$ is $n^{\text {th }}$ zero of the Bessel function $\left.J_{0}(x)\right)$ determined by the eigenvalues of the equation of motion

$$
\partial_{z}\left[\frac{1}{z} \partial_{z} \psi_{n}^{V}(z)\right]+\frac{1}{z} M_{n}^{2} \psi_{n}^{V}(z)=0
$$

subject to boundary conditions $\psi_{n}^{V}(0)=\partial_{z} \psi_{n}^{V}\left(z_{0}\right)=0$. The eigenfunctions of this equation give the " $\psi$ " wave functions

$$
\psi_{n}^{V}(z)=\frac{\sqrt{2}}{z_{0} J_{1}\left(\gamma_{0, n}\right)} z J_{1}\left(M_{n} z\right)
$$

for the relevant resonances. The coupling constants $f_{n}$ are determined from the $\psi$ wave functions through

$$
f_{n}=\frac{1}{g_{5}}\left[\frac{1}{z} \partial_{z} \psi_{n}^{V}(z)\right]_{z=0}=\frac{\sqrt{2} M_{n}}{g_{5} z_{0} J_{1}\left(\gamma_{0, n}\right)} .
$$

In Ref. [36], we introduced " $\phi$ wave functions"

$$
\phi_{n}^{V}(z) \equiv \frac{1}{M_{n} z} \partial_{z} \psi_{n}^{V}(z)=\frac{\sqrt{2}}{z_{0} J_{1}\left(\gamma_{0, n}\right)} J_{0}\left(M_{n} z\right),
$$

which give the couplings $g_{5} f_{n} / M_{n}$ as their values at the origin, just like the $(L=0)$ bound state wave functions in quantum mechanics. Moreover, these functions satisfy Dirichlet b.c. $\phi_{n}^{V}\left(z_{0}\right)=0$. Physically, the $\psi^{V}$ wave functions describe the vector bound states in terms of the vector potential $V_{\mu}$, while the $\phi^{V}$ wave functions describe them in terms of the field-strength tensor $\partial_{z} V_{\mu}=V_{z \mu}$ (we work in the axial gauge, where $\left.V_{z}=0\right)$.

An essential ingredient of form factor formulas is the vector bulk-to-boundary propagator $\mathcal{J}(Q, z) \equiv \mathcal{V}(i Q, z)$ taken at a spacelike momentum $p$ with $p^{2}=-Q^{2}$. It can be written in a closed form as

$$
\mathcal{J}(Q, z)=Q z\left[K_{1}(Q z)+I_{1}(Q z) \frac{K_{0}\left(Q z_{0}\right)}{I_{0}\left(Q z_{0}\right)}\right] .
$$

The function $\mathcal{J}(Q, z)$ satisfies the relations $\mathcal{J}(Q, 0)=1, \mathcal{J}(0, z)=1$ and $\partial_{z} \mathcal{J}\left(Q, z_{0}\right)=$ 0 . 


\subsubsection{Pion Channel}

An important achievement of the hard-wall model of Ref. [26] is its compliance with the Gell-Mann-Oakes-Renner relation $m_{\pi}^{2} \sim m_{q}$ that produces a massless pion in the $m_{q}=0$ limit. The fits of Ref. [26] give a very small value $m_{q} \sim 2 \mathrm{MeV}$ for the "quark mass" parameter $m_{q}$, so it makes sense to resort to the chiral $m_{q}=0$ limit, which has an additional advantage that solutions of the equations of motion in this case can be found analytically. The pion wave function $\psi(z)$ is introduced through the longitudinal part of the axial-vector field:

$$
\tilde{A}_{\| \mu}^{a}(p, z)=\frac{p_{\mu} p^{\alpha}}{p^{2}} \tilde{A}_{\alpha}^{a}(p) \psi(z)
$$

The bulk-to-boundary part $\pi(z)$ of the $\pi^{a}(z)$ field in the zeroth order of the of $m_{\pi}^{2}$ expansion proposed in Ref [26] tends to -1 . Then the equation for $\Psi(z) \equiv$ $\psi(z)-\pi(z)$ is exactly solvable, with the result

$$
\Psi(z)=z \Gamma(2 / 3)\left(\frac{\alpha}{2}\right)^{1 / 3}\left[I_{-1 / 3}\left(\alpha z^{3}\right)-I_{1 / 3}\left(\alpha z^{3}\right) \frac{I_{2 / 3}\left(\alpha z_{0}^{3}\right)}{I_{-2 / 3}\left(\alpha z_{0}^{3}\right)}\right],
$$

where $\alpha=g_{5} \sigma / 3$ (one may use here Airy functions instead of $I_{-1 / 3}(x), I_{2 / 3}(x)$, cf. [59]). The pion wave function $\Psi(z)$ coincides with the axial-vector bulk-to-boundary propagator $\mathcal{A}(0, z)$ taken at $p^{2}=0$. It is normalized by $\Psi(0)=1$, satisfies Neumann boundary condition $\Psi^{\prime}\left(z_{0}\right)=0$ at the IR boundary and, due to the holographic correspondence, has the property that

$$
f_{\pi}^{2}=-\frac{1}{g_{5}^{2}}\left(\frac{1}{z} \partial_{z} \Psi(z)\right)_{z=\epsilon \rightarrow 0}
$$

For the neutral pion, it is convenient to define $f_{\pi}$ through the matrix element of the $\sigma_{3}$ projection of the axial-vector current

$$
\left\langle 0\left|J_{\mu}^{A, 3}\right| \pi^{0}(p)\right\rangle \equiv\left\langle 0\left|\frac{\bar{u} \gamma_{\mu} \gamma_{5} u-\bar{d} \gamma_{\mu} \gamma_{5} d}{2}\right| \pi^{0}(p)\right\rangle=i f_{\pi} p_{\mu} .
$$

Then, the (experimental) numerical value of $f_{\pi}$ is $92.4 \mathrm{MeV}$. Matching $\mathrm{AdS}_{5}$ result $\Sigma^{\mathrm{AdS}}\left(p^{2}\right) \sim \ln p^{2} /\left(2 g_{5}^{2}\right)$ and QCD result $\Sigma_{3}^{\mathrm{QCD}}\left(p^{2}\right) \sim \ln p^{2} /\left(8 \pi^{2}\right)$ for the large- $p^{2}$ behavior of the correlator of $J_{\mu}^{A, 3}$ currents gives $g_{5}=2 \pi$ [26]. Analyzing the pion EM form factor, one deals with charged pions, and the choice of the axial-vector current as $J_{\mu}^{A,(\mathrm{c})}=\bar{d} \gamma_{\mu} \gamma_{5} u$ is more natural. Then $f_{\pi}^{(\mathrm{c})}=130.7 \mathrm{MeV}$, while $\Sigma_{(\mathrm{c})}^{\mathrm{QCD}}\left(p^{2}\right) \sim$ $\ln p^{2} /\left(4 \pi^{2}\right)$, hence, $g_{5}^{(\mathrm{c})}=\sqrt{2} \pi$ [37]. Of course, the combination $g_{5}^{2} f_{\pi}^{2}$, being the ratio of the coefficient of the pion pole contribution $\Sigma_{\pi}\left(p^{2}\right) \sim f_{\pi}^{2} / p^{2}$ to the coefficient $\sim 1 / g_{5}^{2}$ in $\Sigma\left(p^{2}\right)$ 's large- $p^{2}$ behavior, remains intact:

$$
g_{5}^{2} f_{\pi}^{2}=4 \pi^{2} f_{\pi}^{2}=2 \pi^{2}\left(f_{\pi}^{(c)}\right)^{2}=\left(g_{5}^{(\mathrm{c})} f_{\pi}^{(\mathrm{c})}\right)^{2} \equiv s_{0} / 2,
$$

where $s_{0} \approx 0.67 \mathrm{GeV}^{2}$. It is this convention-independent combination that enters Eq. (5.11). 
Again, it is convenient to introduce the conjugate wave function [38]:

$$
\Phi(z)=-\frac{1}{g_{5}^{2} f_{\pi}^{2}}\left(\frac{1}{z} \partial_{z} \Psi(z)\right)=-\frac{2}{s_{0}}\left(\frac{1}{z} \partial_{z} \Psi(z)\right) .
$$

It vanishes at the IR boundary $z=z_{0}$, i.e., $\Phi\left(z_{0}\right)=0$ and, according to Eq. (5.11), is normalized as $\Phi(0)=1$ at the origin. From $\Phi(0)=1$, it follows that the pion decay constant can be written as a function

$$
g_{5}^{2} f_{\pi}^{2}=3 \cdot 2^{1 / 3} \frac{\Gamma(2 / 3)}{\Gamma(1 / 3)} \frac{I_{2 / 3}\left(\alpha z_{0}^{3}\right)}{I_{-2 / 3}\left(\alpha z_{0}^{3}\right)} \alpha^{2 / 3}
$$

of the condensate parameter $\alpha$ and the confinement radius $z_{0}$. Note that the magnitude of $\alpha$ is independent of the $g_{5}$-convention, while the value of $\sigma$ depends on the $g_{5}$-convention used.

After fixing $z_{0}$ through the $\rho$-meson mass, $z_{0}=z_{0}^{\rho}=(323 \mathrm{MeV})^{-1}$, the experimental $f_{\pi}$ is obtained for $\alpha=(424 \mathrm{MeV})^{3}$. For these values, the argument $a \equiv \alpha z_{0}^{3}$ of the modified Bessel functions in Eq. (5.15) equals $2.26 \equiv a_{0}$. Since $I_{2 / 3}(a) / I_{-2 / 3}(a) \approx$ 1 for $a \gtrsim 1$, then

$$
g_{5}^{2} f_{\pi}^{2} \approx 3 \cdot 2^{1 / 3} \frac{\Gamma(2 / 3)}{\Gamma(1 / 3)} \alpha^{2 / 3},
$$

i.e., the value of $f_{\pi}$ is basically determined by $\alpha$ alone (the same observation was made in the pioneering paper [28] and in a recent paper [59] in which the pion channel was studied numerically).

In Fig. 5.1, we illustrate the behavior of the pion wave functions $\Psi$ and $\Phi$ representing them $\Psi \rightarrow \psi(\zeta, a), \Phi \rightarrow \varphi(\zeta, a)$ as functions of dimensionless variables $\zeta \equiv z / z_{0}$ and $a \equiv \alpha z_{0}^{3}$. For $a=0$ (i.e. when the chiral symmetry breaking parameter $\alpha$ vanishes), the limiting forms are $\psi(\zeta, 0)=1$ and $\varphi(\zeta, 0)=1-\zeta^{4}$ [38]. As $a$ increases, both functions become more and more narrow, with $\psi(\zeta, a)$ becoming smaller and smaller at the IR boundary $\zeta=1$.

\subsection{Anomalous Amplitude}

\subsubsection{Isosinglet Fields}

The $\pi^{0} \gamma^{*} \gamma^{*}$ form factor is defined by

$$
\int\left\langle\pi, p\left|T\left\{J_{\mathrm{EM}}^{\mu}(x) J_{\mathrm{EM}}^{\nu}(0)\right\}\right| 0\right\rangle e^{-i q_{1} x} d^{4} x=\epsilon^{\mu \nu \alpha \beta} q_{1 \alpha} q_{2 \beta} F_{\gamma^{*} \gamma^{*} \pi^{0}}\left(Q_{1}^{2}, Q_{2}^{2}\right),
$$

where $p=q_{1}+q_{2}$ and $q_{1,2}^{2}=-Q_{1,2}^{2}$. Its value for real photons

$$
F_{\gamma^{*} \gamma^{*} \pi^{0}}(0,0)=\frac{N_{c}}{12 \pi^{2} f_{\pi}}
$$



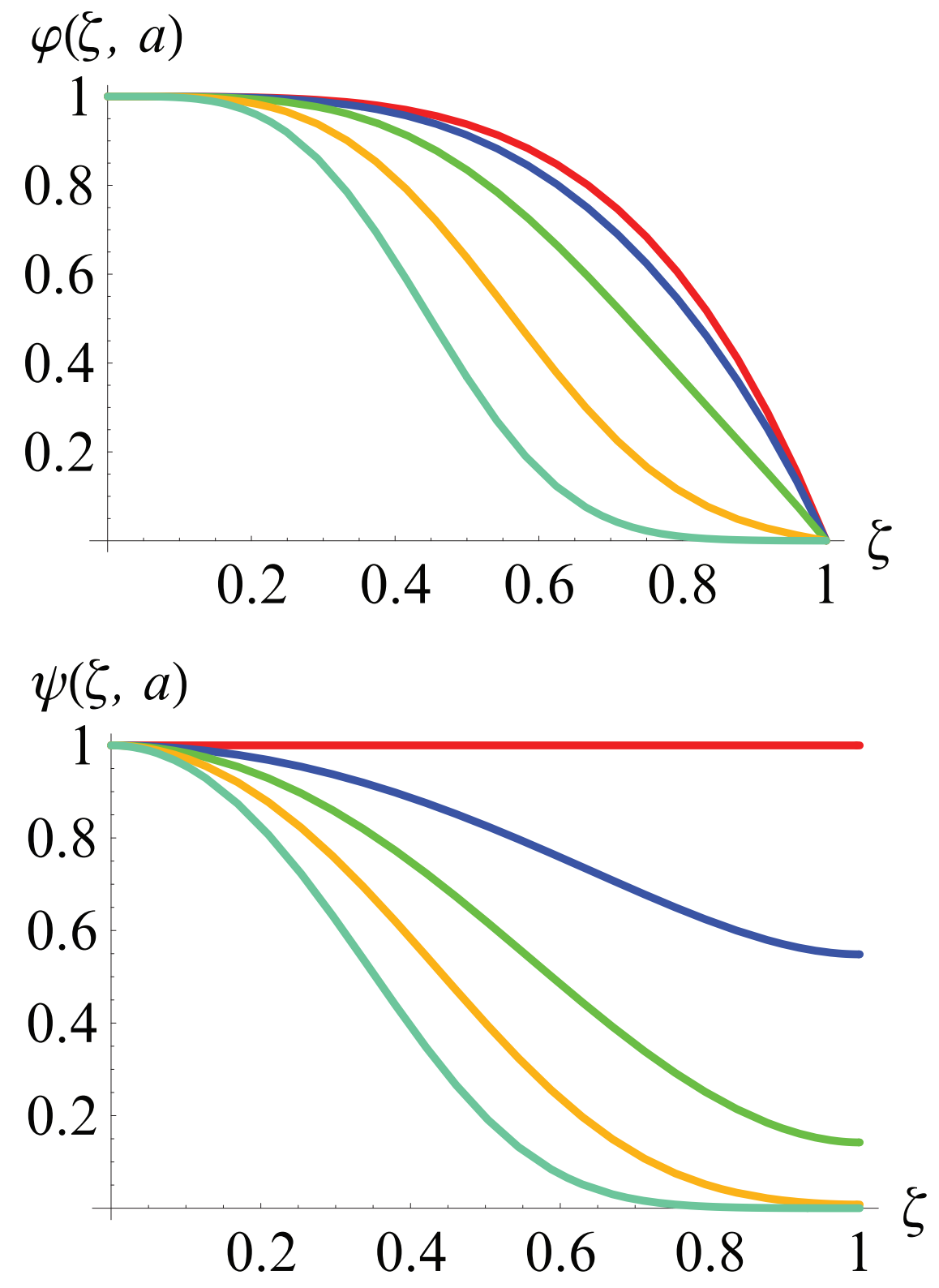

Figure 5.1: Functions $\psi(\zeta, a)$ (top) and $\varphi(\zeta, a)$ (bottom) for several values of $a$ : $a=0$ (uppermost lines), $a=1, a=2.26, a=5, a=10$ (lowermost lines). 
is related in QCD to the axial anomaly.

Since $\pi^{0}$ meson is described by the third component $A^{3}$ of an isovector field, only the isovector component of the product of two electromagnetic currents gives nonzero contribution to the matrix element in Eq. (5.17). In QCD, the electromagnetic current is given by the sum

$$
J_{\mu}^{\mathrm{EM}}=J_{\mu}^{\{I=1\}, 3}+\frac{1}{3} J_{\mu}^{\{I=0\}}
$$

of isovector $J_{\mu}^{\{I=1\}, a}(x)$ (" $\rho$-type") and isosinglet $J_{\mu}^{\{I=0\}}(x)$ (" $\omega$-type") currents,

$$
\begin{aligned}
& J_{\mu}^{\{I=1\}, 3}=\frac{1}{2}\left(\bar{u} \gamma_{\mu} u-\bar{d} \gamma_{\mu} d\right)=\bar{q} \gamma_{\mu} t^{3} q, \\
& J_{\mu}^{\{I=0\}}=\frac{1}{2}\left(\bar{u} \gamma_{\mu} u+\bar{d} \gamma_{\mu} d\right)=\frac{1}{2} \bar{q} \gamma_{\mu} \mathbf{1} q .
\end{aligned}
$$

As a result, the matrix element $\left\langle\pi^{0}\left|J^{\mathrm{EM}} J^{\mathrm{EM}}\right| 0\right\rangle$ is nonzero since

$$
\left\langle\pi^{0}\left|J^{\{I=1\}, 3} J^{\{I=0\}}\right| 0\right\rangle \sim \operatorname{Tr}\left(t^{3} t^{3}\right) .
$$

To extract $F_{\gamma^{*} \gamma^{*} \pi^{0}}\left(Q_{1}^{2}, Q_{2}^{2}\right)$ form factor via holographic correspondence, we consider the correlator of the axial-vector current

$$
J_{\mu}^{A, a}=\bar{q} \gamma_{\mu} \gamma_{5} t^{a} q
$$

and vector currents $J_{\mu}^{\{I=1\}, b}, J_{\nu}^{\{I=0\}}$. To proceed, we need to have isoscalar fields on the AdS side of the holographic correspondence. This is achieved by gauging $U(2)_{L} \otimes U(2)_{R}$ rather than $S U(2)_{L} \otimes S U(2)_{R}$ group in the AdS/QCD action, i.e., by substituting $t^{a} B_{\mu}^{a}$ by

$$
\mathcal{B}_{\mu}=t^{a} B_{\mu}^{a}+1 \frac{\hat{B}_{\mu}}{2}
$$

in Eq. (5.1). Then the 4D currents correspond to the following 5D gauge fields

$$
\begin{aligned}
J_{\mu}^{A, a}(x) & \rightarrow A_{\mu}^{a}(x, z), \\
J_{\mu}^{\{I=0\}}(x) & \rightarrow \hat{V}_{\mu}(x, z), \\
J_{\mu}^{\{I=1\}, a}(x) & \rightarrow V_{\mu}^{a}(x, z) .
\end{aligned}
$$

\subsubsection{Chern-Simons Term}

The terms contained in the original AdS/QCD action (5.1) cannot produce a 3-point function accompanied by the Levi-Civita $\epsilon^{\mu \nu \alpha \beta}$ factor. However, such a contribution may be obtained by adding the Chern-Simons (CS) term. We follow Refs. [113] in choosing the form of the $\mathcal{O}\left(B^{3}\right)$ part of the $D=5$ CS action (the only one we need). In the axial gauge $B_{z}=0$, it is written as

$$
S_{\mathrm{CS}}^{(3)}[\mathcal{B}]=k \frac{N_{c}}{48 \pi^{2}} \epsilon^{\mu \nu \rho \sigma} \operatorname{Tr} \int d^{4} x d z\left(\partial_{z} \mathcal{B}_{\mu}\right)\left[\mathcal{F}_{\nu \rho} \mathcal{B}_{\sigma}+\mathcal{B}_{\nu} \mathcal{F}_{\rho \sigma}\right]
$$


where $\mathcal{F}$ is the field-strength generated by $\mathcal{B}$ and $k$ should be an integer number adjusted to reproduce the QCD anomaly result. Then, in the $U(2)_{L} \otimes U(2)_{R}$ model, the relevant part of the CS term is [114]

$$
S_{\mathrm{CS}}^{\mathrm{AdS}}\left[\mathcal{B}_{L}, \mathcal{B}_{R}\right]=S_{\mathrm{CS}}^{(3)}\left[\mathcal{B}_{L}\right]-S_{\mathrm{CS}}^{(3)}\left[\mathcal{B}_{R}\right]
$$

Taking into account that $\mathcal{B}_{L, R}=\mathcal{V} \pm \mathcal{A}$, and keeping only the longitudinal component of the axial-vector field $A \rightarrow A_{\|}$(that brings in the pion), for which $F_{\mu \nu}^{A}=0$, we have

$$
\begin{aligned}
S_{\mathrm{CS}}^{\mathrm{AdS}} & =k \frac{N_{c}}{24 \pi^{2}} \epsilon^{\mu \nu \rho \sigma} \int d^{4} x \int_{0}^{z_{0}} d z \\
& \times\left[\left(\partial_{\rho} V_{\mu}^{a}\right)\left(A_{\| \sigma}^{a} \stackrel{\leftrightarrow}{\partial_{z}} \hat{V}_{\nu}\right)+\left(\partial_{\rho} \hat{V}_{\mu}\right)\left(A_{\| \sigma}^{a} \overleftrightarrow{\partial}_{z} V_{\nu}^{a}\right)\right],
\end{aligned}
$$

where $\stackrel{\leftrightarrow}{\partial_{z}} \equiv \vec{\partial}_{z}-\overleftarrow{\partial}_{z}$. Representing $A_{\| \sigma}^{a}=\partial_{\sigma} \psi^{a}$ and integrating by parts gives

$$
\begin{aligned}
S_{\mathrm{CS}}^{\mathrm{AdS}} & =k \frac{N_{c}}{24 \pi^{2}} \epsilon^{\mu \nu \rho \sigma} \int d^{4} x \int_{0}^{z_{0}} d z \\
& \times\left[2\left(\partial_{z} \psi^{a}\right)\left(\partial_{\rho} V_{\mu}^{a}\right)\left(\partial_{\sigma} \hat{V}_{\nu}\right)-\psi^{a} \partial_{z}\left(\partial_{\rho} V_{\mu}^{a} \partial_{\sigma} \hat{V}_{\nu}\right)\right] .
\end{aligned}
$$

Note that the $z$-derivative of $\psi^{a}$ is proportional to the $\Phi$-function of the pion (5.14), which, as we argued in Refs. [36, 37, 38], is the most direct analog of the quantummechanical bound state wave functions, i.e., it is the derivative $\partial_{z} \psi^{a}(z)$ rather than $\psi^{a}(z)$ itself that is an analog of the pion 4D field. Then, the first term in the square brackets in Eq. (5.27) has the structure similar to the $\pi \omega \rho$ interaction term

$$
\mathcal{L}_{\pi \omega \rho}=\frac{N_{c} g^{2}}{8 \pi^{2} f_{\pi}} \epsilon^{\mu \nu \alpha \beta} \pi^{a}\left(\partial_{\mu} \rho_{\nu}^{a}\right)\left(\partial_{\alpha} \omega_{\beta}\right)
$$

obtained in the hidden local symmetries approach [116] from the anomalous WessZumino $[117,118]$ lagrangian (see also a review [119]). Here $g$ is the universal gauge coupling constant of that approach.

Integrating by parts over $z$, the second term of Eq. (5.27) can be also converted into a contribution of this form plus a $z=z_{0}$ surface term:

$$
\begin{aligned}
S_{\mathrm{CS}}^{\mathrm{AdS}} & =k \frac{N_{c}}{24 \pi^{2}} \epsilon^{\mu \nu \rho \sigma} \int d^{4} x\left\{-\left.\left[\psi^{a}\left(\partial_{\rho} V_{\mu}^{a}\right)\left(\partial_{\sigma} \hat{V}_{\nu}\right)\right]\right|_{z=z_{0}}\right. \\
& \left.+3 \int_{0}^{z_{0}} d z\left(\partial_{z} \psi^{a}\right)\left(\partial_{\rho} V_{\mu}^{a}\right)\left(\partial_{\sigma} \hat{V}_{\nu}\right)\right\} .
\end{aligned}
$$

The latter can be eliminated by adding a compensating surface term into the original Chern-Simons action, so that the resulting anomalous part of the action in the extended AdS/QCD model

$$
S^{\mathrm{anom}}=k \frac{N_{c}}{8 \pi^{2}} \epsilon^{\mu \nu \rho \sigma} \int d^{4} x \int_{0}^{z_{0}} d z\left(\partial_{z} \psi^{a}\right)\left(\partial_{\rho} V_{\mu}^{a}\right)\left(\partial_{\sigma} \hat{V}_{\nu}\right)
$$

has the structure of Eq. (5.28). 


\subsubsection{Three-Point Function}

The action (5.29) produces the 3-point function

$$
\left\langle J_{\alpha}^{A, 3}(-p) J_{\mu}^{\mathrm{EM}}\left(q_{1}\right) J_{\nu}^{\mathrm{EM}}\left(q_{2}\right)\right\rangle=T_{\alpha \mu \nu}\left(p, q_{1}, q_{2}\right) i(2 \pi)^{4} \delta^{(4)}\left(q_{1}+q_{2}-p\right),
$$

with

$$
T_{\alpha \mu \nu}\left(p, q_{1}, q_{2}\right)=\frac{N_{c}}{12 \pi^{2}} \frac{p_{\alpha}}{p^{2}} \epsilon_{\mu \nu \rho \sigma} q_{1}^{\rho} q_{2}^{\sigma} K_{b}\left(Q_{1}^{2}, Q_{2}^{2}\right)
$$

Here, $p$ is the momentum of the pion and $q_{1}, q_{2}$ are the momenta of the photons.

The "bare" function $K_{b}\left(Q_{1}^{2}, Q_{2}^{2}\right)$ is given by

$$
K_{b}\left(Q_{1}^{2}, Q_{2}^{2}\right)=-\frac{k}{2} \int_{0}^{z_{0}} \mathcal{J}\left(Q_{1}, z\right) \mathcal{J}\left(Q_{2}, z\right) \partial_{z} \psi(z) d z
$$

where $\mathcal{J}\left(Q_{1}, z\right), \mathcal{J}\left(Q_{2}, z\right)$ are the vector bulk-to-boundary propagators (5.8), and $\psi(z)$ is the pion wave function (5.10).

\subsubsection{Conforming to QCD Axial Anomaly}

For real photons, i.e., when $Q_{1}^{2}=Q_{2}^{2}=0$, the value of the $F_{\pi \gamma^{*} \gamma^{*}}\left(Q_{1}^{2}, Q_{2}^{2}\right)$ form factor in QCD (with massless quarks) is settled by the axial anomaly, which corresponds to $K^{\mathrm{QCD}}(0,0)=1$. Our goal is to build an AdS/QCD model for $F_{\pi \gamma^{*} \gamma^{*}}\left(Q_{1}^{2}, Q_{2}^{2}\right)$ that reproduces this value. Taking $Q_{1}^{2}=Q_{2}^{2}=0$, we have $\mathcal{J}(0, z)=1$, and Eq. (5.32) gives

$$
K_{b}(0,0)=-\frac{k}{2} \int_{0}^{z_{0}} \partial_{z} \psi(z) d z=-\frac{k}{2} \psi\left(z_{0}\right)=\frac{k}{2}\left[1-\Psi\left(z_{0}\right)\right] .
$$

On the IR boundary $z=z_{0}$, the pion wave function $\Psi(z)$ from Eq. (5.10) is

$$
\Psi\left(z_{0}\right)=\frac{\sqrt{3} \Gamma(2 / 3)}{\pi I_{-2 / 3}(a)}\left(\frac{1}{2 a^{2}}\right)^{1 / 3} .
$$

As we discussed above, experimental values of $m_{\rho}$ and $f_{\pi}$ correspond to $a=2.26$, which gives $\Psi\left(z_{0}\right)=0.14$. The magnitude of $\Psi\left(z_{0}\right)$ rapidly decreases for larger $a$ (e.g., $\left.\Psi\left(z_{0}\right)\right|_{a=4} \approx 0.02$, see Fig. 5.1). Still, the value of $\Psi\left(z_{0}\right)$ is nonzero at any finite value of $a$, and it is impossible to exactly reproduce the anomaly result by simply adjusting the integer number $k$. To conform to the QCD anomaly value $K^{\mathrm{QCD}}(0,0)=1$, we add a surface term compensating the $\Psi\left(z_{0}\right)$ contribution in Eq. (34) and then take $k=2$. To fix the form of the surface term, we note first that it should have the structure of a $V V A 3$-point function taken on the $z=z_{0}$ surface.

Furthermore, using the derivatives $\mathcal{J}_{z}^{\prime}\left(Q_{i}, z_{0}\right)$ of the bulk-to-boundary propagators in this term is excluded, because $\mathcal{J}_{z}^{\prime}\left(Q_{i}=0, z_{0}\right)=0$ at the real photon point. On the 
other hand, $\mathcal{J}\left(Q_{i}=0, z_{0}\right)=1$, and our final model for $F_{\pi \gamma^{*} \gamma^{*}}\left(Q_{1}^{2}, Q_{2}^{2}\right)$ corresponds to the function

$$
\begin{aligned}
K\left(Q_{1}^{2}, Q_{2}^{2}\right) & =\Psi\left(z_{0}\right) \mathcal{J}\left(Q_{1}, z_{0}\right) \mathcal{J}\left(Q_{2}, z_{0}\right) \\
& -\int_{0}^{z_{0}} \mathcal{J}\left(Q_{1}, z\right) \mathcal{J}\left(Q_{2}, z\right) \partial_{z} \Psi(z) d z
\end{aligned}
$$

The extra term provides $K(0,0)=1$, and since

$$
\mathcal{J}\left(Q, z_{0}\right)=\frac{1}{I_{0}\left(Q z_{0}\right)}
$$

it rapidly decreases with the growth of $Q_{1}$ and/or $Q_{2}$. We will see later that in these regions the behavior of $F_{\pi \gamma^{*} \gamma^{*}}\left(Q_{1}^{2}, Q_{2}^{2}\right)$ is determined by small- $z$ region of integration. Thus, the effects of fixing the $\Psi\left(z_{0}\right) \neq 0$ artifact at the infrared boundary are wiped out in the "short-distance" regime.

\subsection{Momentum Dependence}

\subsubsection{Small Virtualities}

If one of the photons is real $Q_{1}^{2}=0$, while another is almost real, $Q_{2}^{2}=Q^{2} \ll 1 / z_{0}^{2}$, we may use the expansion

$$
\mathcal{J}(Q, z)=1-\frac{1}{4} Q^{2} z^{2}\left[1-\ln \frac{z^{2}}{z_{0}^{2}}\right]+\mathcal{O}\left(Q^{4}\right)
$$

which gives (for $z_{0}=z_{0}^{\rho}$ and $a=a_{0}=2.26$ )

$$
\begin{aligned}
K\left(0, Q^{2}\right) & \simeq 1-0.66 \frac{Q^{2} z_{0}^{2}}{4} \\
& \simeq 1-0.96 \frac{Q^{2}}{m_{\rho}^{2}} .
\end{aligned}
$$

The predicted slope is very close to the value $1 / m_{\rho}^{2}$ expected from a naive vectormeson dominance. Experimentally, the slope of the $F_{\pi \gamma^{*} \gamma^{*}}\left(0, Q^{2}\right)$ form factor for small timelike (negative) $Q^{2}$ is measured through the Dalitz decay $\pi^{0} \rightarrow e^{+} e^{-} \gamma$. In our notations, the usual representation of the results is

$$
K\left(0, Q^{2}\right)=1-a_{\pi} \frac{Q^{2}}{m_{\pi}^{2}},
$$

where $m_{\pi}$ is the experimental pion mass. Then the $Q^{2}$-slope given by Eq. (5.38) corresponds to $a_{\pi} \approx 0.031$. This number is not very far from the (central) values of two recent experiments, $a_{\pi}=0.026 \pm 0.024 \pm 0.0048$ [120], $a_{\pi}=0.025 \pm 0.014 \pm 0.026$ [121], but the experimental errors are rather large. An earlier experiment [122] produced $a_{\pi}=-0.11 \pm 0.03 \pm 0.08$, a result whose (central) value has the opposite 
sign and much larger absolute magnitude. In the spacelike region, data are available only for the values $Q^{2} \gtrsim 0.5 \mathrm{GeV}^{2}$ (CELLO [80]) and $Q^{2} \gtrsim 1.5 \mathrm{GeV}^{2}$ (CLEO [81]) which cannot be treated as very small. The CELLO collaboration [80] gives the value $a_{\pi}=0.0326 \pm 0.0026$ that is very close to our result. To settle the uncertainty of the timelike data (and also on its own grounds), it would be interesting to have data on the slope from the spacelike region of very small $Q^{2}$, which may be obtained by modification of the PRIMEX experiment [123] at JLab.

\subsubsection{Large Virtualities}

Since $\mathcal{J}\left(Q, z_{0}\right)$ exponentially $\sim e^{-Q z_{0}}$ vanishes for large $Q$, we can neglect the first term of Eq. (5.35) in the asymptotic $Q^{2} \rightarrow \infty$ region. Representing $\partial_{z} \psi(z)$ in terms of the $\Phi(z)$ wave function, we write $K\left(Q_{1}^{2}, Q_{2}^{2}\right)$ as

$$
K\left(Q_{1}^{2}, Q_{2}^{2}\right) \simeq \frac{s_{0}}{2} \int_{0}^{z_{0}} \mathcal{J}\left(Q_{1}, z\right) \mathcal{J}\left(Q_{2}, z\right) \Phi(z) z d z .
$$

Our goal is to compare the predictions based on this formula with the leading-order perturbative QCD results. Note that the situation is different from that with the charged pion form factor, where the leading-power $1 / Q^{2}$ pQCD result [77]

$$
F_{\pi}^{\mathrm{pQCD}}\left(Q^{2}\right) \rightarrow 2\left(\alpha_{s} / \pi\right) s_{0} / Q^{2}
$$

corresponding to the hard contribution is the $\mathcal{O}\left(\alpha_{s}\right)$ correction to the soft contribution, for which AdS/QCD gives [38]

$$
F_{\pi}^{\mathrm{AdS} / \mathrm{QCD}}\left(Q^{2}\right) \rightarrow s_{0} / Q^{2}
$$

In that situation, it makes no sense to discuss whether pQCD and AdS/QCD asymptotic predictions agree with each other numerically or not. In general, our AdS/QCD model contains no information about hard gluon exchanges of pQCD that produce the $\alpha_{s}$ factors. However, the pQCD expression for the $\gamma^{*} \gamma^{*} \rightarrow \pi^{0}$ form factor has zero order in $\alpha_{s}$, so now it makes sense to compare the leading-order pQCD predictions for this particular (in fact, exceptional) form factor with AdS/QCD calculations.

It is instructive to consider first two simple kinematic situations $\left(Q_{1}^{2}=0, Q_{2}^{2}=\right.$ $Q^{2}$ and $Q_{1}^{2}=Q_{2}^{2}=Q^{2}$, and then analyze the general case.

\section{One Real Photon}

The form factor in the kinematics where the virtuality of one of the photons can be treated as zero $Q_{1}^{2} \approx 0$, while another $Q_{2}^{2}=Q^{2}$ is large was studied experimentally by the CELLO [80] and CLEO [81] collaborations.

In perturbative $\mathrm{QCD}$, the $K\left(0, Q^{2}\right)$ form factor at large $Q^{2}$ is obtained from the factorization formula

$$
K\left(0, Q^{2}\right)=\int_{0}^{1} T\left(Q^{2}, x\right) \varphi_{\pi}(x) d x
$$


where $T\left(Q^{2}, x\right)$ is the amplitude of the hard subprocess $\gamma \gamma^{*} \rightarrow \bar{q} q$. The latter, modulo logarithms of $Q^{2}$, has a $1 / Q^{2}$ behavior. In the lowest order, when

$$
K^{\mathrm{pQCD}}\left(0, Q^{2}\right)=\frac{s_{0}}{3 Q^{2}} \int_{0}^{1} \frac{\varphi_{\pi}(x)}{x} d x \equiv \frac{s_{0}}{3 Q^{2}} I^{\varphi},
$$

the purely $1 / Q^{2}$ outcome reflects the large- $Q^{2}$ behavior of the hard quark propagator connecting the photon vertices. A particular form of the pion distribution amplitude (DA) is irrelevant to the power of the large- $Q^{2}$ behavior of $K\left(0, Q^{2}\right)$, as far as it provides a convergent $x$-integral in Eq. (5.42). The latter requirement is fulfilled, e.g., if the pion distribution amplitude $\varphi_{\pi}(x)$ vanishes at the end-points $(x=0$ or 1$)$ as a positive power of $x(1-x)$. Whether it vanishes at $x=0$ as $x, x^{2}$ or $\sqrt{x}$ does not matter - this would not affect the $1 / Q^{2}$ large- $Q^{2}$ behavior of the $\gamma \gamma^{*} \pi^{0}$ form factor in the lowest pQCD order. However, the shape of the pion distribution amplitude $\varphi_{\pi}(x)$ determines the value of the coefficient $I^{\varphi}$ that provides the normalization of the $\mathcal{O}\left(1 / Q^{2}\right)$ term. For the asymptotic shape $\varphi_{\pi}^{\text {as }}(x)=6 x(1-x)$, we have $I^{\varphi^{\text {as }}}=3$ and $K^{\mathrm{pQCD}(\text { as) }}\left(0, Q^{2}\right)=s_{0} / Q^{2}$.

Take now our extended AdS/QCD model for the $K\left(0, Q^{2}\right)$ form factor. It gives

$$
K\left(0, Q^{2}\right) \simeq \frac{s_{0}}{2} \int_{0}^{z_{0}} \mathcal{J}(Q, z) \Phi(z) z d z
$$

At first sight, this expression, though completely different analytically from the pQCD formula (5.41), has a general structure similar to it: the $Q^{2}$-dependence is accumulated in the universal current factor $\mathcal{J}(Q, z)$, while the bound state dynamics is described by the $Q^{2}$-independent wave function $\Phi(z)$. The obvious difference is that the bulk-to-boundary propagator $\mathcal{J}(Q, z)$ does not have a power behavior at large $Q^{2}$ : it behaves in that region like $e^{-Q z}$, coinciding in this limit with $z Q K_{1}(z Q) \equiv \mathcal{K}(Q z)$, the free-field version of the nonnormalizable mode. The power behavior in $Q^{2}$ appears only after integration of the exponentially decreasing function over $z$. As a result, only small values of $z$ are important in the relevant integral, and the outcome is determined by the small- $z$ behavior of the wave function $\Phi(z)$. As far as $\Phi(z)$ tends to a nonzero value $\Phi(0)$ when $z \rightarrow 0$, the outcome is the $1 / Q^{2}$ behavior:

$$
K\left(0, Q^{2}\right) \rightarrow \frac{\Phi(0) s_{0}}{2 Q^{2}} \int_{0}^{\infty} d \chi \chi^{2} K_{1}(\chi)=\frac{\Phi(0) s_{0}}{Q^{2}}
$$

Just like in the case of the (charged) pion EM form factor [38], the large- $Q^{2}$ behavior of $K\left(0, Q^{2}\right)$ is determined by the value of the $\Phi(z)$ wave function at the origin. Note, that this value is fixed: $\Phi(0)=1$, which gives $K\left(0, Q^{2}\right)=s_{0} / Q^{2}$, the result that coincides with the leading-order prediction of $\mathrm{pQCD}$ for $I^{\varphi}=3$, the value that is obtained, e.g., if one takes the asymptotic pion DA $\varphi_{\pi}^{\text {as }}(x)=6 x(1-x)$.

Experimentally, the leading-order pQCD prediction with $I^{\varphi}=3$ is somewhat above the data. However, the next-to-leading $\mathcal{O}\left(\alpha_{s}\right)$ pQCD correction is negative, and decreases the result by about $15 \%$, producing a satisfactory agreement. More elaborate fits [88] favor DAs that differ from the asymptotic one by higher Gegenbauer harmonics $x(1-x) C_{n}(2 x-1)$ with $n=2$ and $n=4$. Still, for all the 
DAs obtained from these fits, the magnitude of the integral $I^{\varphi}$ is very close to the value $I^{\text {as }}=3$ given by the asymptotic DA (see Ref. [88] for details and references). Thus, the result of our calculation is in full agreement with the magnitude of the leading-order pQCD part of the NLO fits of existing experimental data.

\section{Equal Virtualities}

Another interesting kinematics is when the photons have equal large virtualities, $Q_{1}^{2}=Q_{2}^{2}=Q^{2}$. In this case, the leading-order pQCD prediction

$$
K^{\mathrm{pQCD}}\left(Q^{2}, Q^{2}\right)=\frac{s_{0}}{3} \int_{0}^{1} \frac{\varphi_{\pi}(x) d x}{x Q^{2}+(1-x) Q^{2}}=\frac{s_{0}}{3 Q^{2}}
$$

does not depend on the shape of the pion DA. In our extended AdS/QCD model, we obtain

$$
K\left(Q^{2}, Q^{2}\right) \simeq \frac{s_{0}}{2} \int_{0}^{z_{0}}[\mathcal{J}(Q, z)]^{2} \Phi(z) z d z
$$

Asymptotically, we have

$$
K\left(Q^{2}, Q^{2}\right) \rightarrow \frac{\Phi(0) s_{0}}{Q^{2}} \int_{0}^{\infty} d \chi \chi^{3}\left[K_{1}(\chi)\right]^{2}=\frac{s_{0}}{3 Q^{2}}
$$

which is the same result as in the leading-order pQCD.

\section{General Case}

Finally, let us consider the general kinematics, when $Q_{1}^{2}=(1+\omega) Q^{2}$, and $Q_{2}^{2}=$ $(1-\omega) Q^{2}$. The leading-order pQCD formula gives in this case

$$
K^{\mathrm{pQCD}}\left((1+\omega) Q^{2},(1-\omega) Q^{2}\right)=\frac{s_{0}}{3 Q^{2}} \int_{0}^{1} \frac{\varphi_{\pi}(x) d x}{1+\omega(2 x-1)} \equiv \frac{s_{0}}{3 Q^{2}} I^{\varphi}(\omega),
$$

while Eq. (5.40) of our AdS/QCD model reduces, for large $Q^{2}$, to

$$
\begin{aligned}
K\left((1+\omega) Q^{2},(1-\omega) Q^{2}\right) & \rightarrow \frac{\Phi(0) s_{0}}{2 Q^{2}} \sqrt{1-\omega^{2}} \int_{0}^{\infty} d \chi \chi^{3} K_{1}(\chi \sqrt{1+\omega}) K_{1}(\chi \sqrt{1-\omega}) \\
& \equiv \frac{s_{0}}{3 Q^{2}} I^{\operatorname{AdS}}(\omega)
\end{aligned}
$$

with the function $I^{\operatorname{AdS}}(\omega)$ given by

$$
I^{\operatorname{AdS}}(\omega)=\frac{3}{4 \omega^{3}}\left[2 \omega-\left(1-\omega^{2}\right) \ln \left(\frac{1-\omega}{1+\omega}\right)\right] .
$$

It is straightforward to check that $I^{\mathrm{AdS}}(\omega)$ coincides with the pQCD function $I^{\varphi}(\omega)$ calculated for the asymptotic distribution amplitude $\varphi^{\text {as }}(x)=6 x(1-x)$. Indeed, using the representation

$$
\chi K_{1}(\chi)=\int_{0}^{\infty} e^{-\chi^{2} / 4 u-u} d u
$$


we can easily integrate over $\chi$ in Eq. (5.49) to get

$$
K\left(Q_{1}^{2}, Q_{2}^{2}\right) \rightarrow \frac{s_{0}}{Q^{2}} \int_{0}^{\infty} \int_{0}^{\infty} \frac{u_{1} u_{2} e^{-u_{1}-u_{2}} d u_{1} d u_{2}}{u_{2}(1+\omega)+u_{1}(1-\omega)}
$$

Changing variables $u_{2}=x \lambda, u_{1}=(1-x) \lambda$ and integrating over $\lambda$, we obtain

$$
K\left(Q_{1}^{2}, Q_{2}^{2}\right) \rightarrow \frac{s_{0}}{3 Q^{2}} \int_{0}^{1} \frac{6 x(1-x) d x}{1+\omega(2 x-1)},
$$

which coincides with the pQCD formula (5.45) if we take $\varphi_{\pi}(x)=6 x(1-x)$.

Note that the absolute normalization of the asymptotic behavior of $K\left(Q_{1}^{2}, Q_{2}^{2}\right)$ for large $Q_{i}$ in our model is fixed by the choice $k=2$ that allows to conform to the value $K\left(Q_{1}^{2}, Q_{2}^{2}\right)=1$ corresponding to the QCD anomaly result. As we have seen, this choice exactly reproduces also the leading-order pQCD result for the equal-virtualities form factor $K\left(Q^{2}, Q^{2}\right)$. The origin of this rather unexpected result needs further studies. Recall also that in pQCD the result for $K\left(Q^{2}, Q^{2}\right)$ is the same for any pion distribution amplitude, while the result for the unequalvirtualities form factor $K\left((1+\omega) Q^{2},(1-\omega) Q^{2}\right)$ depends on the choice of the pion distribution amplitude. The fact that our AdS/QCD model gives the same result as the leading-order pQCD calculation performed for the asymptotic distribution amplitude, also deserves a further investigation.

\section{From Small to Large $Q^{2}$}

Both $K\left(0, Q^{2}\right)$ and $K\left(Q^{2}, Q^{2}\right)$ functions are equal to 1 at $Q^{2}=0$. For large $Q^{2}$, the first one tends to $s_{0} / Q^{2}$ and the second one to $s_{0} / 3 Q^{2}$. The question is how these functions interpolate between the regions of small and large $Q^{2}$. Long ago, Brodsky and Lepage [74] proposed a simple monopole (BL) interpolation

$$
K^{\mathrm{BL}}\left(0, Q^{2}\right)=\frac{1}{1+Q^{2} / s_{0}}
$$

between the $Q^{2}=0$ value and the large- $Q^{2}$ asymptotic prediction of perturbative QCD for $K\left(0, Q^{2}\right)$. Later, this behavior was obtained within the "local quark-hadron duality" approach $[124,56]$, in which $K\left(0, Q^{2}\right)$ is obtained by integrating the spectral density $\rho\left(s, 0, Q^{2}\right)=Q^{2} /\left(s+Q^{2}\right)^{2}$ of the 3-point function over the "pion duality interval" $0 \leqslant s \leqslant s_{0}$. The curve for $Q^{2} K\left(0, Q^{2}\right)$ based on Eq. (5.43) practically coincides with that based on BL interpolation (5.54), see Figs. 5.2 and 5.3. For comparison, we also show on Fig. 5.2 the monopole fit $K^{\mathrm{CLEO}}\left(0, Q^{2}\right)=1 /\left(1+Q^{2} / \Lambda_{\pi}^{2}\right)$ (with $\Lambda_{\pi}=776 \mathrm{MeV}$ ) of CLEO data [81]. As we mentioned, an accurate fit to CLEO data [88] was obtained in the next-to-leading order (NLO) pQCD, with the leading order part of the NLO pQCD fit being very close to BL-interpolation curve, and hence, to our AdS/QCD result as well.

In the case of $K\left(Q^{2}, Q^{2}\right)$ function, our model predicts the slope $1.92 / m_{\rho}^{2} \approx$ $2.15 / s_{0}$ (twice the slope of $K\left(0, Q^{2}\right)$, see Eq. (5.38)), while the local duality model 


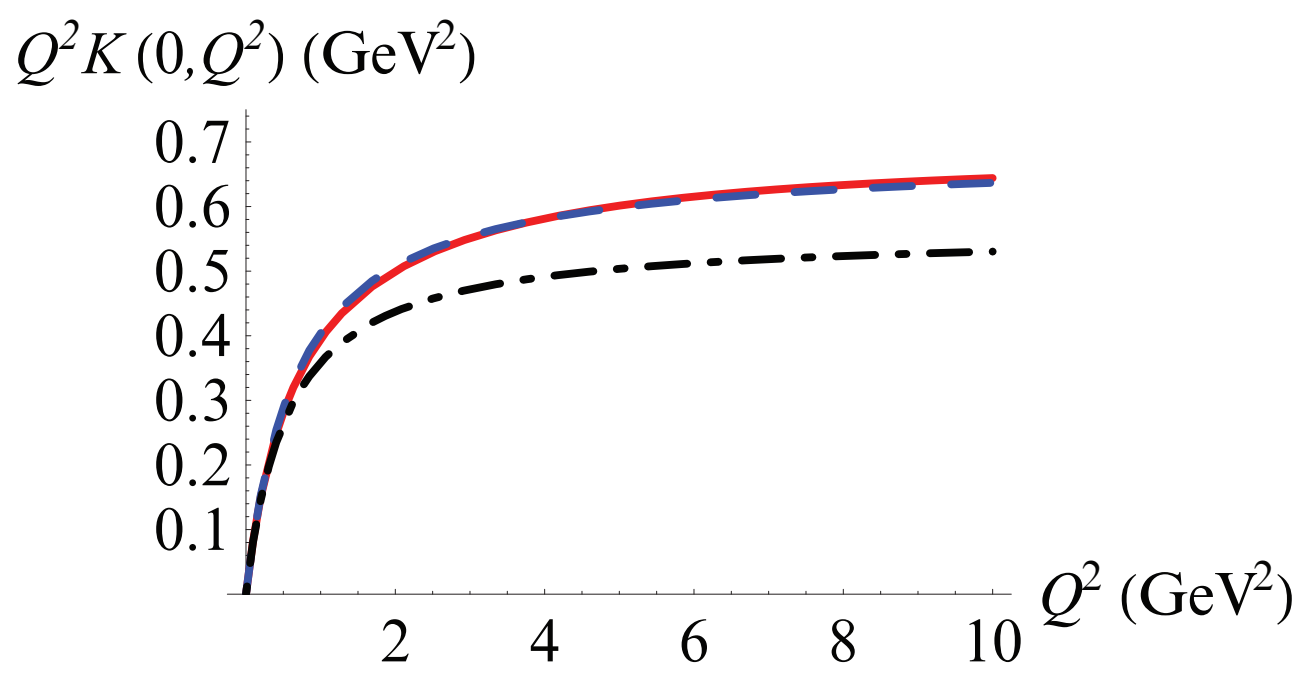

Figure 5.2: Function $Q^{2} K\left(0, Q^{2}\right)$ in AdS/QCD model (solid curve, red online) and in local quark hadron duality model (coinciding with Brodsky-Lepage interpolation formula, dashed curve, blue online). The monopole fit of CLEO data is shown by dash-dotted curve (black online).

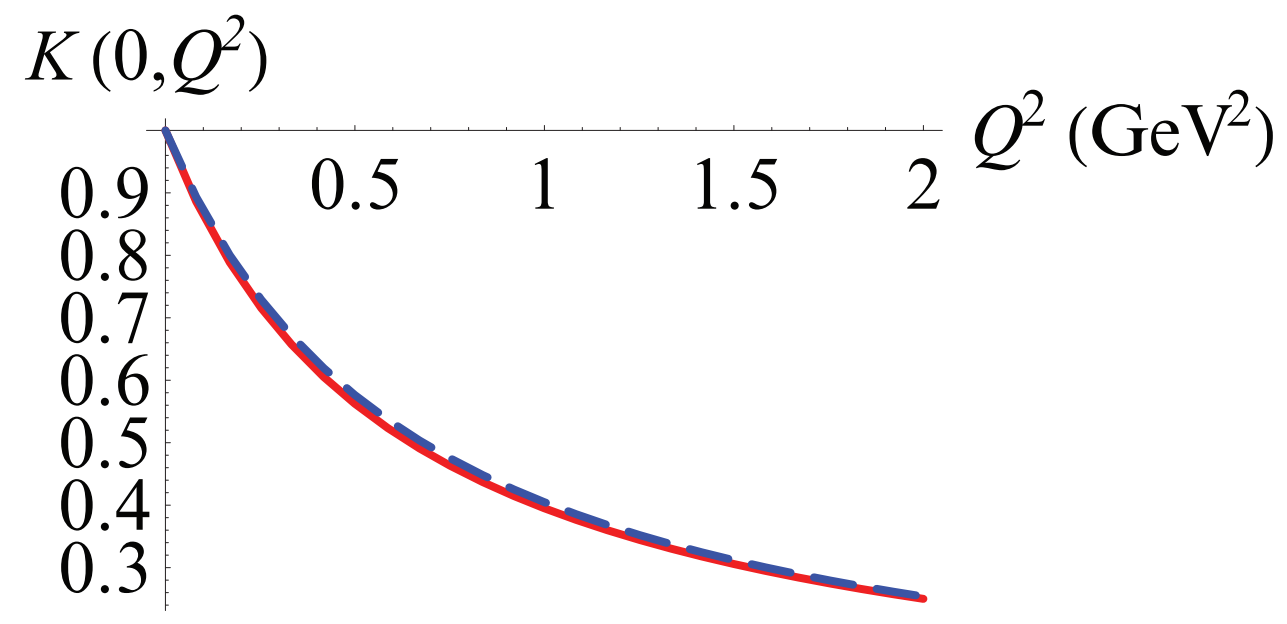

Figure 5.3: Form factor $K\left(0, Q^{2}\right)$ in AdS/QCD model (solid curve, red online) compared to BL interpolation formula (dashed curve, blue online). 


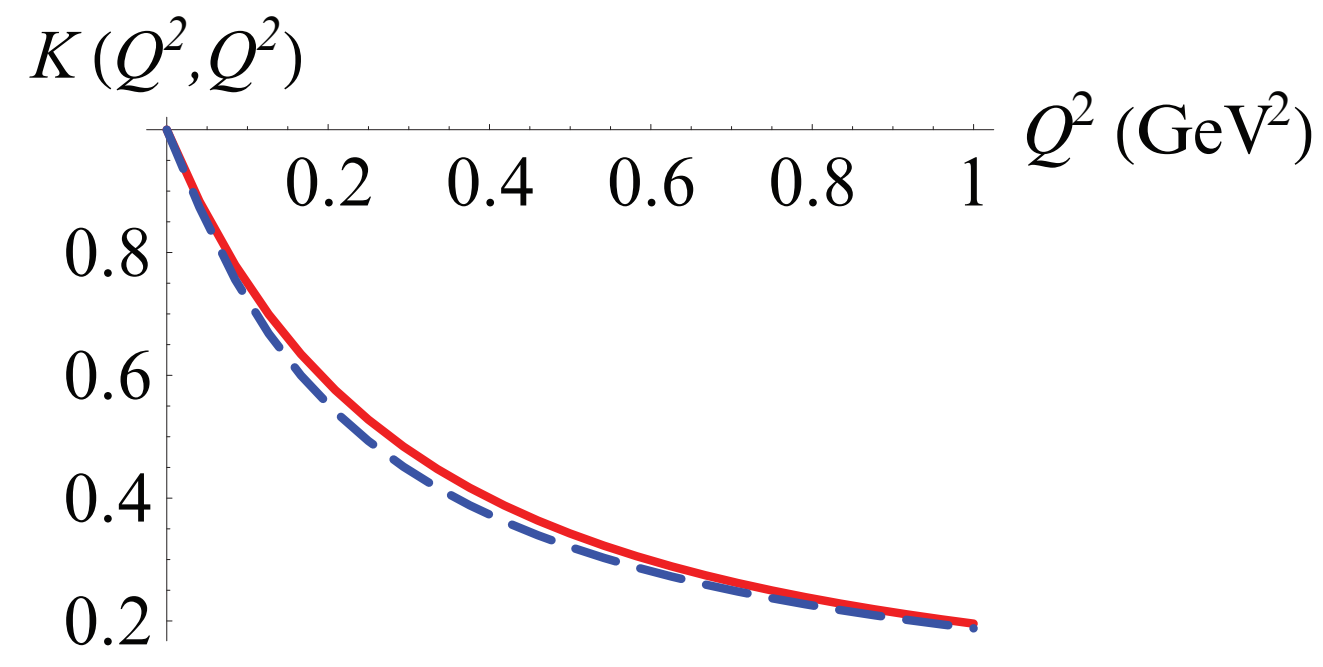

Figure 5.4: Form factor $K\left(Q^{2}, Q^{2}\right)$ in AdS/QCD model (solid curve, red online) compared to the local quark-hadron duality model prediction (dashed curve, blue online).

gives [56]

$$
\begin{aligned}
& K^{\mathrm{LD}}\left(Q^{2}, Q^{2}\right)=1-Q^{4} \int_{0}^{1} \frac{d x}{\left[Q^{2}+s_{0} x(1-x)\right]^{2}} \\
= & 1-\frac{2 Q^{2}}{s_{0}+4 Q^{2}}-\frac{8 Q^{4} \tanh ^{-1} \sqrt{s_{0} /\left(s_{0}+4 Q^{2}\right)}}{\sqrt{s_{0}\left(s_{0}+4 Q^{2}\right)^{3}}},
\end{aligned}
$$

a curve which has the slope $2 / s_{0}$ at $Q^{2}=0$ :

$$
K^{\mathrm{LD}}\left(Q^{2}, Q^{2}\right)=1-2 \frac{Q^{2}}{s_{0}}+\mathcal{O}\left(Q^{4}\right) .
$$

However, higher terms of $Q^{2}$ expansion become important for $Q^{2}$ as small as 0.01 $\mathrm{GeV}^{2}$, where $K^{\operatorname{AdS}}\left(Q^{2}, Q^{2}\right)$ becomes larger than $K^{\mathrm{LD}}\left(Q^{2}, Q^{2}\right)$, and the ratio

$$
K^{\mathrm{AdS}}\left(Q^{2}, Q^{2}\right) / K^{\mathrm{LD}}\left(Q^{2}, Q^{2}\right)
$$

reaches its maximum value of 1.08 for $Q^{2} \sim 0.3 \mathrm{GeV}^{2}$, then slowly decreasing towards the limiting value of 1 . For large $Q^{2}$, the local duality model gives the same result

$$
K^{\mathrm{LD}}\left(Q^{2}, Q^{2}\right)=\frac{s_{0}}{3 Q^{2}}+\mathcal{O}\left(1 / Q^{4}\right)
$$

as our present model (5.47) and pQCD (5.45). As a consequence, our present model produces a curve that is very close to the curve based on the local duality model, see Fig. 5.4. 


\subsection{Bound-State Decomposition}

The bulk-to-boundary propagator $\mathcal{J}(Q, z)$ may be written as a sum

$$
\mathcal{J}(Q, z)=\sum_{n=1}^{\infty} \frac{g_{5} f_{n} \psi_{n}^{V}(z)}{Q^{2}+M_{n}^{2}}
$$

over all vector bound states. For this reason, the form factor $K\left(Q_{1}^{2}, Q_{2}^{2}\right)$ also has a generalized vector meson dominance (GVMD) representation

$$
K\left(Q_{1}^{2}, Q_{2}^{2}\right)=\sum_{n=1}^{\infty} \sum_{k=1}^{\infty} \frac{A_{n, k}+B_{n, k}}{\left(1+Q_{1}^{2} / M_{n}^{2}\right)\left(1+Q_{2}^{2} / M_{k}^{2}\right)},
$$

where $A_{n, k}$ 's come from the first (surface) term in Eq.(5.35),

$$
A_{n, k}=\frac{4 \Psi\left(z_{0}\right)}{\gamma_{0, n} \gamma_{0, k} J_{1}\left(\gamma_{0, n}\right) J_{1}\left(\gamma_{0, k}\right)}
$$

while $B_{n, k}$ 's are obtained from the second term and are given by the convolutions

$$
B_{n, k}=-\frac{4}{z_{0}^{2} \gamma_{0, n} \gamma_{0, k} J_{1}^{2}\left(\gamma_{0, n}\right) J_{1}^{2}\left(\gamma_{0, k}\right)} \int_{0}^{z_{0}} \Psi^{\prime}(z) J_{1}\left(\gamma_{0, n} z / z_{0}\right) J_{1}\left(\gamma_{0, k} z / z_{0}\right) z^{2} d z
$$

Let us study the structure of the bound-state decomposition in two most interesting cases: for $K\left(0, Q^{2}\right)$ and $K\left(Q^{2}, Q^{2}\right)$.

\subsubsection{One Real Photon}

To study the bound-state decomposition of $K\left(0, Q^{2}\right)$, we write the basic expression

$$
K\left(0, Q^{2}\right)=\Psi\left(z_{0}\right) \mathcal{J}\left(Q, z_{0}\right)-\int_{0}^{z_{0}} \mathcal{J}(Q, z) \Psi^{\prime}(z) d z
$$

and use GVMD representation (5.58) for $\mathcal{J}(Q, z)$. This gives

$$
K\left(0, Q^{2}\right)=\sum_{n=1}^{\infty} \frac{A_{n}+B_{n}}{1+Q^{2} / M_{n}^{2}}
$$

where

$$
A_{n}=\frac{2 \Psi\left(z_{0}\right)}{\gamma_{0, n} J_{1}\left(\gamma_{0, n}\right)}
$$

and $B_{n}$ 's coincide with the coefficients

$$
B_{n}=-\frac{2}{z_{0} \gamma_{0, n} J_{1}^{2}\left(\gamma_{0, n}\right)} \int_{0}^{z_{0}} \Psi^{\prime}(z) J_{1}\left(\gamma_{0, n} z / z_{0}\right) z d z
$$


for the expansion

$$
\Psi^{\prime}(z)=-\frac{1}{z_{0}} \sum_{n=1}^{\infty} B_{n} \gamma_{0, n} J_{1}\left(\gamma_{0, n} z / z_{0}\right)
$$

of the pion wave function $\Psi^{\prime}(z)$ over the $\psi_{n}^{V}(z) / z$ wave functions (5.7) of vector meson bound states. In particular,

$$
\begin{aligned}
K(0,0) & =\sum_{n=1}^{\infty} A_{n}+\sum_{n=1}^{\infty} B_{n} \\
& =\Psi\left(z_{0}\right)+\left[\Psi(0)-\Psi\left(z_{0}\right)\right]=1 .
\end{aligned}
$$

This relation may be directly obtained from the formula

$$
\left.2 \sum_{n=1}^{\infty} \frac{x J_{1}\left(\gamma_{0, n} x\right)}{\gamma_{0, n} J_{1}^{2}\left(\gamma_{0, n}\right)}\right|_{x \leqslant 1}=1
$$

The bound state decomposition of $K(0,0)$ looks like

$$
\begin{aligned}
K(0,0)=1=0.9512 & +0.0408+0.0446-0.0753 \\
& +0.0764-0.0736+0.0703+\ldots .
\end{aligned}
$$

There is a strong dominance of the lowest vector state, while each of the higher states is suppressed by more than factor of 10 . The slow convergence of higher terms is due to $A_{n}$ terms proportional to $\Psi\left(z_{0}\right) \approx 0.14$. For large $n$, one can approximate $A_{n} \approx \Psi\left(z_{0}\right)(-1)^{n} \sqrt{2 / n}$

Integrating by parts in Eq. (5.65) gives a representation directly for the total coefficient

$$
A_{n}+B_{n}=\frac{2}{z_{0}^{2} J_{1}^{2}\left(\gamma_{0, n}\right)} \int_{0}^{z_{0}} \Psi(z) J_{0}\left(\gamma_{0, n} z / z_{0}\right) z d z
$$

that is related to the expansion of the pion wave function $\Psi(z)$ over the $\phi_{n}$-functions (5.7) of vector meson bound states:

$$
\Psi(z)=\sum_{n=1}^{\infty}\left(A_{n}+B_{n}\right) J_{0}\left(\gamma_{0, n} z / z_{0}\right) .
$$

Using it, one obtains again that $K(0,0)=\Psi(0)=1$. The slow convergence of higher terms in Eq. (5.69) is now explained by the necessity to reproduce the finite value of $\Psi(z)$ at $z=z_{0}$ by functions vanishing at $z=z_{0}$.

The slope of $K\left(0, Q^{2}\right)$ at $Q^{2}=0$ is given by the sum of $\left(A_{n}+B_{n}\right) / M_{n}^{2}$ coefficients, which converges rather fast:

$$
\begin{aligned}
K\left(0, Q^{2}\right)=1- & \frac{Q^{2}}{m_{\rho}^{2}}\{0.9512+0.0077+0.0034-0.0031 \\
& +0.0020-0.0013+0.0009+\ldots\}
\end{aligned}
$$


and the contribution of the lowest state completely dominates the outcome.

Each term of the GVMD expansion (5.63) behaves like $1 / Q^{2}$ at large $Q^{2}$. In particular, the lowest-state contribution behaves like

$$
0.95 m_{\rho}^{2} / Q^{2} \approx\left(0.57 \mathrm{GeV}^{2}\right) / Q^{2}
$$

We also obtained above that $K\left(0, Q^{2}\right)$ behaves asymptotically like

$$
s_{0} / Q^{2} \approx\left(0.67 \mathrm{GeV}^{2}\right) / Q^{2}
$$

The two scales are not so different, and one may be tempted to speculate that the large- $Q^{2}$ behavior of $K\left(0, Q^{2}\right)$ also reflects the dominance of the lowest resonance. However, the coefficient of $1 / Q^{2}$ is formally given by the sum of $\left(A_{n}+B_{n}\right) M_{n}^{2}$ terms, which does not show good convergence even after 7 terms are taken:

$$
\begin{gathered}
\sum_{n=1}^{\infty}\left(A_{n}+B_{n}\right) M_{n}^{2}=m_{\rho}^{2}\{0.951+0.215+0.577 \\
\quad-1.811+2.945-4.158+5.473+\ldots\} .
\end{gathered}
$$

A simple comment is in order now: within the AdS/QCD model [26] the $\rho$-meson mass is determined by the "confinement radius" $z_{0}$, while the scale $s_{0}=8 \pi^{2} f_{\pi}^{2}$ is basically determined by the chiral symmetry breaking parameter $\alpha$ (see Eq. (5.15) and preceding discussion). Calculationally, the coefficient $s_{0}$ of $1 / Q^{2}$ asymptotic behavior was determined solely by the magnitude of the pion wave function $\Psi^{\prime}(z) / z$ at the origin. Furthermore, it was legitimate to take the free-field form of the vector bulk-to-boundary propagator in our calculation, i.e., no information about vector channel mass scales was involved.

Moreover, one may write the bound-state decomposition for the $J\left(Q, z_{0}\right)$ function. Again, each term of such a decomposition has $1 / Q^{2}$ asymptotic behavior, while $J\left(Q, z_{0}\right)$ exponentially decreases for large $Q$. In fact, the formal sum $\sum_{n} A_{n} M_{n}^{2}$ in this case diverges like $\sum_{n}(-1)^{n} n^{3 / 2}$.

Summarizing, the $1 / Q^{2}$ asymptotic behavior of $K\left(0, Q^{2}\right)$ has nothing to do with the fact that the contribution of each particular bound state has $1 / Q^{2}$ behavior. If, instead of $\Phi(z)$, one would take a function with $\sim z^{\Delta}$ behavior for small $z$, one would still be able to write the GVMD representation for such a version of $K\left(0, Q^{2}\right)$, but its asymptotic behavior will be $\sim 1 / Q^{2+\Delta}$.

\subsubsection{Two Deeply Virtual Photons}

Each term of the bound-state decomposition (5.59) for $K\left(Q^{2}, Q^{2}\right)$ has $1 / Q^{4}$ behavior. Thus, in the case of strong dominance of a few lowest states, one would expect $1 / Q^{4}$ large- $Q^{2}$ behavior of this function.

However, as we already obtained, the function $K\left(Q^{2}, Q^{2}\right)$ behaves like $1 / Q^{2}$ for large $Q^{2}$. This result was a consequence of two features of the form factor integral (5.40). The first is the fact that the bulk-to-boundary propagator $\mathcal{J}(Q, z)$ behaves 
like $e^{-Q z}$ for large $Q$. This is a very general property: in this limit $\mathcal{J}(Q, z)$ should coincide with its free-field version $\mathcal{K}(Q z)=z Q K_{1}(z Q)$. The second feature is that the pion wave function $\Phi(z)$ is finite at the origin, which follows from the basic formula (5.11) that defines $f_{\pi}$.

Hence, to qualitatively understand the mechanism that produces a $1 / Q^{2}$ result from the doubly-infinite sum of $1 / Q^{4}$ terms, one may consider a simpler "toy" model that also has these general properties, but allows one to analytically calculate integrals that determine the coefficients in Eq. (5.59). In particular, an explicit result for $K\left(Q_{1}^{2}, Q_{2}^{2}\right)$ may be obtained if we take the soft-model expression [37]

$$
\mathcal{J}^{\mathrm{s}}(Q, z)=a \int_{0}^{1} x^{a-1} \exp \left[-\frac{x}{1-x} \kappa^{2} z^{2}\right] d x
$$

for the bulk-to-boundary propagators (with $\kappa$ being the oscillator parameter and $\left.a=Q^{2} / 4 \kappa^{2}\right)$ and

$$
\Psi^{\mathrm{S}}(z)=e^{-\kappa^{2} z^{2}}
$$

for the pion wave function. This model has the required properties, namely, $\mathcal{J}^{\mathrm{s}}(Q, z)$ approaches the free-field function $\mathcal{K}(Q z)$ for large $Q^{2}$, while $\Phi(0)$ is finite.

Calculating the integral

$$
K^{\mathrm{s}}\left(Q_{1}^{2}, Q_{2}^{2}\right)=2 \kappa^{2} \int_{0}^{\infty} \mathcal{J}^{\mathrm{s}}\left(Q_{1}, z\right) \mathcal{J}^{\mathrm{s}}\left(Q_{2}, z\right) e^{-\kappa^{2} z^{2}} z d z
$$

gives

$$
K^{\mathrm{s}}\left(Q_{1}^{2}, Q_{2}^{2}\right)=\sum_{n=0}^{\infty} \frac{a_{1}}{\left(a_{1}+n\right)\left(a_{1}+n+1\right)} \frac{a_{2}}{\left(a_{2}+n\right)\left(a_{2}+n+1\right)}
$$

where $a_{1}=Q_{1}^{2} / M^{2}, a_{2}=Q_{2}^{2} / M^{2}$ and $M=2 \kappa$ is the mass of the lowest bound state. The spectrum corresponding to $\mathcal{J}^{\mathrm{s}}(Q, z)$ is given by $M_{m}^{2}=m M^{2}$, with $m=1,2, \ldots$, and Eq. (5.77) explicitly displays the bound state poles. For large $Q_{1}^{2}, Q_{2}^{2}$, each term of this sum behaves like $1 / Q_{1}^{2} Q_{2}^{2}$. However, taking $a_{1}=a_{2}=a \gg 1$ gives

$$
K^{\mathrm{s}}\left(Q^{2}, Q^{2}\right) \rightarrow a^{2} \int_{0}^{\infty} \frac{d n}{(n+a)^{4}}=\frac{1}{3 a}=\frac{M^{2}}{3 Q^{2}} .
$$

Thus, the conversion from the $1 / Q^{4}$ asymptotics of individual terms to the $1 / Q^{2}$ asymptotics of the sum is due to nondecreasing $\mathcal{O}\left(n^{0}\right)$ behavior of the coefficients accompanying $n^{\text {th }}$ term of the sum. In other words, transitions involving higher bound states are important, i.e., the pole decomposition is far from being dominated by a few lowest states. 


\subsubsection{Structure of Two-Channel Pole Decomposition}

However, to make specific statements about the transitions, one should realize that Eq. (5.77) does not have the form of Eq. (5.59). In particular, it is not a double sum, and having a sum over a single parameter implies that the summation parameters $n$ and $k$ in the double sum representation are correlated. A simple inspection of Eq. (5.77) shows that either $k=n$ or $k=n \pm 1$. Furthermore, the representation (5.77) has $Q_{1}^{2} Q_{2}^{2}$ factor in the numerator, which should be canceled against the denominator factors to get an expression in which $Q_{1}^{2}$ and $Q_{2}^{2}$ appear in denominators only. The easiest way to obtain the desired GVMD-type expansion for $K^{\mathrm{s}}\left(Q_{1}^{2}, Q_{2}^{2}\right)$ is to use another representation [37]

$$
\mathcal{J}^{\mathrm{s}}(Q, z)=\kappa^{2} z^{2} \int_{0}^{1} \exp \left[-\frac{x}{1-x} \kappa^{2} z^{2}\right] \frac{x^{a} d x}{(1-x)^{2}}
$$

for the bulk-to-boundary propagators. Then

$$
K^{\mathrm{s}}\left(Q_{1}^{2}, Q_{2}^{2}\right)=\sum_{n=0}^{\infty} \frac{(n+1)(n+2)}{\left(a_{1}+n+1\right)\left(a_{1}+n+2\right)\left(a_{2}+n+1\right)\left(a_{2}+n+2\right)} .
$$

Now, each term of the sum decreases as $1 / Q_{1}^{4} Q_{2}^{4}$, but the sum may be rewritten as

$$
K^{\mathrm{s}}\left(Q_{1}^{2}, Q_{2}^{2}\right)=\sum_{n=1}^{\infty} \frac{1}{1+Q_{1}^{2} / M_{n}^{2}}\left\{\frac{2}{1+Q_{2}^{2} / M_{n}^{2}}-\frac{1}{1+Q_{2}^{2} / M_{n+1}^{2}}-\frac{1}{1+Q_{2}^{2} / M_{n-1}^{2}}\right\}
$$

i.e., the coefficients $B_{n, k}^{\mathrm{s}}$ of the bound-state expansion (5.59) in this model are given by

$$
B_{n, k}^{\mathrm{s}}=2 \delta_{n, k}-\delta_{n, k+1}-\delta_{n, k-1}
$$

(there is no need to add surface terms in this model since $\Psi^{\mathrm{s}}(\infty)=0$, and hence $\left.A_{n, k}^{\mathrm{s}}=0\right)$.

When $Q_{2}^{2}=0$, only the $n=1$ term contributes, and $K\left(Q^{2}, 0\right)$ in this model is formally dominated by the lowest resonance:

$$
K^{\mathrm{s}}\left(Q^{2}, 0\right)=\frac{1}{1+Q^{2} / M_{1}^{2}}
$$

This fact may create an impression that the $\pi^{0} \rightarrow \gamma \gamma$ decay in this model is dominated by the $\rho \omega$ intermediate state. However, the outcome that the sums of bracketed terms are zero for $n \geq 2$ is a result of cancellation of the contribution of a diagonal transition that gives 2 , and two off-diagonal transitions, each of which gives -1 .

In fact, the coefficients $B_{n, n}^{\mathrm{s}}=2$ of diagonal transitions do not depend on $n$, and their total contribution into $K^{\mathrm{s}}\left(Q_{1}^{2}, Q_{2}^{2}\right)$ diverges. On the other hand, the coefficients 
Table 5.1: Coefficients $B_{n, k}$ in the hard-wall model.

\begin{tabular}{|c|c|c|c|c|c|}
\hline$k \backslash n$ & 1 & 2 & 3 & 4 & 5 \\
\hline 1 & 0.7905 & 0.0272 & -0.1331 & 0.0562 & -0.0275 \\
\hline 2 & 0.0272 & 0.2484 & -0.0423 & -0.0608 & 0.0287 \\
\hline 3 & -0.1331 & -0.0423 & 0.1624 & -0.0367 & -0.0403 \\
\hline 4 & 0.0562 & -0.0608 & -0.0367 & 0.1199 & -0.0303 \\
\hline 5 & -0.0275 & 0.0287 & -0.0403 & -0.0303 & 0.0951 \\
\hline
\end{tabular}

Table 5.2: Coefficients $A_{n, k}$ in the hard-wall model.

\begin{tabular}{|c|c|c|c|c|c|}
\hline$k \backslash n$ & 1 & 2 & 3 & 4 & 5 \\
\hline 1 & 0.3641 & -0.2420 & 0.1935 & -0.1658 & 0.1474 \\
\hline 2 & -0.2420 & 0.1609 & -0.1286 & 0.1102 & -0.0980 \\
\hline 3 & 0.1935 & -0.1286 & 0.1028 & -0.0881 & 0.0783 \\
\hline 4 & -0.1658 & 0.1102 & -0.0881 & 0.0755 & -0.0671 \\
\hline 5 & 0.1474 & -0.0980 & 0.0783 & -0.0671 & 0.0597 \\
\hline
\end{tabular}

$B_{n, n+1}^{\mathrm{s}}=-1$ and $B_{n+1, n}^{\mathrm{s}}=-1$ of subdiagonal transitions are negative, and also do not depend on $n$. The total contribution into $K^{\mathrm{s}}\left(Q_{1}^{2}, Q_{2}^{2}\right)$ of each of $k=n+1$ or $k=n-1$ off-diagonal transitions also diverges. In such a situation, claiming the dominance of the lowest states contribution makes no sense.

In the hard-wall model, the diagonal coefficients $B_{n, n}$ decrease with $n$, and they are visibly larger than the neighboring off-diagonal ones (see Table I). Thus, one may say that, for small $Q_{1}^{2}, Q_{2}^{2}$, the value of $K\left(Q_{1}^{2}, Q_{2}^{2}\right)$ is dominated by the lowest bound states (the coefficients $A_{n, n}$ also decrease with $n$, see Table II, asymptotically they behave like $2 \Psi\left(z_{0}\right) / n$ ).

However, the large- $Q_{1}^{2}, Q_{2}^{2}$ expansion is given by

$$
K\left(Q_{1}^{2}, Q_{2}^{2}\right)=\frac{M_{1}^{4}}{Q_{1}^{2} Q_{2}^{2}} \sum_{n, k=1}^{\infty} \frac{M_{n}^{2} M_{k}^{2}}{M_{1}^{4}}\left(A_{n, k}+B_{n, k}\right)+\ldots,
$$

i.e., one should deal with the coefficients

$$
B_{n, k} \frac{M_{n}^{2} M_{k}^{2}}{M_{1}^{4}}=B_{n, k} \frac{\gamma_{0, n}^{2} \gamma_{0, k}^{2}}{\gamma_{0,1}^{4}} \text { and } A_{n, k} \frac{M_{n}^{2} M_{k}^{2}}{M_{1}^{4}}
$$

the lowest of which are given in Tables III and IV. Again, the coefficients increase with $n$ and $k$ producing divergent series, just like in the toy soft-like model. Note, that asymptotically the sum of $A$-type terms gives a contribution exponentially decreasing with $Q_{1}$ and/or $Q_{2}$, i.e., much faster than the $\sim 1 / Q^{4}$ asymptotic behavior of each particular transition. On the other hand, the sum of $B$-type terms gives a contribution that has $\sim 1 / Q^{2}$ asymptotic behavior, i.e., it drops slower than $1 / Q^{4}$. 
Table 5.3: Coefficients $B_{n, k} M_{n}^{2} M_{k}^{2} / M_{1}^{4}$ in the hard-wall model.

\begin{tabular}{|c|c|c|c|c|c|}
\hline$k \backslash n$ & 1 & 2 & 3 & 4 & 5 \\
\hline 1 & 0.7905 & 0.1433 & -1.7236 & 1.3514 & -1.0593 \\
\hline 2 & 0.1433 & 6.8952 & -2.8875 & -7.6956 & 5.8234 \\
\hline 3 & -1.7236 & -2.8875 & 27.2323 & -11.4306 & -20.1136 \\
\hline 4 & 1.3514 & -7.6956 & -11.4306 & 69.3299 & -28.0684 \\
\hline 5 & -1.0593 & 5.8234 & -20.1136 & -28.0684 & 141.2499 \\
\hline
\end{tabular}

Table 5.4: Coefficients $A_{n, k} M_{n}^{2} M_{k}^{2} / M_{1}^{4}$ in the hard-wall model.

\begin{tabular}{|c|c|c|c|c|c|}
\hline$k \backslash n$ & 1 & 2 & 3 & 4 & 5 \\
\hline 1 & 0.3641 & -1.2751 & 2.5056 & -3.9868 & 5.6816 \\
\hline 2 & -1.2751 & 4.4655 & -8.775 & 13.9624 & -19.8979 \\
\hline 3 & 2.5056 & -8.775 & 17.2437 & -27.4374 & 39.1011 \\
\hline 4 & -3.9868 & 13.9624 & -27.4374 & 43.6572 & -62.216 \\
\hline 5 & 5.6816 & -19.8979 & 39.1011 & -62.216 & 88.6642 \\
\hline
\end{tabular}

The convergence situation may be different in the real-world QCD, in which higher resonances are broad, with the width increasing with $n$ (or $k$ ). Then the diagonal and neighboring non-diagonal transitions strongly overlap for large $n$ and may essentially cancel each other.

\subsection{Summary}

At the end of the pioneering paper [26], it was indicated that one of the future developments of the holographic models would be an incorporation of the 5D ChernSimons term to reproduce the chiral anomaly of QCD. However, only relatively recently Ref. [114] discussed a holographic model of QCD that includes ChernSimons term (see also [125]) and, furthermore, extends the gauged $S U(2)_{L} \otimes S U(2)_{R}$ flavor group to $U(2)_{L} \otimes U(2)_{R}$.

In the present chapter, we develop an extension of the AdS/QCD model, similar in form to that proposed in [114], but adjusted to study the anomalous coupling of the neutral pion to two (in general, virtual) photons. The additional part of the gauge field in the 5D bulk is associated with the isoscalar vector current (related to $\omega$-like mesons). The Chern-Simons term allows to reproduce the tensor structure of the anomalous form factor $F_{\gamma^{*} \gamma^{*} \pi^{0}}\left(Q_{1}^{2}, Q_{2}^{2}\right)$. To exactly reproduce the QCD anomaly result for real photons, we added contributions localized at the IR boundary $z=z_{0}$, and then studied the momentum dependence of the $F_{\gamma^{*} \gamma^{*} \pi^{0}}\left(Q_{1}^{2}, Q_{2}^{2}\right)$ form factor in our model.

In particular, we calculated the slope of the form factor with one real and one 
slightly off-shell photon. Our result $a_{\pi} \approx 0.031$ for the parameter of the usual

$$
F_{\gamma^{*} \gamma^{*} \pi^{0}}\left(0, Q^{2}\right)=F_{\gamma^{*} \gamma^{*} \pi^{0}}(0,0)\left(1-a_{\pi} Q^{2} / m_{\pi}^{2}\right)
$$

low- $Q^{2}$ experimental representation of the data is very close to the value $a_{\pi}=$ $0.0326 \pm 0.0026$ obtained by CELLO collaboration [80] from spacelike $Q^{2}$ measurements, and rather close to the central values $a_{\pi} \sim 0.024$ of two most recent experiments [120, 121] for timelike $Q^{2}$.

Although the holographic model is expected to work for low energies, where QCD is in the strong coupling regime, we found it interesting to investigate the behavior of the model form factor also in the regions where at least one of the photon virtualities is large. For the case with one real and one highly virtual photon, we demonstrated that our AdS/QCD result is in full agreement with the magnitude of the leadingorder part of the next-to-leading-order pQCD fits of existing experimental data. In the kinematics where both photons have equal and large virtualities we obtained the same result as in the leading-order pQCD. Finally, we considered the general case of unequal and large photon virtualities. In this case, the form factor has a nontrivial dependence on the ratio $Q_{1}^{2} / Q_{2}^{2}$ of photon virtualities. Our calculation shows that the final result of our AdS/QCD model analytically coincides with the pQCD expression calculated using the asymptotic distribution amplitude $\varphi_{\pi}^{\text {as }}(x)=$ $6 x(1-x)$. This result is rather unexpected, because initial expressions for the form factor have very different structure. It should be noted that the absolute normalization of the form factor $K\left(Q_{1}^{2}, Q_{2}^{2}\right)$ in our model is fixed by adjusting its value to $K(0,0)=1$ at the real photon point, which allows us to conform to the QCD axial anomaly. The outcome that this choice exactly reproduces the leadingorder pQCD result for the equal-virtualities form factor $K\left(Q^{2}, Q^{2}\right)$ needs further studies, as well as our result that the $\omega$-dependence of the unequal-virtualities form factor $K\left((1+\omega) Q^{2},(1-\omega) Q^{2}\right)$ coincides with the leading-order pQCD result derived by assuming the asymptotic shape for the pion distribution amplitude.

The bulk-to-boundary propagators entering into AdS/QCD formulas for form factors have a generalized vector-meson-dominance (GVMD) decomposition. As a result, the form factors also can be written in GVMD form. We studied the interplay between the GVMD decomposition of form factors and their behavior for large photon virtualities. In the case of one real photon, the function $K\left(0, Q^{2}\right)$ asymptotically behaves like $1 / Q^{2}$. However, we demonstrated that this behavior has nothing to do with the fact that each term of the GVMD expansion for $K\left(0, Q^{2}\right)$ also behaves like $1 / Q^{2}$ for large $Q^{2}$. In fact, a formal GVMD expression for the coefficient of the $1 / Q^{2}$ term diverges. When both photons are highly virtual, each term of the GVMD expansion for $K\left(Q^{2}, Q^{2}\right)$ behaves like $1 / Q^{4}$, while $K\left(Q^{2}, Q^{2}\right)$ has $1 / Q^{2}$ asymptotic behavior. Thus, we observe that only in the region of small photon virtualities does it make sense to talk about the dominating role of the lowest states. In particular, for real photons, when $Q_{1}^{2}=Q_{2}^{2}=0$, the lowest (" $\rho \omega \pi$ ") transition amplitude contributes 1.15 into the $K(0,0)=1$ value, the excess being primarily cancelled by the neighboring non-diagonal transitions. 


\section{Chapter 6}

\section{Dimension Six Corrections to AdS/QCD Model}

\subsection{Introduction}

The significant progress of the holographic duals of QCD (based on [4]) in determination of basic hadronic observables (see, e.g., Refs. [26]-[59]) suggests further development. In this chapter*, we work in the vector sector of the AdS/QCD model with the hard-wall cutoff, proposed in the Ref. [26]. We study the effects of dimension six terms on the vector meson form factors and extract the values of observables such as the $\rho$-meson's electric radius, the mass, the decay constant, the magnetic and the quadrupole moments.

The leading order contribution to the vector meson form factors coming from the $F^{2}$ term has already been studied in detail in Refs. [37, 36], where it has been shown that the holographic models in Refs. $[26,32]$ reproduce only the trivial structure of vector mesons. In particular, instead of three independent form factors that describe vector meson, these holographic models predict only one.

We show that the inclusion of dimension six terms changes the situation towards a more interesting scenario in which all of the three form factors are corrected in different amounts. We also observe, that the only dimension six terms which give nontrivial contribution to the vector meson form factors are $X^{2} F^{2}$ and $F^{3}$. The contribution from the rest of the dimension six terms can be removed by the redefinition of the coupling constant $g_{5}^{2}$.

We find that the addition of a term such as $X^{2} F^{2}$ is equivalent to the AdS metric deformation and, according to Ref. [57], this, in turn, is equivalent to the inclusion of the vacuum condensates. This is in agreement with the point made in Ref. [26] that the higher dimension (HD) operators which appear in the operator product expansion of QCD arise in the holographic model from the higher terms in the 5D lagrangian such as $X^{2} F^{2}$. We also notice that the term $X^{2} F^{2}$ doesn't alter the values of the magnetic and the quadrupole moments, however, changes the values of the vector meson electric radius, the mass and the decay constant.

${ }^{*}$ The main results from the Ref. [39] are printed by permission from the Elsevier, see Appendix. 
This chapter is organized as follows, first we go through the basics of the holographic model, and in particular, discuss the leading order action, the equations of motion for the vector bound states and the forms of dimension six terms that can enter the action. Then, we demonstrate that a term like $X^{2} F^{2}$ doesn't change the values of the magnetic and the quadrupole moments and that its effect is equivalent to the AdS metric deformation. We also discuss, how this term, to a first approximation, changes the values of the $\rho$-meson mass, the decay constant and the electric charge radius. We consider the relevant part of the $F^{3}$ lagrangian and calculate the three-point function which is then used to derive the corrections to the form factors of vector mesons. Then, we calculate the charge radius, the magnetic and the quadrupole moments of the $\rho$-meson and compare these with the predictions from the other models given in Refs. [61]-[66]. Finally, we summarize and also show that the form factor of the pion can get corrections only from a term like $X^{2} F^{2}$.

\subsection{Preliminaries}

We are working in the background of the sliced AdS metric of the form:

$$
d s^{2}=\frac{1}{z^{2}}\left(\eta_{\mu \nu} d x^{\mu} d x^{\nu}-d z^{2}\right), \quad 0<z \leq z_{0}
$$

where $\eta_{\mu \nu}=\operatorname{Diag}(1,-1,-1,-1), z=z_{0}$ imposes the IR hard wall cutoff, (with $\left.z_{0} \sim 1 / \Lambda_{Q C D}\right)$ and $z=\epsilon \rightarrow 0$ determines the position of UV brane. From the dictionary of the AdS/QCD model, we will correspond to the $4 \mathrm{D}$ vector current $J_{\mu}^{a}(x)=\bar{q}(x) \gamma_{\mu} t^{a} q(x)$ a bulk gauge field $A_{\mu}^{a}(x, z)$ whose boundary value is the source for $J_{\mu}^{a}(x)$. The $5 \mathrm{D}$ gauge action in the $\mathrm{AdS}_{5}$ space is

$$
S_{\mathrm{AdS}}=-\frac{1}{4 g_{5}^{2}} \operatorname{Tr} \int d^{4} x d z \sqrt{g} F^{M N} F_{M N},
$$

where $F_{M N}=\partial_{M} A_{N}-\partial_{N} A_{M}-i\left[A_{M}, A_{N}\right], A_{M}=t^{a} A_{M}^{a}, \quad(M, N=0,1,2,3, z$; $\mu, \nu=0,1,2,3$ and $t^{a}=\sigma^{a} / 2$, where $\sigma^{a}$ are usual Pauli matrices with $\left.a=1,2,3\right)$. We work in the $A_{z}=0$ gauge and require $\partial_{\mu} A^{\mu}=0$.

Working in the Fourier image representation and defining $A_{\mu}^{a}(q, z)=A_{\mu}^{a}(q) A(q, z)$, we can determine the linearized equation of motion for $A(q, z)$, which is

$$
\left[z^{2} \partial_{z}^{2}-z \partial_{z}+q^{2} z^{2}\right] A(q, z)=0
$$

with boundary conditions $A(q, 0)=1$ and $\partial_{z} A\left(q, z_{0}\right)=0$.

In general, the $5 \mathrm{D}$ gauge theories are not renormalizable, since the $5 \mathrm{D}$ gauge coupling $g_{5}^{2}$ has negative mass dimension. This means that these theories can only be considered as an effective theories below some scale $\Lambda$. In particular, for our case, the cutoff scale $\Lambda$ should be set by $1 / g_{5}^{2}$.

Since, the holographic model is an effective theory with physical cutoff scale $\Lambda \sim 1 / g_{5}^{2}$, we are free to add HD terms into the lagrangian which respect all the required symmetries. The coefficients in front of the dimension six operators are 
of the form $c / \Lambda$, where $c$ is some dimensionless constant and $\Lambda=v / g_{5}^{2}$ (it can be estimated that $\left.v \sim 24 \pi^{3}\right)$. In general, since $g_{5}^{2}=12 \pi^{2} / N_{c}$, according to Ref. [26], we have $c / \Lambda=12 \pi^{2} c /\left(v N_{c}\right) \sim c / N_{c}$ and, therefore, for large $N_{c}$ the HD terms are $N_{c}$-suppressed.

There are three groups of dimension six terms one can add into the AdS/QCD lagrangian, which may contribute to the three-point function,

1. $\left(\nabla_{A} F_{M N}\right)^{2},\left(\nabla_{M} F^{M N}\right)^{2}$, $F^{3}, \quad F^{M N} \nabla^{2} F_{M N},\left(\nabla_{K} F^{M N}\right)\left(\nabla_{N} F_{M}^{K}\right)$,

2. $R F^{2}, R^{M N} F_{M K} F_{N}^{K}, R^{M N K P} F_{M N} F_{K P}$,

3. $X^{\dagger} X F F, X^{\dagger} F X F$,

where $\nabla_{M}$ is a covariant derivative, $R^{M N P K}, R^{M N}$ are Riemann and Ricci curvature tensors and $R$ is a Ricci scalar. Here, we will ignore the backreaction of the matter on the metric of the AdS space. As a result, the contribution from the terms of the second group becomes formal, since in the AdS space these terms are proportional to $F^{2}$ and can be absorbed into the coupling $g_{5}^{2}$.

Using the equation of motion

$$
\nabla_{M} F^{M N}=i\left[A_{K}, F^{K M}\right] \equiv J^{M}
$$

it can be shown that the term $\left(\nabla_{M} F^{M N}\right)^{2}$ doesn't contribute to the two-point and three-point functions. Notice, that the terms $F^{M N} \nabla^{2} F_{M N}$ and $\left(\nabla_{A} F_{M N}\right)^{2}$ are equivalent, since they differ by a full covariant derivative which vanishes after the integration because of the boundary conditions on the fields. The terms in the third group contribute to the three-point function in such a way that the magnetic and the quadrupole moments remain unchanged. We will show this on the example with the $X^{2} F^{2}$ term.

The remaining dimension six terms which can contribute to the three-point function are given in the second line of the first group. Using the properties of the covariant derivatives and the equation of motion, it can be shown that

$$
F^{M N} \nabla^{2} F_{M N} \supset 2 F^{M N} \nabla_{M} J_{N} \supset 2 \nabla_{M}\left(F^{M N} J_{N}\right)
$$

where we indicated only the parts which are not expressed through the terms in the second group or through the terms which don't contribute to the three-point function. The last term enters into the action as

$$
\operatorname{Tr} \int d^{5} x \sqrt{g} \nabla_{M}\left(F^{M N} J_{N}\right)=-i \operatorname{Tr} \int d^{4} x\left(\sqrt{g} F^{z \nu}\left[A^{\mu}, F_{\mu \nu}\right]\right)_{z=0} .
$$

It can be shown that this term doesn't contribute to the vector meson form factors. There are different ways to see this. One of the ways is, to notice, that the form factor is obtained as a double residue of the three-point function (see, e.g., Ref. [36]). Then, working in the Fourier image representation, we have $A(q, 0)=1$ and, therefore, the term $\left[A_{\mu}, F^{\mu \nu}\right]_{z=0}$ can't have any poles. As can be seen from the Eq. (6.25), only the 
$F^{z \nu}=A^{\nu}(q) \partial^{z} A(q, z=0)$ term in (6.6), has poles on the UV boundary. Therefore, since, we have only one term which has poles, the double residue will vanish, leading to zero corrections for the vector meson form factors. The similar arguments are applied for the term $\left(\nabla_{K} F^{M N}\right)\left(\nabla_{N} F_{M}^{K}\right)$. It appears, that only the term $F^{3}$ in this group can give non zero corrections to the form factors of vector mesons.

The terms of the first group $F^{M N} \nabla^{2} F_{M N}$ and $\left(\nabla_{K} F^{M N}\right)\left(\nabla_{N} F_{M}^{K}\right)$, contribute to the two-point function only through the terms in the second group. Therefore, the effect of these terms on the two point function is trivial and can be absorbed by the coupling $g_{5}^{2}$.

\subsection{The Effects From the $X^{2} F^{2}$ Term}

Consider the correction to the action (6.2), of the form

$$
S_{X^{2} F^{2}}=\kappa g_{5}^{2} \operatorname{Tr} \int d^{4} x d z \sqrt{g} X^{\dagger} X F^{M N} F_{M N},
$$

where $\kappa$ is some constant and following Ref. [26], we have $X^{2}=\mathbf{1}_{(2 \times 2)} v^{2}(z) / 4$. In particular, $v(z)=\left(m_{q} z+\sigma z^{3}\right)$, where $m_{q}$ is the quark mass parameter and $\sigma$ plays the role of the chiral condensate.

We observe that the total action can be written as

$$
S_{F^{2}}+S_{X^{2} F^{2}}=-\frac{1}{4 g_{5}^{2}} \operatorname{Tr} \int d^{4} x d z \frac{p(z)}{z} F^{M N} F_{M N},
$$

where the Lorentz indexes are now governed by the flat metric $\eta_{M N}, p(z)=1-$ $\kappa g_{5}^{4} v^{2}(z)$ and it is clear that, in general, the contribution from all the terms like $X^{2 n} F^{2}$, ( $n$ is natural number), will modify $p(z)$ to a function $P(v(z)) \equiv 1+$ $C_{1} g_{5}^{4} v^{2}(z)+\cdots+C_{n} g_{5}^{4 n} v^{2 n}(z)$, where $C_{n}$ are some unknown coefficients. Therefore, the inclusion of the $X^{2} F^{2}$ term corresponds effectively to the deformation of the AdS metric, that is instead of the $1 / z^{2}$ factor in the metric (6.1), we will have $p^{2}(z) / z^{2}$. The similar arguments are applied also for the term $X^{\dagger} F X F$.

This observation allows the direct application of the result from the Ref. [36] to the present case, leaving us with the following expression for the elastic form factors:

$$
\tilde{F}_{n n}\left(Q^{2}\right)=\int_{0}^{z_{0}} d z \frac{p(z)}{z} \mathcal{J}(Q, z)\left|\psi_{n}(z)\right|^{2},
$$

where $\psi_{n}(z)$ are the solutions of the equations of motion,

$$
\partial_{z}\left[\frac{p(z)}{z} \partial_{z} \psi_{n}(z)\right]+\frac{p(z)}{z} M_{n}^{2} \psi_{n}(z)=0,
$$

with b.c. $\psi_{n}(0)=\psi_{n}^{\prime}\left(z_{0}\right)=0$ and $q^{2}=M_{n}^{2}$. The function $\mathcal{J}(Q, z)$ is a solution of the same equation of motion but with $q^{2}=-Q^{2}$ instead of $M_{n}^{2}$ and b.c. $\mathcal{J}(Q, 0)=1$, $\partial_{z} \mathcal{J}\left(Q, z_{0}\right)=0$. The eigenfunctions of Eq. (6.9) are normalized as

$$
\int_{0}^{z_{0}} d z \frac{p(z)}{z}\left|\psi_{n}(z)\right|^{2}=1
$$


Therefore, $\tilde{F}_{n n}(0)=1$ and, since, the electric $G_{C}$, magnetic $G_{M}$ and quadrupole $G_{Q}$ form factors are:

$$
\begin{aligned}
& G_{Q}^{(n)}\left(Q^{2}\right)=-\tilde{F}_{n n}\left(Q^{2}\right), \quad G_{M}^{(n)}\left(Q^{2}\right)=2 \tilde{F}_{n n}\left(Q^{2}\right) \\
& G_{C}^{(n)}\left(Q^{2}\right)=\left(1-\frac{Q^{2}}{6 M_{n}^{2}}\right) \tilde{F}_{n n}\left(Q^{2}\right)
\end{aligned}
$$

one can check that at $Q^{2}=0$, these form factors reproduce the same values for electric charge, magnetic and quadrupole moments, as in the case with $\kappa=0$, that is in the absence of the $X^{2} F^{2}$ term. This term, however, can change masses and decay constants of vector mesons. Besides, it also changes the electric radius of the $\rho$-meson.

Notice, that the eigenvalues of the Eq. (6.9) may be expressed through the eigenfunctions in the following way:

$$
M_{n}^{2}=\int_{0}^{z_{0}} d z \frac{p(z)}{z}\left|\partial_{z} \psi_{n}(z)\right|^{2} .
$$

Up to a first order approximation, using the same eigenfunctions as in case with $\kappa=0$, that is

$$
\psi_{n}^{(0)}(z)=\frac{\sqrt{2}}{z_{0} J_{1}\left(\gamma_{0, n}\right)} z J_{1}\left(M_{n}^{(0)} z\right)
$$

with $M_{n}^{(0)}=\gamma_{0, n} / z_{0}\left(\right.$ where $\left.J_{0}\left(\gamma_{0, n}\right)=0\right)$ but with metric perturbation $p(z)$, we will have for the $\rho$-meson mass $M_{\rho} \equiv M_{1}$ the following result:

$$
M_{\rho} \simeq M_{\rho}^{(0)}\left(1-0.02 \kappa g_{5}^{4}\right)
$$

where $M_{\rho}^{(0)}$ is the mass of the $\rho$-meson in case $\kappa=0$, and we used the values of parameters: $m_{q}=2.3 \mathrm{MeV}, \sigma=(327 \mathrm{MeV})^{3}, z_{0}=1 /(323 \mathrm{MeV})$, taken from the Model A of Ref. [26].

The decay constant of the $\rho$-meson, $f_{\rho}$, in terms of the eigenfunctions of the $5 \mathrm{D}$ equation of motion has the form

$$
f_{\rho}=\frac{1}{g_{5}}\left(\frac{p(z)}{z} \partial_{z} \psi_{\rho}(z)\right)_{z \rightarrow 0},
$$

as was discussed, for example, in the Ref. [37]. The solution for $\psi_{\rho}(z) \equiv \psi_{1}(z)$ near the $z=0$ is of the same form as in case $\kappa=0$ thus,

$$
f_{\rho}=\frac{\sqrt{2} M_{\rho}}{g_{5} z_{0} J_{1}\left(\gamma_{0,1}\right)} .
$$

Therefore, to lowest order in $\kappa$, we will have:

$$
f_{\rho} \simeq f_{\rho}^{(0)}\left(1-0.02 \kappa g_{5}^{4}\right)
$$


where $f_{\rho}^{(0)}$ is the decay constant in case when $\kappa=0$.

We can also express the electric charge radius of the $\rho$-meson, $\left\langle\tilde{r}_{\rho}^{2}\right\rangle_{C}$, defined as

$$
\left\langle\tilde{r_{\rho}^{2}}\right\rangle_{C} \equiv-6\left(\frac{d G_{C}^{(1)}\left(Q^{2}\right)}{d Q^{2}}\right)_{Q^{2}=0},
$$

in terms of the parameter $\kappa$. In this case, using the Eqs. (6.8), (6.11) and (6.18), to lowest order in the coefficient $\kappa$, the electric charge radius is:

$$
\left\langle\tilde{r}_{\rho}^{2}\right\rangle_{C} \simeq\left(0.53-0.16 \kappa g_{5}^{4}\right) \mathrm{fm}^{2},
$$

where $0.53 \mathrm{fm}^{2}$ is the result for the electric radius obtained in Ref. [36] (again, we used parameters taken from the Model A of Ref. [26]).

The similar analysis can be applied for the case of Model B in Ref. [26], for which we have:

$$
\begin{aligned}
M_{\rho} & \simeq M_{\rho}^{(0)}\left(1-0.01 \kappa g_{5}^{4}\right), \\
f_{\rho} & \simeq f_{\rho}^{(0)}\left(1-0.01 \kappa g_{5}^{4}\right), \\
\left\langle\tilde{r}_{\rho}^{2}\right\rangle_{C} & \simeq\left(0.46-0.07 \kappa g_{5}^{4}\right) \mathrm{fm}^{2} .
\end{aligned}
$$

Notice, that the coefficients in front of $\kappa$, in the case of Model B are almost twice as small as that in the Model A. Also, it is straightforward to see that the contribution from the term $X^{\dagger} F X F$ can be absorbed by $\kappa$.

Now, since $g_{5}^{2}=12 \pi^{2} / N_{c}$, it follows that the corrections to the observables $\left(\sim \kappa g_{5}^{4}\right)$ are $1 / N_{c}^{2}$ suppressed. The natural constraint on the coefficient $\kappa$ should come from the requirement that the corrections to the observables are small. This means that, if $N_{c}=3$, then for the first two observables in (6.20), we should have $|\kappa| \ll 0.06$ and for the third one we expect to have $|\kappa| \ll 0.004$. Therefore, we conclude, that it is natural for the coefficient $\kappa$ to satisfy the condition $|\kappa| \ll 10^{-3}$.

\subsection{Corrections From the $F^{3}$ Term}

The action relevant for finding the corrections to the 3-point function is

$$
\begin{aligned}
S_{F^{3}} & =\frac{1}{2} \alpha g_{5}^{2} \operatorname{Tr} \int d^{4} x d z \sqrt{g}\left(\left[F_{M N}, F^{N K}\right] F_{K}{ }^{M}\right) \\
& \supset \frac{i \alpha g_{5}^{2} \epsilon^{a b c}}{4} \int d^{4} x d z z\left[3\left(\partial_{\mu} A_{\nu}^{a}\right)\left(\partial_{z} A^{b, \nu}\right)\left(\partial_{z} A^{c, \mu}\right)+2\left(\partial^{\mu} A^{a, \nu}\right) F_{\nu}^{b, \alpha} F_{\alpha \mu}^{c}\right],
\end{aligned}
$$

where $\alpha$ is a new dimensionless parameter of the theory and the Lorentz indexes are governed by the Minkowski flat metric $\eta_{\mu \nu}$. Therefore, using the prescription of the holographic model, for the 3-point function we will have:

$$
\begin{aligned}
T_{\mu \alpha \beta}^{a b c}\left(p_{1}, p_{2}, q\right) & \equiv\left\langle J_{\alpha}^{b}\left(p_{1}\right) J_{\mu}^{a}(q) J_{\beta}^{c}\left(-p_{2}\right)\right\rangle \\
& =\epsilon^{a b c} T_{\mu \alpha \beta}\left(p_{1}, p_{2}, q\right) i(2 \pi)^{4} \delta^{(4)}\left(q-p_{2}+p_{1}\right),
\end{aligned}
$$


where

$$
\begin{aligned}
T_{\mu \alpha \beta}\left(p_{1}, p_{2}, q\right) & =\frac{3 \alpha g_{5}^{2}}{4}\left\{\left[q^{2} K_{2}-K_{11}\right] \eta_{\alpha \beta}\left(p_{1}+p_{2}\right)_{\mu}\right. \\
& +\left[2 M^{2} K_{2}-K_{12}\right]\left(\eta_{\mu \alpha} q_{\beta}-\eta_{\mu \beta} q_{\alpha}\right) \\
& \left.-2 K_{2} q_{\alpha} q_{\beta}\left(p_{1}+p_{2}\right)_{\mu}\right\}
\end{aligned}
$$

and

$$
\begin{aligned}
K_{11}\left(p_{1}, p_{2}, q\right) & =\int_{0}^{z_{0}} d z z \partial_{z} A(q, z) A\left(p_{1}, z\right) \partial_{z} A\left(p_{2}, z\right), \\
K_{12}\left(p_{1}, p_{2}, q\right) & =\int_{0}^{z_{0}} d z z \partial_{z}\left[A(q, z) A\left(p_{1}, z\right)\right] \partial_{z} A\left(p_{2}, z\right), \\
K_{2}\left(p_{1}, p_{2}, q\right) & =\int_{0}^{z_{0}} d z z A(q, z) A\left(p_{1}, z\right) A\left(p_{2}, z\right),
\end{aligned}
$$

where we used that the functions $K\left(p_{1}, p_{2}, q\right)$ are symmetric under the exchange of $p_{1} \leftrightarrow p_{2}$ (to understand this, see Eq. (6.25)), but not $p_{1,2} \leftrightarrow q,\left(q=p_{2}-p_{1}\right)$ and anticipating the on-shell limit, we applied conditions: $p_{1}^{2}=p_{2}^{2}=M^{2},\left(p_{1} p_{2}\right)=$ $M^{2}-q^{2} / 2$ and $\left(p_{2} q\right)=-\left(p_{1} q\right)=q^{2} / 2$, for the diagonal transitions (one can easily generalize this to non diagonal transition). Since we are dealing with the transverse components of the gauge field, to simplify the tensor structure, we applied, as in [37], the transverse projectors $\Pi^{\alpha \alpha^{\prime}}\left(p_{1}\right) \equiv\left(\eta^{\alpha \alpha^{\prime}}-p_{1}^{\alpha} p_{1}^{\alpha^{\prime}} / p_{1}^{2}\right)$, etc, (that allows us to add or eliminate terms proportional to $p_{1 \alpha}$ or $\left.p_{2 \beta}\right)$. The solution of the (6.3) for timelike momentum can be written as an infinite sum:

$$
A(p, z)=-g_{5} \sum_{m=1}^{\infty} \frac{f_{m} \psi_{m}(z)}{p^{2}-M_{m}^{2}}
$$

where $\psi_{m}(z)$ are the solutions of the (6.3) with b.c. $\psi_{m}(0)=\psi_{m}^{\prime}\left(z_{0}\right)=0$ and $q^{2}=M_{m}^{2}$. Then, for a spacelike momentum transfer, $q^{2}=-Q^{2}$, it follows that:

$$
T_{\mu \alpha \beta}\left(p_{1}, p_{2}, q\right)=\frac{3 \alpha g_{5}^{4}}{4} \sum_{n, k=1}^{\infty} \frac{f_{m} f_{n} R_{\mu \alpha \beta}^{n k}\left(Q^{2}\right)}{\left(p_{1}^{2}-M_{n}^{2}\right)\left(p_{2}^{2}-M_{k}^{2}\right)}
$$

and for the diagonal $n \leftrightarrow n$ transition:

$$
\begin{aligned}
R_{\mu \alpha \beta}^{(n)}\left(Q^{2}\right) & \equiv \lim _{p_{1}^{2} \rightarrow M_{n}^{2}} \lim _{p_{2}^{2} \rightarrow M_{n}^{2}}\left(p_{1}^{2}-M_{n}^{2}\right)\left(p_{2}^{2}-M_{n}^{2}\right) T_{\mu \alpha \beta} \\
& =\frac{3 \alpha g_{5}^{4}}{4}\left\{-\left[Q^{2} W_{2}^{n n}+W_{11}^{n n}\right] \eta_{\alpha \beta}\left(p_{1}+p_{2}\right)_{\mu}\right. \\
& +\left[2 M_{n}^{2} W_{2}^{n n}-W_{12}^{n n}\right]\left(\eta_{\mu \alpha} q_{\beta}-\eta_{\mu \beta} q_{\alpha}\right) \\
& \left.-2 W_{2}^{n n} q_{\alpha} q_{\beta}\left(p_{1}+p_{2}\right)_{\mu}\right\}
\end{aligned}
$$


where we defined new functions as

$$
\begin{aligned}
& W_{11}^{n n}\left(Q^{2}\right)=\int_{0}^{z_{0}} d z z \partial_{z} \mathcal{J}(Q, z) \psi_{n}(z) \partial_{z} \psi_{n}(z) \\
& W_{12}^{n n}\left(Q^{2}\right)=\int_{0}^{z_{0}} d z z \partial_{z}\left[\mathcal{J}(Q, z) \psi_{n}(z)\right] \partial_{z} \psi_{n}(z), \\
& W_{2}^{n n}\left(Q^{2}\right)=\int_{0}^{z_{0}} d z z \mathcal{J}(Q, z) \psi_{n}(z) \psi_{n}(z) .
\end{aligned}
$$

with

$$
\mathcal{J}(Q, z)=Q z\left[K_{1}(Q z)+I_{1}(Q z) \frac{K_{0}\left(Q z_{0}\right)}{I_{0}\left(Q z_{0}\right)}\right]
$$

where $\mathcal{J}(Q, z)=A(Q, z)$ is the solution of Eq. (6.3).

\subsection{Form Factors}

Adding the corrections to the form factor coming from the $F^{3}$ term to the leading order result from the $F^{2}$ term obtained in Ref. [36] gives for the electric $\tilde{G}_{C}$, magnetic $\tilde{G}_{M}$ and quadrupole $\tilde{G}_{Q}$ form factors the following result

$$
\begin{aligned}
\tilde{G}_{C}^{(n)}\left(Q^{2}\right) & =\left[1-\frac{Q^{2}}{6 M_{n}^{2}}\right] F_{n n}-\frac{3 \alpha g_{5}^{4} Q^{2}}{4}\left[1+\frac{Q^{2}}{12 M_{n}^{2}}\right] W_{2}^{n n} \\
& -\frac{3 \alpha g_{5}^{4}}{4}\left[1+\frac{Q^{2}}{6 M_{n}^{2}}\right] W_{11}^{n n}+\frac{\alpha g_{5}^{4} Q^{2}}{8 M_{n}^{2}} W_{12}^{n n} \\
\tilde{G}_{M}^{(n)}\left(Q^{2}\right) & =2 F_{n n}\left(Q^{2}\right)+\frac{3 \alpha g_{5}^{4}}{4}\left[2 M_{n}^{2} W_{2}^{n n}-W_{12}^{n n}\right] \\
\tilde{G}_{Q}^{(n)}\left(Q^{2}\right) & =-F_{n n}\left(Q^{2}\right)-\frac{3 \alpha g_{5}^{4} Q^{2}}{8} W_{2}^{n n} \\
& -\frac{3 \alpha g_{5}^{4}}{4}\left[W_{11}^{n n}-W_{12}^{n n}\right] .
\end{aligned}
$$

where

$$
F_{n n}\left(Q^{2}\right)=\int_{0}^{z_{0}} \frac{d z}{z} \mathcal{J}(Q, z)\left|\psi_{n}(z)\right|^{2}
$$

see Ref. [36] for more details. In the AdS/QCD model, with $\alpha=0$ as was shown in [36], these three form factors of vector meson are expressed through the single function $F_{n n}\left(Q^{2}\right)$. Besides, for $Q^{2}=0$, the AdS/QCD model reproduces the unit electric charge $e$ of the meson, "predicts" $\mu \equiv G_{M}(0)=2$ for the magnetic moment and $D \equiv G_{Q}(0) / M^{2}=-1 / M^{2}$ for the quadrupole moment, which are just the canonical values for a vector particle [60]. However, for non zero values of $\alpha$ the situation changes towards a more realistic scenario. 


\subsection{Results}

One can verify that at $Q^{2}=0$, we have $W_{11}^{n n}(0)=0$, because $\partial_{z} \mathcal{J}(0, z)=0$, since

$$
\partial_{z} \mathcal{J}(Q, z)=-z Q^{2}\left[K_{0}(Q z)-I_{0}(Q z) \frac{K_{0}\left(Q z_{0}\right)}{I_{0}\left(Q z_{0}\right)}\right] .
$$

In addition

$$
\begin{aligned}
& W_{12}^{11}(0)=\int_{0}^{z_{0}} d z z\left(\partial_{z} \psi_{1}(z)\right)^{2}=\frac{2 M^{2} z_{0}^{2}}{J_{1}^{2}\left(\gamma_{0,1}\right)} \int_{0}^{1} d \zeta \zeta^{3} J_{0}^{2}\left(\gamma_{0,1} \zeta\right), \\
& W_{2}^{11}(0)=\int_{0}^{z_{0}} d z z \psi_{1}^{2}(z)=\frac{2 z_{0}^{2}}{J_{1}^{2}\left(\gamma_{0,1}\right)} \int_{0}^{1} d \zeta \zeta^{3} J_{1}^{2}\left(\gamma_{0,1} \zeta\right),
\end{aligned}
$$

where $J_{0}\left(\gamma_{0,1}\right)=0, M=\gamma_{0,1} / z_{0}$ is the mass of the $\rho$-meson and we took into account that

$$
\psi_{1}(z)=\frac{\sqrt{2}}{z_{0} J_{1}\left(\gamma_{0,1}\right)} z J_{1}(M z)
$$

After partial integrations and using the properties of Bessel functions we obtain

$$
W_{12}^{11}(0)=M^{2} W_{2}^{11}(0)-2 .
$$

Now, defining $w \equiv W_{12}^{11}(0) \simeq 1.261$, we find $(e=1)$,

$$
\begin{aligned}
\mu & \equiv \tilde{G}_{M}^{(1)}(0)=2+\frac{3 \alpha g_{5}^{4}}{4}(w+4), \\
D M^{2} & \equiv \tilde{G}_{Q}^{(1)}(0)=-1+\frac{3 \alpha g_{5}^{4} w}{4} .
\end{aligned}
$$

The electric radius of the $\rho$-meson is

$$
\begin{aligned}
\left\langle\tilde{r}_{\rho}^{2}\right\rangle_{C} & \equiv-6\left(\frac{d \tilde{G}_{C}^{(1)}\left(Q^{2}\right)}{d Q^{2}}\right)_{Q^{2}=0} \\
& =\left\langle r_{\rho}^{2}\right\rangle_{C}+\alpha g_{5}^{4}\left[\frac{3}{4 M^{2}}(5 w+12)+\frac{9}{2}\left(\frac{d W_{11}^{11}\left(Q^{2}\right)}{d Q^{2}}\right)_{Q^{2}=0}\right]
\end{aligned}
$$

where the first term is $\left\langle r_{\rho}^{2}\right\rangle_{C}=0.53 \mathrm{fm}^{2}$, found in Ref. [36], and the second term in the square brackets is the correction to the $\rho$-meson's radius. Using Eqs. (6.27), (6.33) and (6.36) one can find that

$$
\frac{9}{2}\left(\frac{d W_{11}^{11}\left(Q^{2}\right)}{d Q^{2}}\right)_{Q^{2}=0}=\frac{9 \gamma_{0,1} z_{0}^{2}}{J_{1}^{2}\left(\gamma_{0,1}\right)} \int_{0}^{1} d \zeta \zeta^{4} \ln \zeta J_{0}\left(\gamma_{0,1} \zeta\right) J_{1}\left(\gamma_{0,1} \zeta\right)
$$

which is $\simeq-0.255 \mathrm{fm}^{2}$. Therefore,

$$
\sigma \equiv\left(\left\langle\tilde{r}_{\rho}^{2}\right\rangle_{C}-\left\langle r_{\rho}^{2}\right\rangle_{C}\right) / \mathrm{fm}^{2} \simeq 0.647 \alpha g_{5}^{4} \simeq 252 \alpha
$$


Now, in terms of $\sigma$, the magnetic and quadrupole moments of the $\rho$-meson are: $\mu \simeq 2+6.1 \sigma$ and $D M^{2}=1.46 \sigma-1$. The table of possible values for electric radius, magnetic and quadrupole moments in terms of a reasonable range of values for $\sigma$ is given below:

TABLE I: The observables for different values of $\sigma$.

\begin{tabular}{|c|c|c|c|c|c|c|c|c|}
\hline$\sigma$ & -0.15 & -0.1 & -0.05 & -0.01 & 0.01 & 0.05 & 0.1 & 0.15 \\
\hline \hline$r^{2}$ & 0.38 & 0.43 & 0.48 & 0.52 & 0.54 & 0.58 & 0.63 & 0.68 \\
$\mu$ & 1.09 & 1.39 & 1.7 & 1.94 & 2.06 & 2.31 & 2.61 & 2.92 \\
$-D M^{2}$ & 1.22 & 1.15 & 1.07 & 1.01 & 0.99 & 0.93 & 0.85 & 0.78 \\
\hline
\end{tabular}

where $r^{2} \equiv\left\langle\tilde{r_{\rho}^{2}}\right\rangle_{C} / \mathrm{fm}^{2}$. These results depend explicitly on $\alpha$ (or $\sigma$ ) and implicitly on $z_{0}$ which is fixed by the mass of the $\rho$-meson. Notice, that $g_{5}^{4}|\alpha|<0.23$, therefore, we are not outside of the perturbative domain and our calculations are consistent. For comparison with other models, see table below

TABLE II: The observables in different models.

\begin{tabular}{|c|c|c|c|c|c|c|}
\hline Models & {$[61]$} & {$[62]$} & {$[63]$} & {$[64]$} & {$[65]$} & {$[66]$} \\
\hline \hline$r^{2}$ & 0.27 & 0.37 & 0.37 & 0.39 & 0.54 & 0.55 \\
$\mu$ & 1.92 & 2.69 & 2.14 & 2.48 & 2.01 & 2.25 \\
$-D M^{2}$ & 0.43 & 0.84 & 0.79 & 0.89 & 0.41 & 0.11 \\
\hline
\end{tabular}

It is interesting, that the only HD term in the 5D effective theory that can alter the canonical values of the magnetic and the quadrupole moments is the term $F^{3}$. Therefore, the more precise knowledge of either one of these observables $(\mu, D$ or $\left.r^{2}\right)$ can put more stringent constraints on the coefficient $\alpha$. Here, we showed that the corrections are proportional to $\alpha g_{5}^{4}$ and thus, are $1 / N_{c}^{2}$ suppressed as expected. Finally, our estimates suggest that $|\alpha|<10^{-4}$.

\subsection{Summary}

In this chapter, as one of the possible ways to test and improve the AdS/QCD model proposed in the Ref. [26], we considered the addition of dimension six terms into the vector sector of the AdS/QCD lagrangian and studied their effect on the vector meson form factors.

We discussed that ignoring the backreaction of the matter on the metric, the effect from the terms of the second group involving the AdS curvature tensors and Ricci scalar, is equivalent to the redefinition of the coupling $g_{5}^{2}$. We showed that a term, like $X^{2} F^{2}$, doesn't change the electric charge, the magnetic and the quadrupole moments, but affects the charge radius, the masses and the decay constants of the vector mesons. The effect of this term is equivalent to the AdS metric deformation and, in agreement with [26] and [57], it is also equivalent to the addition of vacuum condensates. However, one should keep in mind that the metric deformations are also coming from the matter fields, which we ignore compared to the explicit or effective metric deformations from the $X^{2} F^{2}$ term. 
By calculating the form factors, we found a relation between electric charge radius, mass, decay constant of the $\rho$-meson and the coefficient $\kappa$ (to lowest order) with which the term $X^{2} F^{2}$ enters the action. Also, we expressed the electric radius, magnetic and quadrupole moments of the $\rho$-meson in terms of the dimensionless parameter $\alpha$, with which the term $F^{3}$ enters the action. These results can be straightforwardly generalized to the case of the soft wall model $[32,37]$.

It is also interesting to study the contribution of the dimension six terms to the form factor of pion. As discussed in Ref. [38], in the full AdS/QCD model the pion form factor is derived from the variation of the action with respect to the two longitudinal axial-vector fields and one transverse vector field. As a result, only terms like $X^{2} F^{2}$ can contribute to the form factor of pion. To demonstrate this, first, consider the term $F_{A}^{2} F_{V}$, where $F_{A}$ is related to the axial-vector field. This term may contribute to the three-point function in such a way that only the linear pieces of the field strength tensors can enter. However, since these linear pieces vanish for the longitudinal axial-vector field, there can't be any contribution from term like $F^{3}$ to the form factor of pion (this question was also discussed in Ref. [59]).

The other relevant dimension six terms $\left(\nabla_{A} F_{M N}\right)^{2}$ and $\left(\nabla_{K} F^{M N}\right)\left(\nabla_{N} F_{M}^{K}\right)$ also can't contribute to the form factor of pion. We demonstrate this for example with the term $\left(\nabla_{A} F_{M N}\right)^{2}$ which, as shown above contributes to the action in the form given in Eq. (6.6). However, this term contains two field strength tensors, and at least one should vanish for the longitudinal components. Similar arguments can be also applied for the second term.

Finally, we think that the results obtained here are in the range of the values from the other models. This is encouraging and suggests that the further addition of the HD terms can improve the holographic dual model of QCD. 


\section{Chapter 7}

\section{Conclusion}

In 1997, Maldacena conjectured a surprising connection between the QCD-like theory $^{*}$ in four-dimensions and the gravity theory ${ }^{\dagger}$ which lives in the warped fivedimensional AdS space ${ }^{\ddagger}$. This conjecture is known as the AdS/CFT correspondence. While CFT is similar to QCD, there are some differences that are partially fixed in the so-called holographic dual model of $Q C D$ or the $A d S / Q C D$ model, where one roughly makes the size of the extra fifth dimension finite. This method allows one to solve nonperturbative problems of QCD by applying well known perturbative techniques to a 5D theory. This is exciting and relevant to strong interaction physics, since we are now able to understand QCD at large distances where the strong forces start to play an important role. Using this method we can calculate the masses of hadrons and many other observables which couldn't be calculated otherwise in perturbation theory. It is as if the hadronic physics in 4D appears as the holographic image of the theory which lives in 5D.

To demonstrate the effectiveness of the AdS/QCD model, we have calculated some of the important hadronic observables which could be compared either with experiment or with lattice QCD data. As a result, using a framework of AdS/QCD model with hard-wall cutoff, we developed a formalism to calculate hadronic observables such as form factors and wave functions of vector mesons. We showed that these form factors manifest a generalized vector-meson dominance (VMD) representation with a very specific VMD pattern, in which form factors are essentially given by contributions due to the first two bound states. The electric radius of the rho-meson from the holographic model was shown to be in a good agreement with predictions from lattice QCD.

In chapter 3, we studied wave functions and form factors of vector mesons within the framework of the soft-wall model, that can produce a Regge-like spectrum for higher radially excited mesons, expected from semi-classical arguments. Analyzing the three-point function, we obtained expressions for transition form factors both in terms of the $\psi$ wave functions and the "more physical" $\Phi$ wave functions, which are simply Laguerre polynomials. We showed that, just like in the hard-wall model, the

* More precisely strongly coupled conformal field theory or CFT for short.

$\dagger$ Weakly coupled, type IIB string theory, to be precise.

${ }^{\ddagger}$ AdS stands for Anti-de Sitter space which has constant negative curvature. 
form factors in the soft-wall model can be written in the form of generalized VMD representation, i.e., as a sum over all bound states in the channel of electromagnetic current. We also calculated the $\rho$-meson electric radius in the soft-wall model, and found that it is larger than in the hard-wall model. Finally, we calculated the $\rho$ meson coupling constant $f_{\rho}$ both in the soft-wall and hard-wall models, and found that the experimental value is closer to the hard-wall model result.

In Chapter 4, using the AdS/QCD model, we studied the pion in the chiral limit of QCD with two flavors. We described a formalism that allows one to extract the pion form factor, where we identified the pion with the longitudinal component of the axial-vector gauge field. We found an analytic expression for the pion decay constant in terms of two parameters of the model: $\sigma$ and $z_{0}$. Analyzing the results, we found it convenient to work with two combinations $\alpha=g_{5} \sigma / 3$ and $a=\alpha z_{0}^{3}$ of the basic parameters. In particular, we found $a=a_{0}=2.26$ for the value of $a$ corresponding to the experimental $\rho$-meson mass $m_{\rho}$ and pion decay constant $f_{\pi}$. The importance of the parameter $a$ is that its magnitude determines the regions, where the pion properties are either governed by the confinement effects or by the effects from the spontaneous chiral symmetry breaking. We also found that the pion charge radius $\left\langle r_{\pi}^{2}\right\rangle^{1 / 2} \approx 0.58 \mathrm{fm}$ in the hard-wall model is smaller than that measured experimentally. The basic source of the discrepancy with experiment comes from the fact that the asymptotic AdS/QCD prediction for the pion form factor is $Q^{2} F_{\pi}\left(Q^{2}\right) \rightarrow 4 \pi^{2} f_{\pi}^{2}$, and if one takes the experimental value for $f_{\pi}$, one obtains $Q^{2} F_{\pi}\left(Q^{2}\right) \rightarrow 0.68 \mathrm{GeV}^{2}$, which is much larger than the $0.4 \mathrm{GeV}^{2}$ value given by $Q^{2} \sim 2 \mathrm{GeV}^{2}$ JLab data.

In Chapter 5, we developed an extension of the AdS/QCD model, adjusted to study the anomalous coupling of the neutral pion to two (in general, virtual) photons. The additional part of the gauge field in the $5 \mathrm{D}$ bulk is associated with the isoscalar vector current (related to $\omega$-like mesons). The Chern-Simons term allows one to reproduce the tensor structure of the anomalous form factor $F_{\gamma^{*} \gamma^{*} \pi^{0}}\left(Q_{1}^{2}, Q_{2}^{2}\right)$. To exactly reproduce the QCD anomaly result for real photons, we added contributions localized at the IR boundary $z=z_{0}$, and then studied the momentum dependence of the $F_{\gamma^{*} \gamma^{*} \pi^{0}}\left(Q_{1}^{2}, Q_{2}^{2}\right)$ form factor in our model. In particular, we calculated the slope of the form factor with one real and one slightly off-shell photon. Our result $a_{\pi} \approx 0.031$ is very close to the value $a_{\pi}=0.0326 \pm 0.0026$ obtained by CELLO collaboration from spacelike $Q^{2}$ measurements.

In chapter 6 , as one of the possible ways to test and improve the AdS/QCD model, we considered the addition of dimension six terms into the vector sector of the AdS/QCD lagrangian and studied their effect on the vector meson form factors. We showed that terms like $X^{2} F^{2}$ don't change the electric charge, the magnetic and the quadrupole moments, but do affect the charge radius, the masses and the decay constants of the vector mesons. The effect of this term is equivalent to the AdS metric deformation and is also equivalent to the addition of the vacuum condensates. We also expressed the electric radius, magnetic and quadrupole moments of the $\rho$ meson in terms of the parameter with which the other dimension six term $F^{3}$ enters the action. 


\section{Bibliography}

[1] D. J. Gross and F. Wilczek, "Asymptotically Free Gauge Theories," Phys. Rev. D 8, 3633 (1973); Phys. Rev. D 9, 980 (1974); H. D. Politzer, "Reliable Perturbvative Results for Strong Interactions?" Phys. Rev. Lett. 30, 1346 (1973); "Asymptotic Freedom: An Approach To Strong Interactions," Phys. Rept. 14, 129 (1974).

[2] T. DeGrand and C. E. Detar, "Lattice methods for quantum chromodynamics," New Jersey, USA: World Scientific (2006) 345 p.

[3] C. D. Roberts and A. G. Williams, "Dyson-Schwinger equations and their application to hadronic physics," Prog. Part. Nucl. Phys. 33, 477 (1994).

[4] J. M. Maldacena, "The large N limit of superconformal field theories and supergravity," Adv. Theor. Math. Phys. 2, 231 (1998) [Int. J. Theor. Phys. 38, 1113 (1999)]; S. S. Gubser, I. R. Klebanov and A. M. Polyakov, "Gauge theory correlators from non-critical string theory," Phys. Lett. B 428, 105 (1998); E. Witten, "Anti-de Sitter space and holography," Adv. Theor. Math. Phys. 2, 253 (1998).

[5] O. Aharony, S. S. Gubser, J. M. Maldacena, H. Ooguri and Y. Oz, "Large N field theories, string theory and gravity," Phys. Rept. 323, 183 (2000).

[6] B. Zwiebach, "A first course in string theory," Cambridge, UK: Univ. Pr. (2004) $558 \mathrm{p}$.

[7] M. B. Green, J. H. Schwarz and E. Witten, "Superstring Theory. Vol. 1: Introduction," Cambridge, Uk: Univ. Pr. (1987) 469 p.; "Superstring Theory. Vol. 2: Loop Amplitudes, Anomalies And Phenomenology," Cambridge, Uk: Univ. Pr. (1987) 596 p. (Cambridge Monographs On Mathematical Physics).

[8] J. Polchinski, "String theory. Vol. 1: An introduction to the bosonic string," Cambridge, UK: Univ. Pr. (1998) 402 p; "String theory. Vol. 2: Superstring theory and beyond," Cambridge, UK: Univ. Pr. (1998) 531 p.

[9] K. Becker, M. Becker and J. H. Schwarz, "String theory and M-theory: A modern introduction," Cambridge, UK: Cambridge Univ. Pr. (2007) 739 p.

[10] R. Dolen, D. Horn and C. Schmid, "Finite energy sum rules and their application to pi N charge exchange," Phys. Rev. 166 (1968) 1768. 
[11] G. Veneziano, "Construction of a crossing - symmetric, Regge behaved amplitude for linearly rising trajectories," Nuovo Cim. A 57 (1968) 190.

[12] Y. Nambu, "QCD And The String Model," Phys. Lett. B 80, 372 (1979).

[13] H. B. Nielsen, submitted to the 15th International Conference on High Energy Physics, Kiev (1970).

[14] L. Susskind, "Dual Symmetric Theory Of Hadrons. 1," Nuovo Cim. A 69 (1970) 457.

[15] I. R. Klebanov, "QCD and string theory," Int. J. Mod. Phys. A 21, 1831 (2006).

[16] M. Luscher, K. Symanzik and P. Weisz, "Anomalies Of The Free Loop Wave Equation In The Wkb Approximation," Nucl. Phys. B 173, 365 (1980).

[17] M. Luscher and P. Weisz, "Quark confinement and the bosonic string," JHEP 0207, 049 (2002) [arXiv:hep-lat/0207003].

[18] G. 't Hooft, "A planar diagram theory for string interactions," Nucl. Phys. B 72, 461 (1974).

[19] J. Polchinski, "Dirichlet-Branes and Ramond-Ramond Charges," Phys. Rev. Lett. 75, 4724 (1995).

[20] D. J. Gross, "Two-dimensional QCD as a string theory," Nucl. Phys. B 400, $161(1993)$.

[21] D. J. Gross and W. Taylor, "Two-dimensional QCD is a string theory," Nucl. Phys. B 400, 181 (1993).

[22] N. Arkani-Hamed, M. Porrati and L. Randall, "Holography and phenomenology," JHEP 0108, 017 (2001).

[23] J. Polchinski and M. J. Strassler, Phys. Rev. Lett. 88, 031601 (2002); JHEP 0305, 012 (2003).

[24] S. J. Brodsky and G. F. de Téramond, "Light-front hadron dynamics and AdS/CFT correspondence," Phys. Lett. B 582, 211 (2004).

[25] T. Sakai and S. Sugimoto, "Low energy hadron physics in holographic QCD," Prog. Theor. Phys. 113, 843 (2005); "More on a holographic dual of QCD," Prog. Theor. Phys. 114, 1083 (2006).

[26] J. Erlich, E. Katz, D. T. Son and M. A. Stephanov, "QCD and a holographic model of hadrons," Phys. Rev. Lett. 95, 261602 (2005).

[27] J. Erlich, G. D. Kribs and I. Low, "Emerging holography," Phys. Rev. D 73, 096001 (2006). 
[28] L. Da Rold and A. Pomarol, "Chiral symmetry breaking from five dimensional spaces," Nucl. Phys. B 721, 79 (2005); "The scalar and pseudoscalar sector in a five-dimensional approach to chiral symmetry breaking," JHEP 0601, 157 (2006).

[29] K. Ghoroku, N. Maru, M. Tachibana and M. Yahiro, "Holographic model for hadrons in deformed AdS(5) background," Phys. Lett. B 633, 602 (2006).

[30] S. J. Brodsky and G. F. de Teramond, Phys. Rev. Lett. 96, 201601 (2006).

[31] D. T. Son and M. A. Stephanov, "QCD and dimensional deconstruction," Phys. Rev. D 69, 065020 (2004).

[32] A. Karch, E. Katz, D. T. Son and M. A. Stephanov, "Linear confinement and AdS/QCD," Phys. Rev. D 74, 015005 (2006).

[33] C. Csaki and M. Reece, "Toward a systematic holographic QCD: A braneless approach," JHEP 0705, 062 (2007).

[34] T. Hambye, B. Hassanain, J. March-Russell and M. Schvellinger, "On the $\operatorname{Delta}(\mathrm{I})=1 / 2$ rule in holographic QCD," Phys. Rev. D 74, 026003 (2006); arXiv:hep-ph/0612010.

[35] A. V. Radyushkin, "Holographic wave functions, meromorphization and counting rules," Phys. Lett. B 642, 459 (2006).

[36] H. R. Grigoryan and A. V. Radyushkin, "Form Factors and Wave Functions of Vector Mesons in Holographic QCD," Phys. Lett. B 650, 421 (2007).

[37] H. R. Grigoryan and A. V. Radyushkin, "Structure of Vector Mesons in Holographic Model with Linear Confinement," Phys. Rev. D 76, 095007 (2007).

[38] H. R. Grigoryan and A. V. Radyushkin, "Pion Form Factor in Chiral Limit of Hard-Wall AdS/QCD Model," Phys. Rev. D 76, 115007 (2007).

[39] H. R. Grigoryan, "Dimension Six Correction to the Vector Sector of AdS/QCD Model," Phys. Lett. B 662, 158 (2008).

[40] H. R. Grigoryan and A. V. Radyushkin, "Anomalous Form Factor of the Neutral Pion in Extended AdS/QCD Model with Chern-Simons Term," Phys. Rev. D 77, 115024 (2008).

[41] J.C.C.A. Kneser, Math. Ann. LXIII, 447 (1907); A.J.W. Sommerfeld, Jahresber. Deutsch. Mat. Ver., XXI, 309 (1912); H. Bateman and A. Erdélyi, Higher Transcendental Functions, vol. 2 (McGraw-Hill Book Co., New York 1953); V. G. Vaccaro and L. Verolino, Nuovo Cim. B 113, 1527 (1998)

[42] R. G. Arnold, C. E. Carlson and F. Gross, Phys. Rev. C 21, 1426 (1980). 
[43] M. S. Bhagwat and P. Maris, "Vector meson form factors and their quark-mass dependence," arXiv:nucl-th/0612069.

[44] B. G. Lasscock, J. Hedditch, D. B. Leinweber and A. G. Williams, "Vector meson electromagnetic form factors," PoS LAT2006, 114 (2006) [arXiv:heplat/0611029].

[45] M. Shifman, "Highly excited hadrons in QCD and beyond," arXiv:hep$\mathrm{ph} / 0507246$.

[46] S. Hong, S. Yoon and M. J. Strassler, "On the couplings of vector mesons in AdS/QCD," JHEP 0604, 003 (2006); arXiv:hep-ph/0501197.

[47] E. Schreiber, "Excited mesons and quantization of string endpoints," arXiv:hep-th/0403226.

[48] S. Eidelman et al. [Particle Data Group], "Review of particle physics," Phys. Lett. B 592, 1 (2004); H. M. Choi and C. R. Ji, "Distribution amplitudes and decay constants for (pi, K, rho, $\mathrm{K}^{*}$ ) mesons in light-front quark model," Phys. Rev. D 75, 034019 (2007).

[49] S. J. Brodsky and G. F. de Teramond, "Light-Front Dynamics and AdS/QCD: The Pion Form Factor in the Space- and Time-Like Regions," arXiv:0707.3859 [hep-ph].

[50] T. Horn et al. [Fpi2 Collaboration], "Determination of the charged pion form factor at $\mathrm{Q}^{* *} 2=1.60-(\mathrm{GeV} / \mathrm{c})^{* *} 2$ and 2.45- $(\mathrm{GeV} / \mathrm{c})^{* *} 2$," Phys. Rev. Lett. 97, 192001 (2006).

[51] H. J. Hippe, "Nambu-Jona-Lasinio model compared with chiral perturbation theory: The Pion radius in SU(2) revisited," Phys. Rev. C 522172 (1995).

[52] M. A. B. Beg and A. Zepeda, "Pion radius and isovector nucleon radii in the limit of small pion mass," Phys. Rev. D 6, 2912 (1972).

[53] M. K. Volkov and V. N. Pervushin, "The Electromagnetic form-factor of the pion," Phys. Lett. B 51, 356 (1974).

[54] S. B. Gerasimov, "Meson Structure Constants In A Model Of The Quark Diagrams," Yad. Fiz. 29, 513 (1979) [Sov. J. Nucl. Phys. 29, 259 (1979 ERRAT,32,156.1980)].

[55] V. Bernard and D. Vautherin, "ELECTROMAGNETIC POLARIZABILITIES OF PSEUDOSCALAR GOLDSTONE BOSONS," Phys. Rev. D 40, 1615 (1989).

[56] A. V. Radyushkin, "Quark-Hadron Duality and Intrinsic Transverse Momentum," Acta Phys. Polon. B 26, 2067 (1995) [arXiv:hep-ph/9511272]. 
[57] J. Hirn, N. Rius and V. Sanz, "Geometric approach to condensates in holographic QCD," Phys. Rev. D 73, 085005 (2006) [arXiv:hep-ph/0512240].

[58] J. Hirn and V. Sanz, "Interpolating between low and high energy QCD via a 5D Yang-Mills model," JHEP 0512, 030 (2005).

[59] H. J. Kwee and R. F. Lebed, "Pion Form Factors in Holographic QCD," JHEP 0801, 027 (2008); "Pion Form Factor in Improved Holographic QCD Backgrounds," arXiv:0712.1811 [hep-ph].

[60] S. J. Brodsky and J. R. Hiller, "Universal Properties Of The Electromagnetic Interactions Of Spin One Systems," Phys. Rev. D 46, 2141 (1992).

[61] H.M. Choi and C.R. Ji, Phys. Rev. D 59, 074015 (1999); Phys. Rev. D 70, 053015 (2004).

[62] C.J. Burden, C.D. Roberts and M.J. Thomson, "Electromagnetic Form Factors of Charged and Neutral Kaons," Phys. Lett. B 371, 163 (1996); F. T. Hawes and M. A. Pichowsky, "Electromagnetic form factors of light vector mesons," Phys. Rev. C 59, 1743 (1999).

[63] J.P.B. de Melo and T. Frederico, "Covariant and light-front approaches to the rho meson electromagnetic form-factors," Phys. Rev. C 55, 2043 (1997).

[64] L. L. Frankfurt, M. Strikman and T. Frederico, "Deuteron form-factors in the light cone quantum mechanics 'good' component approach," Phys. Rev. C 48, 2182 (1993).

[65] P. Maris and P.C. Tandy, "The quark photon vertex and the pion charge radius," Phys. Rev. C 61, 045202 (2000); P. Maris, "Hadron physics and the Dyson-Schwinger equations of QCD," AIP Conf. Proc. 892, 65 (2007).

[66] J. N. Hedditch, W. Kamleh, B. G. Lasscock, D. B. Leinweber, A. G. Williams and J. M. Zanotti, "Pseudoscalar and vector meson form factors from lattice QCD," Phys. Rev. D 75, 094504 (2007).

[67] S. L. Adler, "Axial vector vertex in spinor electrodynamics," Phys. Rev. 177, 2426 (1969); J. S. Bell and R. Jackiw, "A PCAC puzzle: pi0 $\rightarrow$ gamma gamma in the sigma model," Nuovo Cim. A 60, 47 (1969); J. S. Schwinger, "On gauge invariance and vacuum polarization," Phys. Rev. 82, 664 (1951).

[68] J. M. Cornwall, Phys. Rev. Lett. 16, 1174 (1966).

[69] D. J. Gross and S. B. Treiman, "How to see the light cone," Phys. Rev. D 4, 2105 (1971).

[70] S. J. Brodsky, T. Kinoshita and H. Terazawa, "Two Photon Mechanism Of Particle Production By High-Energy Colliding Beams," Phys. Rev. D 4, 1532 (1971). 
[71] J. Parisi and P. Kessler, "Study of the vertices pi0 gamma gamma, eta gamma gamma and eta-prime gamma gamma with almost real photons," Lett. Nuovo Cim. 2, 755 (1971).

[72] M. Jacob and T. T. Wu, "THE DECAY Z $\rightarrow$ pi0 gamma," Phys. Lett. B 232, 529 (1989).

[73] G. P. Lepage and S. J. Brodsky, "Exclusive Processes In Perturbative Quantum Chromodynamics," Phys. Rev. D 22, 2157 (1980).

[74] S. J. Brodsky and G. P. Lepage, "Large Angle Two Photon Exclusive Channels In Quantum Chromodynamics," Phys. Rev. D 24, 1808 (1981).

[75] G. P. Lepage, S. J. Brodsky, T. Huang and P. B. Mackenzie, "Hadronic Wave Functions In QCD", Report CLNS-82-522 (1982), published in Proceedings of 1981 Banff Summer Inst. (1982)

[76] V. L. Chernyak and A. R. Zhitnitsky, "Asymptotic Behavior Of Exclusive Processes In QCD," Phys. Rept. 112, 173 (1984).

[77] A. V. Efremov and A. V. Radyushkin, "Factorization And Asymptotical Behavior Of Pion Form-Factor In QCD," Phys. Lett. B 94, 245 (1980).

[78] A. V. Radyushkin, "Deep elastic processes of composite particles in field theory and asymptotic freedom," JINR-P2-10717 (1977); English translation: arXiv:hep-ph/0410276.

[79] G. P. Lepage and S. J. Brodsky, "Exclusive Processes In Quantum Chromodynamics: Evolution Equations For Hadronic Wave Functions And The FormFactors Of Mesons," Phys. Lett. B 87, 359 (1979).

[80] H. J. Behrend et al. [CELLO Collaboration], "A Measurement of the pi0, eta and eta-prime electromagnetic form-factors," Z. Phys. C 49, 401 (1991).

[81] J. Gronberg et al. [CLEO Collaboration], "Measurements of the meson photon transition form factors of light pseudoscalar mesons at large momentum transfer," Phys. Rev. D 57, 33 (1998)

[82] F. del Aguila and M. K. Chase, "Higher Order QCD Corrections To Exclusive Two Photon Processes," Nucl. Phys. B 193, 517 (1981).

[83] E. Braaten, "QCD Corrections To Meson - Photon Transition Form-Factors," Phys. Rev. D 28, 524 (1983).

[84] E. P. Kadantseva, S. V. Mikhailov and A. V. Radyushkin, "Total Alpha-S Corrections To Processes Gamma* Gamma* $\rightarrow$ Pi0 And Gamma* Pi $\rightarrow$ Pi In A Perturbative QCD," Yad. Fiz. 44, 507 (1986) [Sov. J. Nucl. Phys. 44, 326 (1986)]. 
[85] I. V. Musatov and A. V. Radyushkin, "Transverse momentum and Sudakov effects in exclusive QCD processes: gamma* gamma pi0 form factor," Phys. Rev. D 56, 2713 (1997).

[86] A. Khodjamirian, "Form factors of gamma* rho $\rightarrow$ pi and gamma* gamma $\rightarrow$ pi0 transitions and light-cone sum rules," Eur. Phys. J. C 6, 477 (1999).

[87] A. Schmedding and O. I. Yakovlev, "Perturbative effects in the form factor gamma gamma* $\rightarrow$ pi0 and extraction of the pion wave function from CLEO data," Phys. Rev. D 62, 116002 (2000).

[88] A. P. Bakulev, S. V. Mikhailov, A. V. Pimikov and N. G. Stefanis, "Pion structure in QCD: From theory to lattice to experimental data," arXiv:0710.2275 [hep-ph].

[89] M. B. Voloshin, "QCD Calculation Of Pi0 Gamma Gamma Vertex At Equal Euclidean $\mathrm{Q}^{* *} 2$ Of Both Photons," Preprint ITEP-8-1982.

[90] V. A. Nesterenko and A. V. Radyushkin, "Comparison Of The QCD Sum Rule Approach And Perturbative QCD Analysis For Gamma* Gamma* $\rightarrow$ Pi0 Process," Sov. J. Nucl. Phys. 38, 284 (1983) [Yad. Fiz. 38, 476 (1983)].

[91] S. V. Mikhailov and A. V. Radyushkin, "Quark Condensate Nonlocality and Pion Wave Function in QCD: General Formalism," Sov. J. Nucl. Phys. 49, 494 (1989) [Yad. Fiz. 49, 794 (1988)].

[92] H. Ito, W. W. Buck and F. Gross, "Electromagnetic Properties Of The Pion As A Composite Nambu-Goldstone Boson," Phys. Rev. C 45, 1918 (1992).

[93] I. V. Anikin, M. A. Ivanov, N. B. Kulimanova and V. E. Lyubovitskij, "The Extended Nambu-Jona-Lasinio model with separable interaction: Low-energy pion physics and pion nucleon form-factor," Z. Phys. C 65, 681 (1995).

[94] A. V. Radyushkin and R. Ruskov, "Transition Form Factor gamma gamma* $\rightarrow$ pi0 and QCD sum rules," Nucl. Phys. B 481, 625 (1996).

[95] P. Kroll and M. Raulfs, "The pi gamma transition form factor and the pion wave function," Phys. Lett. B 387, 848 (1996); R. Jakob, P. Kroll and M. Raulfs, "Meson - Photon Transition Form-Factors," J. Phys. G 22, 45 (1996).

[96] V. V. Anisovich, D. I. Melikhov and V. A. Nikonov, "Photon meson transition form factors gamma pi0, gamma eta and gamma eta' at low and moderately high Q**2," Phys. Rev. D 55, 2918 (1997).

[97] D. Kekez and D. Klabucar, "gamma* gamma $\rightarrow$ pi0 transition and asymptotics of gamma* gamma and gamma* gamma* transitions of other unflavored pseudoscalar mesons," Phys. Lett. B 457, 359 (1999). 
[98] I. V. Anikin, A. E. Dorokhov and L. Tomio, "Pion structure in the instanton liquid model," Phys. Part. Nucl. 31, 509 (2000) [Fiz. Elem. Chast. Atom. Yadra 31, 1023 (2000)].

[99] P. Maris and P. C. Tandy, "Electromagnetic transition form factors of light mesons," Phys. Rev. C 65, 045211 (2002)

[100] A. E. Dorokhov, M. K. Volkov and V. L. Yudichev, "Transition form factors and light cone distribution amplitudes of pseudoscalar mesons in the chiral quark model," Phys. Atom. Nucl. 66, 941 (2003) [Yad. Fiz. 66, 973 (2003)].

[101] B. W. Xiao and B. Q. Ma, "Photon meson transition form factors of light pseudoscalar mesons," Phys. Rev. D 71, 014034 (2005).

[102] E. Ruiz Arriola and W. Broniowski, "Pion transition form factor and distribution amplitudes in large-N(c) Regge model," Phys. Rev. D 74, 034008 (2006).

[103] H. Boschi-Filho and N. R. F. Braga, "Gauge / string duality and scalar glueball mass ratios," JHEP 0305, 009 (2003); "QCD / string holographic mapping and glueball mass spectrum," Eur. Phys. J. C 32, 529 (2004).

[104] N. Evans, A. Tedder and T. Waterson, "Improving the infra-red of holographic descriptions of QCD," JHEP 0701, 058 (2007).

[105] R. Casero, E. Kiritsis and A. Paredes, "Chiral symmetry breaking as open string tachyon condensation," Nucl. Phys. B 787, 98 (2007).

[106] U. Gursoy and E. Kiritsis, "Exploring improved holographic theories for QCD: Part I," JHEP 0802, 032 (2008).

[107] U. Gursoy, E. Kiritsis and F. Nitti, "Exploring improved holographic theories for QCD: Part II," JHEP 0802, 019 (2008).

[108] O. Bergman, S. Seki and J. Sonnenschein, "Quark mass and condensate in HQCD," JHEP 0712, 037 (2007).

[109] J. Erdmenger, K. Ghoroku and I. Kirsch, "Holographic heavy-light mesons from non-Abelian DBI," JHEP 0709, 111 (2007).

[110] J. Erdmenger, N. Evans, I. Kirsch and E. Threlfall, "Mesons in Gauge/Gravity Duals - A Review," arXiv:0711.4467 [hep-th].

[111] A. Dhar and P. Nag, "Sakai-Sugimoto model, Tachyon Condensation and Chiral symmetry Breaking," JHEP 0801, 055 (2008).

[112] Z. Abidin and C. E. Carlson, "Gravitational Form Factors of Vector Mesons in an AdS/QCD Model," Phys. Rev. D 77, 095007 (2008); "Gravitational Form Factors in the Axial Sector from an AdS/QCD Model," arXiv:0804.0214 [hepph]. 
[113] C. T. Hill, "Exact equivalence of the D = 4 gauged Wess-Zumino-Witten term and the D = 5 Yang-Mills Chern-Simons term," Phys. Rev. D 73, 126009 (2006).

[114] S. K. Domokos and J. A. Harvey, "Baryon number-induced Chern-Simons couplings of vector and axial-vector mesons in holographic QCD," Phys. Rev. Lett. 99, 141602 (2007).

[115] S. S. Chern and J. Simons, "Characteristic Forms And Geometric Invariants," Annals Math. 99, 48 (1974).

[116] T. Fujiwara, T. Kugo, H. Terao, S. Uehara and K. Yamawaki, "Nonabelian Anomaly And Vector Mesons As Dynamical Gauge Bosons Of Hidden Local Symmetries," Prog. Theor. Phys. 73, 926 (1985).

[117] J. Wess and B. Zumino, "Consequences of anomalous Ward identities," Phys. Lett. B 37, 95 (1971).

[118] E. Witten, "Global Aspects Of Current Algebra," Nucl. Phys. B 223, 422 (1983).

[119] U. G. Meissner, "Low-Energy Hadron Physics From Effective Chiral Lagrangians With Vector Mesons," Phys. Rept. 161, 213 (1988).

[120] F. Farzanpay et al., "Measurement of the slope of the pi0 electromagnetic form-factor," Phys. Lett. B 278, 413 (1992).

[121] R. Meijer Drees et al. [SINDRUM-I Collaboration], "Measurement of the pi0 electromagnetic transition form-factor," Phys. Rev. D 45, 1439 (1992).

[122] H. Fonvieille et al., "DALITZ DECAY: PI0 $\rightarrow$ GAMMA E+ E- AND THE PIO ELECTROMAGNETIC TRANSITION FORM-FACTOR," Phys. Lett. B 233, 65 (1989).

[123] A. Gasparian, et al. "A Precision Measurement of the Neutral Pion Lifetime via the Primakoff Effect", JLab proposal PR-02-103, http://www.jlab.org/primex/

[124] V. A. Nesterenko and A. V. Radyushkin, "Sum Rules And Pion Form-Factor In QCD," Phys. Lett. B 115, 410 (1982).

[125] E. Katz and M. D. Schwartz, "An Eta Primer: Solving the U(1) Problem with AdS/QCD," JHEP 0708, 077 (2007). 


\section{Appendix: Permission Letters From Journals}

Supplier

Registered Company Number

Customer name

License Number

License date

Licensed content publisher

Licensed content publication

Licensed content title

Licensed content author

Licensed content date

Volume number

Issue number

Pages

Type of Use

Portion

Format

You are an author of the Elsevier article

Expected publication date

Elsevier VAT number
Elsevier Limited

The Boulevard, Langford Lane Kidlington,Oxford,OX5 1GB,UK

1982084

Hovhannes R Grigoryan

1958350373547

May 29, 2008

Elsevier Limited

Physics Letters B

Form factors and wave functions of vector mesons in holographic QCD

Hovhannes R. Grigoryan and Anatoly V. Radyushkin

12 July 2007

650

5-6

7

Thesis / Dissertation

Full article

Both print and electronic

Yes

Sep 2008

GB 494627212 
Supplier

Registered Company Number

Customer name

License Number

License date

Licensed content publisher

Licensed content publication

Licensed content title

Licensed content author

Licensed content date

Volume number

Issue number

Pages

Type of Use

Portion

Format

You are an author of the Elsevier article

Expected publication date

Elsevier VAT number
Elsevier Limited

The Boulevard,Langford Lane

Kidlington,Oxford,OX5 1GB,UK

1982084

Hovhannes R Grigoryan

1958350141594

May 29, 2008

Elsevier Limited

Physics Letters B

Dimension six corrections to the vector sector of AdS/QCD model

Hovhannes R. Grigoryan

17 April 2008

662

2

Thesis / Dissertation

Full article

Both print and electronic

Yes

Sep 2008

GB 494627212 


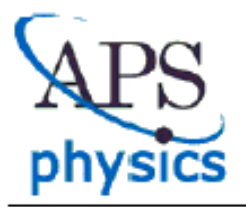

AMERICAN PHYSICAL SOCIETY

One Physics Ellipse, College Park, MD $20740 \cdot \underline{h t t p: / w w w . a p s . o n z ~}$

May 30,2008

Hovhannes Grigoryan

hgrigo1@jlab.org

$\underline{\text { Ref \# } 6316}$

Thank you for your permission request dated May 29, 2008. We are pleased to grant you a non-exclusive, nontransferable permission, English rights, limited to print and World Wide Web format only, provided you meet the criteria outlined below. Permission is for a one-time use and does not include permission for future editions, updates, additional electronic forms, databases, translations, or any other matters. Permission must be sought for each additional use. This permission does not include the right to modify APS material.

Please print the required copyright credit line on the first page that the material appears: "Reprinted (abstract/excerpt/figure) with permission from [FULL REFERENCE CITATION] as follows: authors names, journal title, volume number, page number and year of publication. Copyright (YEAR) by the American Physical Society.

The following language must appear somewhere on the website: "Readers may view, browse, and/or download material for temporary copying purposes only, provided these uses are for noncommercial personal purposes. Except as provided by law, this material may not be further reproduced, distributed, transmitted, modified, adapted, performed, displayed, published, or sold in whole or part, without prior written permission from the American Physical Society."

Provide a hyperlink from the reprinted APS material (the hyperlink may be embedded in the copyright credit line). APS's link manager technology makes it convenient and easy to provide links to individual articles in APS journals. For information, see: $\underline{\text { http://publish. aps.org/linkfaq.html }}$

You must also obtain permission from at least one of the authors for each separate work, if you haven't done so already. The author's name and address can be found on the first page of the published Article.

Use of the APS material must not imply any endorsement by the American Physical Society.

Permission is granted for use of the following APS material only:

- Phys. Rev. D Vol. 76, 095007 (2007)

- Phys. Rev. D Vol. 76, 115007 (2007)

- Phys. Rev. D - Manuscript es2008mar13_099 (accepted for publication)

Permission is limited to the single title specified or single edition of the publication as follows:

- Ph.D. Thesis by Hovhannes Grigoryan

If you have any questions, please refer to the Copyright FAQ at: http://forms.aps.org/author/copyfaq.html or contact me at assocpub@aps.org.

Sincerely,

Silem Lamauca

Eileen LaManca

Publications Marketing Coordinator 


\section{Vita}

Hovhannes Roman Grigoryan was born on October 24, 1980, in Yerevan, Armenia. He graduated from the high-school No.132 in Yerevan, with honors in May of 1997 and entered the Department of Physics of Yerevan State University in September of 1997, where he received his Bachelor of Science degree with honors in theoretical physics in June of 2001 and was awarded Master of Science degree in theoretical physics in June of 2003. He was admitted to the doctoral program of the Department of Physics and Astronomy of Louisiana State University in September of 2001, where his degree of Doctor of Philosophy in physics is expected to be awarded in August of 2008. 